\title{
Ausschreibungen zur Honorierung von Umweltleistungen
}

\author{
Eine praxisbezogene Analyse \\ am Beispiel ökologischer Leistungen der Landwirtschaft
}

\author{
Dissertation \\ zur Erlangung des Doktorgrades \\ der Fakultät für Agrarwissenschaften \\ der Georg-August-Universität Göttingen
}

\author{
vorgelegt von \\ Markus Groth \\ geboren in Hildesheim
}

Göttingen, September 2006 
D 7

1. Referent: $\quad$ Prof. Dr. Rainer Marggraf (Universität Göttingen)

2. Korreferentin: $\quad$ Prof. Dr. Karin Holm-Müller (Universität Bonn)

Tag der mündlichen Prüfung: 16. November 2006 


\section{Inhaltsverzeichnis}

Abbildungsverzeichnis............................................................................................... VI

Tabellenverzeichnis.....................................................................................................VIII

Abkürzungsverzeichnis ......................................................................................... XII

1 Einleitung und Aufbau ............................................................................ 1

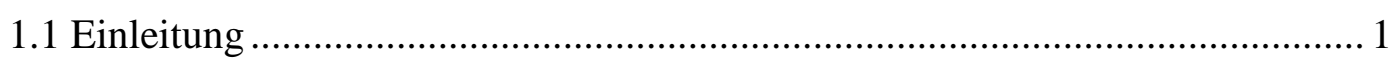

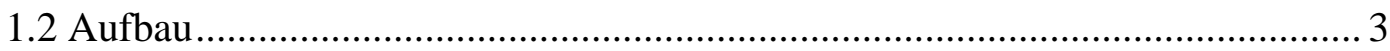

2 Agrarumweltpolitischer Rahmen ...............................................................6

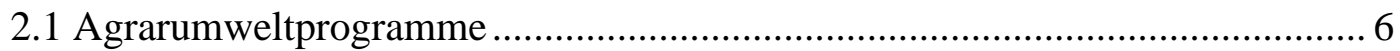

2.2 Kritikpunkte derzeitiger Agrarumweltprogramme ........................................ 8

2.3 Ansätze zur Weiterentwicklung der Agrarumweltpolitik .............................. 10

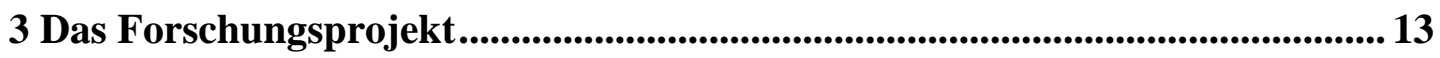

3.1 Komponenten des Honorierungssystems …................................................... 14

3.1.1 Ergebnisorientierung - Die ökologischen Güter Grünland....................... 14

3.1.2 Subsidiarität - Der Regionale Beirat Northeim ..................................... 16

3.1.3 Partizipation - Die regionalen Bevölkerungspräferenzen........................ 17

3.1.4 Effizienz - Das Ausschreibungsverfahren .............................................. 18

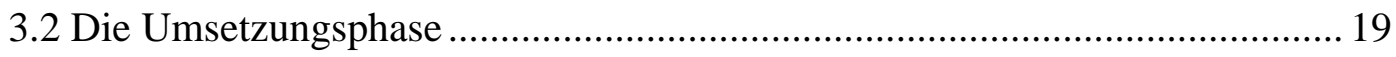

3.2.1 Die Modellregion - Der Landkreis Northeim........................................ 19

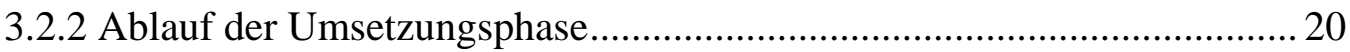

4 Transaktionskostenökonomik...................................................................... 23

4.1 Exkurs: Entwicklung der Transaktionskostenökonomik ................................ 25

4.2 Grundlegende Definitionen und begriffliche Abgrenzungen........................... 33

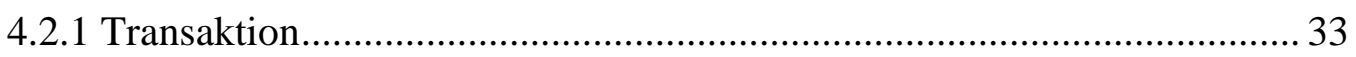

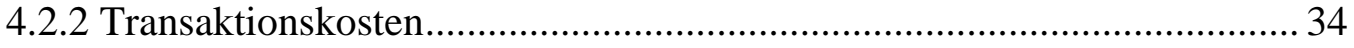

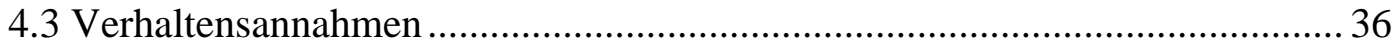

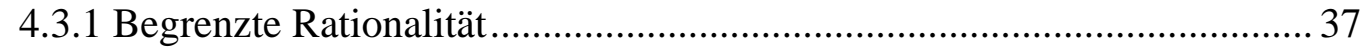

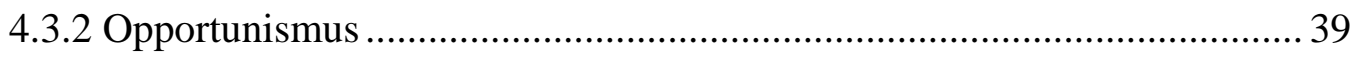

4.4 Dimensionen von Transaktionen ................................................................ 40 


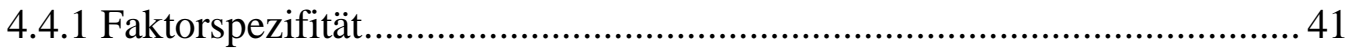

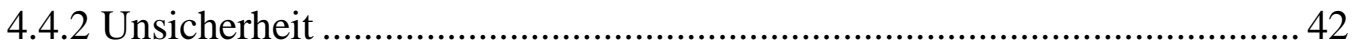

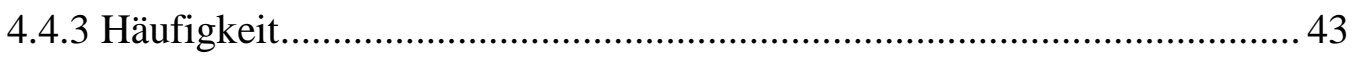

4.4.4 Exkurs: Die fundamentale Transformation.......................................... 44

4.5 Beherrschung und Überwachung von Transaktionen ..................................... 45

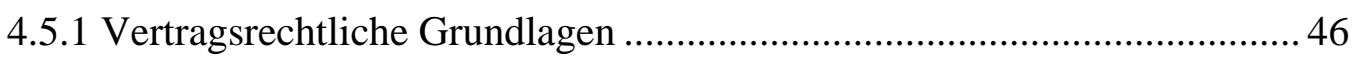

4.5.2 Beherrschungs- und Überwachungsstrukturen ...................................... 47

4.6 Abgrenzungen von Transaktionskosten ....................................................... 49

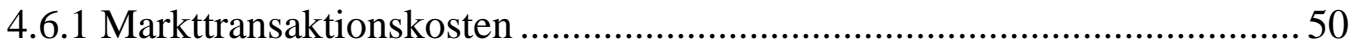

4.6.2 Unternehmenstransaktionskosten.......................................................... 52

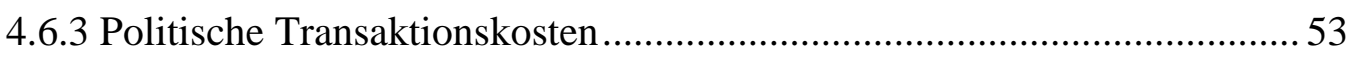

4.7 Kritik am Ansatz der Transaktionskostenökonomik..................................... 54

5 Ausschreibungen .........................................................................................5 58

5.1 Auktionstheoretische Grundlagen .................................................................. 59

5.1.1 Gegenstand und Ziele von Auktionen.................................................. 61

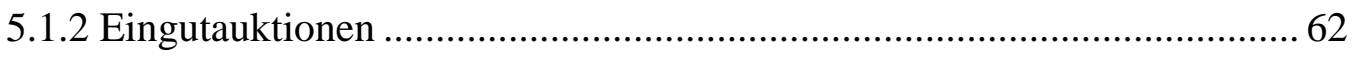

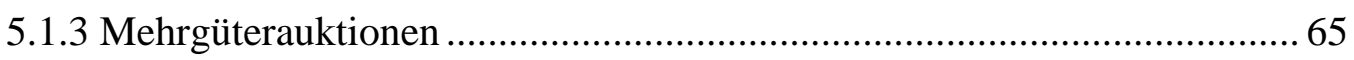

5.1.4 Auktionsmodelle und Äquivalenzeigenschaften.....................................6 68

5.2 Ausschreibungen für Umweltleistungen ..................................................... 71

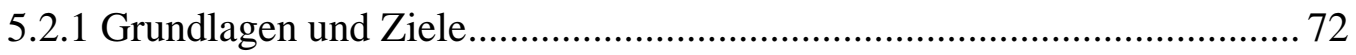

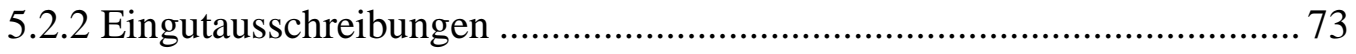

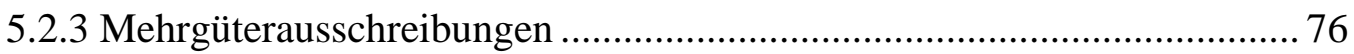

5.2.4 Weitere Ausgestaltungsmöglichkeiten................................................. 81

5.2.5 Ausschreibungsdesign im Rahmen des Forschungsprojekts .................... 84

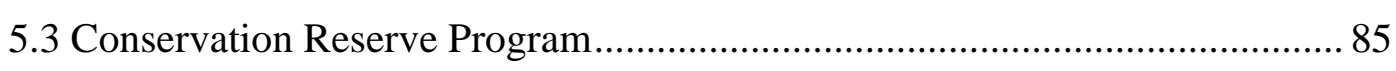

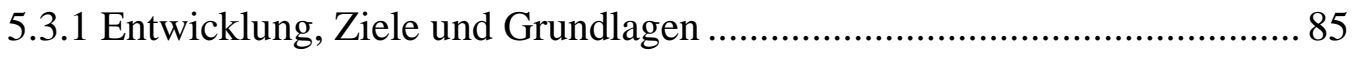

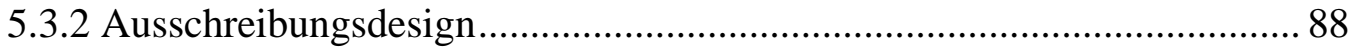

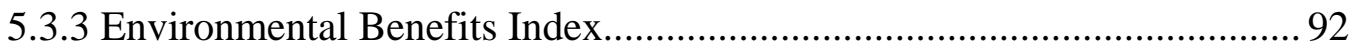

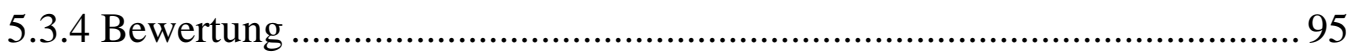

5.4 Weitere Konzepte des Einsatzes von Ausschreibungen................................. 96

5.4.1 BushTender Trial in Australien............................................................... 97

5.4.2 Modellausschreibungen in Nordrhein-Westfalen .................................. 99 
6 Die transaktionskostenökonomische Analyse................................................. 102

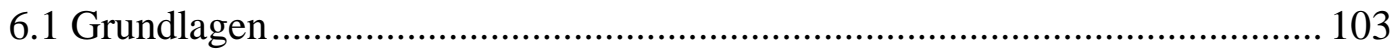

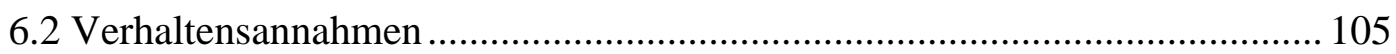

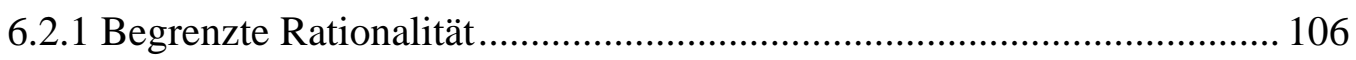

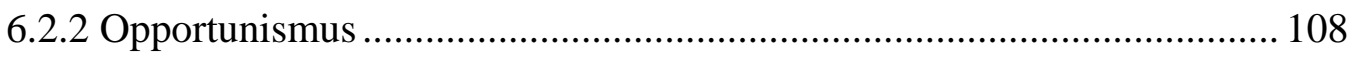

6.3 Dimensionen von Transaktionen ............................................................... 112

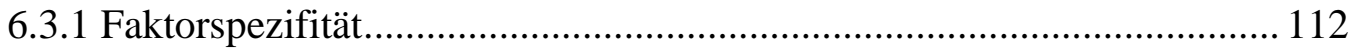

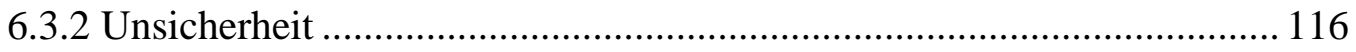

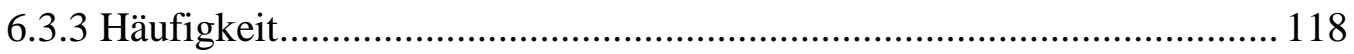

6.4 Beherrschung und Überwachung von Transaktionen .................................. 119

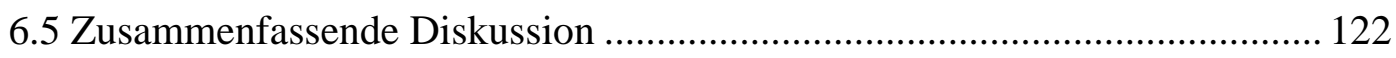

7 Die Umsetzung und Ergebnisse der Ausschreibungen ..................................... 127

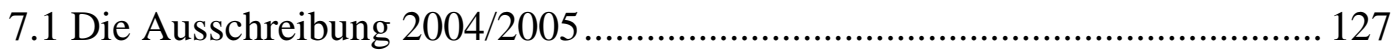

7.1.1 Grundlagen, zeitlicher Ablauf und Beiratsentscheidung ........................ 128

7.1.2 Ergebnisse - Ökologisches Gut Grünland I........................................ 130

7.1.2.1 Eingegangene Angebote............................................................. 131

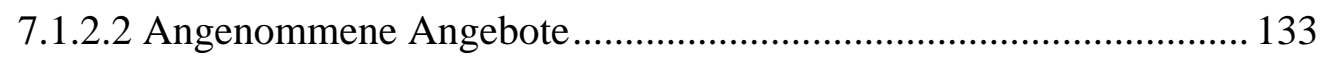

7.1.2.3 Auszahlung nach der Kontrolle................................................... 135

7.1.3 Ergebnisse - Ökologisches Gut Grünland II....................................... 137

7.1.3.1 Eingegangene und angenommene Angebote ................................ 137

7.1.3.2 Auszahlung nach der Kontrolle..................................................... 139

7.1.4 Ergebnisse - Ökologisches Gut Grünland III ...................................... 141

7.1.4.1 Eingegangene und angenommene Angebote ................................ 141

7.1.4.2 Auszahlung nach der Kontrolle................................................... 143

7.1.5 Zusammenfassende Ergebnisse der Angebotspreise............................. 144

7.1.5.1 Eingegangene Angebote................................................................ 144

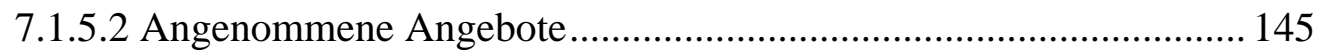

7.1.5.3 Auszahlung nach der Kontrolle..................................................... 146

7.1.6 Preiskalkulation und Bieterverhalten .................................................... 147

7.1.6.1 Befragungsdesign und allgemeine Daten..................................... 148

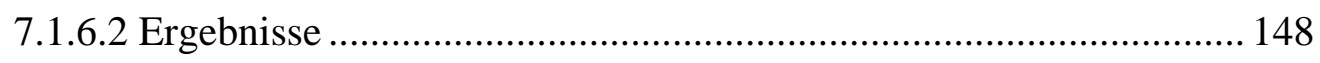

7.1.6.3 Zusammenfassende Betrachtung................................................ 156

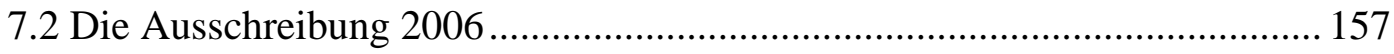


7.2.1 Grundlagen, zeitlicher Ablauf und Beiratsentscheidung ....................... 158

7.2.2 Ergebnisse - Ökologisches Gut Grünland I .......................................... 160

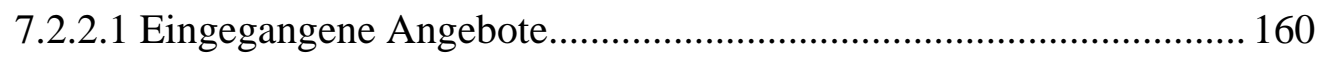

7.2.2.2 Angenommene Angebote........................................................ 162

7.2.3 Ergebnisse - Ökologisches Gut Grünland II........................................ 163

7.2.3.1 Eingegangene Angebote............................................................ 164

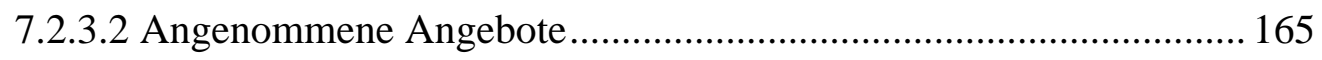

7.2.4 Ergebnisse - Ökologisches Gut Grünland III ....................................... 167

7.2.5 Zusammenfassende Ergebnisse der Angebotspreise.............................. 169

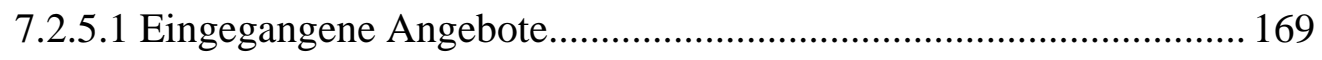

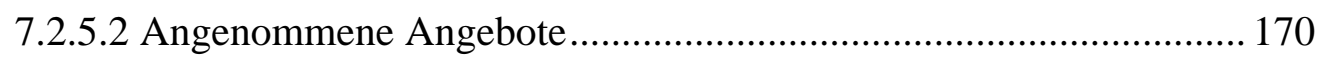

7.2.6 Preiskalkulation und Bieterverhalten der Landwirte.............................. 171

7.2.6.1 Befragungsdesign und allgemeine Daten..................................... 171

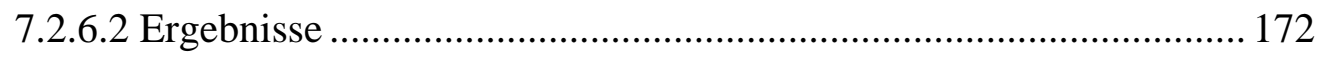

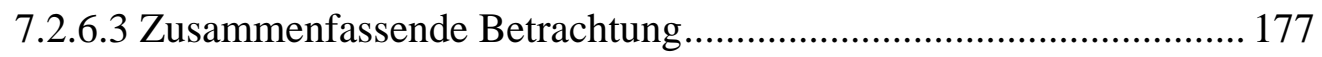

7.3 Zusammenfassende Diskussion ................................................................ 177

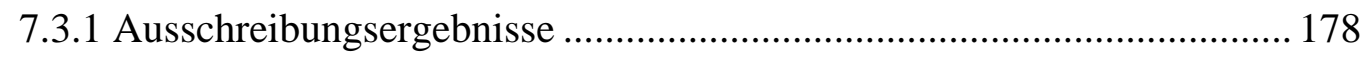

7.3.1.1 Eingegangene Angebote........................................................... 178

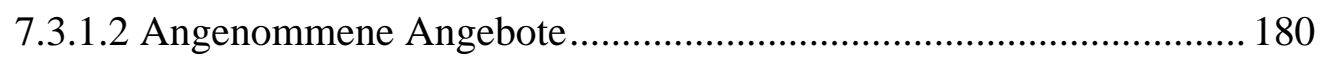

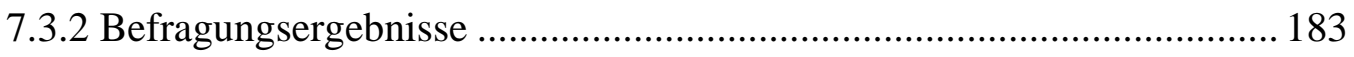

8 Die Ermittlung und Bewertung der Transaktionskosten ................................ 187

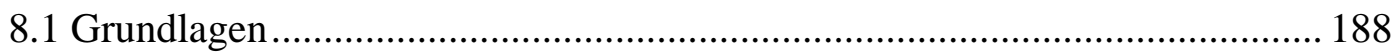

8.2 Definition und methodisches Vorgehen....................................................... 191

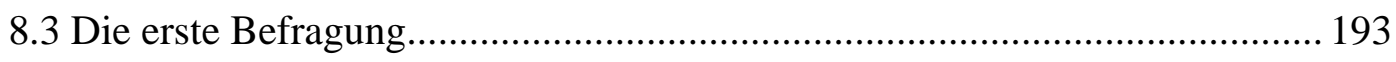

8.3.1 Konzeption und teilnehmende Landwirte ............................................. 193

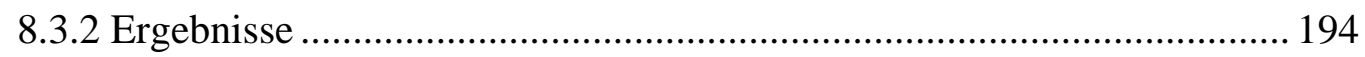

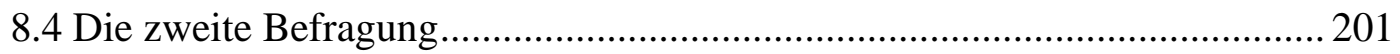

8.4.1 Konzeption und teilnehmende Landwirte .............................................. 201

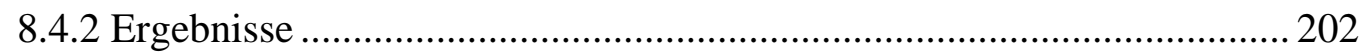

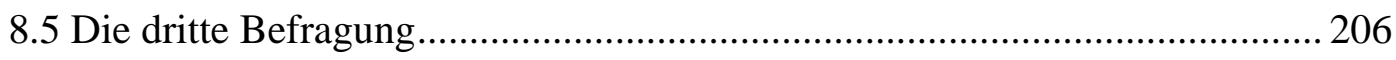

8.5.1 Konzeption und teilnehmende Landwirte ............................................ 206

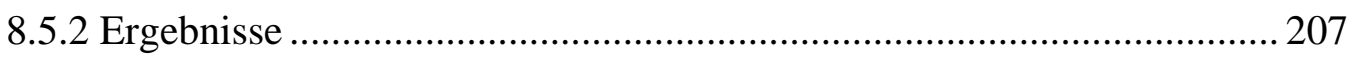

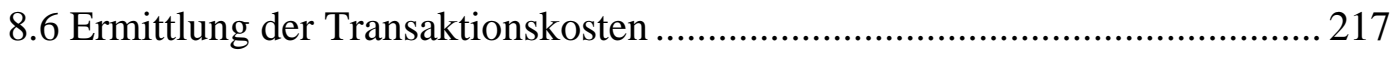


8.6.1 Transaktionskosten der Ausschreibung 2004/2005 ............................... 218

8.6.2 Transaktionskosten der Ausschreibung 2006....................................... 220

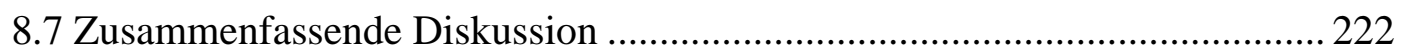

9 Weiterer Forschungsbedarf .................................................................................. 228

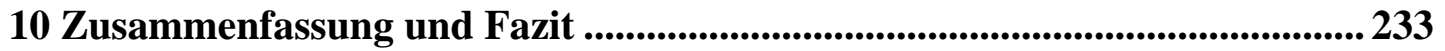

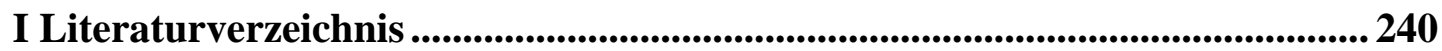

II Verzeichnis der Gesetzesgrundlagen..................................................................... 259

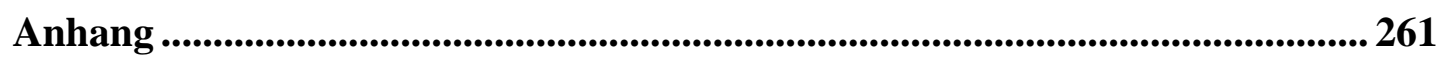




\section{Abbildungsverzeichnis}

Abb. 3-1: Struktur des Honorierungssystems in der Umsetzungsphase.

Abb. 4-1: Beherrschungs- und Überwachungsstrukturen von

Transaktionsbeziehungen

Abb. 7-1: Angebotspreise in $€$ pro Hektar der eingegangenen Angebote für das ökologische Gut Grünland I und Häufigkeit ihres Auftretens (2004/2005)

Abb. 7-2: Angebotspreise in $€$ pro Hektar der angenommenen Angebote für das ökologische Gut Grünland I und Häufigkeit ihres Auftretens (2004/2005)

Abb. 7-3: Angebotspreise in $€$ pro Hektar der erfüllten Angebote für das ökologische Gut Grünland I und Häufigkeit ihres Auftretens (2004/2005)

Abb. 7-4: Angebotspreise in $€$ pro Hektar der eingegangenen und angenommenen Angebote für das ökologische Gut Grünland II und Häufigkeit ihres Auftretens (2004/2005) 138

Abb. 7-5: Angebotspreise in € pro Hektar der erfüllten Angebote für das ökologische Gut Grünland II und Häufigkeit ihres Auftretens (2004/2005) 140

Abb. 7-6: Angebotspreise in $€$ pro Hektar der eingegangenen und angenommenen Angebote für das ökologische Gut Grünland III und Häufigkeit ihres Auftretens (2004/2005)

Abb. 7-7: Angebotspreise in $€$ pro Hektar der erfüllten Angebote für das ökologische Gut Grünland III und Häufigkeit ihres Auftretens $(2004 / 2005)$

Abb. 7-8: Angebotspreise in € pro Hektar der eingegangenen Angebote für das ökologische Gut Grünland I und Häufigkeit ihres Auftretens (2006)... 161

Abb. 7-9: Angebotspreise in $€$ pro Hektar der angenommenen Angebote für das ökologische Gut Grünland I und Häufigkeit ihres Auftretens (2006)... 162

Abb. 7-10: Angebotspreise in € pro Hektar der eingegangenen Angebote für das ökologische Gut Grünland II und Häufigkeit ihres Auftretens (2006) . 164

Abb. 7-11: Angebotspreise in $€$ pro Hektar der angenommenen Angebote für das ökologische Gut Grünland II und Häufigkeit ihres Auftretens (2006) . 166 
Abb. 7-12: Angebotspreise in $€$ pro Hektar der eingegangenen und angenommenen Angebote für das ökologische Gut Grünland III und Häufigkeit ihres Auftretens (2006) 168

Abb. 8-1: Zeitaufwand zur Beschaffung der Ausschreibungsunterlagen in Minuten und Häufigkeit der Nennung (2004/2005). 195

Abb. 8-2: Zeitaufwand zum Lesen und Verstehen der Ausschreibungsunterlagen in Minuten und Häufigkeit der Nennung (2004/2005). 196

Abb. 8-3: Zeitaufwand zur Angebotsermittlung in Minuten und Häufigkeit der Nennung (2004/2005)

Abb. 8-4: Zeitaufwand zum Ausfüllen der Ausschreibungsunterlagen in Minuten und Häufigkeit der Nennung (2004/2005) .

Abb. 8-5: Als angemessen eingeschätzter Stundenlohn für die Angebotserstellung in € und Häufigkeit der Nennung (2004/2005)

Abb. 8-6: Zeitaufwand zum Lesen und Verstehen der Ausschreibungsunterlagen in Minuten und Häufigkeit der Nennung (2006). 208

Abb. 8-7: Zeitaufwand zur Angebotsermittlung in Minuten und Häufigkeit der Nennung (2006)

Abb. 8-8: Zeitaufwand zum Ausfüllen der Ausschreibungsunterlagen in Minuten und Häufigkeit der Nennung (2006)

Abb. 8-9: Als angemessen eingeschätzter Stundenlohn für die Angebotserstellung in $€$ und Häufigkeit der Nennung (2006).... 


\section{Tabellenverzeichnis}

Tab. 5-1: Übersicht über die 1997 teilnahmeberechtigten Flächen im CRP .......... 89

Tab. 5-2: Zusammensetzung des EBI in der 26. Ausschreibungsrunde .................. 95

Tab. 5-3: Ergebnisse der 26. Ausschreibungsrunde im CRP.................................. 96

Tab. 5-4: Ergebnisse der ersten Ausschreibungsrunde - BushTender Trial........... 98

Tab. 5-5: Angenommene Angebote der ersten und zweiten Modellausschreibung in Nordrhein-Westfalen.

Tab. 7-1: Ergebnisse der eingegangenen Angebote für das ökologische Gut

Grünland I (2004/2005).

Tab. 7-2: Ergebnisse der angenommenen Angebote für das ökologische Gut Grünland I (2004/2005).

Tab. 7-3: Ergebnisse der erfüllten Angebote für das ökologische Gut Grünland I (2004/2005)

Tab. 7-4: Ergebnisse der eingegangenen und angenommenen Angebote für das ökologische Gut Grünland II (2004/2005).

Tab. 7-5: Ergebnisse der erfüllten Angebote für das ökologische Gut Grünland II (2004/2005)

Tab. 7-6: Ergebnisse der eingegangenen und angenommenen Angebote für das ökologische Gut Grünland III (2004/2005)

Tab. 7-7: Ergebnisse der erfüllten Angebote für das ökologische Gut Grünland III (2004/2005)

Tab. 7-8: Ergebnisse der eingegangenen Angebote für die ökologischen Güter Grünland I, II und III (2004/2005)....

Tab. 7-9: Ergebnisse der angenommenen Angebote für die ökologischen Güter Grünland I, II und III (2004/2005) 146

Tab. 7-10: Ergebnisse der erfüllten Angebote für die ökologischen Güter Grünland I, II und III (2004/2005).

Tab. 7-11: Ausschreibungsspezifische Kommunikation der Bieter - Differenziert nach Bewirtschaftungs- und Betriebsform (2004/2005)

Tab. 7-12: Einschätzung zukünftiger Preisabsprachen der Bieter - Differenziert nach Art der Bewirtschaftung und Betriebsform (2004/2005). 150 
Tab. 7-13: Berücksichtigung des Zeitaufwands der Angebotsabgabe bei der Preiskalkulation - Differenziert nach Bewirtschaftungs- und Betriebsform (2004/2005)

Tab. 7-14: Berücksichtigung eines Gewinnzuschlags bei der Preiskalkulation Differenziert nach Bewirtschaftungs- und Betriebsform (2004/2005) . 153

Tab. 7-15: Angaben zur Höhe des seitens der Landwirte berücksichtigten Gewinnzuschlags (2004/2005).

Tab. 7-16: Zukünftige Berücksichtigung anderer Angebotspreise - Differenziert nach Bewirtschaftungs- und Betriebsform (2004/2005)

Tab. 7-17: Vermutliche Höhe zukünftiger Angebotspreise - Differenziert nach Bewirtschaftungs- und Betriebsform

Tab. 7-18: Ergebnisse der eingegangenen Angebote für die ökologischen Güter Grünland I (2006).

Tab. 7-19: Ergebnisse der angenommenen Angebote für die ökologischen Güter Grünland I (2006)

Tab. 7-20: Ergebnisse der eingegangenen Angebote für die ökologischen Güter Grünland II (2006)

Tab. 7-21: Ergebnisse der angenommenen Angebote für die ökologischen Güter Grünland II (2006)

Tab. 7-22: Ergebnisse der eingegangenen und angenommenen Angebote für die ökologischen Güter Grünland III (2006).....

Tab. 7-23: Ergebnisse der eingegangenen Angebote für die ökologischen Güter Grünland I, II und III (2006)

Tab. 7-24: Ergebnisse der angenommenen Angebote für die ökologischen Güter Grünland I, II und III (2006).

Tab. 7-25: Ausschreibungsspezifische Kommunikation der Bieter - Differenziert nach Bewirtschaftungs- und Betriebsform (2006)

Tab. 7-26: Einschätzung zukünftiger Preisabsprachen - Differenziert nach Art der Bewirtschaftung und Betriebsform (2006).

Tab. 7-27: Berücksichtigung des Zeitaufwands der Angebotsabgabe bei der Preiskalkulation - Differenziert nach Bewirtschaftungs- und Betriebsform (2006).

Tab. 7-28: Berücksichtigung eines Gewinnzuschlags bei der Preiskalkulation Differenziert nach Bewirtschaftungs- und Betriebsform (2006) 
Tab. 7-29: Angaben zur Höhe des seitens der Landwirte berücksichtigten Gewinnzuschlags (2006) 176

Tab. 7-30: Gegenüberstellung zentraler Ergebnisse der eingegangenen Angebote für die Ausschreibungen 2004/2005 und 2006

Tab. 7-31: Gegenüberstellung zentraler Ergebnisse der angenommenen Angebote für die Ausschreibungen 2004/2005 und 2006 181

Tab. 8-1: Zeitaufwand - Beschaffung der Ausschreibungsunterlagen (2004/2005)

Tab. 8-2: Zeitaufwand - Lesen und Verstehen der Ausschreibungsunterlagen (2004/2005)

Tab. 8-3: Zeitaufwand - Angebotsermittlung (2004/2005)

Tab. 8-4: Zeitaufwand - Ausfüllen der Ausschreibungsunterlagen (2004/2005). 198

Tab. 8-5: Vergleich des Zeitaufwands der Angebotsabgabe mit bisherigen Antragstellungen in Agrarumweltprogrammen (2004/2005). 200

Tab. 8-6: Verfügbarkeit eines Internet-Zugangs und Bereitschaft zur OnlineAntragstellung (2004/2005)

Tab. 8-7: Als angemessen eingeschätzter Stundenlohn für die Angebotsabgabe (2004/2005)

Tab. 8-8: Berücksichtigung des Zeitaufwands der Angebotsabgabe bei der Preiskalkulation - Differenziert nach Bewirtschaftungs- und Betriebsform (2004/2005) 204

Tab. 8-9: Vergleich des vermutlichen Zeitaufwands der Teilnahme an der kommenden Ausschreibung im Vergleich zu der Ausschreibung $2004 / 2005$

Tab. 8-10: Zeitaufwand - Lesen und Verstehen der Ausschreibungsunterlagen (2006) 208

Tab. 8-11: Zeitaufwand - Angebotsermittlung (2006) 209

Tab. 8-12: Zeitaufwand - Ausfüllen der Ausschreibungsunterlagen (2006)

Tab. 8-13: Als angemessen eingeschätzter Stundenlohn für die Angebotsabgabe (2006)

Tab. 8-14: Berücksichtigung des Zeitaufwands der Angebotsabgabe bei der Preiskalkulation - Differenziert nach Bewirtschaftungs- und Betriebsform (2006) 
Tab. 8-15: Vergleich des Zeitaufwands der Angebotsabgabe mit bisherigen Antragstellungen in Agrarumweltprogrammen (2006)..

Tab. 8-16: Verfügbarkeit eines Internet-Zugangs und Bereitschaft zur OnlineAntragstellung (2006)

Tab. 8-17: Mittelwert und Median des Zeitaufwands der Ausschreibung 2004/2005

Tab. 8-18: Mittelwert und Median des als angemessen eingeschätzten Stundenlohns für die Angebotsabgabe (2004/2005) 219

Tab. 8-19: Mittelwert und Median des Zeitaufwands der Ausschreibung 2006..... 220

Tab. 8-20: Mittelwert und Median des als angemessen eingeschätzten Stundenlohns für die Angebotsabgabe 2006.

Tab. 8-21: Gegenüberstellung der Transaktionskosten für die Ausschreibungen 2004/2005 und 2006 auf Grundlage der Mittelwerte

Tab. 8-22: Gegenüberstellung der Transaktionskosten für die Ausschreibungen 2004/2005 und 2006 auf Grundlage der Mediane.

Tab. 8-23: Anteile der Transaktionskosten je Schlag an den Angebotspreisen der eingegangenen Angebote für die Ausschreibungen 2004/2005 und 2006 auf Grundlage der Mittelwerte und der Mediane. 


\section{Abkürzungsverzeichnis}

\begin{tabular}{|c|c|}
\hline Abs. & Absatz \\
\hline $\mathrm{BfN}$ & Bundesamt für Naturschutz \\
\hline BMBF & Bundesministerium für Bildung und Forschung \\
\hline BMVEL & $\begin{array}{l}\text { Bundesministerium für Verbraucherschutz, } \\
\text { Landwirtschaft }\end{array}$ \\
\hline CBD & Convention on Biological Diversity \\
\hline CCC & Commodity Credit Corporation \\
\hline CRP & Conservation Reserve Program \\
\hline EAGFL & Europäischer Ausrichtungs- und Garantiefonds für die Landwirtschaft \\
\hline EBI & Environmental Benefits Index \\
\hline EG & Europäische Gemeinschaft \\
\hline EI & Erodibility Index \\
\hline EQIP & Environmental Quality Incentives Program \\
\hline $\mathrm{EU}$ & Europäische Union \\
\hline EWG & Europäische Wirtschaftsgemeinschaft \\
\hline FACT & Food, Agriculture, Conservation and Trade Act \\
\hline FAIR & Federal Agricultural Improvement and Reform Act \\
\hline FSA & Farm Service Agency \\
\hline GAK & Gemeinschaftsaufgabe Agrarstruktur und Küstenschutz \\
\hline GAP & Gemeinsame Agrarpolitik \\
\hline ha & Hektar \\
\hline HGrG & Haushaltsgrundsätzegesetz \\
\hline Hrsg. & Herausgeber \\
\hline InVeKos & Integriertes Verwaltungs- und Kontrollsystem \\
\hline KOM & Europäische Kommission \\
\hline Max. & Maximum \\
\hline MBI & Market-based Instruments Pilots Program \\
\hline Min. & Minimum \\
\hline NAP & National Action Plan for Salinity and Water Quality \\
\hline NAU & Niedersächsisches Agrarumweltprogramm \\
\hline NBER & National Bureau of Economic Research \\
\hline NECMA & North East Catchment Management Authority \\
\hline
\end{tabular}




$\begin{array}{ll}\text { Nr. } & \text { Nummer } \\ \text { NRCS } & \text { National Resource Conservation Service } \\ \text { OECD } & \text { Organisation for Economic Co-Operation and Development } \\ \text { PROLAND } & \begin{array}{l}\text { Programm zur Entwicklung der Landwirtschaft und des ländlichen } \\ \text { Raums }\end{array} \\ \text { SRU } & \text { Sachverständigenrat für Umweltfragen } \\ \text { Std.abw. } & \text { Standardabweichung } \\ \text { USDA } & \text { United States Department of Agriculture } \\ \text { USGAO } & \text { United States General Accounting Office } \\ \text { VO } & \text { Verordnung } \\ \text { VOB } & \text { Verdingungsverordnung für Bauleistungen } \\ \text { WRP } & \text { Wetlands Reserve Program }\end{array}$




\section{Einleitung und Aufbau}

\subsection{Einleitung}

Die nationale und internationale Agrarumweltpolitik ist derzeit durch grundlegende Weiterentwicklungen geprägt, wobei der Multifunktionalität der landwirtschaftlichen Produktion eine zentrale Rolle zukommt (Heissenhuber und Lippert 2000, S. 249; OECD 2001a; Latacz-Lohmann und Hodge 2003, S. 132-134). Dieser multifunktionale Charakter ist dadurch gekennzeichnet, dass die Landwirtschaft neben der Produktion klassischer Agrarerzeugnisse, wie Nahrungsmittel und Rohstoffe, unter anderem auch einen weitreichenden Einfluss auf den Umwelt- und Naturschutz sowie den Erhalt der Biodiversität ausübt (OECD 2001a, S. 27; Marggraf 2003, S. 507). ${ }^{1}$

Dem spezifischen Schutz der Biodiversität, in Form ihres In-situ-Erhalts und ihrer nachhaltigen Nutzung, wurde mit dem im Jahr 1992 verabschiedeten und international verbindlichen Übereinkommen über die Biologische Vielfalt (CBD) ${ }^{2}$ seitens der internationalen Staatengemeinschaft ein besonderer Stellenwert eingeräumt (Marggraf et al. 2005, S. 94-95). Durch die Unterzeichnung der Biodiversitätskonvention haben sich auch die EU-Mitgliedsstaaten verpflichtet, entsprechende politische Maßnahmen zu ergreifen und notwendige Anreize für Landbewirtschafter zu setzen. $^{3}$

Zur Erreichung der Biodiversitätsschutzziele auf landwirtschaftlich genutzten Flächen und einer grundsätzlichen Förderung einer umweltschonenden Bewirtschaftung wurden insbesondere mit der EU-Agrarreform aus dem Jahr 1992 Agrarumweltprogramme aufgelegt, welche sich bis heute $\mathrm{zu}$ den wesentlichen Aspekten der europäischen und nationalen Agrarpolitik entwickelt haben (Marggraf et al. 2005, S. 93). ${ }^{4}$

1 Entsprechende ökologische Leistungen der Landwirtschaft erfahren zudem eine positive gesellschaftliche Wertschätzung (Heissenhuber und Lippert 2000, S. 249-250).

${ }^{2}$ CBD steht für „Convention on Biological Diversity“.

${ }^{3}$ Insbesondere durch die Artikel 6, 8 und 11 der CBD.

4 In Deutschland wurden im Jahr 2000 durch Agrarumweltprogramme rund $25 \%$ der landwirtschaftlich genutzten Fläche honoriert und im Zuge dessen seitens der EU, des Bundes und der Länder insgesamt ca. 531 Mill. $€$ an die teilnehmenden Betriebe ausgezahlt (Marggraf et al. 2005, S. 93). 
Doch wie erfolgt die Honorierung der Landwirte ${ }^{5}$ innerhalb von Agrarumweltprogrammen und im Zuge dessen die Anreizsetzung zur Produktion ökologischer Leistungen? Sofern die Erbringung einer spezifischen Umweltleistung Eingang in ein Agrarumweltprogramm gefunden hat, ist eine Honorierung durch die Zahlung einheitlicher Prämien vorgesehen. ${ }^{6}$

Dies ist aus ökonomischer Sicht maßgeblich dahingehend zu kritisieren, dass bei einer Einheitsprämie die individuellen Unterschiede der Produktionsbedingungen eines landwirtschaftlichen Betriebs keine Beachtung finden (Latacz-Lohmann und Van der Hamsvoort 1997, S. 407-408; Plankl 1999, S. 164-165). Das Prinzip "gleiches Geld für gleiche Leistung « mag auf den ersten Blick plausibel und "gerecht« erscheinen. Die ökonomische Schwäche des Ansatzes verdeutlicht jedoch der Aspekt, dass die Erbringung einer definierten Umweltleistung bei jedem Landwirt in der Regel unterschiedliche Kosten verursacht. Bei einer Honorierung über eine administrativ fixierte Einheitsprämie erfahren diese Kostenunterschiede keinerlei Berücksichtigung, was letztlich dazu führt, dass öffentliche Mittel undifferenziert und ineffizient eingesetzt werden (Holm-Müller et al. 2002, S. 112; SRU 2002, S. 96-98).

Diese Probleme werden zunehmend auch politisch erkannt und Ansätze zu ihrer Überwindung haben im Rahmen der Verordnung (EG) Nr. 1698/2005 Eingang in die EU-Agrarreform für den Zeitraum 2007 bis 2013 gefunden. Infolgedessen sind weitreichende Auswirkungen auf die zukünftige Ausgestaltung von Agrarumweltprogrammen zu erwarten, wobei für die vorliegende Untersuchung insbesondere zwei in Artikel 39 der Verordnung (EG) Nr. 1698/2005 bestimmte grundlegende Veränderungen der Prämienfestlegung relevant sind. Es werden darin erstmals die Möglichkeiten aufgenommen, dass

a) Zahlungen für Agrarumweltmaßnahmen über eine Ausschreibung anstelle einer einheitlichen Prämie erfolgen können und

b) Transaktionskosten ein Kriterium zur Bemessung von Zahlungen an Landwirte darstellen.

\footnotetext{
${ }^{5}$ Aus Gründen der einfacheren Lesbarkeit wird auf die explizite Nennung der weiblichen Form verzichtet. Sie ist selbstverständlich ebenfalls gemeint.

${ }^{6}$ Bis Ende 2006 auf Grundlage der Verordnung (EG) Nr. 1257/1999.
} 
Hier setzt die vorliegende praxisbezogene Arbeit an. Ihre maßgeblichen Ziele stellen im Hinblick auf diese Weiterentwicklung der Agrarumweltpolitik die praktische Erprobung von Ausschreibungen und die Diskussion der Möglichkeiten einer Einbeziehung von Transaktionskosten in die Zahlungen an Landwirte dar. Im Zuge dessen wird mit der Nutzung eines Ausschreibungsverfahrens die Integration marktwirtschaftlicher Elemente zur Honorierung von Umweltleistungen unter Effizienzgesichtspunkten verfolgt, wobei der Schwerpunkt auf dem Schutz der pflanzlichen Biodiversität liegt. ${ }^{7}$

Zur Analyse dieser Neuausrichtung der Agrarumweltpolitik und ihrer zukünftig bestmöglichen praktischen Ausgestaltung wird neben den sich herausbildenden Angebotspreisen auf die durch das ihr zugrunde liegende Effizienzziel geprägte Transaktionskostenökonomik zurückgegriffen. Hierbei stehen sowohl die Beziehung zwischen Agrarverwaltung und Landwirten als auch die Ermittlung und Bewertung der Transaktionskosten der Landwirte im Mittelpunkt. Eine entsprechende Fokussierung auf die Aspekte der Landwirte ist darin begründet, dass durch die Einführung eines Ausschreibungsverfahrens in Form der eigenständigen Preiskalkulation weitreichende neue Herausforderungen an das unternehmerische Handeln zu erwarten sind. Zudem ist die Transaktionskostenbewertung auf Seiten der Landwirte im Gegensatz zur Agrarverwaltung auf nationaler wie auch auf internationaler Ebene noch durch grundlegenden Forschungsbedarf gekennzeichnet (Falconer und Whitby 1999, S. 26-27; OECD 2001b, S. 4-5).

\subsection{Aufbau}

In Kapitel Zwei wird einführend der zugrunde liegende agrarumweltpolitische Rahmen betrachtet. Hierbei erfolgt beginnend mit der EU-Agrarreform aus dem Jahr 1992 die Einordnung von Agrarumweltprogrammen in die europäische und nationale Agrarumweltpolitik und es werden darauf aufbauend sowohl bestehende Kritikpunkte an Agrarumweltprogrammen aufgezeigt als auch bereits absehbare politische Ansätze zu ihrer Weiterentwicklung einbezogen.

\footnotetext{
${ }^{7}$ In diesem Kontext kann Biodiversität als ein ökonomisches Gut aufgefasst werden, da biologische Vielfalt sowohl in umfassender Weise einen Beitrag zur menschlichen Bedürfnisbefriedigung leistet und demzufolge einen ökonomischen Nutzen impliziert als auch ein knappes Gut darstellt (Baumgärtner 2002, S. 73-74).
} 
Die praktische Grundlage dieser Arbeit bildet die Umsetzungsphase eines interdisziplinären Forschungsprojekts, welches in Kapitel Drei anhand seiner Komponenten und einer Übersicht über den Ablauf der hier relevanten Untersuchungen vorgestellt wird.

Die theoretische Basis der nachfolgenden Analyse des Einsatzes von Ausschreibungen zur Honorierung von Umweltleistungen stellt die in Kapitel Vier betrachtete Transaktionskostenökonomik dar.

Nach einem Exkurs über die Entwicklung des Ansatzes und ersten grundlegenden begrifflichen Abgrenzungen wird der Analyserahmen eingeführt. Dieser beinhaltet die zugrunde gelegten Verhaltensannahmen, die zu unterscheidenden Dimensionen von Transaktionen und die daraus resultierenden Ansätze einer effizienten Beherrschung und Überwachung von Transaktionsbeziehungen. Im Anschluss werden weitergehende Definitionsversuche von Transaktionskosten vorgenommen und die in der Literatur vorherrschende Kritik an der Transaktionskostenökonomik aufgezeigt.

Das ökonomische Instrument einer Ausschreibung und die spezifische Anwendung für die Honorierung ökologischer Leistungen ist Bestandteil des fünften Kapitels.

Vor dem Hintergrund der Charakterisierung von Ausschreibungen als ein Spezialfall einer Auktion beginnt das Kapitel mit einer Einführung in die relevanten auktionstheoretischen Grundlagen. Im Anschluss richtet sich der Fokus explizit auf Ausschreibungen für Umweltleistungen und nach einer Diskussion der grundlegenden Ausgestaltungsmöglichkeiten wird das im Rahmen dieser Arbeit gewählte Ausschreibungsdesign abgeleitet und erläutert. Abschließend werden drei bereits in der Praxis erprobte Ausschreibungsverfahren für Umweltleistungen vorgestellt und im Zuge dessen der Stand der Forschung dargelegt.

Als Bindeglied zwischen den theoretisch-konzeptionellen Ausführungen und den empirischen Untersuchungen erfolgt in Kapitel Sechs die transaktionskostenökonomische Analyse der durchführenden Agrarverwaltung und der teilnehmenden Landwirte innerhalb eines Ausschreibungsverfahrens.

Dazu wird die entsprechende Transaktionsbeziehung einführend in ihren Grundlagen erläutert und im Anschluss hinsichtlich der spezifischen Verhaltensannahmen der 
beteiligten Akteure und der Dimensionen der Transaktion analysiert. Unter Berücksichtigung dieser Ergebnisse wird anschließend die effiziente Ausgestaltung der Transaktionsbeziehung diskutiert und die Anwendbarkeit der innerhalb der Transaktionskostenökonomik in der Regel unterschiedenen Beherrschungs- und Überwachungsstrukturen untersucht. Abschließend erfolgt, unter Einbeziehung des der Arbeit zugrunde liegenden Ausschreibungsdesigns, eine zusammenfassende Diskussion der transaktionskostenökonomischen Analyse.

Im Anschluss wendet sich die Arbeit den empirischen Untersuchungen in der Modellregion zu, indem in Kapitel Sieben die im Zeitraum 2004/2005 und 2006 durchgeführten Ausschreibungen betrachtet werden.

Hierbei werden für beide Ausschreibungsverfahren, nach einer Übersicht über den zeitlichen Ablauf und die grundlegende Beiratsentscheidung, die Ausschreibungsergebnisse jeweils separat für die ökologischen Güter und anschließend zusammenfassend hinsichtlich der Angebotspreise diskutiert. Ergänzend werden auf der Grundlage von Befragungsergebnissen Aspekte der Preiskalkulation und des Bieterverhaltens der Landwirte einbezogen. Das Kapitel abschließend wird eine zusammenfassende Gegenüberstellung sowohl der Ausschreibungs- als auch der Befragungsergebnisse beider Ausschreibungsrunden durchgeführt.

Die Diskussion der für die Landwirte im Verlauf der Angebotsabgabe in beiden Ausschreibungsverfahren relevanten Transaktionskosten erfolgt im achten Kapitel. Nachdem einführend die bisherige Berücksichtigung von Transaktionskosten innerhalb von Agrarumweltprogrammen und der Stand der Forschung umrissen werden, wird die Definition der Transaktionskosten vorgenommen und das weitere methodische Vorgehen dargelegt. Anschließend werden die Transaktionskosten jeweils für die Ausschreibungen 2004/2005 und 2006 ermittelt und unter Einbeziehung von zusätzlichen Befragungsergebnissen sowohl zur Bewertung von Ausschreibungen als auch hinsichtlich der Möglichkeiten ihrer Einbeziehung in Zahlungen an Landwirte zusammenfassend diskutiert.

Nachdem in Kapitel Neun weitergehender Forschungsbedarf abgeleitet wird, schließt die Arbeit in Kapitel Zehn mit einem zusammenfassenden Fazit. 


\section{Agrarumweltpolitischer Rahmen}

Nachfolgend wird der agrarumweltpolitische Hintergrund dargestellt, welcher den rechtlichen Rahmen der weiteren Betrachtungen vorgibt und die Einbettung der Agrarumweltprogramme in die europäische und nationale Agrarpolitik umfasst.

Hierzu werden in Abschnitt 2.1 einführend die im Bereich der Agrarumweltpolitik maßgeblichen Regelungen und Entwicklungen skizziert. Anschließend erfolgt in Abschnitt 2.2 eine kritische Bewertung der aktuellen Agrarumweltpolitik und das Kapitel abschließend behandelt Abschnitt 2.3 derzeit absehbare Perspektiven für die Weiterentwicklung der europäischen und nationalen Agrarumweltpolitik.

\subsection{Agrarumweltprogramme}

Als zentraler Bestandteil der EU-Agrarreform aus dem Jahr 1992 wurden Agrarumweltprogramme durch die VO (EWG) Nr. 2078/92 ${ }^{8}$ verabschiedet und im Zuge dessen grundlegende Schritte in Richtung einer umweltverträglichen Landwirtschaft vollzogen. Fortgeführt und ausgebaut wurde die Agrarpolitik durch die AGENDA 2000 und damit verbunden im Bereich der Agrarumweltpolitik durch die VO (EG) Nr. 1257/1999 ${ }^{9}$, die Agrarumweltmaßnahmen zu den wesentlichen Elementen der so genannten 2. Säule der „Gemeinsamen Agrarpolitik“ (GAP) ${ }^{10}$ der EU gemacht hat. Diese 2. Säule umfasst unter anderem die ländliche Entwicklungspolitik und soll durch die Bereitstellung von Agrarumweltprogrammen zur Verwirklichung der Ziele der Agrar- und Umweltpolitik der EU beitragen und den Landwirten Anreize zur Durchführung entsprechender Maßnahmen bieten.

Nach Artikel 22 der VO (EG) Nr. 1257/1999 sind Zahlungen in Agrarumweltmaßnahmen definiert als „Beihilfen für landwirtschaftliche Produktionsverfahren, die auf den Schutz der Umwelt und die Erhaltung des

\footnotetext{
8 Verordnung (EWG) Nr. 2078/92 des Rates vom 30. Juni 1992 für umweltgerechte und den natürlichen Lebensraum schützende landwirtschaftliche Produktionsverfahren.

${ }^{9}$ Verordnung (EG) Nr. 1257/1999 des Rates vom 17. Mai 1999 über die Förderung der Entwicklung des ländlichen Raums durch den Europäischen Ausrichtungs- und Garantiefonds für die Landwirtschaft (EAGFL) und zur Änderung bzw. Aufhebung bestimmter Verordnungen.

10 Die EU-Agrarpolitik unterteilt sich in zwei Säulen: Die 1. Säule umfasst den Bereich der Marktordnungen und die 2. Säule lässt sich zu den drei Schwerpunkten Verbesserung der Wettbewerbsfähigkeit der Agrarwirtschaft, Stärkung der ländlichen Entwicklung und Ausbau der Umwelt- und Ausgleichsmaßnahmen zusammenfassen.
} 
ländlichen Lebensraums ausgerichtet sind“ (Art. 22, Abs. 1, VO (EG) Nr. 1257/1999).

Im Rahmen derartiger Programme können Landwirte für die von ihnen erbrachten ökologischen Leistungen einen finanziellen Ausgleich erhalten, der sich an den mit der Umweltleistung verbundenen zusätzlichen Kosten und Einkommensverlusten orientiert. Diese Ausgleichszahlung erfolgt für alle im Geltungsbereich eines Agrarumweltprogramms befindlichen Landwirte durch eine einheitliche Prämie, welche eine über die reine Kostendeckung hinausgehende Anreizkomponente von bis zu 20 \% beinhalten kann (Art. 24, Abs. 1, VO (EG) Nr. 1257/1999; Art. 17-18, VO (EG) Nr. 1750/1999 ${ }^{11}$ ).

Die administrative Festlegung der Prämienzahlung für die Teilnahme an einem Agrarumweltprogramm ist bis zum Ende des Jahres 2006 einheitlich nach dem Schema: Zusatzkosten + Einkommensverluste + Anreizkomponente von bis zu $20 \%$ strukturiert, wobei die Landwirte eine jährliche Auszahlung der Prämien erhalten.

Die Finanzierung der Agrarumweltprogramme wird sowohl aus nationalen Mitteln als auch kofinanziert durch die EU vorgenommen. Entsprechend des in der VO (EG) Nr. 1257/1999 geregelten „Europäischen Ausrichtungs- und Garantiefonds für die Landwirtschaft“ (EAGFL) können Agrarumweltprogramme eine Kofinanzierung von 50-75 \% durch die EU erhalten. Um diese Kofinanzierung zu ermöglichen, setzt die VO (EG) Nr. 1257/1999 eine mindestens fünfjährige vertragliche Verpflichtung des Landwirts zur Durchführung von Umweltmaßnahmen voraus. ${ }^{12}$

In Deutschland stellt die „Gemeinschaftsaufgabe Verbesserung der Agrarstruktur und des Küstenschutzes“ (GAK) das Hauptinstrument der Agrarpolitik dar, innerhalb dessen Bund und Länder die Schwerpunkte ihrer Politik vereinbaren.

Der Bund nutzt die ihm gebotenen Möglichkeiten in der Form, dass eine Förderung der markt- und standortangepassten Landbewirtschaftung verfolgt wird. Der überwiegende Teil der Agrarumweltmaßnahmen wird aufgrund der föderalen

\footnotetext{
11 Verordnung (EG) Nr. 1750/1999 der Kommission vom 23. Juli 1999 mit Durchführungsvorschriften zur Verordnung (EG) Nr. 1257/1999 des Rates über die Förderung und Entwicklung des ländlichen Raums durch den Europäischen Ausrichtungs- und Garantiefonds für die Landwirtschaft (EAGFL).

12 Mit den Beschlüssen zur AGENDA 2000 standen für den Förderzeitraum 2000-2006 in Deutschland rund 18 Milliarden $€$ aus EU-, Bundes- und Landesmitteln für die 2. Säule der GAP zur Verfügung (BMVEL 2004, S. 95).
} 
Struktur Deutschlands seitens der Bundesländer angeboten, woraus resultiert, dass sich die Agrarumweltprogramme einzelner Länder oftmals grundlegend voneinander unterscheiden und jeweils spezifische Schwerpunktsetzungen beinhalten. ${ }^{13}$

Sämtliche Zahlungen innerhalb der GAP sind an die Einhaltung zweier Bewirtschaftungsvorgaben gebunden. Zum einen der Erfüllung der „guten landwirtschaftlichen Praxis“ und zum anderen den sich aus den „Cross Compliance $^{\text {“14 }}$ ergebenden Anforderungen. Sie stellen die praxisübliche Form der Landwirtschaft dar und sichern ein Mindestmaß an Berücksichtigung von für den Umwelt- und Tierschutz relevanten Vorgaben. Daraus ergibt sich die Notwendigkeit, dass Zahlungen im Rahmen von Agrarumweltprogrammen nur gerechtfertigt sind, wenn die durch sie geförderten Maßnahmen über die Bestimmungen der guten landwirtschaftlichen Praxis (Art. 23, Abs. 2, VO (EG) 1257/1999) und der Cross Compliance (Kap. I, VO (EG) Nr. 1782/2003) hinausgehen.

Die Kontrolle der zum Erhalt von Beihilfezahlungen berechtigenden Umweltleistungen ist mit dem Ziel der Vermeidung ungerechtfertigter Zahlungen einheitlich durch das so genannte „Integrierte Verwaltungs- und Kontrollsystem“ (InVeKoS) festgelegt, welches in der VO (EG) Nr. 2419/1999 ${ }^{15}$ und der VO (EG) Nr. $1782 / 2003^{16}$ geregelt ist.

\subsection{Kritikpunkte derzeitiger Agrarumweltprogramme}

Im Rahmen unterschiedlichster Evaluierungen von Agrarumweltprogrammen (Hofmann et al. 1995; KOM 1998; Plankl 1999; Wilhelm 1999; Klein und Sutherland 2003) wurden diverse Problembereiche aufgezeigt. Die hier relevante und

\footnotetext{
${ }^{13}$ In Niedersachsen erfolgt die Umsetzung der Agrarumweltmaßnahmen beispielsweise über den Entwicklungsplan „PROLAND NIEDERSACHSEN - Programm zur Entwicklung der Landwirtschaft und des ländlichen Raums“ (PROLAND), der im September 2000 von der Europäischen Kommission genehmigt wurde. Das PROLAND-Programm umfasst drei Förderschwerpunkte, wobei die Agrarumweltmaßnahmen in den Förderschwerpunkt III: „Agrarumwelt- und Ausgleichsmaßnahmen sowie Maßnahmen zum Schutz der Umwelt“ eingebettet sind.

${ }^{14}$ Die Regelungen zu den „Cross Compliance“ werden teilweise auch als „Einhaltung anderweitiger Verpflichtungen“ bezeichnet. Gebräuchlich ist jedoch die Bezeichnung „Cross Compliance“, welche ebenso im Folgenden verwendet wird.

15 Verordnung (EG) Nr. 2419/2001 der Kommission vom Dezember 2001 mit Durchführungsbestimmungen zum mit der Verordnung (EWG) Nr. 3508/92 des Rates vom 27. November 1992 eingeführten Integrierten Verwaltungs- und Kontrollsystems für bestimmte gemeinschaftliche Beihilferegelungen.

${ }^{16}$ Verordnung (EG) Nr. 1782/2003 des Rates vom 29. September 2003 mit gemeinsamen Regeln für Direktzahlungen im Rahmen der Gemeinsamen Agrarpolitik und mit bestimmen Stützungsregelungen für Inhaber landwirtschaftlicher Betriebe.
} 
sich auf die geringe ökologische und ökonomische Effizienz und eine mangelnde gesellschaftliche Legitimation der Agrarumweltpolitik beziehende Kritik wird nun skizziert.

I. Die mangelnde gesellschaftliche Akzeptanz von Agrarumweltprogrammen ist maßgeblich in der fehlenden Berücksichtigung von Bevölkerungspräferenzen und regionalem Expertenwissen begründet, da die Programmziele zentral durch die zuständige Planungsbehörde festgelegt werden. Insbesondere vor dem Hintergrund der Kürzung staatlicher Transferzahlungen kann eine derartige Ausgestaltung zu einem dauerhaften Legitimationsproblem von Zahlungen für durch die Landwirtschaft erbrachte Umweltleistungen führen (Wilhelm 1999, S. 196-197; Marggraf et al. 2005, S. 95-97).

II. Aus ökologischer Sicht ist an gegenwärtigen Agrarumweltprogrammen eine überwiegend handlungsorientierte Ausrichtung zu kritisieren, im Rahmen derer Inhaber landwirtschaftlicher Betriebe ausschließlich für die Durchführung einer vorab festgelegten Maßnahme durch eine Prämienzahlung honoriert werden (Bertke et al. 2003a, S. 243-245). Infolgedessen kann das Erreichen der intendierten Umweltleistung nicht als gesichert angesehen werden und es existiert kein direkter Zusammenhang zwischen Honorierung und Ergebnis (Gerowitt und Marggraf 2001, S. 18-19).

Neben der fehlenden Überprüfung der Ergebnisse agrarumweltpolitischer Maßnahmen ist an einer handlungsorientierten Honorierung zu kritisieren, dass sich Landwirte mit einer Vielzahl von Reglementierungen und Bewirtschaftungsvorgaben konfrontiert sehen. Diese Einschränkungen von Handlungsalternativen beinhalten für die Landwirte keine Anreize über die Erfüllung der Vertragsinhalte hinausgehende Umweltleistungen zu erbringen oder ihr individuelles Wissen bei der Erreichung von Umweltzielen einzubringen (SRU 1996, S. 92).

III. Aus ökonomischer Sicht ist neben einem wenig leistungsbezogenen Einsatz öffentlicher Mittel die derzeitige Honorierung von Umweltleistungen durch eine einheitliche Prämienzahlung zu kritisieren (Latacz-Lohmann und Van der Hamsvoort 1997, S. 407-408; Marggraf et al. 2005, S. 96-97). 
$\mathrm{Zu}$ beanstanden ist an der Zahlung pauschaler Prämien vornehmlich ihre geringe volkswirtschaftliche Effizienz, da individuelle Anpassungskosten keine Berücksichtigung finden. Landwirte die in landwirtschaftlich eher ungünstigen Gebieten wirtschaften, können oftmals lediglich eine extensive Flächennutzung verfolgen, da die Standorte auch bei intensiver Bewirtschaftung keine Höchsterträge hervorbringen. Für eine Teilnahme an Agrarumweltprogrammen ${ }^{17}$ müssen diese Landwirte ihre Bewirtschaftung kaum bzw. gar nicht umstellen, können jedoch die gleiche finanzielle Entlohnung realisieren wie Landwirte in eher günstigen Gebieten. Für jene Landwirte hingegen ist eine zur Teilnahme an Agrarumweltprogrammen notwendige Umstellung der Bewirtschaftung mit wesentlich höheren Kosten verbunden und der Einkommensrückgang kann durch die einheitliche Prämie oftmals nicht kompensiert werden (Baudoux et al. 1997, S. 185-188; Latacz-Lohmann und Schilizzi 2005, S. 5). Eine Teilnahme an Agrarumweltprogrammen findet nicht statt, doch gerade hier könnte eine deutliche Umweltentlastung erreicht werden.

In der Folge kommt es einerseits zu Mitnahmeeffekten und andererseits zu einer zu geringen Teilnahme, mit der Konsequenz, dass begrenzte öffentliche Mittel nicht effizient eingesetzt werden und ein zu geringer ökologischer Nutzen realisiert wird (Plankl 1998, S. 45).

\subsection{Ansätze zur Weiterentwicklung der Agrarumweltpolitik}

Hinsichtlich der Entwicklung der Agrarumweltpolitik sind auf der rechtlichen Seite insbesondere zwei Ansätze zu nennen, die im Folgenden beschrieben werden und im Kontext der hier verfolgten Forschungsarbeit eine Verbesserung der Agrarumweltpolitik mit Impulsen zur zumindest teilweisen Überwindung der im vorherigen Abschnitt skizzierten Probleme darstellen.

I. Eine bereits umgesetzte nationale Weiterentwicklung stellen die in den GAKRahmenplan für den Zeitraum 2005 bis 2008 eingebetteten „Grundsätze für die Förderung einer markt- und standortangepassten Landbewirtschaftung“ als Teil der Agrarumweltförderung des Bundes dar.

In Abschnitt B - „Förderung extensiver Grünlandnutzung“ - werden Beihilfen ermöglicht, welche die Einführung und Beibehaltung extensiver

\footnotetext{
${ }^{17}$ Beispielsweise eine extensive Nutzung von Grünlandflächen.
} 
Grünlandbewirtschaftungsverfahren zur nachhaltigen Verbesserung der natürlichen und wirtschaftlichen Produktionsbedingungen sowie des Schutzes der Umwelt und des natürlichen Lebensraums zum Ziel haben. Von besonderem Interesse ist dort die Erhaltung pflanzengenetisch wertvoller Grünlandvegetation. Zwar erfolgt auch hier die Honorierung der umweltrelevanten Leistungen noch immer über eine einheitliche Prämie, jedoch wird durch ein definiertes ökologisches Ziel der Schritt weg von der Handlungsorientierung hin zu einer Ergebnisorientierung vollzogen. Die ökologische Zieldefinition schreibt für eine Förderung das Vorkommen von mindestens vier Kennarten aus einem landes- oder regionalspezifisch erstellten Katalog von 20 bis 40 krautigen Pflanzen vor.

Zur Honorierung dieser Umweltleistung sieht der vom Bund-Länder-Ausschuss für Agrarstruktur und Küstenschutz am 18. November 2004 beschlossene GAKRahmenplan 2005 - 2008 die Zahlung von $130 €$ pro Hektar Grünlandvegetation als einheitliche Prämie vor. ${ }^{18}$

II. Einen deutlich umfassenderen Ansatz zur Überwindung der Probleme aktueller Agrarumweltprogramme mit der Perspektive 2007 bis 2013 stellt die am 20. September 2005 beschlossene und ab Januar 2007 gültige „Verordnung über die Förderung der Entwicklung des ländlichen Raums durch den Europäischen Landwirtschaftsfonds für die Entwicklung des ländlichen Raums (ELER)“ (VO (EG) Nr. 1698/2005) dar. ${ }^{19}$ Die ELER-Verordnung zeigt Ansätze für eine Neuorientierung der europäischen Agrarumweltpolitik auf und lässt eine entsprechende Weiterentwicklung erwarten, wobei hier die folgenden drei Aspekte zu betrachten sind:

a) Artikel 7 der ELER-Verordnung greift den Gesichtspunkt der Subsidiarität auf und den Mitgliedsstaaten wird die Verantwortung für die Umsetzung von Programmen der Entwicklung des ländlichen Raums auf der geeigneten Gebietsebene und unter Berücksichtigung der institutionellen Voraussetzungen zugeteilt.

\footnotetext{
${ }^{18}$ In Kapitel Sieben der vorliegenden Arbeit wird diese Prämienzahlung erneut aufgegriffen und als ein Referenzwert zur Untersuchung der praktischen Effizienzvorteile des Einsatzes von Ausschreibungsverfahren im Vergleich mit einheitlichen Prämienzahlungen herangezogen.

19 Nachfolgend kurz als ELER-Verordnung bezeichnet. Somit wird die Verordnung (EG) Nr. 1257/1999 ab 01. Januar 2007 durch die ELER-Verordnung ersetzt.
} 
b) In Artikel 39 der ELER-Verordnung ist aufgenommen, dass Zahlungen im Rahmen von Agrarumweltmaßnahmen „[...] über eine Ausschreibung unter Anwendung von wirtschaftlichen und ökologischen Effizienzkriterien [...]“ erfolgen können. Die Verpflichtungen sind in der Regel für einen Zeitraum von fünf bis sieben Jahren einzugehen und sofern es für die Erreichung der Umweltziele gerechtfertigt ist, soll es auch anderen Landbewirtschaftern als Landwirten möglich sein, Zahlungen für Agrarumweltmaßnahmen zu erhalten. Diese Zahlungen erfolgen jährlich und können bestimmte im Anhang der ELER-Verordnung festgesetzte Beihilfehöchstbeträge nicht übersteigen. ${ }^{20}$ Im Hinblick auf das einem Ausschreibungsverfahren zugrunde zu legende Ausschreibungsdesign beinhaltet die ELER-Verordnung keinerlei Vorgaben.

c) Zudem werden in Artikel 39 der ELER-Verordnung sowohl im Rahmen eines Ausschreibungsverfahrens als auch einer einheitlichen Prämienzahlung Transaktionskosten als ein Kriterium zur Bemessung entsprechender Zahlungen innerhalb von Agrarumweltmaßnahmen eingeführt. Somit erfolgt ebenfalls eine Anpassung der administrativen Prämienfestlegung, da die Berücksichtigung einer zusätzlichen Anreizkomponente von 20 \% nunmehr entfällt und die Einbeziehung von Transaktionskosten ermöglicht wird. Die Festlegung einer einheitlichen Prämienzahlung erfolgt dementsprechend ab 2007 nach dem Schema: Zusatzkosten + Einkommensverlust + Transaktionskosten. Jedoch sieht die ELER-Verordnung weder eine Definition von Transaktionskosten noch eine Methode zu ihrer Ermittlung vor.

Für die anschließenden Betrachtungen wird demzufolge innerhalb des sich aus der ELER-Verordnung ergebenden Forschungsbedarfs hauptsächlich der Artikel 39 aufgegriffen und mit dem Fokus auf der praktischen Erprobung von Ausschreibungen zur Honorierung von Umweltleistungen und der Ermittlung und Bewertung von Transaktionskosten der Landwirte diskutiert.

\footnotetext{
${ }^{20}$ Im Einzelnen betragen die Beihilfehöchstsätze für einjährige Kulturen $600 €$ pro Hektar, für mehrjährigen Sonderkulturen $900 €$ pro Hektar und für sonstige Flächennutzungen $450 €$ pro Hektar.
} 


\section{Das Forschungsprojekt}

Die im Rahmen der vorliegenden Arbeit durchgeführten Untersuchungen waren in das interdisziplinäre Gesamtprojekt BIOPLEX zur Erforschung des Zusammenhangs zwischen Biodiversität und räumlicher Komplexität in Agrarlandschaften eingebunden, welches in einer Kooperation der Justus-Liebig-Universität Gießen und der Georg-August-Universität Göttingen durchgeführt wurde. ${ }^{21}$

Die hier relevante praktische Umsetzung des Forschungsprojekts erfolgte im Rahmen der zweiten Projektphase im Zeitraum 2004 bis 2006, mit dem Ziel die Problemfelder derzeitiger Agrarumweltprogramme in einem umfassenden und konzeptionell abgestimmten Ansatz zu überwinden.

Zur Einordnung der Arbeit in den Gesamtkontext wird der sich grundlegend vom Status quo der Agrarumweltprogramme unterscheidende Ansatz nachfolgend in seinen Grundzügen erläutert. Das Honorierungskonzept ist ergebnisorientiert ausgerichtet, beinhaltet in Form eines Ausschreibungsverfahrens die im Rahmen der Honorierung ökologischer Leistungen größtmögliche Einbeziehung marktwirtschaftlicher Strukturen und ist unter Berücksichtigung des Subsidiaritätsprinzips regional verankert. Zudem ermöglicht es die Beteiligung von lokalen Bevölkerungspräferenzen bei der ökologischen Zielfindung (Fischer et al. 2003, S. 390-393; Marggraf et al. 2005, S. 100-102).

Im weiteren Verlauf dieses Kapitels gibt Abschnitt 3.1 einen Überblick über die Komponenten des Honorierungssystems und in Abschnitt 3.2 erfolgt eine zusammenfassende Darstellung der Umsetzungsphase, im Zuge derer die Modellregion und der Ablauf der praktischen Erprobung im Mittelpunkt stehen.

21 Die Finanzierung erfolgte im Rahmen des Forschungsschwerpunkts BIOLOG des Bundesministeriums für Bildung und Forschung. Das hier betrachtete Forschungsprojekt „Ergebnisorientierte Honorierung ökologischer Leistungen der Landwirtschaft“ stellt innerhalb des BIOPLEX-Verbunds das sozioökonomische Teilprojekt dar und wurde am Forschungs- und Studienzentrum Landwirtschaft und Umwelt der Georg-August-Universität Göttingen in der ersten Projektphase im Zeitraum 2000 bis 2003 initiiert. 


\subsection{Komponenten des Honorierungssystems}

Im Folgenden werden mit den ökologischen Gütern Grünland, dem Regionalen Beirat Northeim, der Bevölkerungsbeteiligung und dem Ausschreibungsverfahren die wesentlichen Komponenten des Honorierungssystems vorgestellt.

\subsubsection{Ergebnisorientierung - Die ökologischen Güter Grünland}

Im Gegensatz zu der überwiegenden Zahl der bestehenden Agrarumweltprogramme wurde mit diesem Honorierungskonzept eine ergebnisorientierte Honorierung der unternehmerischen Tätigkeit von Landwirten erprobt. Dabei erfolgt eine Maßnahme nicht entkoppelt von ökologischen Effekten, sondern anhand eindeutig definierter und umweltpolitisch als erstrebenswert eingeschätzter ökologischer Zielgrößen. Als ökologische Zielgrößen wurden ökologische Güter definiert, welche die Ergebnisse ökologischer Leistungen der Landwirtschaft darstellen (Bertke 2002, S. 6; Bertke 2005, S. 18-20).

$\mathrm{Zu}$ den betrachteten ökologischen Gütern lassen sich pflanzliche Artenvielfalt im Grünland, auf Äckern oder in Zwischenstrukturen zählen, die je nach Artenzusammensetzung unterschiedliche Qualitätsstufen aufweisen. ${ }^{22}$ Durch die Existenz ökologischer Güter auf landwirtschaftlich genutzten Flächen kann eindeutig der Schutz und die Förderung der floristischen Biodiversität als das Erreichen des intendierten Ergebnisses nachgewiesen werden (Bertke 2005, S. 18-20; Bertke et al. 2003, S. 243-245). Infolgedessen bildet die unmittelbare Verknüpfung der Honorierung von Landwirten mit den von ihnen erbrachten Umweltleistungen die Grundlage für einen effektiven und effizienten Einsatz öffentlicher Mittel in Agrarumweltprogrammen.

Um als Zielgrößen einer ergebnisorientierten Honorierung eingesetzt zu werden, müssen ökologische Güter anhand von floristischen Honorierungskriterien definiert werden und spezifische Anforderungen erfüllen. So ist es notwendig, dass sie eindeutig und rechtssicher nachgewiesen und kontrolliert werden können, positive ökologische Effekte implizieren und sowohl realisierbar als auch honorierbar sind (Bertke 2005, S. 18-19).

\footnotetext{
${ }^{22}$ Dies kann beispielsweise eine bestimmte Anzahl verschiedener Kräuterarten im Grünland sein.
} 
Da im Rahmen der Umsetzungsphase die Ausschreibung und Produktion der ökologischen Güter Grünland im Mittelpunkt standen, werden im Folgenden speziell die Aspekte der Definition und Kontrolle der Umweltleistungen beispielhaft am Grünland betrachtet. ${ }^{23}$

Für eine ergebnisorientierte Honorierung eignen sich ökologische Güter der pflanzlichen Biodiversität im Grünland maßgeblich dadurch, dass sie eindeutig zu identifizieren, leicht zu kontrollieren und einzelbetrieblich zuzuordnen sind. Dazu wurde in der ersten Projektphase ein Katalog von in der Modellregion vorkommenden ökologischen Gütern Grünland definiert, der in Form der Leistungsbeschreibung einen Teil der Ausschreibungsunterlagen bildet. ${ }^{24}$

Des Weiteren wird nach Bertke entsprechend der qualitativen Beschaffenheit der ökologischen Güter zwischen Basis- und Qualitätsgütern unterschieden (Bertke 2005, S. 20-22).

Basisgüter zeichnen sich durch die Erfüllung einer Mindestartenvielfalt aus, die über die Anforderungen der guten landwirtschaftlichen Praxis und der Cross Compliance hinausgeht und dadurch eine explizite Honorierung rechtfertigt. Qualitätsgüter weisen neben den für eine Honorierung notwendigen Mindestanforderungen weitere Eigenschaften auf, die einen jeweils höheren ökologischen Nutzen implizieren und damit eine höhere finanzielle Honorierung rechtfertigen. ${ }^{25}$

Innerhalb der Umsetzungsphase führte dies bei den ökologischen Gütern im Grünland zu der Unterteilung in die Güter „Grünland I“, „Grünland II“ und „Grünland III“, wobei Grünland I das Basisgut ist und die Güter Grünland II und Grünland III als Qualitätsgüter definiert sind. Das Gut Grünland III stellt das qualitativ hochwertigste ökologische Gut dar und repräsentiert in Folge dessen die größte Artenvielfalt und den höchsten ökologischen Nutzen (Bertke 2005, S. 70$72){ }^{26}$

\footnotetext{
${ }^{23}$ Für weitergehende Betrachtungen zur Herleitung, Definition und Kontrolle ökologischer Güter sei an dieser Stelle auf die Dissertation von Bertke (2005) verwiesen.

${ }^{24}$ Die Ausschreibungsunterlagen finden sich im Anhang dieser Arbeit.

${ }^{25}$ Beispielsweise das Auftreten von „Rote-Liste-Arten“.

${ }^{26}$ Eine Beschreibung der ökologischen Güter und der Anforderungen an ihre Kontrolle wird in Kapitel Sieben im Rahmen der Darstellung der Umsetzung und der Ergebnisse der Ausschreibungsverfahren vorgenommen.
} 


\subsubsection{Subsidiarität - Der Regionale Beirat Northeim}

Um auch im Bereich der Agrarumweltpolitik dem von der Europäischen Union in Artikel 5 des Vertrags zur Gründung der Europäischen Gemeinschaft verankerten Subsidiaritätsprinzip zu entsprechen, ${ }^{27}$ ist das Honorierungssystem, und damit verbunden die Entscheidungskompetenz, auf regionaler Ebene angesiedelt.

Das Subsidiaritätsprinzip besagt, dass zuerst die dezentralste Ebene Maßnahmen zur Problemlösung bzw. zur Entscheidungsfindung, Politikgestaltung und Politikumsetzung ergreifen soll und die jeweils übergeordnete Stelle nur dann gefordert ist, wenn untergeordnete Stellen die entsprechenden Aufgaben nicht erfüllen können. ${ }^{28}$ Somit wird der Ansatz verfolgt, bei der ökologischen Zielfindung das regionale Expertenwissen möglichst aller relevanten Interessengruppen durch eine Rückübertragung von Gestaltungs- und Entscheidungskompetenzen auf die regionale Ebene gleichberechtigt zu berücksichtigen (Marggraf et al. 2005, S. 100102; Fischer et al. 2003, S. 390-394).

Hierzu wurde in der Modellregion Landkreis Northeim (Südniedersachsen) der Regionale Beirat Northeim eingerichtet. Dieses regionale Expertengremium konstituiert sich aus Vertretern der Bereiche Naturschutz, Landwirtschaft, Grundeigentum und Kommunalpolitik und zielt auf einen Interessenausgleich der beteiligten Akteure ab (Bertke et al. 2002, S. 191-192; Hespelt und Bertke 2003, S. 344-345).

Da es sich bei den ökologischen Gütern um öffentliche Güter handelt - also ihre Nutzung durch ein Individuum die Nutzungsmöglichkeiten anderer Individuen nicht einschränkt (Prinzip der Nicht-Rivalität im Konsum) und kein Individuum von ihrer Nutzung ausgeschlossen werden kann (Prinzip der Nicht-Ausschließbarkeit vom Konsum) - artikuliert sich keine private Nachfrage und es bildet sich folglich für diese Umweltgüter kein entsprechender Markt heraus (Samuelson 1954, S. 387-389; Musgrave et al. 1994, S. 67-72). ${ }^{29}$

\footnotetext{
${ }^{27}$ In der durch den Vertrag von Nizza geänderten Version vom 21. Februar 2001; bis dahin wurde das Subsidiaritätsprinzip in Artikel 3b festgeschrieben.

${ }^{28}$ Für eine umfassende Betrachtung des Subsidiaritätsprinzips siehe unter anderem Lecheler (1993) und Garbe (1994).

29 Dies ist darin begründet, dass wenn niemand von der Verwendung eines öffentlichen Guts ausgeschlossen werden kann und keine Rivalität der Verwendung existiert, auch kein Individuum bereit ist, für die Bereitstellung des Umweltguts zu zahlen (Trittbrettfahrer-Problem), was zur Folge hat, dass keine effiziente Allokation über Märkte erfolgt (Cansier 1993, S. 18-24; Wicke 1993, S. 41).
} 
Besteht jedoch ein staatliches Interesse an der Bereitstellung oder Erhaltung öffentlicher Güter, wie hier der durch die ökologischen Güter repräsentierten floristischen Biodiversität, muss eine politisch erwünschte Nachfrage von Seiten des Staates koordiniert erfolgen (Pommerehne und Röhmer 1992, S. 171-172). Diese Rolle übernimmt der Regionale Beirat Northeim und beschließt auf der Grundlage einer Geschäftsordnung nach dem Mehrheitsprinzip, wie das zur Verfügung stehende Budget auf die ökologischer Güter aufgeteilt wird. ${ }^{30}$ Im Anschluss und unter Berücksichtigung der Beiratsentscheidung schreibt die Agrarverwaltung die Produktion der Umweltleistungen aus und führt das Ausschreibungsverfahren durch. $^{31}$

\subsubsection{Partizipation - Die regionalen Bevölkerungspräferenzen}

In der gegenwärtigen Agrarumweltpolitik erfolgt die Festlegung ökologischer Ziele ohne die explizite Einbeziehung von gesellschaftlichen Präferenzen (Marggraf et al. 2005, S. 99-100). Dies widerspricht insbesondere der inhaltlichen Ausrichtung der Artikel 8 und 10 des Übereinkommens über die Biologische Vielfalt und den Prinzipien 1, 11 und 12 der im Ökosystemaren Ansatz ${ }^{32}$ der Biodiversitätskonvention implementierten Prinzipien.

So wird im Ökosystemaren Ansatz und dem Übereinkommen über die biologische Vielfalt eine Zielsetzung durch gesellschaftliche Entscheidungsprozesse, die Einbeziehung aller relevanten Gruppierungen der Gesellschaft und die Berücksichtigung jeder Art von relevanten Informationen gefordert (Der Ökosystemare Ansatz 2002 und CBD 1992). Demzufolge können und sollen Biodiversitätsschutzziele nicht ausschließlich von Experten und aus objektivnaturwissenschaftlicher Sicht festgelegt werden. Vielmehr gilt es, die subjektiven Interessen der Bevölkerung an der Erhaltung biologischer Vielfalt ebenfalls zu berücksichtigen und die ökologische Zielfindung auf Grundlage eines gesellschaftlichen Konsenses anzustreben (Wood 2000, S. 3-34).

\footnotetext{
30 Zur vertieften Betrachtung der Regionalisierung von Agrarumweltmaßnahmen am Beispiel des Regionalen Beirats Northeim sei an dieser Stelle auf die Dissertation von Hespelt (2005) verwiesen.

${ }^{31}$ Im Rahmen des Forschungsprojekts wurde die Durchführung des Ausschreibungsverfahrens von den Projektmitarbeitern der Universität Göttingen übernommen.

${ }^{32}$ Der Ökosystemare Ansatz beinhaltet zwölf von der Vertragsstaatengemeinschaft empfohlene Prinzipien einer ganzheitlichen Herangehensweise für den Umgang mit natürlichen Ressourcen.
} 
Zudem ist $\mathrm{zu}$ erwarten, dass eine derartige Einbeziehung regionaler Bevölkerungspräferenzen zu einer vertieften Auseinandersetzung mit der zugrunde liegenden Thematik und der in Artikel 13a der Biodiversitätskonvention geforderten gesellschaftlichen Bewusstseinsbildung führt. Diese umfasst sowohl ein höheres Wissen und eine höhere Akzeptanz gegenüber landwirtschaftlichen Themen (Marggraf et al. 2005, S. 95) als auch ein notwendiges kritisches Hinterfragen der Finanzströme im Agrarbereich und der damit einhergehenden staatlichen Rechtfertigung im Hinblick auf eine alternative Verwendung der zur Diskussion stehenden öffentlichen Mittel.

Mit dem Anspruch eine gesellschaftlich legitimierte Zielsetzung zu ermöglichen und den Anforderungen der Biodiversitätskonvention zu genügen, wurde in diesem Forschungsprojekt der Ansatz verfolgt, bei der ökologischen Zielfindung neben Expertenwissen auch regionale Bevölkerungspräferenzen $\mathrm{zu}$ berücksichtigen (Marggraf et al. 2005, S. 96). ${ }^{33}$

\subsubsection{Effizienz - Das Ausschreibungsverfahren}

Die Honorierung ökologischer Leistungen der Landwirtschaft erfolgt gegenwärtig ohne die Einbeziehung marktlicher Anreize und auf der Grundlage einer administrativen Preissetzung. Bei einer derartigen einheitlichen Prämie ist zu erwarten, dass es - verglichen mit einem Ausschreibungsverfahren - zu einem weniger effizienten Einsatz öffentlicher Mittel kommt (Holm-Müller et al. 2002, S. 112). Dies ist darin begründet, dass bei einer Einheitsprämie die individuelle Kostenstruktur eines landwirtschaftlichen Betriebs keine Berücksichtigung findet und alle Landwirte eine einheitliche Entlohnung erhalten (Plankl 1999, S. 164-165).

Bei einem Ausschreibungsverfahren ist es demgegenüber die Aufgabe der Landwirte ein freiwilliges Angebot abzugeben, welche Entlohnung sie für die Erbringung einer ökologischen Leistung als angemessen ansehen. Im Zuge dieser Preiskalkulation ist davon auszugehen, dass sich die Landwirte an ihrer individuellen Kostenstruktur und den relevanten Produktionsbedingungen orientieren und daher unterschiedliche

\footnotetext{
33 Für weitergehende Betrachtungen kann an dieser Stelle auf die Dissertation von Rüffer (In Vorbereitung) verwiesen werden.
} 
Angebote abgeben werden. So können die Aufträge zur Produktion ökologischer Güter marktanalog auf der Grundlage einzelwirtschaftlich kalkulierter Angebote an die leistungsfähigsten Landwirte vergeben werden. ${ }^{34}$

\subsection{Die Umsetzungsphase}

Die im Januar 2004 begonnene praktische Erprobung des Honorierungssystems erstreckte sich bis zum August 2006. Im Folgenden wird eingangs kurz die Modellregion vorgestellt und anschließend ein Abriss über den Ablauf der Umsetzungsphase gegeben.

\subsubsection{Die Modellregion - Der Landkreis Northeim}

Als Modellregion der praktischen Erprobung fungierte der Landkreis Northeim in Südniedersachsen. Er grenzt unmittelbar an den Landkreis Göttingen und zeichnet sich neben dieser räumlichen Nähe zur Universität Göttingen durch seine vielfältige Landschaftsstruktur, seine landwirtschaftliche Flächennutzung und ein großes Interesse seitens der Agrarverwaltung und den relevanten Interessenvertretern an der Umsetzung des Forschungsprojekts aus.

Der Landkreis Northeim gehört zur naturräumlichen Region „Weser- und Leinebergland“ und ist durch eine hohe geomorphologische Vielfalt (südwestliches Harzvorland, Senken, Solling und Sollingvorland) gekennzeichnet. Diese landschaftliche Vielfalt hat entsprechende Auswirkungen auf die Bewirtschaftung der landwirtschaftlichen Flächen, welche eine extensive Nutzung in Bereichen des Berg- und Hügellandes und eine überwiegend intensive Nutzung in Gebieten mit einem hohen Ertragspotential, wie Talauen größerer Fließgewässer, beinhaltet (Landkreis Northeim 1988, S. 511).

Die Flächengröße des Landkreises Northeim beträgt rund $1.265 \mathrm{~km}^{2}$. Als landwirtschaftliche Nutzfläche werden von den circa 1.400 in der Modellregion angesiedelten landwirtschaftlichen Betrieben insgesamt 57.362 ha bewirtschaftet.

\footnotetext{
34 In der vorliegenden Arbeit wird in Kapitel Fünf ausführlich auf die Grundlagen von Ausschreibungen eingegangen. Die praktische Ausgestaltung und Umsetzung der innerhalb des Forschungsprojekts durchgeführten Ausschreibungsverfahren wird zusammen mit den Ergebnissen in Kapitel Sieben betrachtet.
} 
Die Flächennutzung im Landkreis Northeim gliedert sich in 47.321 ha als Ackerland und 9.864 ha als Dauergrünland bewirtschaftete Fläche (Niedersächsisches Landsamt für Statistik 1999; 2003).

Da der Schutz von Grünlandflächen in der Umsetzungsphase der ergebnisorientierten Honorierung eine zentrale Rolle eingenommen hat, wird die fundamentale praktische Problemstellung in der Modellregion nun kurz an einem Beispiel erläutert.

Die Gefährdung der Biodiversität im Grünland liegt hier vor, da in den eher intensiv genutzten und ertragreichen Gebieten im Landkreis Northeim ein Anreiz zum Grünlandumbruch durch Ackernutzung gegeben ist und in den eher extensiv genutzten Gebieten die Gefahr einer Nutzungsaufgabe mit einer nachfolgenden Sukzession oder Aufforstung vorhanden ist (Bertke et al. 2002, S. 193-194; Bertke et al. 2003c, S. 48). Um dieser ökologisch nicht wünschenswerten Tendenz entgegenzuwirken, ist es notwendig durch eine angemessene Ausgestaltung des Honorierungssystems Anreize zu setzen, die zum Erhalt der Grünlandflächen beitragen und zudem ökonomisch zu rechtfertigen sind.

Bei einer Honorierung ökologischer Leistungen durch eine für die ganze Region einheitlichen Prämie würde sehr wahrscheinlich der Fall eintreten, dass es auch in der Modellregion zu Allokationsverlusten kommt. Zur Überwindung oder zumindest Minimierung dieser Problematik wurde im Landkreis Northeim erstmalig das in dieser Form ausgestaltete Ausschreibungsverfahren zur Honorierung ökologischer Leistungen der Landwirtschaft durchgeführt.

\subsubsection{Ablauf der Umsetzungsphase}

Die beiden dieser Arbeit zugrunde liegenden Ausschreibungsverfahren haben im Zeitraum 2004 bis 2005 und im Jahr 2006 stattgefunden und werden nun jeweils hinsichtlich ihres zeitlichen Ablaufs skizziert.

\section{Ausschreibung 2004/2005}

Zu Beginn des ersten Ausschreibungsverfahrens hat der Regional Beirat Northeim im April 2004 den Nachfragekatalog beschlossen und die Ausschreibung wurde am 01. Juni 2004 öffentlich bekannt gemacht. Parallel dazu wurden drei räumlich über die Modellregion verteilte Informationsveranstaltungen für interessierte Landwirte durchgeführt, bei denen der Ablauf des Ausschreibungsverfahrens und die 
Ausschreibungsunterlagen erläutert wurden. Die Angebotsfrist endete nach sechs Wochen am 10. Juli 2004 und bis zum Ende der Zuschlagsfrist am 17. Juli 2004 wurden nach der Submission der Angebote die entsprechenden Zuschläge und Absagen verschickt. Die Kontrolle der ökologischen Güter erfolgte bis zum 31. Juli 2005 und die Auszahlung an die Landwirte nach einer erfolgreichen Kontrolle im August 2005.

Im Verlauf des Ausschreibungsverfahrens wurden zudem zwei schriftliche Befragungen in der Modellregion durchgeführt, welche die Perspektive der Landwirte erfasst haben und deren Ergebnisse einen maßgeblichen Anteil an dieser Arbeit und der darin vorgenommenen Bewertung des praktischen Einsatzes von Ausschreibungsverfahren und der Diskussion von Transaktionskosten der Landwirte haben.

\section{Ausschreibung 2006}

Das zweite Ausschreibungsverfahren wurde auf der Grundlage des durch den Regionalen Beirat Northeim im Januar 2006 beschlossenen Nachfragekatalogs ökologischer Güter durchgeführt.

Im Verlauf des Ausschreibungsverfahrens hatten die Landwirte bis zur Angebotsfrist am 31. März 2006 Zeit ihre Angebote abzugeben, die seitens der Projektmitarbeiter bis zum 07. April 2006 bewertet wurden. Die Kontrolle der ökologischen Güter erfolgte bedingt durch zeitliche Restriktionen des Forschungsprojekts bereits bis zum 31. Juli desselben Jahres, so dass die die Landwirte im Fall einer positiven Kontrolle im August 2006 ausgezahlt werden konnten.

Darüber hinaus wurde innerhalb des zweiten Ausschreibungsverfahrens eine weitere schriftliche Befragung von Landwirten in der Modellregion durchgeführt, welche die maßgeblichen Aspekte der ersten beiden Befragungen zusammengeführt hat und eine Erweiterung der Datengrundlage unter Einbeziehung eines Vergleichs zweier Befragungsperioden ermöglicht. 
Einen zusammenfassenden Überblick über die Struktur des Honorierungssystems und den Ablauf der Ausschreibungsverfahren in der Umsetzungsphase 2004 bis 2006 im Landkreis Northeim gibt Abbildung 3-1. ${ }^{35}$

Abb. 3-1: Struktur des Honorierungssystems in der Umsetzungsphase

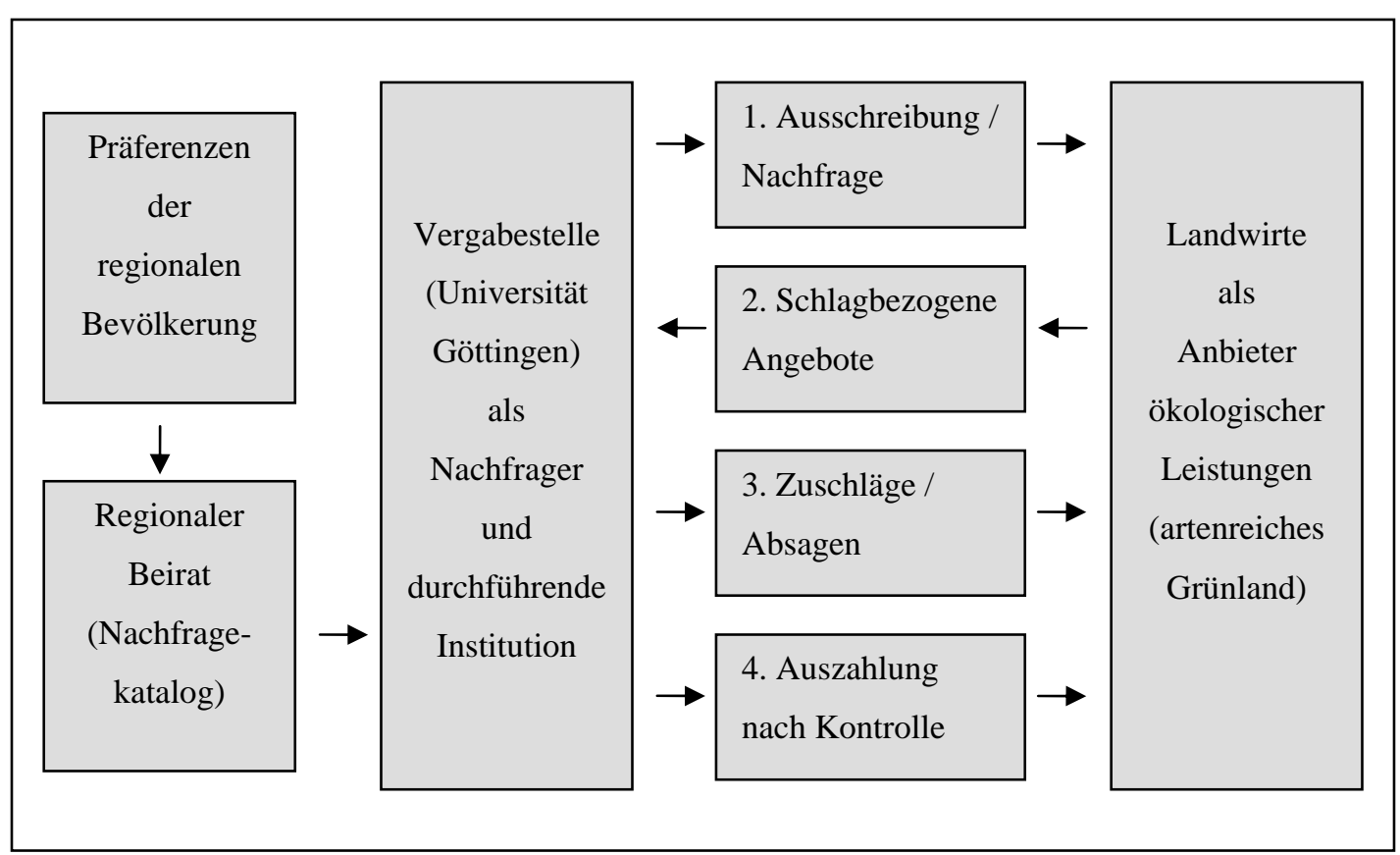

Quelle: Eigene Darstellung.

Der Ablauf des Ausschreibungsverfahrens lässt sich demzufolge so umreißen, dass der Regionale Beirat Northeim zu Beginn unter Einbeziehung regionaler Bevölkerungspräferenzen einen Nachfragekatalog ökologischer Güter beschließt. Auf der Grundlage dieses Nachfragekatalogs wird das Ausschreibungsverfahren seitens der Vergabestelle (hier der Universität Göttingen) durchgeführt, wobei die Vergabestelle als Nachfrager und die Landwirte als Anbieter ökologischer Leistungen auftreten. Nach der öffentlichen Bekanntmachung der Ausschreibung können die teilnahmeberechtigten Landwirte schlagbezogene Angebote abgeben, welche seitens der Vergabestelle nach der Angebotsfrist bewertet werden. Eine Auszahlung an die Landwirte erfolgt, nachdem die vertragkonforme Leistungserbringung auf den Grünlandflächen nachgewiesen werden konnte.

\footnotetext{
${ }^{35}$ Weitere im Rahmen der Umsetzungsphase im Landkreis Northeim in anderen Arbeitsbereichen durchgeführte und hier nicht näher betrachtete Arbeiten umfassen die Ermittlung von regionalen Bevölkerungspräferenzen und ihre Einbindung in die Beiratsentscheidung, die Kartierung von Grünlandflächen und die Kontrolle der ökologischen Güter sowie die Entwicklung von Indikatoren floristischer Artenvielfalt.
} 


\section{Transaktionskostenökonomik}

Die Transaktionskostenökonomik hat ihren Ursprung in den 30er Jahren des vergangenen Jahrhunderts, wobei als Ausgangspunkt in der Regel die grundlegende Erkenntnis von Coase gesehen wird, dass die Koordination ökonomischer Aktivitäten über Märkte nicht kostenlos vonstatten geht (Coase 1937, S. 387-394). Diese Kosten können so hoch sein, dass der Markt als Koordinationsmechanismus versagt und andere Formen der Abwicklung von Transaktionen gewählt werden. Coase begründet damit die Entstehung von Unternehmen, in denen eine entsprechende Transaktion zu geringeren Kosten als über Märkte erfolgen kann (Coase 1937, S. 404-405).

Im Anschluss an die fundamental durch die Herangehensweise von Coase geprägten Arbeiten wurde die Transaktionskostenökonomik maßgeblich von Williamson weiterentwickelt und generalisiert. Somit wird nunmehr der Anspruch erhoben, nicht ausschließlich die Alternativen Markt und Unternehmen, sondern sämtliche Koordinations- bzw. Vertragsformen unter dem Aspekt der Transaktionskosten untersuchen zu können (Williamson 1990a, S. 17-20).

Vor dem Hintergrund des in der vorliegenden Arbeit verfolgten Ansatzes der praktischen Erprobung von Ausschreibungen für Umweltleistungen ist folglich als Ziel der Transaktionskostenökonomik vor allem das Effizienzziel zu nennen (Williamson 1990a, S. 19 und S. 30-34). Ebenso wie in der Agrarumweltpolitik durch den Einsatz eines Ausschreibungsverfahrens anstelle einer Einheitsprämie die im Zuge dessen zu erwartenden Effizienzsteigerungen des öffentlichen Mitteleinsatzes ein entscheidendes Ziel darstellen, betrachtet die Transaktionskostenökonomik Transaktionen und Vertragsbeziehungen im Hinblick auf transaktionskostenmindernde und effizienzsteigernde Effekte.

Zu Beginn des Kapitels wird in Abschnitt 4.1 in Form eines Exkurses auf die Entwicklung der Transaktionskostenökonomik von ihren dogmenhistorischen Vorläufern bis hin zu den fundamentalen Arbeiten von Williamson eingegangen. Im Anschluss gibt Abschnitt 4.2 erste Definitionen zentraler transaktionskostenökonomischer Begriffe und thematisiert damit verbundene Abgrenzungsprobleme. 
Mit der Einführung der menschlichen Verhaltensannahmen beginnt in Abschnitt 4.3 die Darstellung des Analyserahmens der Transaktionskostenökonomik. Dabei wird der Einfluss dieser vom traditionellen ökonomischen Menschenbild abweichenden und als realitätsnäher angesehenen Annahmen auf die Durchführung von Transaktionen aufgezeigt. Als zweiter wesentlicher Aspekt wird in Abschnitt 4.4 der Einfluss der Dimensionen auf das Zustandekommen und die Effizienz von Transaktionsbeziehungen betrachtet. Zudem erfolgt ein kurzer Exkurs, der das Konzept der fundamentalen Transformation thematisiert. Den Analyserahmen abschließend führt Abschnitt 4.5 die grundlegenden Beherrschungsstrukturen ein, die in der Regel je nach Ausprägung der Verhaltensannahmen und der Dimensionen zur institutionellen Ausgestaltung von Transaktionsbeziehungen herangezogen werden.

Nachdem das konzeptionelle Gerüst eingeführt ist, wendet sich Abschnitt 4.6 weitergehenden Abgrenzungen von Transaktionskosten zu. Die in der Literatur vorherrschende und maßgeblich von Soziologen und Ökonomen geäußerte Kritik am Ansatz der Transaktionskostenökonomik wird in Abschnitt 4.7 skizziert.

Neben der Transaktionskostenökonomik sind insbesondere die Principal-AgentTheorie $^{36}$ und die Property-Rights-Theorie ${ }^{37}$ sowie darüber hinaus die Neue Politische Ökonomie ${ }^{38}$ und die Verfassungsökonomik ${ }^{39}$ unter dem Forschungszweig

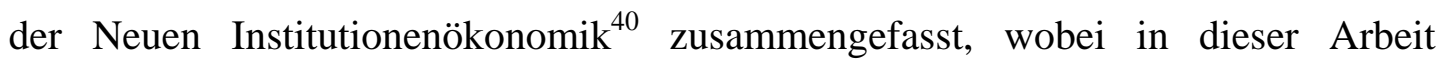
ausschließlich der Ansatz der Transaktionskostenökonomik herangezogen wird.

\footnotetext{
${ }^{36}$ Einen Überblick über die Principal-Agent-Theorie geben Pfaff und Zweifel (1998). Hinsichtlich der Entwicklung des Ansatzes sind vor allem die Arbeiten von Mises (1922); Hayek (1935a; 1935b; 1945); Commons (1934); Barnard (1938); March und Simon (1958); Jensen und Meckling (1976); Holmstom (1979) und Arrow (1985) zu nennen.

${ }^{37}$ Die Entwicklung der Property-Rights-Theorie erfolgte vor allem durch Demsetz (1967); Alchian und Demsetz (1972); Pejovich (1971); Furubotn und Pejovich (1972; 1974); North und Thomas (1973) und North (1988; 1990).

${ }^{38}$ Die Neue Politische Ökonomie wurde von Downs (1968) geprägt. Siehe diesbezüglich auch Kirchgässner (1991) und Richter (1994).

${ }_{39}$ Einen Überblick über die Verfassungsökonomik geben beispielsweise Lee und McKenzie (1987); Buchanan (1991) und Brennan und Buchanan (1993).

${ }^{40}$ Für einen Überblick über den Gesamtkontext der Neuen Institutionenökonomik siehe exemplarisch Thiele (1994); Feldmann (1995); Cezanne und Mayer (1998); Voigt (2002) und Richter und Furubotn (2003).
} 


\subsection{Exkurs: Entwicklung der Transaktionskostenökonomik}

Wie eingangs angedeutet wurde, stellt „The Nature of the Firm“ von Coase aus dem Jahr 1937 traditionell den Ausgangspunkt der Transaktionskostenökonomik dar. Dies muss als eine ungenaue Interpretation angesehen werden, denn die Idee der Analyse und Relevanz von Transaktionen und den mit ihnen verbundenen Kosten wurde implizit schon von Smith im Jahr 1776 (hier 1776/1904) sowie von Mill (1848) als auch von Marshall (1890) aufgegriffen.

Um eine Einordnung der Transaktionskostenökonomik vorzunehmen und ihre Tradition in der Ökonomik zu beleuchten, erfolgt zunächst als Exkurs eine Übersicht über einige für die Entwicklung des Ansatzes maßgebliche Arbeiten. ${ }^{41}$

Die erste im engeren Sinn der Entwicklung der Transaktionskostenökonomik zuzurechnende Arbeit ist „Risk, Uncertainty, and Profits“ von Knight (1922).

Knight betrachtet Unternehmen als Transaktionsstrukturen und weist neben der Zweiseitigkeit von Transaktionen darauf hin, dass es entscheidend ist, im Rahmen der Analyse dieser Transaktionsbeziehungen das tatsächliche menschliche Verhalten zu untersuchen. Dies ist geprägt von Misstrauen und der Gefahr, dass es bei der Zusicherung und Durchführung von Transaktionen $\mathrm{zu}$ einer nicht vereinbarungskonformen Erfüllung kommt, da für die Transaktionspartner Anreize bestehen, diese Situation zum eigenen Vorteil auszunutzen (Knight 1922, S. 270). Im Zuge dessen stellt die von Knight als moralisches Risiko bezeichnete Annahme über menschliches Verhalten jenen entscheidenden Aspekt dar, den es innerhalb von Transaktionsbeziehungen zu überwinden gilt (Knight 1922, S. 260).

Hinsichtlich der in die Transaktionskostenökonomik einbezogenen rechtswissenschaftlichen Literatur stellt die Arbeit „What Price Contract? An Essay in Perspective“ (Llewellyn 1931) einen grundlegenden Beitrag dar.

Llewellyn setzt sich darin mit der Bedeutung von Verträgen für die Ordnung der Gesellschaft auseinander und kritisiert die klassische Vertragstheorie für die Vernachlässigung der zu erzielenden Vertragszwecke und die Schwerpunktsetzung

\footnotetext{
${ }^{41}$ Die Darstellung ist zwangsläufig nicht vollständig und beleuchtet einige ausgewählte Arbeiten, in denen erstmals die jeweiligen fundamentalen Aspekte der Transaktionskostenökonomik aufgezeigt wurden.
} 
auf formale Einzelheiten. Eine derartige Fokussierung auf Ausgestaltungsfragen könne dazu führen, dass die eigentliche Beantwortung einer sachlichen Fragestellung verzögert wird (Llewellyn 1931, S. 728). Zur Lösung entwickelt Llewellyn seine Vorstellung von Verträgen als rechtliche Rahmenbedingungen und unterscheidet zwischen eisernen und nachgiebigen Regeln (Llewellyn 1931, S 729). Die Bedeutung eines Vertrags sollte demzufolge hauptsächlich darin liegen, dass ein rechtlicher Rahmen geschaffen wird, der die Beziehungen zwischen handelnden Menschen möglichst umfassend regeln und in Zweifelsfällen eine juristische Richtlinie vorgeben kann. Erst für den Fall, dass es in Einzelfällen einer darüber hinausgehenden Klärung bedarf, ist vorgesehen dass Gerichte als entscheidende Instanzen eingreifen (Llewellyn 1931, S. 736-737).

Maßgebliche wirtschaftstheoretische Arbeiten zur Berücksichtigung von Transaktionen wurden von Commons sowohl in dem Aufsatz „Institutional Economics“ (1931) als auch ausführlich in der darauf aufbauenden Arbeit „Institutional Economics. It’s Place in Political Economy“ (1934) vorgenommen. Hierbei stellt Commons Transaktionen in den Mittelpunkt seiner Betrachtungen und erhebt sie zur Basiseinheit der Institutionenanalyse (Commons 1931, S. 652; Commons 1934, S. 4-8). ${ }^{42}$ Transaktionen werden als kleinste Einheit der Institutionenökonomik angesehen und im Zuge dessen wird eine stärkere mikroökonomische Analyse von Tauschbeziehungen vertreten: „[...] the smallest unit of the institutional economists is a unit of activity - a transaction, with its participants“ (Commons 1931, S. 652). Commons sieht institutionelle Regelungen ebenfalls als eine Möglichkeit zur Vermeidung oder zumindest Minimierung von Konflikten zwischen Individuen an (Commons 1934, S. 6).

In dem bereits angesprochenen Aufsatz „The Nature of the Firm“ beschäftigt sich Coase mit der Frage, warum in einer Marktwirtschaft Unternehmen existieren und wirtschaftliche Aktivitäten nicht ausschließlich über Märkte, sondern auch innerhalb von Unternehmen abgewickelt werden.

Die Existenz von Unternehmen begründet Coase dadurch, dass mit der Nutzung von Märkten Kosten verbunden sind und die Institution der Unternehmung dazu beitragen kann, diese „costs of using the price mechanism“ (Coase 1937, S. 390)

\footnotetext{
${ }^{42}$ Siehe hierzu ausführlich Kapitel 1, Abschnitt 2: From Exchange to Transactions (Commons 1934).
} 
einzusparen. ${ }^{43}$ Jedoch ist auch die unternehmensinterne Koordination mit Kosten verbunden, womit Märkte und Unternehmen grundsätzlich unterschiedliche Koordinationsmechanismen darstellen (Coase 1937, S. 404-405). ${ }^{44}$

Barnard untersucht in seiner der Organisationstheorie zuzuordnenden Arbeit „The Functions of the Executive“ (1938) unter anderem Organisationen und ihre Anpassung an sich im Wandel befindliche Umgebungen (Barnard 1938, S. 4).

Dabei betont Barnard die durch physische, biologische und soziale Faktoren beeinflussten Schranken menschlicher Rationalität und folgert, dass sämtliche Organisationen in der Realität nicht vollkommen rational ausgestaltet werden können (Barnard 1938, S. 12-45). Vielmehr sind hinsichtlich des fortgesetzten Wandels von Organisationen, vor dem Hintergrund einer lediglich intendierten Rationalität menschlichen Handelns und der damit verbundenen hohen Komplexität von Entscheidungssituationen, adaptive Entscheidungsprozesse unter Einbeziehung von implizitem Wissen notwendig, welche sich an den jeweiligen institutionellen Gegebenheiten orientieren (Barnard 1938, S. 291).

Als herausragender Vertreter der Österreichischen Schule untersucht Hayek nicht nur die Entstehung und Funktion von sozialen Institutionen, sondern auch ihre Verwurzelung in der menschlichen Psyche (Feldmann 1995, S. 36). Im Zuge dessen betont Hayek die institutionelle Abhängigkeit von Ordnungen und diskutiert im Hinblick einer kritischen Auseinandersetzung mit den praktischen und theoretischen Versuchen sozialistischer Planung während der 30er Jahre des vergangenen Jahrhunderts die Grenzen einer sozialistischen Planung und die Vorzüge kapitalistischen Wirtschaftens. Konträr zur herrschenden wirtschaftstheoretischen Lehre sieht Hayek die zentralen ökonomischen Fragestellungen in schnellen Anpassungsprozessen sowie der Wissensteilung und betont die Notwendigkeit der Analyse adaptiver Systeme unter Einbeziehung individuellen Wissens (Hayek 1945, S. 523-524).

Speziell akzentuiert Hayek die Bedeutung von begrenzter Rationalität, Unsicherheit und individuellem Wissen in dem hier relevanten Zusammenhang in seiner Arbeit „The Use of Knowledge in Society“ (1945). Dabei konzentriert sich Hayek

\footnotetext{
${ }^{43}$ Der Begriff der Transaktionskosten wurde von Coase noch nicht verwendet.

${ }^{44}$ Der Ansatz von Coase steht insofern im Gegensatz zur neoklassischen Sichtweise, da er Märkte und Unternehmen als grundsätzlich (begrenzt) substitutierbare Koordinationsmechanismen ansieht.
} 
traditionell auf die Rolle des Marktes, der als Institution zur Bildung von Preisen führt, welche entsprechende Informationen übermitteln und dadurch individuelles Handeln bei begrenzter Rationalität und Unsicherheit koordinieren (Hayek 1945, S. 523)..$^{45}$

Seit den 50er Jahren des vergangenen Jahrhunderts führt Simon beginnend mit „Models of Man: Social and Rational“ (1957) die Rationalitätsanalyse von Barnard weiter, befasst sich ausführlich mit Fragen der Entscheidungsfindung in Unternehmen unter Unsicherheit und entwickelt im Gegensatz zum Ansatz des rational handelnden homo oeconomicus sukzessive das Konzept der begrenzten Rationalität. $^{46}$

Demnach verfügen Menschen lediglich über eingeschränkte Fähigkeiten zur Informationsverarbeitung und unterliegen systematischen Fehleinschätzungen bezüglich der vorhandenen Informationen, der eigenen kognitiven Fähigkeiten und den Ergebnissen des Entscheidungsfindungsprozesses (Simon 1957, S. 198). Menschen handeln folglich nur der Intention nach rational, was zu eingeschränkt rationalen Ergebnissen ihres Handelns führt (Simon 1961, S. xxiv). Darauf aufbauend entwickelt Simon die Hypothese des satisfizierenden Verhaltens, wonach Menschen bedingt durch die oben geschilderte beschränkte Rationalität Entscheidungen nicht als Maximierer, sondern als Satisfizierer treffen. Es werden also realistische Ziele gesetzt, bei deren Erreichen eine Bedürfnisbefriedigung eintritt; im Fall eines Nichterreichens wird entweder das Zielniveau oder die Entscheidung angepasst (Simon 1957, S. 204-205).

In einer scheinbaren Kehrtwendung zu „The Nature of the Firm“ fokussiert Coase in „The Problem of Social Cost“ (1960) eine imaginäre Welt ohne Transaktionskosten, in der jeder über alles sofort und vollkommen informiert ist.

Dies besagt, dass unabhängig von der ursprünglichen Ausstattung an Verfügungsrechten das Externalitäten-Problem durch die privaten Akteure im Zuge von Verhandlungen gelöst werden kann und ein pareto-effizientes Ergebnis ${ }^{47}$ erzielt

\footnotetext{
${ }^{45}$ Die in jener Zeit vorherrschende Gleichgewichtsbetrachtung innerhalb der Wirtschaftstheorie wurde von Hayek lediglich als Vorübung zur Analyse der tatsächlich relevanten Fragestellungen angesehen (Hayek 1945, S. 530).

${ }^{46}$ Siehe hierzu diese in Form einer Aufsatzsammlung grundlegende Arbeit von Simon (1957).

${ }^{47}$ Entsprechende Wohlfahrtseffekte sind nicht Teil der Analyse und Coase beschränkt sich bewusst auf die Frage einer effizienten Ressourcenallokation.
} 
wird: „[In] a world without transaction costs, it does not matter what the law is, since people can always negotiate without cost to acquire, subdivide and combine rights whenever this would increase the value of production." (Coase 1960, S. 14). Coase zeigt, dass es in der zugrunde gelegten Welt keine pareto-relevanten externen Effekte geben kann, da sich die Akteure angesichts fehlender Transaktionskosten spontan und augenblicklich auf einen pareto-optimalen Zustand verständigen werden. ${ }^{48}$ Daraus kann gefolgert werden, dass tatsächlich beobachtete Externalitäten in der Existenz von Transaktionskosten begründet sind und die Betrachtung von externen Effekten eine detaillierte Untersuchung des Einflusses von Transaktionskosten auf eine spontane Internalisierung erfordert. Die Arbeit von Coase muss demzufolge so interpretiert werden, dass sie deutlich macht, wie grundlegend der tatsächliche Einfluss von Transaktionskosten, und damit verbunden der maßgeblichen Institutionen, im hier betrachteten Kontext ist: „Economic policy involves a choice among alternative social institutions, and these are created by the law or are depent on it.“ (Coase 1960, S. 28). Somit lässt sich Marktversagen durch diesen Aufsatz nunmehr auf Transaktionskosten zurückführen.

Der Bedeutung der mit einer Informationsbeschaffung verbundenen Kosten hat sich Stigler mit seinem Aufsatz „The Economics of Information“ (1961) gewidmet und Transaktionskosten weitestgehend mit Such- und Informationskosten gleichgesetzt (Stigler 1961, S. 213-216).

Im Zuge dessen untersucht Stigler den Einfluss der Suche und Bewertung potentieller Vertragspartners hinsichtlich der von ihnen angebotenen Gütern und deren Preisen auf die ökonomischen Aktivitäten von Wirtschaftssubjekten. Stigler kommt zu dem Schluss, dass Akteure so lange Such- und Informationsgewinnungsaktivitäten anstellen werden, bis der erwartete Grenznutzen den damit verbundenen Grenzkosten entspricht (Stigler 1961, S. 216).

\footnotetext{
48 Das nur in dieser imaginären Welt gültige „Coase-Theorem“ (wobei Coase selbst den Begriff „Coase-Theorem“ nicht verwendet hat) ging insbesondere in die deutschsprachige umweltökonomische Literatur als Verhandlungslösung ein (exemplarisch Siebert 1978, S. 90-95; Weimann 1995, S. 38-59; Fees 1995, S. 99-113). Es wurde gefolgert, dass staatliche Eingriffe zur Lösung des Externalitäten-Problems nicht notwendig sind und entsprechende Institutionen irrelevant seien. Vermeintliche Kritiker des Coase-Theorems wiesen darauf hin, dass eine derartige Verhandlungslösung in der Realität angesichts hoher Transaktionskosten in vielen Fällen nicht zustande kommen werde. Zudem wurde Coase Inkonsistenz dahin gehend vorgeworfen, dass er - im krassen Gegensatz zu seiner 1937er Arbeit - Transaktionskosten nun gänzlich ignorieren würde (Streissler 1993, S. 90-93). Coase hatte jedoch das genaue Gegenteil von dem bewiesen, was weithin als Verhandlungslösung bekannt geworden ist.
} 
Der Argumentation von Stigler folgend, betrachtet Alchian in „Information Costs, Pricing and Resource Unemployment“ (1969) die im Rahmen der Beschaffung und Verarbeitung von Informationen bei Marktaktivitäten auftretenden Informationskosten, welche implizit als Transaktionskosten angesehen werden (Alchian 1969, S. 109-111).

Im Anschluss an „The Problem of Social Costs“ und der dortigen Betonung des Zusammenhangs von Marktversagen und Transaktionskosten entstand „The Organisation of Economic Activity: Issues Pertinent to the Choice of Market versus Non-Market Allocation” von Arrow (1969).

Arrow behandelt die Frage des Marktversagens im Bereich der Theorie der öffentlichen Güter und betont, dass Marktversagen nicht als etwas Absolutes aufgefasst werden kann und es zielführender ist, eine allgemeine Kategorie wie Transaktionskosten zu betrachten, welche als „costs of running the economic system“ (Arrow 1969, S. 48) bezeichnet werden. Für Arrow sind diese Betriebskosten des Wirtschaftssystems die entscheidende Größe für die Einschränkung oder Verhinderung der Entstehung von Märkten (Arrow 1969, S. 48). Die Identifizierung des Einflusses von Transaktionskosten sollte folglich innerhalb der Bewertung verschiedener Allokationsmechanismen und Vertragsformen unter Berücksichtigung kulturspezifischer Einflussfaktoren eine zentrale Rolle einnehmen (Arrow 1969, S. 63).

In seinem Aufsatz „The Vertical Integration of Production: Market Failure Considerations“ betrachtet Williamson (1971) ebenfalls den Zusammenhang zwischen Marktversagen und Transaktionskosten.

Williamson bezieht sich eingangs auf die oben angesprochenen Arbeiten von Coase und Arrow, betrachtet dann Marktversagen jedoch nur eingeschränkt als Versagen, da das Vorhandensein und die Höhe von Transaktionskosten dazu führen können, dass Markttransaktionen durch unternehmensinterne Organisationen substituiert werden: „What are refferd to here as market failures are failures only in the limited sense that they involve transaction costs that can be attenuated by substituting internal organization for market exchange.“ (Williamson 1971, S. 114). Dies führt mit dem Problem der vertikalen Integration zu einer auch für weitere Arbeiten von 
Williamson zentralen Fragestellung; der unternehmerischen Make-or-buyEntscheidung (Williamson 1971, S. 27-28).

Einsichtig ist, dass ceteris paribus die relativen Nachteile marktlicher Verträge als die relativen Vorteile der unternehmensinternen Abwicklung betrachtet werden können. Auch im Rückgriff auf bestehende Arbeiten kommt Williamson zu dem Schluss, dass es unmöglich ist bzw. prohibitiv hohe Kosten verursachen würde, alle Eventualitäten vorauszusehen und ex-ante in Verträgen zu berücksichtigen. Daher sind Verträge in der Praxis immer unvollständig und stellen in Kombination mit opportunistischem Verhalten das zentrale, durch die Wahl einer geeigneten Beherrschungsstruktur zu lösende, Problem dar (Williamson 1971, S. 27-28).

Die zweite in diesem Zusammenhang maßgebliche Arbeit von Williamson ist „Markets and Hierachies“ (1975).

Hier wird das Ziel verfolgt, durch die Verbindung von Verhaltensannahmen und spezifischer Umwelteigenschaften auf Probleme marktlicher Verträge hinzuweisen und aufzuzeigen, wann eine Transaktion relativ besser unternehmensintern abgewickelt werden kann.

Das zugrunde gelegte Menschenbild ist definiert durch die für die gesamte Transaktionskostenökonomik zentralen Annahmen begrenzte Rationalität und Opportunismus (Williamson 1975, S. 20-30). Als Umwelteigenschaften führt Williamson Unsicherheit und Abhängigkeit ein und untersucht einerseits die Kombination von begrenzter Rationalität mit Unsicherheit und andererseits die Kombination von Opportunismus und Abhängigkeit hinsichtlich der damit verbundenen vertraglichen Transaktionsprobleme auf Märkten (Williamson 1975, S. 20-40). Zusammenfassend hat Williamson Faktoren identifiziert, von denen Transaktionsprobleme ausgehen und die eine institutionelle Lösung erfordern. In diesem Kontext erweisen sich Unternehmen bzw. Hierarchien unter bestimmten Bedingungen gegenüber marktlichen Verträgen als vorteilhaft, was ihre Existenz und ihr Wachstum durch vertikale Integration erklären kann. ${ }^{49}$

\footnotetext{
49 Darüber hinaus führt Williamson in dieser Arbeit auch erstmals den Begriff der Neuen Institutionenökonomik ein: „A broad based interest among economists in what might be referred to as the „new institutional economics“ has developt in recent years.“ (Williamson 1975, S. 1).
} 
Die zentrale transaktionskostenökonomische Arbeit von Williamson stellt „The Economic Institutions of Capitalism“ (1985) beziehungsweise in ihrer deutschen Übersetzung „Die ökonomischen Institutionen des Kapitalismus“ (1990a) ${ }^{50}$ dar. Williamson verdichtet sein Konzept auf das Zusammenspiel der zentralen Faktoren der Analyse von Transaktionsbeziehungen, welche im Rahmen dieser Arbeit in den folgenden Abschnitten ausführlich betrachtet werden.

Im Anschluss hat Williamson weitere Arbeiten veröffentlicht, die den Analyserahmen der Transaktionskostenökonomik hinsichtlich verschiedener Teilaspekte erweitert haben und auf die in der vorliegenden Arbeit nicht explizit eingegangen wird (Williamson 1993; 1996; 1998; 2000).

Es ist deutlich geworden, dass die Entwicklung der Transaktionskostenökonomik ihren Anfang maßgeblich in den 30er-Jahren des vergangenen Jahrhunderts hatte und durch verschiedenste der Wirtschafts-, der Organisations- und auch der Rechtstheorie zuzuordnende Arbeiten geprägt wurde. Im Zuge dieser ersten Ansätze wurden bereits wesentliche Erkenntnisse für die Untersuchung ökonomischer Institutionen und Transaktionen zugänglich gemacht.

Von Knight wurde die Annahme des Opportunismus zur Erklärung menschlichen Verhaltens als eine allgemein verbreitete und subtil auftretende Merkmalsausprägung eingeführt. Commons erhob die Transaktion zur Basiseinheit der ökonomischen Analyse und im Zuge dessen wurde sowohl von Commons als auch von Barnard die Ausgestaltung von Tauschbeziehungen als die Hauptaufgabe ökonomischer Institutionen betrachtet. Die Untersuchung von Verträgen als Gegenstück zu ökonomischen Organisationen wurde von Llewellyn angestoßen. Auf Coase geht die Überwindung der getrennten Untersuchung der Marktorganisation und der internen Unternehmensorganisation zurück. Die Bedeutung der begrenzten Rationalität menschlicher Entscheidungsprozesse wurde wegweisend von Simon und von Hayek betont.

Auf der Grundlage dieser als entscheidende Eckpfeiler anzusehenden Arbeiten entwickelte sich die Transaktionskostenökonomik sukzessive fort. ${ }^{51}$

\footnotetext{
${ }^{50}$ Im Übrigen ist dies die einzige ins Deutsche übersetzte Arbeit von Williamson.

51 Für einen Überblick siehe Williamson und Masten (1999); Allen (2000); Börner und Macher (2001); Richter und Furubotn (2003) und James (2006).
} 
Für die nachfolgenden Ausführungen wird insbesondere auf die ersten drei Kapitel und die zusammenfassenden Überlegungen aus „Die ökonomischen Institutionen des Kapitalismus“ (1990a) als übersetzte Ausgabe der grundlegenden Arbeit von Williamson eingegangen.

\subsection{Grundlegende Definitionen und begriffliche Abgrenzungen}

Im Anschluss an die Entwicklung der Transaktionskostenökonomik und vor der detaillierten Betrachtung ihrer konzeptionellen Bestandteile werden im Folgenden grundlegende Definitionen und begriffliche Abgrenzungen zu Transaktionen und Transaktionskosten vorgenommen.

\subsubsection{Transaktion}

Eine Transaktion stellt die Basiseinheit der Transaktionskostenökonomik dar (Dugger 1983, S. 95; Dow 1987, S. 14; Picot und Dietl 1990, S. 178; Williamson 1990a, S. 20). Dabei bezieht sich Williamson maßgeblich auf Commons, der erstmalig explizit den Begriff der Transaktion in die ökonomische Analyse eingeführt hat und die Transaktion als „ultimate unit of activity“ (Commons 1931, S. 652) ansieht (Williamson 1990a, S. 3).

Dieser Tradition folgend, können Transaktionen ganz allgemein als „elementare Untersuchungseinheit sozioökonomischer Aktivitäten“ (Picot und Dietl 1990, S. 178) betrachtet werden, wobei mit der Übertragung eines Guts oder einer Leistung einerseits und der Übertragung von Verfügungsrechten andererseits zwei grundsätzliche Arten von Transaktionen zu unterscheiden sind (Richter und Furubotn 2003, S. 55-57).

Die ursprüngliche Sichtweise von Transaktionen als vertraglicher Austausch von Verfügungsrechten wurde von Commons selbst zwischenzeitlich angepasst, so dass sich letztlich folgende Definition von Transaktionen herausgebildet hat: „Transactions [...] are not the exchange of commodities, in the physical sense of delivery, they are the alienation and acquisition, between individuals, of the rights of future ownership of physical things“ (Commons 1934, S. 58). 
Diese Sichtweise betont zudem die soziale Beziehung der Individuen und ihr zielgerichtetes Handeln, welches zeitlich in der Regel vor dem physischen Tausch von Gütern oder Leistungen stattfindet.

Der Austausch von Gütern und Leistungen als zweite Abgrenzung einer Transaktion wird von Williamson wie folgt definiert: „Eine Transaktion findet statt, wenn ein Gut oder eine Leistung über eine technisch trennbare Schnittstelle hinweg übertragen wird. Eine Tätigkeitsphase wird beendet; eine andere beginnt.“ (Williamson 1990a, S. 1). Demnach ist eine Transaktion als ein physischer Transfer zu verstehen, der zwei Aktivitäten miteinander verbindet und zu einem institutionell zu lösenden Koordinationsproblem führt (Richter und Furubotn 2003, S. 55-57). ${ }^{52}$

\subsubsection{Transaktionskosten}

Nach der Transaktion gilt es mit den Transaktionskosten den zweiten essentiellen Begriff zu beleuchten. Dabei ist trotz der wesentlichen Stellung der Transaktionskosten innerhalb der Transaktionskostenökonomik bereits an dieser Stelle anzumerken, dass es keine exakte und allgemeingültige Definition von Transaktionskosten gibt (Allen 2000, S. 912; Börner und Macher 2001, S. 5-8; Richter und Furubotn 2003, S. 57-65; Wang 2003, S. 1-2).

Somit wird nachfolgend der Ansatz verfolgt, grundsätzlich aufzuzeigen, was unter Transaktionskosten subsumiert werden kann und welche grundlegenden Abgrenzungen sich bislang herausgebildet haben.

Als erste Umschreibung wird zu Beginn die von Coase gewählte Begriffsbestimmung herangezogen, im Rahmen derer - noch ohne explizit von Transaktionskosten zu sprechen - argumentiert wird, dass durch Unternehmen Kosten der Nutzung des Preismechanismus eingespart werden können: „The main reason why it is profitable to establish a firm would seem to be that there is a cost of using the price mechanism“ (Coase 1937, S. 390).

\footnotetext{
${ }^{52}$ Neben dieser auf die physische Übertragung abzielenden Definition einer Transaktion findet sich bei Williamson ebenfalls eine Anwendung des Transaktionsbegriffs im Zusammenhang mit der Übertragung von Verfügungsrechten (Williamson 1990a, S. 30-34).
} 
Eine weitreichendere Definition von Transaktionskosten liefert Arrow, indem die gesamten im Rahmen der Anbahnung und Abwicklung von Transaktionen innerhalb eines Wirtschaftssystems entstehenden Kosten als „costs of running the economic system“ (Arrow 1969, S. 48) thematisiert werden.

Eine spezifische Abgrenzung von Transaktionskosten mit dem Bezug auf den auch in dieser Arbeit zentralen Such- und Informationskosten geben sowohl Stigler als auch Alchian, indem von „costs of search“ (Stigler 1961, S. 216) oder „search costs“ (Alchian 1969, S. 110) und „costs of information“ (Alchian 1969, S. 111) gesprochen wird.

Dem Ansatz von Arrow folgend, versteht Williamson Transaktionskosten als Betriebskosten oder genauer gesagt als Reibungskosten innerhalb eines Wirtschaftssystems. Williamson stellt in diesem Zusammenhang den Vergleich mit den Reibungsverlusten beim Betrieb einer Maschine an und charakterisiert Transaktionskosten als das ökonomische Gegenstück dazu (Williamson 1990a, S. 1 und S. 21-22).

Im Zuge dessen geht Williamson davon aus, dass ökonomische Institutionen der Reduzierung dieser Transaktionskosten dienen und Einsparungen dadurch realisiert werden können, dass verschiedene Arten von Transaktionen innerhalb unterschiedlicher institutioneller Ausgestaltungen (den so genannten Beherrschungsstrukturen) zu verschieden hohen Transaktionskosten führen (Williamson 1979, S. 246-253; Williamson 1990a, S. 77; Richter und Furubotn 2003, S. 193-196).

In einer engen Abgrenzung können Transaktionskosten demzufolge als die mit der Anbahnung, Implementierung, Durchsetzung, Kontrolle und Anpassung von Vertragsbeziehungen verbundenen Kosten bezeichnet werden, wobei sich Transaktionen zwischen Individuen und im Rahmen von expliziten oder impliziten Verträgen vollziehen (Williamson 1990a, S. 21-25).

Als Beispiele für diese Aufwendungen von Ressourcen und Zeit sind Such- und Informationskosten, Verhandlungs- und Entscheidungskosten, Kosten der Durchsetzung und Überwachung vertraglicher Vereinbarungen und gesetzlicher 
Regelungen zu nennen (Dahlmann 1979, S. 148; Picot 1982, S. 270; Richter und Furubotn 2003, S. 57-65).

Für einige spezifische Fragestellungen ist es zudem notwendig, eine Differenzierung in Ex-ante-Transaktionskosten und Ex-post-Transaktionskosten vorzunehmen. Die Unterscheidung bezieht sich hierbei nicht auf den Zeitpunkt des tatsächlichen physischen Transfers von Gütern oder Dienstleistungen, sondern auf den Zeitpunkt des relevanten Vertragsabschlusses oder ganz allgemein der Implementierung eines Koordinationsmechanismus. Die Erbringung der vertraglich zugesicherten Leistung erfolgt ebenso wie der physische Transfer in der Regel nach dem Vertragsabschluss (Williamson 1990a, S. 22-25).

Als letzte Abgrenzungsmöglichkeit erfolgt auch für Transaktionskosten die Unterscheidung in fixe und variable Kosten. Fixe Transaktionskosten sind unabhängig von der Häufigkeit mit der eine Transaktion durchgeführt wird und sie fallen beispielsweise bei der Implementierung eines Koordinationsmechanismus an. Demgegenüber sind variable Transaktionskosten abhängig von der Häufigkeit der Durchführung einer Transaktion und variieren entsprechend (Falconer und Whitby 1999, S. 12-13; Richter und Furubotn 2003, S. 58).

\subsection{Verhaltensannahmen}

Innerhalb der Transaktionskostenökonomik nimmt das zugrunde gelegte Menschenbild eine bedeutende Rolle ein und speziell Williamson hebt die Relevanz der Verhaltensannahmen für eine ökonomische Analyse hervor (Williamson 1975, S. 20-21; Williamson 1990a, S. 49-50; Williamson 1996, S. 55).

Menschenbilder, die ganz allgemein als „Einstellungen von Menschen gegenüber Menschen“ (Schanz 1988, S. 115) oder „implizite Persönlichkeitstheorien“ (Weinert 1998, S. 672) angesehen werden können, dienen auch im Rahmen ökonomischer Betrachtungen der Komplexitätsreduktion, indem so die Vielfalt menschlicher Verhaltensmuster und Persönlichkeitsausprägungen vereinfacht und einer strukturierten Analyse zugänglich gemacht werden kann.

Das in der traditionellen Ökonomik vorherrschende Menschenbild ist maßgeblich durch das Konstrukt des homo oeconomicus geprägt. Demgegenüber greift die Transaktionskostenökonomik auf die nachfolgend einzuführenden 
Verhaltensannahmen $^{53}$ begrenzte Rationalität und Opportunismus zurück, um dadurch ein realitätsnäheres menschliches Verhalten zu unterstellen (Coase 1984, S. 231; Williamson 1990a, S. 50; Richter und Furubotn 2003, S. 192-193). ${ }^{54}$

\subsubsection{Begrenzte Rationalität}

Hinsichtlich der Rationalität menschlichen Verhaltens unterscheidet Williamson die drei Stufen Maximierung, begrenzte Rationalität und organische Rationalität (Williamson 1990a, S. 50).

Die Maximierung entspricht der neoklassischen Sichtweise und kann als stärkste Form der Rationalität angesehen werden. Die schwächste Ausprägung stellt die organische Rationalität dar, welche in evolutorischen Ansätzen (Alchian 1950; Hirshleifer 1985) und der Österreichischen Schule (Menger 1968; Hayek 1967; Kirzner 1973) Anwendung findet. Beide Ausprägungen erfahren innerhalb der Transaktionskostenökonomik keine Berücksichtigung, sondern es wird mit der begrenzten Rationalität eine zwischen diesen Ausprägungen anzusiedelnde Verhaltensannahme zugrunde gelegt (Williamson 1990a, S. 50-53).

Die mit der wirtschaftswissenschaftlichen Tradition eng verbundene Annahme der Rationalität menschlichen Verhaltens findet sich somit ebenfalls im Rahmen der Transaktionskostenökonomik wieder. Jedoch in einer abgewandelten Form, denn das auf Simon (1957) zurückgehende und von Williamson übernommene Konzept der begrenzten Rationalität ${ }^{55}$ ist gegen die in der traditionellen neoklassischen Mikroökonomik unterstellte vollkommene Rationalität gerichtet, die für individuelle Entscheidungen vollständige Informationen über damit verbundene Ziele, Mittel und Wirkungen unterstellt.

Mit der Annahme der begrenzten Rationalität soll hingegen berücksichtigt werden, dass Akteure rational handeln wollen, sie jedoch sowohl angesichts ihrer eingeschränkten Kapazitäten zur Informationsgewinnung und -verarbeitung als auch einer durch Unsicherheit geprägten Handlungssituation nicht alle Alternativen und die damit verbundenen Konsequenzen exakt abschätzen können (Simon 1957, S.

\footnotetext{
${ }^{53}$ Die Verhaltensannahmen werden teilweise auch als „human factors“ bezeichnet.

54 Des Weiteren wird im Analyserahmen der Transaktionskostenökonomik die Annahme der Risikoneutralität beibehalten, auf welche an dieser Stelle nicht weiter eingegangen wird.

${ }^{55}$ Teilweise in der Literatur auch als eingeschränkte oder beschränkte Rationalität bezeichnet.
} 
196-199). Begrenzt rationales Verhalten wird von Simon selbst wie folgt umschrieben: „Human behavior is intendedly rational, but only limited so“ (Simon 1961, S. 28).

Menschen handeln infolgedessen im Analyserahmen der Transaktionskostenökonomik nur ihrer Intention nach rational, was zu begrenzt rationalen Ergebnissen ihres Handelns führt (Simon 1957, S. 196-198). Dabei ist eine unvollständige Informationsgewinnung insbesondere durch Transaktionskosten in Form der Aufwendung von Zeit und Ressourcen zu begründen, die ab einer gewissen Höhe eine weitere Informationssuche als nicht mehr lohnenswert erscheinen lassen.

Die eingeschränkte Verarbeitung und Berücksichtigung der vorhandenen Informationen ist hingegen auf die begrenzten kognitiven Fähigkeiten von Menschen zurück zu führen. Diese machen es zum einen unmöglich alle Informationen zu verarbeiten und zum anderen führen sie dazu, dass die Informationen nicht objektiv rational, sondern unter dem Einfluss von individuellen Vorprägungen selektiv verarbeitet werden (Williamson 1975, S. 21-22; Williamson 1996, S. 36-37). Begrenzte Rationalität meint jedoch keinesfalls irrationales Verhalten, denn die Akteure handeln vor dem Hintergrund eines begrenzten Informationsstandes noch immer streng rational (Simon 1957, S. 200-201; Simon 1972, S. 161-176). ${ }^{56}$

Mit dem Fokus auf der Ausgestaltung von Verträgen kann nunmehr gefolgert werden, dass bedingt durch die beschränkte Rationalität der handelnden Individuen Verträge notwendigerweise unvollständig sind und es als Konsequenz daraus zu ineffizienten Marktergebnissen kommen kann. Im Angesicht der begrenzten Rationalität menschlichen Verhaltens gilt es zu untersuchen, welche Kosten der Planung, Anpassung und Überwachung von Transaktionen entstehen und welche Beherrschungs- und Überwachungsstrukturen angemessen sind (Williamson 1990a, S. 52; Williamson 1996, S. 36-37).

\footnotetext{
${ }^{56}$ Weshalb mitunter der von Simon eingeführte und mittlerweile etablierte Begriff der begrenzten Rationalität kritisiert werden kann, denn nicht die Rationalität ist begrenzt, sondern die menschlichen Kapazitäten zur Informationsgewinnung und -verarbeitung, was letztlich zu einem begrenzt rationalen Ergebnis führen kann. Die grundlegende Verhaltensannahme sollte demzufolge treffender mit „begrenzter Information“ oder „begrenzter Wahrnehmung“ bezeichnet werden. Innerhalb dieser Arbeit wird jedoch der gängigen Terminologe folgenden die Bezeichnung begrenzte Rationalität beibehalten.
} 


\subsubsection{Opportunismus}

Analog zur Rationalität können hinsichtlich der individuellen Berücksichtigung des Eigeninteresses drei Stufen unterschieden werden, wobei der Transaktionskostenökonomik mit dem Opportunismus die starke Form der Eigennutzverfolgung zugrunde liegt (Williamson 1990a, S. 53).

Daneben unterscheidet Williamson das schlichte Eigeninteresse entsprechend des neoklassischen Ansatzes als halb-starke Form, bei der regelgebunden und bei voller Transparenz gehandelt wird. Die schwache Form der Eigennutzverfolgung ist der Gehorsam, innerhalb dessen das Eigeninteresse zu vernachlässigen ist (Williamson 1990a, S. 56).

Mit der Verhaltensannahme des Opportunismus charakterisiert Williamson die Verfolgung des Eigeninteresses von Wirtschaftssubjekten, wobei die inhaltliche Nähe zum traditionellen ökonomischen Menschenbild dadurch zum Ausdruck kommt, dass Opportunismus eine Möglichkeit des eigeninteressierten Handelns darstellt (Williamson 1975, S. 26; Williamson 1990b, S. 54-56). Während in der von Williamson kritisierten engen Auslegung der traditionellen ökonomischen Vorstellung von individuellem Eigennutzstreben unterstellt wird, dass sich die handelnden Individuen bei der Verfolgung ihrer Ziele an allgemein anerkannte Regeln halten, die vorhandenen Informationen nicht zurückhalten oder verzerren und sich vertragskonform verhalten, berücksichtigt die Annahme des Opportunismus weitere Aspekte (Williamson 1990a, S. 54).

Der Opportunismus geht über den Eigennutz hinaus und Individuen wird sowohl eigennütziges als auch strategisches Verhalten unterstellt, was bedeutet, dass sie ihre Ziele gegebenenfalls bewusst zu Lasten anderer durchzusetzen versuchen. Es wird ihnen in diesem Analyserahmen folglich die Möglichkeit eingeräumt, dass sie ihren Eigennutz unter Zuhilfenahme von List, Lügen, Betrügen, Stehlen, bewusstem Vertragsbruch usw. verfolgen. Der Opportunismus beschränkt sich jedoch nicht auf diese offensichtlichen Formen und berücksichtigt ebenfalls subtilere Ausprägungen der Täuschung, wie die bewusst unvollständige oder gezielt verfälschte Weitergabe von Informationen (Williamson 1990a, S. 54-55). 
Die Bedeutsamkeit von opportunistischem Verhalten ergibt sich durch die davon ausgehenden Beeinflussungen des Zustandekommens, der Abwicklung und der Überwachung von Transaktionen.

Ohne Opportunismus kann generell von einem vertragskonformen Verhalten ausgegangen werden und eine spezifische Absicherung von Transaktionen wäre nicht notwendig. Dabei ist $\mathrm{zu}$ betonen, dass mit der Verhaltensannahme des Opportunismus nicht unterstellt wird, dass sich alle Akteure immer und zu jeder Zeit opportunistisch verhalten, sondern vielmehr berücksichtigt wird, dass Akteure zeitweilig opportunistisch handeln und unterschiedlich vertrauenswürdig sind; dies jedoch in den seltensten Fällen vorab zu erkennen ist. Somit macht es die Ungewissheit in welchem Maß es zu opportunistischem Verhalten kommt notwendig, Transaktionen und Vertragsbeziehungen durch die Wahl einer adäquaten Beherrschungsstruktur ex-ante bestmöglich abzusichern (Williamson 1990a, S. 55).

Bei der Analyse von Transaktionen kann zudem mit Blick auf den Zeitpunkt des Vertragsabschlusses zwischen Ex-ante-Opportunismus und Ex-post-Opportunismus unterschieden werden. Dabei umfasst der Ex-ante-Opportunismus den Zeitraum bis zum Vertragsabschluss beispielsweise in Form der Übermittlung unzutreffender Information hinsichtlich der Leistungsfähigkeit eines Vertragspartners, was zur Folge hat, dass diese Vertragspartei zu positive Vertragskonditionen aushandeln kann. Der Ex-post-Opportunismus behandelt den Zeitraum nach dem Vertragsabschluss und den Fall, dass sich ein Vertragspartner zu Lasten der anderen Vertragspartei nicht vertragskonform verhält (Williamson 1990a, S. 55; Richter und Furubotn 2003, S. 587). ${ }^{57}$

\subsection{Dimensionen von Transaktionen}

Unter Berücksichtigung der Verhaltensannahmen gilt es zu analysieren, wie Transaktionen in der Realität organisiert sind und welche Merkmale identifiziert werden können, die positiven oder negativen Einfluss auf die Abwicklung von Transaktionen haben (Fischer 1994, S. 582-583).

\footnotetext{
57 Hierbei besteht ein enger Bezug zur Principal-Agent-Theorie und den darin untersuchten Informationsasymmetrien.
} 
Williamson betrachtet zur Verdeutlichung eine Maschine, deren einzelne Komponenten im Idealfall reibungslos ineinander greifen (Williamson 1996, S. 12). Ähnlich kann der Aspekt von Transaktionen betrachtet werden, wobei die einzelne Transaktion die Basis der Analyse darstellt und untersucht wird, welcher Einfluss jeweils von den drei Dimensionen ${ }^{58}$ Faktorspezifität, Unsicherheit und Häufigkeit auf ihre Organisation ausgeht (Williamson 1990a, S. 59-69).

\subsubsection{Faktorspezifität}

Mit der Faktorspezifität werden die im Rahmen einer Transaktion verwendeten Faktoren hinsichtlich der mit ihnen verbundenen spezifischen Investitionen beschrieben (Picot und Dietl 1990, S. 179). In diesem Zusammenhang gilt eine Transaktion bzw. eine Vertragsbeziehung mit steigenden durch sie induzierten Quasi-Renten als zunehmend spezifischer. Dabei ist unter einer Quasi-Rente der Wertverlust $\mathrm{zu}$ verstehen, der eintreten würde, wenn das durch eine spezifische Investition geschaffene Sach- oder Humankapital nicht als Teil der angestrebten, sondern einer nächstbesten Transaktion zum Einsatz kommt (Dietl 1991, S. 95; Richter und Furubotn 2003, S. 589).

Die Faktorspezifität stellt sich demzufolge als ein zeitliches Problem dar und es kann bei spezifischen Transaktionen zu einer restriktiven Bindung in Form von Lock-inEffekten an den jeweiligen Vertragspartner kommen. ${ }^{59}$ Diese können soweit führen, dass der Tausch zwischen ursprünglich eigenständigen Akteuren durch einheitliches Eigentum ersetzt wird (Williamson 1971, S. 116; Williamson 1990a, S. 61). In diesem Zusammenhang spricht Williamson von einer wie folgt zu umschreibenden fundamentalen Transformation: „Was zunächst ein Bietprozess mit einer großen Zahl von Teilnehmern war, wird in der Folge effektiv zu einer Situation bilateralen Angebots“ (Williamson 1990a, S. 70). ${ }^{60}$

\footnotetext{
${ }^{58}$ Die Dimensionen von Transaktionen werden auch als „environmental factors“ bezeichnet.

59 Auf die Relevanz der Faktorspezifität machte bereits Marshall (1890) aufmerksam, als er argumentierte, dass durch den Einsatz von spezialisiertem Sach- oder Humankapital Quasi-Renten erwirtschaftet werden können.

${ }^{60}$ Hinsichtlich der grundsätzlichen Bedeutung der dem Konzept der fundamentalen Transformation innewohnenden Argumentation auch für die weitere praktische Analyse von Ausschreibungen für Umweltleistungen wird die fundamentale Transformation als Exkurs in Abschnitt 4.4 .4 erneut aufgegriffen.
} 
Als Folge dieser hohen Bindung von Vertragspartnern können während der Vertragslaufzeit auftretende Probleme oder durch opportunistisches Verhalten entstehende Konflikte nicht mehr durch einen einfachen Wechsel des Transaktionspartners gelöst werden (Dietl 1991, S. 95-96).

Für die Transaktionskostenökonomik kommt der Faktorspezifität ebenso eine sehr hohe Bedeutung zu, wie für weitere Arbeiten, die sich mit Anreizen in Verträgen befassen. Entscheidend ist im Zuge dessen das zeitliche Zusammentreffen von Faktorspezifität, den im vorherigen Abschnitt behandelten Verhaltensannahmen und Unsicherheit; eine Konstellation, die gerade den Analyserahmen der Transaktionskostenökonomik kennzeichnet (Williamson 1990a, S. 64).

\subsubsection{Unsicherheit}

Die Dimension der Unsicherheit führt zu der Frage, welche Beherrschungs- und Überwachungssysteme bestmöglich dazu beitragen können, durch Unsicherheit bedingte Störungen einer Vertragsbeziehung zu mindern und vor dem Hintergrund der getroffenen Verhaltensannahmen die bestmögliche Anpassungsfähigkeit erwarten lassen (Williamson 1990a, S. 65).

Je nach Art und Ausgestaltung einer Transaktion kann in verschiedenen Ausprägungen eine Unsicherheit über zukünftige vertragsrelevante Ereignisse bestehen, was maßgeblichen Einfluss auf die Effizienz von Transaktionen und ihre Absicherung hat. In diesem Kontext erfolgt eine differenzierte Betrachtung der Unsicherheit hinsichtlich ihrer Beeinflussbarkeit durch die Vertragsparteien, so dass zwischen exogenen Risiken und Verhaltensunsicherheiten $\mathrm{zu}$ unterscheiden ist (Williamson 1990a, S. 65-66; Richter 1994, S. 18-19).

Unter exogenen Risiken sind unerwartete Entwicklungen zu verstehen, die von keiner Vertragspartei im Verlauf der Vertragsdauer beeinflusst werden können und die zum Zeitpunkt des Vertragsabschlusses nicht absehbar sind. ${ }^{61}$ Die im Rahmen der Transaktionskostenökonomik weitaus relevantere Ausprägung ist die auf mögliches opportunistisches Verhalten des Transaktionspartners zurückzuführende

\footnotetext{
${ }^{61} \mathrm{Zu}$ nennen sind hier Naturkatastrophen, andere Fälle von höherer Gewalt oder auch der Tod eines Vertragspartners.
} 
Verhaltensunsicherheit, bei der zwischen Verhaltensunsicherheit vor und nach dem Vertragsabschluss zu unterscheiden ist.

Im Rückgriff auf die Dimension der Faktorspezifität wird deutlich, dass Unsicherheit für unspezifische Transaktionen kein essentielles Problem darstellt, da sich aus der Unsicherheit ergebende Anpassungen mit relativ geringen Kosten verbunden sind. Im Gegensatz dazu kommt der Implementierung von Anpassungsmechanismen und der entsprechenden Ausgestaltung von Beherrschungsstrukturen bei einer hohen Faktorspezifität eine entscheidende Bedeutung zu (Williamson 1990a, S. 68).

Wird bei der Analyse von Transaktionen eine so große Unsicherheit unterstellt, dass sie die Rationalitätsgrenzen der Transaktionspartner übersteigt, kann sie als gegeben betrachtet und für eine explizite Analyse vernachlässigt werden. Die Betrachtung erfolgt dann beschränkt auf die Dimensionen Faktorspezifität und Häufigkeit einer Transaktion (Williamson 1990b, S. 81).

\subsubsection{Häufigkeit}

Als dritte Dimension wird die Häufigkeit einbezogen, die sich mit dem Einfluss von einmalig, gelegentlich oder regelmäßig durchgeführten Transaktionen auf die mit entsprechenden Beherrschungsstrukturen verbundenen Kosten beschäftigt.

Die grundlegende Annahme ist im Zuge dessen, dass spezifische Beherrschungs- und Überwachungssysteme auf die jeweiligen Kontrollnotwendigkeiten exakter eingestellt sind als nicht-spezifische und gleichzeitig zu höheren Transaktionskosten führen (Williamson 1990a, S. 69).

Dabei wird deutlich, dass die Kosten von Absicherungsmechanismen in Relation zu den durchgeführten Transaktionen stehen und sich amortisieren müssen. Dies führt dazu, dass aufwendige und kostenintensive Beherrschungsstrukturen nur bei spezifischen und hochwertigen oder großen und sich häufig wiederholenden Transaktionen angemessen sind (Williamson 1990a, S. 69). Zudem ist zu berücksichtigen, dass es bei regelmäßig wiederkehrenden völlig oder nahezu identischen Transaktionen zu kostensenkenden Lerneffekten der Transaktionspartner kommen kann, was wiederum die Notwendigkeit von umfassenden Beherrschungsstrukturen reduziert (Picot 1982, S. 272). 


\subsubsection{Exkurs: Die fundamentale Transformation}

Der Ausgangspunkt zur Betrachtung der fundamentalen Transformation ist darin zu sehen, dass unterschiedliche Voraussetzungen für das Zustandekommen einer Tauschbeziehung und ihrer vertraglichen Absicherung existieren, welche von der Anzahl der potentiellen Anbieter beeinflusst werden.

$\mathrm{Zu}$ unterscheiden sind grundsätzlich die beiden Extrempunkte einer Monopolbeziehung, in der es nur einen Anbieter gibt und einer Konkurrenzbeziehung, welche durch eine Vielzahl von potentiellen Anbietern gekennzeichnet ist. Innerhalb der Transaktionskostenökonomik wird diese Situation als Bietprozess oder auch Bietkonkurrenz aufgefasst (Williamson 1990a, S. 70-72).

Im Zuge dessen wird die Analyse über den Zeitpunkt des Vertragsabschlusses hinaus erweitert und sie umfasst sowohl eine Ex-ante-Betrachtung als auch eine Ex-postBetrachtung. Der anfängliche Bietprozess setzt lediglich die Vertragsverhandlungen in Gang und zu einer umfassenden Beurteilung der Transaktionsbeziehung ist es notwendig, zusätzlich sowohl die Erfüllung des Vertrags als auch den neuerlichen Bietprozess bei einer möglichen Vertragsverlängerung einzubeziehen (Williamson 1990a, S. 70).

Hierbei wird in Frage gestellt, dass eine anfänglich hohe Zahl an Teilnehmern am Bietprozess auch in der Folge bestehen bleibt. Ob ein Wettbewerb auch ex-post wirksam ist, hängt davon ab, in welchem Umfang dauerhafte transaktionsspezifische Investitionen getätigt wurden. Für den Fall, dass eine derartige Spezifizierung nicht stattgefunden hat, ergeben sich für den Höchstbieter in weiteren Verhandlungen und Bietprozessen keinerlei Vorteile gegenüber den erfolglosen Konkurrenten und eine anfängliche Konkurrenzsituation bleibt auch zukünftig bestehen (Williamson 1990a, S. 70). Sind jedoch im Verlauf der Vertragsbeziehung Investitionen in transaktionsspezifische Faktoren erfolgt, ergibt sich daraus eine von der anfänglichen Konkurrenzsituation grundlegend verschiedene Situation. Der erfolgreiche Bieter hat eine von den Mitkonkurrenten unterschiedliche Ausgangssituation und eine ursprüngliche Konkurrenzsituation hat sich faktisch $\mathrm{zu}$ einer Situation eines bilateralen Angebots entwickelt.

Aus dieser von Williamson als fundamentale Transformation bezeichneten Umwandlung einer ex-ante unspezifischen in eine ex-post spezifische 
Transaktionsbeziehung ergeben sich weitreichende Konsequenzen für die vertragliche Ausgestaltung (Williamson 1990a, S. 70-71).

Durch die neu entstandene Entscheidungssituation ändern sich für die Kooperationspartner die Anreizstrukturen und die spezifischen Bindungen an den Transaktionspartner. Es entsteht eine Abhängigkeitsbeziehung, die nachträgliche Anreize für opportunistisches Verhalten eröffnet und eine bilaterale monopolistische Struktur aufweist. Durch die spezifische Transaktionsbeziehung wird der Wechsel zu anderen Handlungsalternativen durch die damit verbundenen Transaktionskosten erschwert oder gänzlich eingeschränkt. Neben einer bilateralen kann es ebenfalls zu einer einseitigen Monopolsituation kommen und der abhängige Vertragspartner wird in seinem eigenen Interesse versuchen, die Transaktionsbeziehung aufrecht zu erhalten, da alternative Vertragsbeziehungen zu einem Verlust finanzieller Werte führen würden (Williamson 1990a, S. 70-72).

Zusammenfassend ist eine fundamentale Transformation dann gegeben, wenn sich bedingt durch eine spezifische Transaktion ein Wandel weg von einer durch die freie Wahlmöglichkeit der Vertragspartner gekennzeichneten Konkurrenzsituation hin zu einer Abhängigkeit einstellt. ${ }^{62}$

\subsection{Beherrschung und Überwachung von Transaktionen}

Nachdem sowohl die grundlegenden Verhaltensannahmen als auch die Dimensionen von Transaktionen beschrieben wurden, wird mit der Betrachtung von Beherrschungsstrukturen das Ziel der institutionellen Ausgestaltung von Transaktionsbeziehungen eingeführt.

Bei der Suche nach einer effizienten Beherrschungsstruktur gilt es die damit verbundenen und auf die spezifischen Ausprägungen der Dimensionen von Transaktionen (Faktorspezifität, Unsicherheit, Häufigkeit) ${ }^{63}$ zurückzuführenden Transaktionskosten vor dem Hintergrund der zugrunde gelegten Verhaltensannahmen (begrenzte Rationalität, Opportunismus) zu bewerten.

\footnotetext{
${ }^{62}$ Die fundamentale Transformation und ihre praktische Relevanz wird in dieser Arbeit im Rahmen der Zuschlagserteilung in einem Ausschreibungsverfahren für Umweltleistungen in Kapitel Sechs erneut aufgegriffen.

${ }^{63}$ Wie oben erwähnt, wird die Unsicherheit realistischerweise als so hoch angesehen, dass sie nicht beeinflusst werden kann. Für die weiteren Ausführungen ist die Unsicherheit demzufolge als gegeben anzusehen und es finden ausschließlich die Faktorspezifität und die Häufigkeit Berücksichtigung.
} 
Dem vorangestellt wird eine knappe Übersicht über vertragsrechtliche Grundlagen, bei der Williamson folgend ebenfalls auf die dreiteilige Typologie des Vertrags von Macneil (1974; 1978) zurückgegriffen wird (Williamson 1975, S. 235-238; Williamson 1990a, S. 77-81).

\subsubsection{Vertragsrechtliche Grundlagen}

In Analogie zu der von Macneil vertretenen Einschätzung folgt die Transaktionskostenökonomik der Annahme, dass die Analyse einer isolierten Transaktions- bzw. Vertragsbeziehung im Sinne der eindeutigen Verknüpfung von Vereinbarung und Leistung realitätsfremd ist.

Vielmehr können Verträge mehrdeutig sein und erfordern eine differenzierte Betrachtung mit dem Augenmerk auf dem jeweiligen Vertragszweck (Macneil 1974, S. 738; Williamson 1975, S. 235-236; Williamson 1990a, S. 77; Furubotn und Richter 1991, S. 18-24). Hierzu werden klassische, neoklassische und relationale Verträge unterschieden und nachfolgend in ihren Grundzügen dargelegt.

\section{Klassischer Vertrag}

Der dem klassischen Vertragsrecht zuzurechnende vollständige Vertrag stellt das rechtswissenschaftliche Gegenstück des Modells eines vollkommenen Marktes dar. Die Identität der Vertragspartner findet keine Berücksichtigung und die gehandelten Leistungen und Gegenleistungen sind vollständig spezifiziert. Für derartige Transaktionen werden in der Regel kurzfristige Verträge abgeschlossen, bei denen formale Vertragsinhalte bedeutender sind als informelle Absprachen. Sollte es trotz der umfassenden und vorausschauenden Vertragsausgestaltung $\mathrm{zu}$ einer nicht vertragskonformen Erfüllung kommen, erfolgt eine strikte Durchsetzung der Vertragsinhalte und eine entsprechende Sanktionierung über Gerichte (Williamson 1975, S. 236-237; Williamson 1990a, S. 78).

\section{Neoklassischer Vertrag}

Innerhalb des neoklassischen Vertragsrechts kommt der Identität der Vertragspartner eine größere Bedeutung als in klassischen Verträgen zu. Die Vertragsinhalte sind nicht vollständig spezifiziert und auf ihre strikte Durchsetzung über Gerichte wird dann verzichtet, wenn ein Abbruch der Vertragsbeziehung für beide Vertragspartner mit weitreichenden Nachteilen verbunden wäre. Grundsätzlich besteht der 
Zusammenhang, dass mit einer steigenden Bedeutung der Identität der Vertragspartner und den zu erwartenden Schäden im Fall eines vorzeitigen Abbruchs der Leistungsbeziehung bei auftretenden Konflikten eine Einbeziehung einer neutralen dritten Partei als Schlichtungsinstanz vorgesehen ist (Williamson 1975, S. 237-238; Picot und Dietl 1993, S. 315; Williamson 1990a, S. 78-80).

\section{Relationaler Vertrag}

Unter relationalen Verträgen sind in der Regel auf Dauer angelegte Kooperationen zu verstehen, bei denen den Vertragspartnern eine große Bedeutung zukommt. Anstelle einer exakten Spezifizierung der Vertragsinhalte werden implizite oder auf gegenseitigem Vertrauen beruhende Vereinbarungen in den Vordergrund treten und da die Vertragsbeziehung dauerhaft angelegt ist, erfolgt eine Beilegung möglicher Unstimmigkeiten durch interne Konfliktlösungen (Williamson 1975, S. 238; Williamson 1996, S. 97-99).

\subsubsection{Beherrschungs- und Überwachungsstrukturen}

Nach der Einführung vertragsrechtlicher Grundlagen, werden die vier institutionellen Arrangements Marktkontrolle, dreiseitige Beherrschungsstruktur, zweiseitige Beherrschungsstruktur und (hierarchische) einseitige Beherrschungsstruktur betrachtet, im Rahmen deren Transaktionsbeziehungen in der Regel abgewickelt werden (Williamson 1990a, S. 81-89; Richter und Furubotn 2003, S. 193-198).

\section{Marktkontrolle}

Die Beherrschungsstruktur der Marktkontrolle in Form eines funktionierenden Marktes erweist sich dann als angemessen, wenn unspezifische und unpersönliche Transaktionen einmalig, gelegentlich oder regelmäßig abgewickelt werden.

Entscheidend ist hier die fehlende Faktorspezifität, denn somit besteht für die Abwicklung einer Transaktion keine notwendige Bindung an einen bestimmten Transaktionspartner und es existieren Marktalternativen durch eine Vielzahl potentiell gleichwertiger und substituierbarer Vertragspartner (Williamson 1990a, S. 83; Richter und Furubotn 2003, S. 196-197). Das zu einem Vertragsbruch führende Verhalten kann bei einer zukünftigen gleichartigen Transaktion dadurch sanktioniert werden, dass ein Vertrag mit einem neuen Transaktionspartner geschlossen wird und 
darüber hinaus die Reputation des vertragsbrüchigen Marktteilnehmers bekannt wird (Williamson 1990a, S. 83-84; Richter und Furubotn 2003, S. 593).

\section{Dreiseitige Beherrschungsstruktur}

Die dreiseitige Beherrschungsstruktur kommt dann zur Anwendung, wenn mittelund hochspezifische Transaktionen nur einmalig oder gelegentlich erfolgen.

Beeinflusst durch die Faktorspezifität und die bei der Suche eines neuen Transaktionspartners anfallenden hohen Transaktionskosten ist ein ausschließliches Verlassen auf die Sanktionskraft von Märkten nicht ausreichend. Daher ist neben den beiden Vertragsparteien die explizite Einbeziehung einer dritten Partei notwendig, die als neutrale Institution in Form einer Vermittlungs- oder Schiedsinstanz fungiert und sicherstellt, dass die Transaktion entsprechend der vertraglichen Vereinbarung abgewickelt wird (Williamson 1990a, S. 84-85; Richter und Furubotn 2003, S. 197).

\section{Zweiseitige Beherrschungsstruktur}

Dieser Ansatz betrachtet mittelspezifische Transaktionen, die sich regelmäßig wiederholen und zu deren Absicherung ein individueller zweiseitiger Vertrag geschlossen wird, der alle relevanten Aspekte abdeckt.

Dabei werden die Transaktionspartner nicht zusammengeschlossen und ihre rechtliche Selbständigkeit bleibt erhalten. Weiterhin können in Teilen sowohl marktliche Anreize wirksam werden und die regelmäßige Wiederholung der Transaktion, unter Beibehaltung der vertraglichen Ausgestaltung, trägt zu einer raschen Amortisation der anfänglichen Kosten der Vertragsausgestaltung bei (Williamson 1990a, S. 85-88; Richter und Furubotn 2003, S. 197).

\section{Einseitige Beherrschungsstruktur (Hierarchie)}

Das gegenteilige Extrem zur Marktkontrolle stellt die bei vollkommen spezifischen und sich regelmäßig wiederholenden Transaktionen zur Anwendung kommende einseitige Beherrschungsstruktur dar.

Hier erfolgt eine vollständige vertikale Integration hin zu einer hierarchischen Struktur, bei der die einzelnen Organisationseinheiten ihre rechtliche Unabhängigkeit aufgeben. Die notwendige Absicherung dieser hochspezifischen Transaktion kann dann bestmöglich unter Ausnutzung der sich bietenden Vorteile einer einheitlichen 
Struktur mit vereinheitlichten Interessen erfolgen (Williamson 1990a S. 88; Richter und Furubotn 2003, S. 197-198).

Abbildung 4-1 gibt einen Überblick des Zusammenwirkens der Verhaltensannahmen und der Dimensionen von Transaktionen, die je nach spezifischer Ausprägung eine der vier Beherrschungsstrukturen als angemessen und effizient erscheinen lassen.

Abb. 4-1: Beherrschungs- und Überwachungsstrukturen von Transaktionsbeziehungen

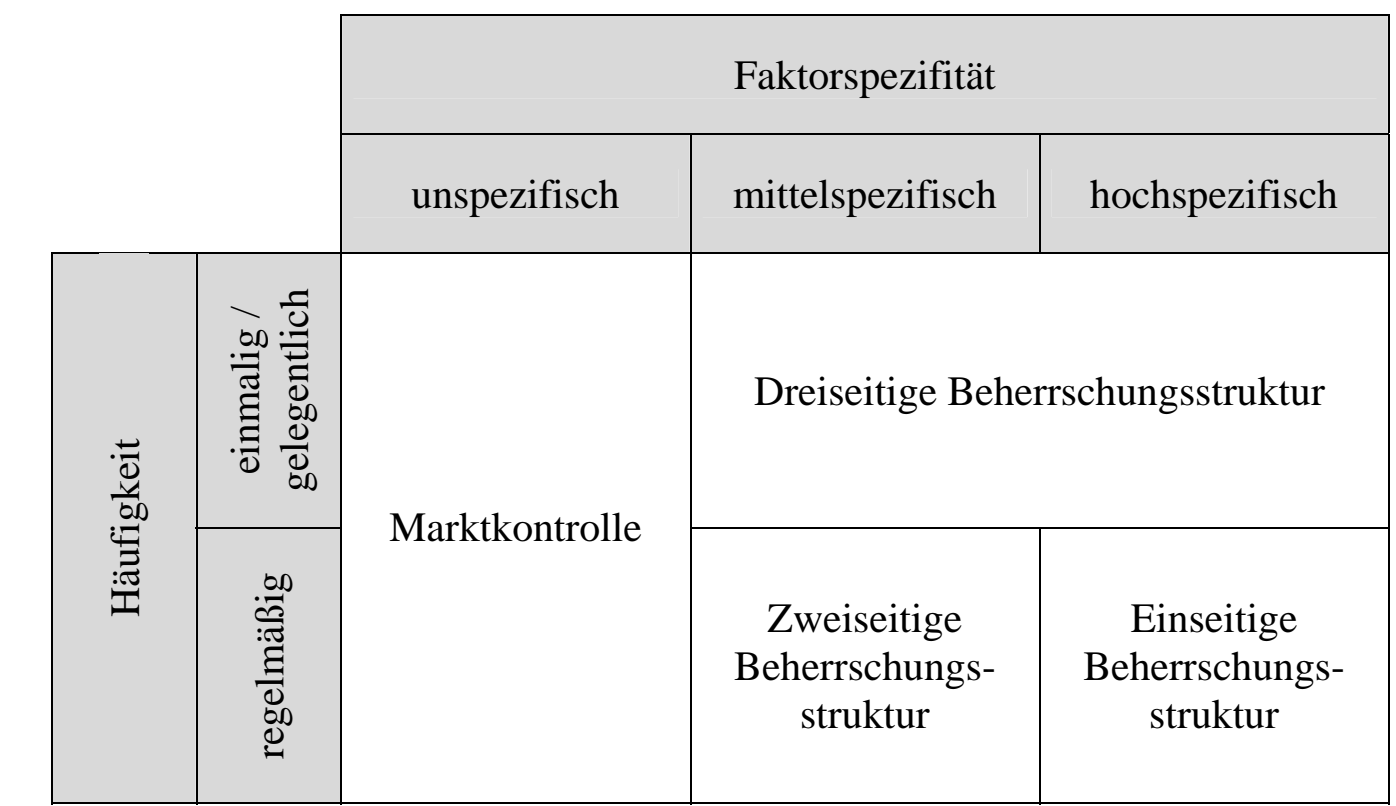

Quelle: Eigene Darstellung in Anlehnung an Williamson 1990a, S. 89.

Im Rahmen der vorliegenden Arbeit erfolgt eine kritische Würdigung der Beherrschungsstrukturen anhand ihrer Übertragbarkeit auf den Spezialfall eines Ausschreibungsverfahrens für ökologische Leistungen der Landwirtschaft und der damit verbundenen Transaktionsbeziehung in Kapitel Sechs.

\subsection{Abgrenzungen von Transaktionskosten}

Nachdem der theoretische Rahmen der Transaktionskostenökonomik dargelegt wurde, wird im Folgenden der Blick zunehmend auf praktische Fragen der Ermittlung und Bewertung von Transaktionskosten gerichtet.

Dazu wird eine Abgrenzung anhand der drei Kategorien Markttransaktionskosten, Unternehmenstransaktionskosten und politische Transaktionskosten vorgenommen (die Abgrenzung erfolgt in Anlehnung an Richter und Furubotn 2003, S. 57-65). 


\subsubsection{Markttransaktionskosten}

Die am häufigsten verwendete Abgrenzung von Transaktionskosten stellen die unter Markttransaktionskosten subsumierten Kosten dar, die in ihren Ursprüngen starke Bezüge zu Arbeiten von Coase (1960), Stigler (1961) und Dahlmann (1979) aufweisen.

Hier werden alle mit der Abwicklung von Transaktionen über Märkte relevanten Gesichtspunkte von der Suche eines Vertragspartners über den Abschluss und die Überwachung bis hin zu der Erfüllung eines Vertrags betrachtet (Coase 1960, S. 15). Im Gegensatz zur neoklassischen Sichtweise werden folglich keine Markthandlungen analysiert, welche als anonymer Tausch auf vollkommenen Wettbewerbsmärkten stattfinden, sondern es werden die in der Realität vorherrschenden Aspekte von Unsicherheit, unvollständiger Information und der Relevanz von Transaktionskosten einbezogen.

Mit dem Ziel, die Kosten der Marktnutzung auch im Hinblick auf die spätere Analyse im Rahmen dieser Arbeit weiter zu differenzieren, werden im Folgenden Such- und Informationskosten, Verhandlungs- und Entscheidungskosten, Überwachungs- und Durchsetzungskosten und Investitionen in Sozialkapital als vier Arten von Markttransaktionskosten unterschieden (Richter und Furubotn 2003, S. 59-61).

\section{Such- und Informationskosten}

Im Vorfeld der Abwicklung von Markttransaktionen und des Vertragsabschlusses ist es notwendig, potentielle Vertragspartner ausfindig $\mathrm{zu}$ machen. Hier sind exemplarisch Besuche von Messen und Informationsveranstaltungen oder das Studium einschlägiger Literatur zu nennen, was für die Anbahnung einer Transaktion zu unvermeidbaren Kosten führt und unentbehrlich ist, um mit zukünftigen Vertragspartnern in Kontakt zu treten.

Ist dieser Kontakt geknüpft, schließt sich die Notwendigkeit an, Informationen über die Leistungsfähigkeit der potentiellen Vertragspartner $\mathrm{zu}$ gewinnen und eine individuell bestmögliche Ausgangsposition für die folgenden Vertragsverhandlungen einnehmen zu können. Infolgedessen sind die Such- und Informationskosten auch als Kosten der Anbahnung von Verträgen zu bezeichnen. 


\section{Verhandlungs- und Entscheidungskosten}

Die in dieser Kategorie subsumierten Markttransaktionskosten können als Kosten des Abschlusses von Verträgen charakterisiert werden.

Dies umfasst insbesondere die Wahl einer adäquaten Beherrschungsstruktur und ihrer spezifischen Ausgestaltung, was je nach Komplexität sowohl mit einem erheblichen Zeitaufwand als auch mit der Notwendigkeit einer Einbeziehung von weiteren Akteuren verbunden sein kann. Die Entscheidungskosten beinhalten vor allem Kosten der Informationsaufbereitung, der Entlohnung von Beratern oder auch Kosten der Entscheidungsfindung innerhalb von Gruppen bis hin zur Wahl der Vertragsausgestaltung.

\section{III. Überwachungs- und Durchsetzungskosten}

Nach dem Vertragsabschluss ist es notwendig, diesen hinsichtlich der vereinbarten Qualität, den Lieferfristen oder der Bezahlung zu überwachen und bei einem abweichenden Verhalten gegebenenfalls rechtliche Schritte einzuleiten. Eine zentrale Rolle kommt auch hier den Informationskosten zu. Mit steigenden Überwachungsund Durchsetzungskosten nehmen demzufolge die Anreize für opportunistisches Verhalten und die Gefahr der Nichterfüllung von Verträgen zu.

\section{Investitionen in Sozialkapital}

Einen in diesem Kontext mitunter ungewöhnlichen Aspekt stellen die im Verlauf von Vertragsbeziehungen vorgenommenen Investitionen in Sozialkapital dar, welche die wirtschaftlich relevante soziale Struktur von Märkten betonen.

So sind Wirtschaftssubjekte sowohl privat als auch geschäftlich in soziale Beziehungen eingebunden, was aus Sicht der Transaktionskostenökonomik dazu beitragen kann, dass bei Markthandlungen komplexe Entscheidungen unter Unsicherheit und bei begrenzter Rationalität unter Zuhilfenahme bestehender sozialer Kontakte effizienter getroffen werden können. Dabei ist der Aufbau eines sozialen Netzwerks mit den hier angesprochenen Transaktionskosten verbunden. Langfristig kann dies wiederum dazu führen, dass je nach Art der Transaktionsbeziehung speziell die Such- und Informationskosten und die Verhandlungs- und Entscheidungskosten reduziert werden können, da weniger neue Kontakte aufgebaut werden müssen und sich die bestehenden Beziehungen durch ein über die Zeit gewachsenes Vertrauen auszeichnen. 
Im weiteren Verlauf der Arbeit wird bei der Ermittlung von Transaktionskosten der Landwirte im Zuge eines Ausschreibungsverfahrens für Umweltleistungen auf die Markttransaktionskosten Bezug genommen, wobei dort eine spezifische Abgrenzung erfolgt.

\subsubsection{Unternehmenstransaktionskosten}

Als zweite Kategorie von Transaktionskosten können die in Abgrenzung zu Markttransaktionskosten einer unternehmensinternen Sichtweise folgenden Unternehmenstransaktionskosten angesehen werden. ${ }^{64}$

In diesem Zusammenhang werden Unternehmen als alternative Koordinationsmechanismen zu Märkten angesehen und die Betrachtung beginnt mit dem Zeitraum nach Abschluss eines Vertrags, da alle Aktivitäten bis dahin zu den Markttransaktionskosten gezählt werden. Zu unterscheiden sind daher die folgenden Abgrenzungen (Richter und Furubotn 2003, S. 61-63).

\section{Kosten der Einrichtung, Erhaltung oder Änderung einer Unternehmung}

Diese in der Regel fixen Transaktionskosten ergeben sich bei allen die Organisationsstruktur einer Unternehmung betreffenden Aktivitäten und können unter anderem Investitionen in neue Unternehmensbereiche, Kosten im Zusammenhang mit der Durchführung oder Abwehr von Unternehmenszusammenschlüssen oder der Änderung der rechtlichen Unternehmensform umfassen.

\section{Kosten des Betriebs einer Unternehmung}

Die innerhalb einer bestehenden Organisationsstruktur anfallenden Transaktionskosten umfassen die mit der Überwachung und Evaluierung der Unternehmenstätigkeit verbundenen Informationskosten und die im Zusammenhang mit der physischen Übertragung von Gütern oder auch Dienstleistungen entstehenden Kosten. Als Beispiele für diese in der Prozesskostenrechnung ${ }^{65}$ erfassten

\footnotetext{
64 Im Verlauf dieser Arbeit und im Rahmen der Ermittlung von Transaktionskosten werden Unternehmenstransaktionskosten explizit nicht weiter betrachtet, weshalb hier lediglich eine knappe Übersicht gegeben wird.

${ }^{65}$ Das Ziel der Prozesskostenrechnung ist es, den Anteil der Transaktionskosten an den Gemeinkosten zu ermitteln, Einsparpotentiale aufzudecken und die Notwendigkeit von Transaktionen zu hinterfragen.
} 
Bestandteile sind Kosten der Zwischenlagerung von Halbfertigprodukten oder der Beschaffung und Aufbereitung produktionsrelevanter Daten anzuführen.

\subsubsection{Politische Transaktionskosten}

Die dritte Kategorie sind die als politische Transaktionskosten definierten Kosten der institutionellen Rahmenbedingungen.

Diese Rahmenbedingungen können verschiedenartig ausgestaltet sein und sind entscheidend für die in ihrem Geltungsbereich abzuwickelnden Unternehmens- und Markttransaktionen, so dass auch hier das Ziel keine unreflektierte Minimierung von politischen Transaktionskosten, sondern vielmehr die spezifische Analyse ihrer Notwendigkeit und praktischen Effizienz sein muss. So kann der Aufbau und die Existenz eines Rechtsrahmens oder einer Verwaltungsstruktur zu relativ hohen politischen Transaktionskosten führen, aber dadurch umfassende Rechtssicherheit bieten und sich positiv auf die Abwicklung von Unternehmens- und Markttransaktionen auswirken.

Je nach Untersuchungsgegenstand und betrachteter Institution können politische Transaktionskosten regional, national, europaweit oder auch weltweit auftreten und analog zu Unternehmenstransaktionskosten hinsichtlich der Implementierung institutioneller Rahmenbedingungen und ihres Betriebs unterschieden werden (Richter und Furubotn 2003, S. 63-65).

\section{Kosten der Implementierung, Erhaltung und Anpassung von institutionellen Rahmenbedingungen eines Gemeinwesens}

Hierzu sind alle Kosten zu zählen, die mit dem Ausbau der öffentlichen Verwaltung, der Agrarverwaltung, des Bildungswesens, des Militärs, der Rechtsordnung usw. in Verbindung stehen und ihre Handlungsfähigkeit herstellen.

\section{Kosten des Betriebs eines Gemeinwesens}

Nachdem die Institutionen des Gemeinwesens aufgebaut und handlungsfähig sind, erfüllen sie die ihnen übertragenen Aufgaben, wobei Transaktionskosten als laufende Kosten der Staatstätigkeit anfallen. $\mathrm{Zu}$ nennen sind beispielhaft Kosten der Durchführung von Agrarumweltprogrammen und Gesetzgebungsprozessen oder der Bereitstellung eines umfassenden Bildungsangebots. 
Es wird deutlich, dass die Kosten eines Gesetzes, eines Agrarumweltprogramms usw. nicht nur aus den unmittelbaren Staatsausgaben, sondern in einem mehr oder weniger großen Umfang auch aus politischen Transaktionskosten bestehen, die sich vor allem aus Such- und Informationskosten sowie Entscheidungs- und Überwachungskosten zusammensetzen.

Im Hinblick auf die Bereitstellung und Honorierung von Umweltleistungen kann in diesem Zusammenhang zugleich von den Kosten der Bereitstellung öffentlicher Güter gesprochen werden. Im Zuge dessen tritt der Staat als Vertreter der Bevölkerung auf und die politischen Transaktionskosten sind als Vertretungskosten anzusehen. ${ }^{66}$

\subsection{Kritik am Ansatz der Transaktionskostenökonomik}

Wie viele sich in der Ökonomik neu etablierende Ansätze wurde auch die Transaktionskostenökonomik im Verlauf ihrer Entwicklung vielfältig und mitunter massiv kritisiert. Einige wesentliche Kritikpunkte werden im Folgenden in vier thematische Gruppen unterteilt kurz dargestellt.

\section{Ungenaue begriffliche Abgrenzungen}

Ein elementarer Kritikpunkt besteht darin, dass zentrale Begriffe der Transaktionskostenökonomik, wie Transaktion oder Transaktionskosten, nur sehr ungenau definiert und verwendet werden können (Motyka 1989, S. 214-215; Currie und Messori 1998, S. 179; Gerhard 1995, S. 201-202; Allen 2000, S. 893-895).

Einer besonderen Kritik unterliegt der Begriff der Transaktionskosten, wie sie von Cook und Emerson hervorgebracht wird: „One can hardly imagine a more diffuse and non-discriminating treatment of what is offered as a major concept in an important theory. It is left to us to induce the meaning of the term from situationspecific examples they offer, but examples are never sufficient for theory-building purposes.“ (Cook und Emerson 1984, S. 23).

Dies macht deutlich, dass eine begrifflich ungenaue Verwendung von Transaktionskosten, sowohl in der Theoriebildung als auch in der praktischen Anwendung, zu Missverständnissen und Fehlinterpretationen führen kann, da zwar

\footnotetext{
${ }^{66}$ Eine Betrachtung der politischen Transaktionskosten des in der vorliegenden Arbeit verfolgten Ansatzes kann zum Zeitpunkt der hier relevanten Umsetzung noch nicht erfolgen und sie bleiben für die weiteren Untersuchungen unberücksichtigt.
} 
mit der Vergleichbarkeit von Transaktionskosten argumentiert wird, aber möglicherweise die als Transaktionskosten definierten Kosten zu vergleichender institutioneller Ausgestaltungen nicht vergleichbar sind.

Darüber hinaus ergeben sich im Rahmen einer dynamischen Betrachtung weitere Kritikpunkte. Zum einen hinsichtlich der Notwendigkeit, dass bei der Bewertung des Übergangs von einem institutionellen Arrangement zu einem anderen die zugrunde liegende Transaktion unverändert und vergleichbar bleibt (Dow 1987, S. 17-18; Yamin 1996, S. 166). Zum anderen ist die Problematik der erst zukünftig auftretenden Ex-post-Transaktionskosten zu nennen, welche unsicher sind und trotz einer unterstellten eindeutigen Kenntnis der zum Entscheidungszeitpunkt relevanten Transaktionskosten dazu führen, dass nur eine unvollständige Bewertung von alternativen Institutionen möglich ist (Leblebici 1985, S. 100-101).

\section{Mangelnde Qualität der empirischen Bestätigungen}

Speziell vor dem Hintergrund der sowohl durch die Transaktionskostenökonomik als auch die gesamte Neue Institutionenökonomik beanspruchten größeren Praxisnähe ihres Ansatzes stellt die bislang noch nicht umfassend ausgeprägte empirische Bestätigung und Anwendung einen weiteren Kritikpunkt beziehungsweise Forschungsbedarf dar (Granovetter 1985, S. 504; Wiggins 1991, S. 657-658; Börner und Macher 2001, S. 5-8; Richter 2001, S. 223-224; Wang 2003, S. 9).

So wird Williamson eine empirisch-induktive Argumentation in Form einer auf Fallbeispielen begründeten Bildung allgemeiner Muster vorgeworfen, welche als Beleg seiner Theorie herangezogen werden (Dow 1987, S. 34; Schneider 1995, S. 268). Darüber hinaus wird eine streckenweise Argumentation auf der Grundlage von Plausibilitätsüberlegungen kritisiert (Frese 1990, S. 16; Föhr 1991, S. 74-79). Diese erhöht die Angreifbarkeit des Theoriegebäudes und macht eine empirische Überprüfung des Analyserahmens notwendig, da prinzipiell jedes institutionelle Arrangement mit dem Verweis auf Transaktionskosten gerechtfertigt werden kann, wenn die herangezogenen Transaktionskosten weder genau bestimmt noch verglichen werden können (Börner und Macher 2001, S. 5-8). 


\section{Soziologische Kritik an den Verhaltensannahmen}

Ein weiterer Kritikpunkt bezieht sich auf die zugrunde gelegten Verhaltensannahmen und wird maßgeblichen von Soziologen vertreten. ${ }^{67}$

Eine wesentliche Kritik wird von Granovetter hervorgebracht und behandelt die aus seiner Sicht vernachlässigte Berücksichtigung der sozialen Eingebundenheit ökonomischer Aktivitäten. Granovetter kritisiert den von Williamson in „Markets and Hierachies“ beschriebenen Markt und bezeichnet ihn als einen realitätsfremden anonymen Markt aus neoklassischen Modellen, wie er in der Realität nicht anzufinden sei, da dort sämtliche Markttransaktionen in ein soziales Umfeld eingebunden sind (Granovetter 1985, S. 495).

Eine derartige Einbettung in soziale Strukturen und Netzwerke schaffe Vertrauen und schütze daher in einem höheren Maß vor opportunistischem Verhalten als es bei Williamson berücksichtigt wird (Granovetter 1985, S. 490-491). In der Folge führt dies in der Transaktionskostenökonomik zu einer Überbetonung der hierarchischen Beherrschungsstrukturen im Vergleich zu einem durch soziale Eingebettetheit geprägten Markt (Granovetter 1985, S. 495-496).

\section{IV. Ökonomische Kritik an der Wahl effizienter Institutionen}

Die von Williamson vertretene geplante Wahl institutioneller Arrangements mit dem Ziel der Reduzierung von Transaktionskosten und ihrer Überwachung vor dem Hintergrund spezifischer Verhaltensannahmen und Dimensionen von Transaktionen wird von Schneider (1985; 1995) dahingehend kritisiert, dass dies auch in der Realität ein effizienzorientiertes und rationales Verhalten der Individuen voraussetzt und darin ein Widerspruch innerhalb der Transaktionskostenökonomik zu sehen ist (Schneider 1995, S. 263-266).

Schneider argumentiert, dass wenn die begrenzte Rationalität einen wesentlichen Bestandteil der Transaktionskostenökonomik ausmacht, nicht nachvollziehbar sei, wie Individuen Institutionen in der Praxis rational und effizient wählen können (Schneider 1995, S. 265-266).

Dieser Argumentation ist nur bedingt zu folgen, denn wie in den vorherigen Ausführungen deutlich geworden ist, setzt eine rationale Wahl von Institutionen nach Williamson voraus, dass die Verhaltensannahmen Opportunismus und begrenzte

\footnotetext{
${ }^{67}$ Insbesondere von Perrow (1981; 1986); Granovetter (1985; 2002); Powell (1990) und Freeland (1996).
} 
Rationalität als Restriktionen anerkannt werden. Somit handeln Individuen auch bei der Wahl von institutionellen Ausgestaltungen lediglich intendiert oder begrenzt rational und es können ineffiziente Institutionen gewählt werden und unbeabsichtigte Folgen eintreten. $^{68}$

Die Kritik ist somit dahingehend zu widerlegen, dass Schneider eine ungenaue Interpretation des Transaktionskostenansatzes zugrunde legt und seinerseits in der Form inkonsistent argumentiert, dass der Analyserahmen der Transaktionskostenökonomik verlassen wird, um ihn dann mit einer nicht zutreffenden Auslegung zu kritisieren.

In den obigen Ausführungen wurde gezeigt, dass die Transaktionskostenökonomik bislang auf vielfältige Weise kritisiert wurde, wobei einige Kritikpunkte auf einer Reihe von Missverständnissen beruhen, die zu einem großen Teil mit unscharfen begrifflichen Abgrenzungen begründet werden können. Somit sind speziell die definitorischen und empirischen Aspekte weniger als endgültige Kritik, sondern vielmehr als Schwierigkeiten und bestehender Forschungsbedarf bei der praxisorientierten Anwendung der Transaktionskostenökonomik anzusehen.

${ }^{68}$ Die Frage der geplanten oder ungeplanten Institutionenwahl wird von Williamson beispielsweise in „The Mechanisms of Governance“ (1996) ausführlich diskutiert. 


\section{Ausschreibungen}

Auktionen und Ausschreibungen haben bislang in verschiedensten Bereichen und Ausgestaltungen einen praktischen Einsatz gefunden und ihre Funktion als Allokationsmechanismus wurde umfassend modelliert, dokumentiert und bewertet (McAfee und McMillian 1987; Wolfstetter 1996; Klemperer 2002). ${ }^{69}$

Der Einsatz von Ausschreibungen zur Honorierung von Umweltleistungen stellt jedoch ein noch relativ neues Anwendungsfeld dar, welches - zweifelsohne entscheidend beeinflusst durch die Implementierung des Conservation Reserve Program in den USA - in den letzten zwei Jahrzehnten ein steigendes Interesse erfahren hat (Latacz-Lohmann und Schilizzi 2005, S. 10-11). Dennoch fehlt es weiterhin an umfassenden praktischen Erprobungen und entsprechende Ausschreibungen wurden maßgeblich aus theoretischer Sicht sowie unter Zuhilfenahme von Simulationen und in Laborexperimenten diskutiert, wobei allenthalben ihr ökonomisches Potential für einen effizienteren Einsatz öffentlicher Finanzmittel gegenüber einer einheitlichen Prämienzahlung betont wird (LataczLohmann 1993, S. 357; Holm-Müller et al. 2002, S. 119; Cason und Gangadharan 2003, S. 18-20; Latacz-Lohmann und Hodge 2003, S. 136-137).

In den letzten Jahren haben diese wissenschaftlichen Argumente zunehmend auch in Europa Eingang in die agrarumweltpolitische Diskussion gefunden, was bislang in der Einbeziehung des Einsatzes von Ausschreibungen in Artikel 39 der ELERVerordnung gipfelt. Damit ist erstmals der Weg für eine Implementierung von Ausschreibungsverfahren in die nationale und europäische Agrarumweltpolitik ab 2007 bereitet.

Infolgedessen wird hier unter Berücksichtigung der theoretischen Vorarbeiten ausdrücklich eine praxisbezogene Herangehensweise gewählt und auf eine Darstellung der in der Literatur bereits umfangreich erfolgten formalen Analyse der Auktions- und Ausschreibungsoptionen verzichtet. Vielmehr ist es das Ziel, die grundlegenden Ausgestaltungsmöglichkeiten von Auktionen auf den Bereich der Ausschreibung von Umweltleistungen anzuwenden und hinsichtlich ihrer praktischen Umsetzbarkeit zu diskutieren.

\footnotetext{
69 Einen umfassenden Überblick geben unter anderem die Arbeiten von Klemperer (2002; 2004), Krishna (2002) und Jehiel und Moldovanu (2003).
} 
Das Kapitel einleitend wird in Abschnitt 5.1 auf die auktionstheoretischen Grundlagen und die zentralen Ausgestaltungsmöglichkeiten von Eingut- und Mehrgüterauktionen eingegangen.

Im Anschluss wendet sich der Blick dem Spezialfall des Ausschreibungsverfahrens für Umweltleistungen zu und es werden in Abschnitt 5.2 die Grundlagen und Ziele von Ausschreibungen dargestellt. Im weiteren Verlauf werden Eingut- und Mehrgüterausschreibungen abgeleitet und hinsichtlich ihrer praktischen Anwendbarkeit zur Honorierung ökologischer Leistungen diskutiert. Darüber hinaus erfolgt die Einbeziehung weiterer Ausgestaltungsmöglichkeiten und es wird das Ausschreibungsdesign der hier betrachteten Ausschreibungsverfahren eingeführt.

Das Kapitel abschließend wird ein Überblick über den bisherigen praktischen Einsatz von Ausschreibungen für Umweltleistungen gegeben, wobei exemplarisch drei Ansätze herausgegriffen werden. Dies sind das in den USA seit 1985 implementierte Conservation Reserve Program, welches in Abschnitt 5.3 ausführlich betrachtet wird. Darüber hinaus skizziert Abschnitt 5.4 Ausschreibungen innerhalb des BushTender Trial in Australien und zwei von der Universität Bonn in Nordrhein-Westfalen durchgeführte Modellausschreibungen.

\subsection{Auktionstheoretische Grundlagen}

Innerhalb der Wirtschaftswissenschaften handelt es sich bei der Auktionstheorie um einen noch relativ jungen Forschungszweig, was vor dem Hintergrund des in der Geschichte sehr lange bekannten und zunehmend weiter verbreiteten Allokationsmechanismus einer Auktion verwundert und gleichzeitig die Notwendigkeit seiner Betrachtung verdeutlicht (Wolfstetter 1998, S. 139-140; Krishna 2002, S. 1-2). ${ }^{70}$

Die erste theoretisch fundierte Abhandlung zu Auktionen ist die spieltheoretische Arbeit „Counterspeculation, Auctions, and Competitive Sealed Tenders“ (Vickrey 1961) aus dem Jahr 1961, welche Vickrey zu dem Begründer der Auktionstheorie gemacht hat (Moldovanu 1996, S. 656). ${ }^{71}$ Danach dauerte es bis in die 70er Jahre des vergangenen Jahrhunderts, bevor eine kontinuierliche ökonomische Analyse von

\footnotetext{
${ }^{70}$ Einen geschichtlichen Überblick über den frühen Einsatz von Auktionen gibt Shubik (1983).

${ }^{71}$ Für seine herausragenden Forschungsarbeiten auf diesem Gebiet wurde William Vickrey (zusammen mit James Mirrlees) im Jahr 1996 der Nobelpreis für Ökonomie verliehen.
} 
Auktionen begonnen und bis heute fortgeführt wurde (Klemperer 1999, S. 231; Stoneham 2003, S. 483). ${ }^{72}$

Der praktische Einsatz von Auktionen hat in der jüngeren Vergangenheit in zahlreichen Ausprägungen stark zugenommen, wobei exemplarisch die Versteigerung von Bundesschatzbriefen, Ölförderlizenzen, Tabak, Fisch, Blumen oder die öffentlichkeitswirksame Versteigerung von Funkfrequenzen und Mobilfunklizenzen (z.B. in Deutschland, Großbritannien, den Niederlanden und den USA) zu nennen sind (Krishna 2002, S. 1-2; Jehiel und Moldovanu 2003, S. 271272; Klemperer 2002, S. 196-172). Durch die aktuelle Verbreitung des Internets kann zudem mittlerweile nahezu jedes Gut unproblematisch und zu vergleichsweise geringen Transaktionskosten in einer Internet-Auktion gehandelt werden (Ariely et al. 2006, S. 1-3; Ockenfels und Roth 2006, S. 297-301).

Bevor im Detail auf Ausgestaltungsmöglichkeiten eingegangen wird, ist eine grundlegende begriffliche Abgrenzung von Auktionen und Ausschreibungen vorzunehmen.

Auktionstheoretisch sind beide Ausgestaltungsformen als Auktion zu charakterisieren und die Versteigerung eines Guts oder mehrerer Güter durch einen Auktionator stellt den Fall dar, der unter einer Auktion im engeren Sinn zu verstehen ist. Der in der Regel als Ausschreibung bezeichnete Fall ist der Einkauf eines Guts oder mehrerer Güter durch eine Vergabestelle (Moldovanu 1996, S. 652-653). Diese auch hier eingesetzte Variante ist auktionstheoretisch ebenfalls als umgekehrte Auktion oder Einkaufsauktion zu bezeichnen, wobei in den folgenden Ausführungen der in der Praxis gebräuchliche Begriff Ausschreibung beibehalten wird.

Darüber hinaus ist hinsichtlich der Anzahl der innerhalb einer Auktion oder eines Ausschreibungsverfahrens gehandelten Güter zu unterscheiden. Wird lediglich ein Gut angeboten (nachgefragt) ist von einer Eingutauktion (Eingutausschreibung) zu sprechen. Eine Mehrgüterauktion (Mehrgüterausschreibung) liegt vor, wenn mehrere Güter angeboten (nachgefragt) werden (Kräkel 1992, S. 106-108).

\footnotetext{
${ }^{72}$ Für einen Einblick in die Entwicklung der Auktionstheorie und die in diesem Zusammenhang zentralen Arbeiten siehe insbesondere die Übersichtsarbeiten von McAfee und McMillian (1987), Milgrom (1989), Wolfstetter (1996) und Klemperer (1999).
} 


\subsubsection{Gegenstand und Ziele von Auktionen}

Die Aufgabe und das Ziel eines Anbieters ist es, den für ihn optimalen Preis zu ermitteln und den Nachfrager auszuwählen, der das Gut oder die Güter zu diesem Preis kauft. Für den theoretischen Fall, dass der Verkäufer die Zahlungsbereitschaften der Nachfrager kennt, legt er einen nicht verhandelbaren Preis in Höhe der höchsten Zahlungsbereitschaft fest. Der Nachfrager mit dieser höchsten Zahlungsbereitschaft stellt den einzigen verbleibenden Interessenten dar und er erwirbt das Gut zu dem festgelegten Preis, so dass das Allokationsproblem trivial und effizient gelöst ist.

In der Realität ist die Entscheidungssituation des Anbieters jedoch durch eine Informationsasymmetrie zu seinen Lasten gekennzeichnet. $\mathrm{Da}$ ihm die Zahlungsbereitschaften der potentiellen Nachfrager nicht bekannt sind, besteht bei einer Festsetzung des Preises die Gefahr, dass der Preis zu hoch gesetzt wird, er die höchste Zahlungsbereitschaft übersteigt und das Gut nicht verkauft wird. Verlangt der Anbieter einen niedrigeren Preis, erhöht dies einerseits die Wahrscheinlichkeit, dass sich ein Nachfrager findet; andererseits sinkt im Zuge dessen der zu realisierende Erlös. Folglich gilt es für den Verkäufer diesen Trade-off zwischen Erlös und Verkaufswahrscheinlichkeit zu berücksichtigen und zu lösen.

Als praktische Alternative zur Setzung eines Preises kann der Anbieter eine Auktion durchführen und somit die bestehende Informationsasymmetrie verringern und sein Entscheidungsproblem bestmöglich lösen (Stoneham et al. 2003, S. 482-483). Eine Auktion ist dadurch gekennzeichnet, dass die als Bieter bezeichneten Nachfrager ein Gebot abgeben, zu dem sie bereit sind ein bestimmtes Gut zu kaufen. Im Verlauf einer Auktion bilden sich anschließend auf der Grundlage der eingegangenen Gebote der Verkaufspreis und der Vertragspartner heraus, wobei in der Regel der höchste Angebotspreis den Zuschlag erhält (Kräkel 1992, S. 8-10; Klemperer 1999, S. 230).

Eine weit verbreitete Definition von Auktionen geben McAfee und McMillian: „What is an auction? An auction is a market institution with an explicit set of rules determining resource allocation and prices on the basis of bids from market participants.“ (McAfee und McMillian 1987, S. 701).

Auktionen stellen demzufolge einen Allokations- und Preisfindungsmechanismus dar, der dadurch charakterisiert ist, dass die Abgabe der Gebote, die Zuschlagserteilung und somit auch die Preisbildung an ex-ante festgelegte Regeln 
gebunden ist und dies Auktionen von Verhandlungen abgrenzt (Wolfstetter 1998, S. 139). Wie beim Preissetzungsverfahren wird auch innerhalb einer Auktion der Anbieter aktiv und bietet hier den potentiellen Nachfragern ein Auktionsverfahren an, welches bis zur Zuschlagserteilung nicht mehr verändert werden kann und entweder in Form einer Gebotsabgabe akzeptiert oder durch eine Nichtteilnahme abgelehnt wird (Das und Sundaram 1997, S. 5).

Bevor das Auktionsdesign gewählt werden kann, gilt es die grundlegende Frage der Zielsetzung des Auktionsverfahrens zu beantworten, wobei insbesondere die folgenden drei Ziele abgeleitet werden können (Erhart 2005, S. 3):

I. Ertragsmaximierung: In der Auktionstheorie steht die Zielsetzung im Vordergrund, aus Sicht des Anbieters den Auktionserlös zu maximieren. In Abgrenzung zum Effizienzziel wird dann auch von einer optimalen Auktion gesprochen.

II. Effizienz: Das Effizienzziel einer Auktion ist als erfüllt anzusehen, wenn der Nachfrager mit der höchsten spezifischen Zahlungsbereitschaft den entsprechenden Zuschlag für ein Gut erhält.

III. Informationsgewinnung: Als drittes Ziel ist die mit einer Auktion verbundene Möglichkeit zu nennen, durch die individuellen Gebote ansonsten verborgene Informationen über die Präferenzen und Zahlungsbereitschaften der Bieter zu erlangen.

Zusammenfassend wird deutlich, dass der grundlegende Vorteil einer Auktion gegenüber einer Preissetzung darin besteht, dass die Preisbildung nach der Wahl des Auktionsdesigns auf der besser informierten Marktseite erfolgt und den Nachfragern ein Anreiz gegeben wird, Informationen über ihre Zahlungsbereitschaften zu offenbaren (Kräkel 1992, S. 8-9).

\subsubsection{Eingutauktionen}

Der zunehmende Einsatz von Auktionen und im Zuge dessen ihre fortschreitende wissenschaftliche Analyse hat dazu geführt, dass mittlerweile eine Vielzahl differenzierter und komplexer Ausgestaltungen von Auktionen existiert und diskutiert wird. Im Vordergrund der Betrachtung stehen jedoch vier so genannte Standardauktionen als Eingutauktionen, welche jeweils die Basis einer verfeinerten 
Ausgestaltung darstellen (Kräkel 1992, S. 13; Klemperer 1999, S. 229-331). Diese Standardauktionen lassen sich hinsichtlich der Gebotsabgabe anhand von zwei grundlegenden Merkmalen charakterisieren (Klemperer 1999, S. 229; Krishna 2002, S. 2-3).

Ein Kennzeichen ist die Frage, wie die Gebote abgegeben werden und ob ihr Inhalt den konkurrierenden Bietern vor der Zuschlagserteilung bekannt wird. Somit ist zwischen offenen Geboten (open auction) und verdeckten Geboten (sealed bid auction) zu unterscheiden. Bei der Abgabe von offenen Geboten werden die Gebote und die Bieter unmittelbar bekannt gegeben und die entsprechenden Informationen stehen allen Bietern und dem Auktionator zur Verfügung. Bei verdeckten Geboten erfolgt eine Gebotsabgabe ohne Kenntnis der konkurrierenden Bieter und ihrer Gebote.

Eine zweite Unterscheidung ergibt sich daraus, ob je Bieter ein Gebot oder sukzessive mehrere Gebote abgegeben werden können. Bei einer einmaligen Gebotsabgabe besteht lediglich die Möglichkeit, dieses unmittelbar entscheidungsrelevante Gebot bis zu einem bestimmten Zeitpunkt abzugeben. Bei einer mehrfachen Gebotsabgabe wird zudem die Option eröffnet, bis zur endgültigen Zuschlagserteilung auf die vorhergehenden Gebote anderer Bieter und den jeweils aktuellen Preis zu reagieren.

Aus diesen Überlegungen ergeben sich mit der Englischen Auktion, der Holländischen Auktion, der Höchstpreis-Auktion und der Zweitpreis-Auktion die innerhalb der Auktionstheorie unterschiedenen und nachfolgend betrachteten Standardauktionen (Vickrey 1961, S. 14-23; McAfee und McMillian 1987, S. 702; Kräkel 1992, S. 13-14; Klemperer 1999, S. 229; Krishna 2002, S. 11-162).

\section{Englische Auktion}

Bei der Englischen Auktion werden die Gebote offen abgegeben und ausgehend von einem niedrigen Ausgangsgebot wird das Gebot durch den Auktionator so lange gesteigert, bis keiner der Bieter mehr bereit ist eine weitere Erhöhung zu akzeptieren. Jeder Bieter kann im Verlauf der Auktion mehrere Gebote abgeben, wobei das jeweils aktuelle Höchstgebot überboten werden muss. Der Bieter, welcher das höchste Gebot abgegeben hat, erhält den Zuschlag und zahlt den entsprechenden Preis. Ein wesentlicher Unterschied der Englischen Auktion im Vergleich zu den 
anderen drei Standardauktionen ist dadurch gegeben, dass die Bieter hier aus den Geboten der anderen Bieter lernen und das eigene Gebot unter Rückgriff auf die neu gewonnenen Informationen sukzessive korrigieren können. ${ }^{73}$

\section{Holländische Auktion}

Im Gegensatz zur Englischen Auktion eröffnet der Auktionator das Bietverfahren bei der Holländischen Auktion mit einem sehr hohen Verkaufspreis. Daraufhin werden die Gebote so lange in kleinen Schritten reduziert, bis ein Bieter bereit ist das Gebot zu akzeptieren und ihm wird der Zuschlag erteilt. Die aktuelle Höhe des Gebots wird durch den Auktionator ausgerufen oder anderweitig öffentlich gemacht und wie bei der Englischen Auktion erfolgt die Gebotsabgabe offen. Für den Fall, dass sich bis zum Erreichen des Mindestgebots kein Bieter gefunden hat, wird das Bietverfahren mangels Geboten erfolglos beendet. Mit der Absenkung des Verkaufspreises steigt die Wahrscheinlichkeit, dass ein Bieter bereit ist, ein Gebot abzugeben und anders als bei der Englischen Auktion haben die Bieter keine Möglichkeit auf das Gebot zu reagieren, denn mit dem ersten Gebot wird unmittelbar der Zuschlag erteilt. Somit beinhaltet die Holländische Auktion ein in der Form von den anderen drei Standardauktionen grundlegend unterschiedliches Vorgehen, weil nicht »von unten nach oben« sondern »von oben nach unten « geboten wird. ${ }^{74}$

\section{Höchstpreis-Auktion}

Bei der Höchstpreis-Auktion gibt jeder Bieter ein einmaliges verdecktes Gebot ab und der Zuschlag wird dem höchsten Gebot erteilt. Nach der Zuschlagserteilung zahlt der Bieter den seinem Höchstgebot entsprechenden Preis und im Gegensatz zu der Englischen Auktion können die Bieter keinerlei Kenntnis über die Inhalte der anderen Gebote erlangen. Darüber hinaus besteht ebenfalls keine Möglichkeit, auf die Gebote anderer Bieter zu reagieren und das einmalige Gebot ist unmittelbar entscheidungsrelevant. ${ }^{75}$

\footnotetext{
73 In der englischsprachigen Literatur wird die Englische Auktion wegen ihrer ansteigenden Gebotspreise auch als „,ascending bid auction“ bezeichnet. Die Englische Auktion stellt die gebräuchlichste Auktionsform dar und als klassisches Beispiel sind hier Kunstauktionen zu nennen.

${ }^{74}$ In Abgrenzung zur Englischen Auktion wird die Holländische Auktion in der englischsprachigen Literatur als „descending bid auction“ bezeichnet und als praktisches Beispiel einer Holländischen Auktion liegt es auf der Hand, den Verkauf von frischen Schnittblumen in den Niederlanden zu nennen.

${ }^{75}$ Praktische Beispiele für die auch als „first-price sealed bid auction“ bezeichnete HöchstpreisAuktion sind Internet-Auktionen mit einer einmaligen Gebotsabgabe.
} 


\section{Zweitpreis-Auktion}

Ebenso wie bei der Höchstpreis-Auktion geben die Bieter bei einer ZweitpreisAuktion ein einmaliges verdecktes Gebot ab und der Bieter mit dem höchsten Gebot erhält den Zuschlag. Im Gegensatz zur Höchstpreis-Auktion muss der erfolgreiche Bieter jedoch nicht seinen Gebotspreis zahlen, sondern lediglich den Betrag, der dem zweithöchsten eingegangenen Gebot entspricht. ${ }^{76}$

\subsubsection{Mehrgüterauktionen}

Neben den Eingutauktionen besteht für einen Auktionator die Möglichkeit im Rahmen einer Auktion mehrere identische oder nicht identische Güter zum Verkauf anzubieten. Diese Mehrgüterauktionen haben in der Auktionstheorie, verglichen mit den Eingutauktionen, bislang relativ wenig Beachtung gefunden und stellen den aktuellen und sich in stetiger Weiterentwicklung befindlichen Forschungsschwerpunkt dar (Klemperer 1999, S. 240-241; Krishna 2002, S. 165166), weshalb sich in der Literatur noch keine einheitliche Bezeichnung der Mehrgüterauktionen etabliert hat.

Hier wird nachfolgend weitgehend auf die von Klemperer (1999), Krishna (2002) und auch auf die von Erhart (2005) gewählte Systematik zurückgegriffen und hinsichtlich der deutschen Bezeichnung Erhart (2005) gefolgt. Die sich daraus ergebenden Verfahren zu Mehrgüterauktionen werden im Folgenden erläutert (Klemperer 1999, S. 240-243; Krishna 2002, S. 165-250; Erhart 2005, S. 14-18).

\section{Sequentielle Auktion}

Die erste betrachtete Ausgestaltungsmöglichkeit ist eine sequentielle Auktion, bei der mehrere Güter nacheinander versteigert werden. Die sequentielle Auktion stellt die in der Praxis gängigste Form einer Mehrgüterauktion dar und kann dahingehend unterschieden werden, ob zeitgleich jeweils ein oder mehrere Güter versteigert werden. Für die Ausgestaltung einer Auktionsrunde kann für den Fall, dass jeweils nur ein Gut versteigert wird von einer wiederholten Eingutauktion gesprochen werden und eine der im vorherigen Abschnitt beschriebenen Standardauktionen Anwendung finden. Für den Fall, dass zeitgleich mehrere Güter zur Versteigerung

\footnotetext{
76 Die Zweitpreis-Auktion trägt zu Ehren ihres Entwicklers William Vickrey auch den Namen Vickrey-Auktion und wird in der englischsprachigen Literatur als „second-price sealed bid auction“ bezeichnet. Der praktische Einsatz der Zweitpreis-Auktion ist bislang noch wenig ausgeprägt.
} 
angeboten werden, liegt eine wiederholte Mehrgüterauktion vor, zu deren Durchführung jeweils eine der nachfolgenden Mehrgüterauktionen gewählt werden kann. $^{77}$

\section{Mengentender}

Diese auch als „Fixed-Price-Tender“ bezeichnete Mehrgüterauktion zeichnet sich dadurch aus, dass mehrere identische Güter zum Kauf angeboten werden. Seitens des Auktionators wird am Anfang der Auktion ein Preis je Einheit genannt und die Bieter können anschließend bis zu einem vorab festgelegten Termin unabhängig voneinander ein Gebot abgeben, welche Menge sie zu dem fixen Preis nachfragen. Für den Fall, dass die Nachfrage die angebotene Gesamtmenge nicht übersteigt, erhält jeder Bieter einen Zuschlag in Höhe der von ihm nachgefragten Menge. Übertrifft die Nachfrage das zur Verfügung stehende Angebot, erhalten die Bieter anteilig zu ihren Geboten einen Teil der Gesamtmenge. In beiden Fällen haben die Bieter den vorab gesetzten Preis je Einheit zu zahlen. ${ }^{78}$

\section{Simultane Auktionen mit Preis-Mengen-Geboten}

Im Gegensatz zu einem Mengentender, bei dem der Preis vorab gesetzt ist und das Gebot lediglich aus der nachgefragten Menge besteht, werden nun einmalig durchgeführte Mehrgüterauktionen betrachtet, deren individuelle Gebote sowohl eine Mengen- als auch eine Preiskomponente umfassen.

Bei einer simultanen Auktion mit Preis-Mengen-Geboten werden mehrere identische Güter zum Verkauf angeboten und die Bieter können innerhalb eines festgelegten Zeitraums unabhängig voneinander ein oder mehrere verschlossene Angebote abgeben, zu welchem Preis pro Einheit sie welche Menge nachfragen. Die Zuschlagserteilung erfolgt beginnend mit dem höchsten Preis »von oben nach unten« bis entweder die gesamte Angebotsmenge erschöpft ist oder alle eingegangenen Gebote einen Zuschlag erhalten haben.

Doch welchen Preis müssen die erfolgreichen Bieter letztlich zahlen? Diesbezüglich sind die drei Ausgestaltungsmöglichkeiten der Einheitspreis-Auktion, der preisdiskriminierenden Auktion und der Vickrey-Mehrgüterauktion zu betrachten.

\footnotetext{
77 Eine praktische Anwendung haben sequentielle Auktionen bislang beispielsweise bei Zwangsversteigerungen oder auch bei Kunstauktionen gefunden.

${ }^{78}$ Als Praxisbeispiel eines Mengentenders sind Aktienemissionen zu nennen.
} 


\section{IIIa. Einheitspreis-Auktion}

Bei der in der englischsprachigen Literatur als „Uniform-Price-Auction“ bezeichneten Einheitspreis-Auktion zahlen alle erfolgreichen Bieter nach Abschluss der Auktion unabhängig von ihrem Gebot einen einheitlichen Preis für jede erworbene Einheit. Entscheidungsrelevant für die Zuschlagserteilung ist weiterhin der individuell gebotene Preis und alle Bieter, deren Gebote einen bestimmten Preis überschritten haben, erhalten einen Zuschlag. Der zu zahlende Einheitspreis wird seitens des Auktionators festgesetzt, wobei in der Regel die Möglichkeiten bestehen, dass der Einheitspreis dem niedrigsten noch berücksichtigten oder dem höchsten nicht mehr berücksichtigten Gebot entspricht. ${ }^{79}$

\section{IIIb. Preisdiskriminierende Auktion}

Das konzeptionelle Gegenteil zu einer Einheitspreis-Auktion ist die preisdiskriminierende Auktion oder auch „Discriminative-Price-Auction“. Bei einer preisdiskriminierenden Mehrgüterauktion können die Bieter jeweils ein individuelles Gebot oder mehrere individuelle Gebote für identische Güter abgeben, welche die nachgefragte Menge und einen entsprechenden Preis pro Einheit umfassen. Dabei besteht zudem die Möglichkeit unterschiedliche Preise zu bieten, so dass jeder Bieter mehrere Zuschläge erhalten kann. Für jedes erfolgreiche Gebot zahlt der Bieter im Anschluss den jeweils von ihm gebotenen Preis und es ist folglich möglich und zu erwarten, dass es sowohl für die einzelnen Bieter als auch für verschiedene Gebote eines Bieters zu unterschiedlichen Preisen kommt. ${ }^{80}$

\section{IIIc. Zweitpreis-Mehrgüterauktion}

Die ebenso als „Vickrey-Mehrgüterauktion“ bekannte Zweitpreis-Mehrgüterauktion stellt das konzeptionelle Pendant zur Zweitpreis-Auktion dar. Erneut können die Bieter verschiedene Gebote für mehrere identische Güter abgeben, welche sowohl eine Preis- als auch eine Mengenkomponente beinhalten. Die eingegangenen Gebote werden den Angebotspreisen entsprechend in eine Rangfolge gebracht und die über

\footnotetext{
${ }^{79}$ Aktuell hat der Grundgedanke einer Einheitspreis-Auktion auch innerhalb von Online-Auktionen Anwendung gefunden. So bietet das Online-Auktionshaus eBay die Möglichkeit, mindestens zwei identische Güter in einer so genannten Multiauktion anzubieten. Bei einer Multiauktion kann es mehrere erfolgreiche Bieter geben, deren Höchstgebote für die Zuschlagserteilung entscheidend sind. Alle Bieter mit einem gebotenen Preis gleich oder über dem Höchstgebot erhalten einen Zuschlag und zahlen nach Abschluss der Auktion einen einheitlichen Preis für jedes erworbene Gut.

${ }^{80}$ Einen praktischen Einsatz findet die preisdiskriminierende Auktion bei Refinanzierungsgeschäften.
} 
einer Ausschlussgrenze liegenden Gebote erhalten einen Zuschlag. Zu zahlen hat ein erfolgreicher Bieter nun nicht die seinem Gebot entsprechenden Preise, sondern die Preise der Gebote für die Einheiten des Gutes, die gerade noch angenommen worden wären, wenn der erfolgreiche Bieter nicht an der Auktion teilgenommen hätte. Dementsprechend zahlt der Bieter die Preise der durch seine Gebote unterliegenden Gebote und entrichtet im Ergebnis die gesellschaftlichen Opportunitätskosten für seine Teilnahme. ${ }^{81}$

\section{Simultane mehrperiodige Auktion}

Als letzte Ausgestaltungsmöglichkeit wird mit der simultanen mehrperiodigen Auktion oder auch „Simultaneous-Multiple-Round-Auction“ eine konzeptionelle Verknüpfung der Englischen Auktion mit der Höchstpreis-Auktion betrachtet. Innerhalb dieser Auktionsform werden nacheinander zeitlich begrenzte Auktionsrunden durchgeführt, in denen die Bieter Gebote für mehrere Güter abgeben können. Nach Abschluss einer Auktionsrunde werden die Gebote differenziert nach den unterschiedlichen Gütern in eine Rangfolge gebracht und die jeweiligen Höchstgebote ermittelt. Diese Höchstgebote werden allen teilnehmenden Bietern bekannt gegeben und können in der kommenden Auktionsrunde überboten werden. Die Auktion ist beendet, wenn sich kein Bieter mehr findet, der die jeweils aktuellen Höchstgebote überbietet. ${ }^{82}$

\subsubsection{Auktionsmodelle und Äquivalenzeigenschaften}

Neben den obigen Aspekten beschäftigt sich die Auktionstheorie zudem mit der Frage, unter welchem Informationsstand die Bieter ihre Gebote abgeben und den sich daraus für die Ausgestaltung von Auktionen ergebenden Anforderungen. Hierzu werden nachfolgend mit dem Auktionsmodell bei Präferenzunsicherheit und dem Auktionsmodell bei Qualitätsunsicherheit die grundlegenden Auktionsmodelle ${ }^{83}$ kurz

\footnotetext{
81 Aufgrund ihrer hohen Komplexität ist die Zweitpreis-Mehrgüterauktion bislang eher als theoretisches Konstrukt anzusehen.

${ }^{82}$ Mehrperiodige Auktionen wurden in der Praxis bislang unter anderem für die Vergabe von Telekommunikationslizenzen - beispielsweise der UMTS-Lizenzen im Jahr 2000 - eingesetzt.

83 Über die beiden grundlegenden Auktionsmodelle hinaus existieren zudem Mischformen wie das auch als „Affiliated-Values-Model“ bezeichnete Milgrom-Weber-Modell, welches sowohl das Auktionsmodell bei Präferenzunsicherheit als auch das Auktionsmodell bei Qualitätsunsicherheit als Spezialfall beinhaltet und verschiedene Kombinationen von Präferenz- und Qualitätsunsicherheit betrachtet (Kräkel 1992, S. 95-97).
} 
betrachtet (Kräkel 1992, S. 10-12; Römhild 1997, S. 29; Wolfstetter 1998, S. 141143; Klemperer 1999, S. 229-230; Krishna 2002, S. 3-4). ${ }^{84}$

\section{Ia. Auktionsmodell bei Präferenzunsicherheit}

Das in der englischsprachigen Literatur als „Independent-Private-Values-Model“ bezeichnete Auktionsmodell bei Präferenzunsicherheit beruht auf der Annahme, dass für das zu versteigernde Gut kein einheitlicher und objektiv zu bestimmender Wert existiert und demzufolge jeder Bieter über eine individuelle Wertschätzung verfügt. Diese Wertschätzung entspricht der maximalen Zahlungsbereitschaft und wird als Reservationswert bezeichnet. Darüber hinaus wird unterstellt, dass der individuelle Reservationswert jeweils nur dem Bieter selber bekannt ist und eine Gebotsabgabe in Unkenntnis der Wertschätzungen aller weiteren Bieter erfolgt. Die zentrale Fragestellung innerhalb des Auktionsmodells ist daher die Wahl des optimalen Auktionsdesigns, welches aus Sicht des Auktionators dazu führt, dass der erwartete Auktionserlös maximiert wird. ${ }^{85}$

\section{Ib. Auktionsmodell bei Qualitätsunsicherheit}

Das zweite betrachtete Modell ist das Auktionsmodell bei Qualitätsunsicherheit, welches auch als „Common-Values-Model“ charakterisiert wird. Die grundlegende Annahme besagt hierbei, dass für den Auktionsgegenstand ein einheitlicher Wert existiert, dieser Reservationswert den Bietern jedoch zum Zeitpunkt der Gebotsabgabe noch unbekannt ist und sich die Gebote an einer individuellen Schätzung über die Höhe des Werts orientieren. Im Gegensatz zum Auktionsmodell bei Präferenzunsicherheit kann sich diese individuelle Wertschätzung, und somit das Gebot, auch an den Wertschätzungen anderer Bieter und weiteren Informationen orientieren, so dass als zentrale Fragestellung hier das Bieterverhalten bei einem gegebenen Auktionsdesign untersucht wird. ${ }^{86}$

\footnotetext{
${ }^{84}$ Vor dem Hintergrund der hier verfolgten praxisorientierten Ausrichtung der Arbeit wird auf eine vertiefte und formale Betrachtung dieser maßgeblich modelltheoretisch relevanten Aspekte verzichtet. 85 Als Beispiele für Auktionen mit Präferenzunsicherheit sind Versteigerungen von Kunstgegenständen als Sammlerobjekte zu nennen, die nicht zum Weiterverkauf erworben werden. Im Kontext der vorliegenden Arbeit sind zudem in der Regel auch Ausschreibungen von öffentlichen Aufträgen anzuführen, bei denen analog zu den Zahlungsbereitschaften die Kosten der Leistungserbringung ausschließlich dem Anbieter bekannt sind und die Grundlage des jeweiligen Angebots darstellen.

${ }^{86}$ Eine entsprechende Situation ist in der Praxis beispielsweise bei der Vergabe von Nutzungsrechten an Ölquellen gegeben, wenn die Bieter bei der Auktion lediglich über eine Schätzung verfügen und der tatsächliche Wert der Rohstoffe erst zu einem späteren Zeitpunkt bestimmt werden kann.
} 


\section{II. Äquivalenzeigenschaften von Auktionen}

Zusammenfassend ergibt sich aus den obigen Überlegungen im Hinblick auf die Äquivalenzeigenschaften der in Abschnitt 5.1.2 betrachteten Standardauktionen, dass Holländische Auktionen (offene Gebote) und Höchstpreis-Auktionen (verdeckte Gebote) über eine strategische Äquivalenz in dem Sinn verfügen, dass sich die Bieter jeweils identisch verhalten (Moldovanu 1996, S. 653-654). Dies ist darin begründet, dass den Bietern in beiden Auktionen bis zur Zuschlagserteilung keine Informationen über die anderen Bieter bekannt werden, sie sich ausschließlich an ihrer eigenen Wertschätzung orientieren können und der Gewinner der Auktion letztlich einen Preis in Höhe seines eigenen Gebots zahlen muss. Folglich ist diese Äquivalenz unabhängig davon, ob es sich um eine Auktion mit Präferenz- oder Qualitätsunsicherheit handelt (Krishna 2002, S. 4-5).

Demgegenüber liegt zwischen der Englischen Auktion (offene Gebote) und der Zweitpreis-Auktion (verdeckte Gebote) eine schwach ausgeprägte Äquivalenz vor, da beide nur für den Fall zu identischen Ergebnissen kommen, in dem alle Bieter ausschließlich über eine private Wertschätzung ohne weitergehende Informationen verfügen. Im Ergebnis wird dann derjenige Bieter mit der höchsten Zahlungsbereitschaft den Zuschlag zu dem der zweithöchsten Zahlungsbereitschaft entsprechenden Preis erhalten; bei der Englischen Auktion zuzüglich einer Bietmarge. Zu einem unterschiedlichen Ergebnis kommt es hingegen für den Fall, dass weitere Informationen einbezogen werden können und bei der offenen Englischen Auktion während des Bietvorgangs das eigene Gebot als Reaktion auf andere Gebote erhöht werden kann, während dies bei der Zweitpreis-Auktion nicht möglich ist (Krishna 2002, S. 5). ${ }^{87}$

\footnotetext{
${ }^{87}$ Für weitergehende Betrachtungen und Fragen der Äquivalenz von Auktionen in unterschiedlichen Auktionsmodellen sei an dieser Stelle exemplarisch auf die Arbeiten von McAfee und McMillian (1987), Bulow und Roberts (1989), Kräkel (1992) und Krishna (2002) verwiesen.
} 


\subsection{Ausschreibungen für Umweltleistungen}

Nachdem im vorherigen Abschnitt die auktionstheoretischen Grundlagen behandelt wurden, wendet sich der Blick nun spezifisch den Ausschreibungen für Umweltleistungen zu.

Wie bereits angesprochen wurde, stellt dieser Einsatz von Ausschreibungen einen theoretisch viel diskutierten, aber praktisch bislang noch wenig umgesetzten und untersuchten Sonderfall dar (Cason und Gangadharan 2003, S. 1-5). Dies hat zur Folge, dass sich Aussagen hinsichtlich der zu erwartenden ökonomischen Vorteile von Ausschreibungen gegenüber einer einheitlichen Prämienzahlung vor allem auf theoretisch evidente und in Simulationen nachgewiesene Effizienzvorteile beziehen (Latacz-Lohmann und Van der Hamsvoort 1997, S. 416-417; Cason und Gangadharan 2003, S. 18-20). ${ }^{88}$

Bevor im weiteren Verlauf der Arbeit der praktische Einsatz von Ausschreibungen dominiert, erfolgt in diesem Abschnitt die Verknüpfung theoretischer Aspekte mit dem Anwendungsgebiet der Ausschreibung ökologischer Leistungen der Landwirtschaft. Dazu werden die im vorherigen Abschnitt betrachteten Eingut- und Mehrgüterauktionen aufgegriffen und anhand ihrer grundlegenden Charakteristika auf den Bereich der Ausschreibung von Umweltleistungen übertragen. Darüber hinaus werden ergänzend weitere ausschreibungsspezifische Aspekte erörtert, wobei sich die Diskussion auf die im Kontext dieser Arbeit relevanten Ausgestaltungsmöglichkeiten beschränkt.

${ }^{88}$ Ein im Bereich des Einsatzes von Ausschreibungen zur Honorierung von Umweltleistungen der Landwirtschaft viel beachteter theoretischer Ansatz ist ein mikroökonomisches Modell, das auf den Arbeiten von Latacz-Lohmann (1993) und Latacz-Lohmann und Van der Hamsvoort (1997; 1998) beruht. Unter den dabei gewählten spezifischen Modellannahmen wurde in einer Simulation gezeigt, dass durch den Einsatz eines Ausschreibungsverfahrens im Vergleich zu einer Einheitsprämie $9 \%$ mehr Programmteilnehmer erreicht, die Emissionen um 4 \% verringert und die Programmausgaben um $2 \%$ reduziert werden konnten. Dabei wurden von Latacz-Lohmann und Van der Hamsvoort folgende Modellannahmen zugrunde gelegt (Latacz-Lohmann und Van der Hamsvoort 1997; 1998): a) keine speziellen höheren Transaktionskosten durch den Einsatz einer Ausschreibung; b) keine Ausprägung von Common Knowledge (die Preiskalkulation erfolgt ausschließlich anhand der individuellen Kostenstrukturen der Bieter); c) Symmetrie unter den Bietern; d) Konkurrenz zwischen den Bietern. 


\subsubsection{Grundlagen und Ziele}

Zur Abgrenzung von Auktionen und Ausschreibungen können Auktionen für den Fall von verdeckten Angeboten grundsätzlich durch eine Vorzeichenumkehr auf den Ausschreibungsfall transformiert werden (Römhild 1997, S. 23-24). Dabei wird nachfolgend von Angeboten gesprochen, um zu verdeutlichen, dass im Rahmen eines Ausschreibungsverfahrens eine definierte Leistung zu einem bestimmten Preis angeboten wird und nicht lediglich ein Preis für ein Gut geboten wird. Bei Ausschreibungen von Umweltleistungen erfolgt demzufolge nicht die Offenbarung einer Zahlungsbereitschaft seitens der Bieter, sondern die Angabe, zu welchem Preis ein Anbieter bereit ist, eine Umweltleistung zu erbringen (Cason und Gangadharan 2003, S. 1-2; Stoneham 2003, S. 485-490).

Wie bei einer Auktion werden die Regeln für das Ausschreibungsverfahren durch die Vergabestelle vor Beginn der Ausschreibung festgelegt, den potentiellen Anbietern bekannt gegeben und können nachträglich nicht mehr geändert werden (Leitzinger 1988, S. 74). Somit ist eine Ausschreibung für öffentliche Güter - wie ökologische Leistungen der Landwirtschaft - ebenfalls als ein Marktmechanismus zu definieren, durch dessen Einsatz innerhalb eindeutig festgelegter Regeln und auf der Grundlage von Angeboten teilnehmender Anbieter (Landwirte), Güter (Umweltleistungen) nachgefragt und Preise ermittelt werden (Latacz-Lohmann und Van der Hamsvoort 1997, S. 416-417). Eine wesentliche Besonderheit ist demzufolge darin zu sehen, dass hier grundsätzlich ein zweidimensionales Zuschlagskriterium in Form einer ökonomischen und einer ökologischen Angebotskomponente Berücksichtigung findet.

Die Ziele eines Ausschreibungsverfahrens für Umweltleistungen sind eng verbunden mit der Überwindung der Probleme einer Einheitsprämie, wie sie derzeit in Agrarumweltprogrammen verankert ist und können darauf aufbauend dargelegt werden.

Bei einer Einheitsprämie ist das zentrale Problemfeld, dass alle teilnehmenden Landwirte unabhängig von ihren individuellen Kosten der Leistungserbringung eine einheitliche Zahlung erhalten. Neben den grundsätzlich mit einer derartigen Honorierung verbundenen Problemen kommt es zudem bedingt durch eine asymmetrische Informationsverteilung zu Lasten der Agrarverwaltung $\mathrm{zu}$ der Situation, dass die Festlegung der Einheitsprämie in Unkenntnis der tatsächlichen 
Kosten der landwirtschaftlichen Betriebe erfolgt (Latacz-Lohmann und Van der Hamsvoort 1997, S. 407-408; Hart und Latacz-Lohmann 2005, S. 76).

Es ist ersichtlich, dass ein solches Vorgehen aus ökonomischer Sicht zu kritisieren ist und Ausschreibungen von Umweltleistungen seitens der Vergabestelle die folgenden drei Ziele fokussieren:

I. Kostenminimierung / Nutzenmaximierung: Durch eine Ausschreibung soll entweder ein bestimmter ökologischer Nutzen mit einem minimalen öffentlichen Finanzmitteleinsatz erreicht oder mit einem gegebenen öffentlichen Budget ein möglichst hoher ökologischer Nutzen erzielt werden.

II. Effizienz: Die Zuschlagserteilung soll an den Landwirt gehen, der die ausgeschriebene Umweltleistung zu den geringsten Kosten erbringen kann.

III. Informationsgewinnung: Durch auf der individuellen Kostensituation beruhende Angebote ist es möglich, die bestehende Informationsasymmetrie zu minimieren und Informationen über die Kosten der Landwirte zu erlangen.

Zusammenfassend ist zu erkennen, dass innerhalb einer Ausschreibung grundsätzlich divergierende Zielsetzungen und Anforderungen der handelnden Akteure vorhanden sind. Das Ziel der Vergabestelle ist es, ökologische Leistungen durch die Wahl eines geeigneten Ausschreibungsdesigns zu einem möglichst niedrigen Preis einzukaufen, wohingegen für die Landwirte der Anreiz besteht, durch die Wahl einer geeigneten Bietstrategie ökologische Leistungen zu einem möglichst hohen Preis zu verkaufen (Leitzinger 1988, S. 73-74; Holm-Müller et al. 2002, S. 114).

\subsubsection{Eingutausschreibungen}

Analog zu den Eingutauktionen werden nun vier Ausgestaltungsmöglichkeiten von Ausschreibungen abgeleitet, jeweils anhand ihrer grundlegenden Merkmale beschrieben und abschließend im Hinblick auf eine praktischen Einsetzbarkeit im hier relevanten Kontext bewertet.

\section{Englische Ausschreibung}

Bei einer Englischen Ausschreibung werden die Angebote öffentlich abgegeben, wobei der Angebotspreis beginnend von einem hohen Ausgangspreis solange sukzessive verringert wird, bis kein Bieter mehr bereit ist den aktuellen Angebotspreis zu unterbieten. Der Zuschlag geht an den Bieter mit der geringsten 
Forderung, welche entsprechend ausgezahlt wird. Die Bieter haben somit die Möglichkeit, wiederholt Angebote abzugeben und dabei Informationen über andere Bieter und deren Angebote zu berücksichtigen.

Die praktischen Einsatzmöglichkeiten einer Englischen Ausschreibung zur Honorierung von Umweltleistungen sind kritisch einzuschätzen, weil zum einen in der Praxis ein räumliches Zusammentreffen der Bieter nicht zu gewährleisten ist und zum anderen die Bieter die Preisinformationen der anderen Bieter durch die offene Angebotsabgabe unmittelbar berücksichtigen können. Letzteres eröffnet die Gefahr, dass die Preisentscheidung nicht (ausschließlich) auf den individuellen Kosten basiert. Neben den daraus zu erwartenden negativen Auswirkungen auf die Effizienzund Kostenminimierungsziele ist zudem nur eine eingeschränkte Informationsgewinnung hinsichtlich der tatsächlichen Kostensituation der Landwirte zu erwarten. Demzufolge muss eine Englische Ausschreibung ökologischer Leistungen der Landwirtschaft als nicht zweckmäßig beurteilt werden.

\section{Holländische Ausschreibung}

Im Gegensatz zur Englischen Ausschreibung beginnt eine Holländische Ausschreibung mit einem geringen Angebotspreis, welcher solange sukzessive erhöht wird, bis der erste Bieter ein Angebot abgibt und damit den aktuellen Preis akzeptiert. Dieser Bieter erhält den Zuschlag und die entsprechende Zahlung. Folglich besteht trotz der offenen Angebotsabgabe für die Bieter keine Möglichkeit mehrfache Angebote abzugeben, da mit einem ersten Angebot die Ausschreibung beendet ist.

Die praktischen Einsatzmöglichkeiten eines Holländischen Ausschreibungsverfahrens sind grundsätzlich positiver als bei einer Englischen Ausschreibung einzuschätzen. Vor allem erfüllt sie das Ziel, dass sich alle Bieter an ihrer individuellen Kostenstruktur orientieren und der Bieter mit den geringsten Kosten den Zuschlag bekommt. Kritisch ist eine Holländische Ausschreibung jedoch im Blick auf die Informationsgewinnung einzuschätzen, da lediglich ein Angebot abgegeben wird und Rückschlüsse nur zu den Kosten des erfolgreichen Bieters getroffen werden können. ${ }^{89}$ Die Holländische Ausschreibung ist somit je nach

\footnotetext{
${ }^{89}$ Es ist selbstverständlich abzuleiten, dass die übrigen Bieter über höhere Kosten verfügen, was jedoch nicht als entscheidender Erkenntnisgewinn aufgefasst werden kann.
} 
Ausprägung der Zielsetzung für den Bereich ökologischer Leistungen als bedingt geeignet einzuschätzen.

\section{Niedrigstpreis-Ausschreibung}

Bei einer Niedrigstpreis-Ausschreibung werden die Angebote verdeckt abgegeben und der Zuschlag wird dem Bieter erteilt, der bereit ist die ausgeschriebene Leistung zu dem niedrigsten Preis zu erbringen. Hierbei ist es für jeden Bieter nur einmal möglich ein Angebot abzugeben und der erfolgreiche Bieter erhält den Zuschlag zu den von ihm angebotenen Konditionen. Die Niedrigstpreis-Ausschreibung stellt die gebräuchlichste Form des Ausschreibungsverfahrens dar und ist mit der Ausschreibung an sich gleichzusetzen.

Der Einsatz einer Niedrigstpreis-Ausschreibung für ökologische Leistungen ist durchweg als positiv zu beurteilen, da alle drei direkten Ziele realisiert werden können. Die Angebotsabgabe erfolgt auf der Grundlage der spezifischen Kostensituation und der Bieter mit den geringsten Kosten erhält den Zuschlag. Zudem gibt jeder interessierte Bieter ein individuelles Angebot ab, was für die Vergabestelle die Möglichkeit schafft, umfangreiche Informationen von allen Bietern zu generieren.

\section{Zweitpreis-Ausschreibung}

Ebenso wie bei der Niedrigstpreis-Ausschreibung geben die Bieter ihre Gebote bei einer Zweitpreis-Ausschreibung verdeckt und einmalig ab. Der Bieter, der bereit ist die Leistung zu dem niedrigsten Preis zu erbringen, erhält auch hier den Zuschlag, jedoch sieht die Zweitpreis-Ausschreibung vor, dass der erfolgreiche Bieter nicht seinen eigenen Preis, sondern den des zweitniedrigsten Angebots ausgezahlt bekommt.

Bis auf die letztliche Preisfestlegung ist die Zweitpreis-Ausschreibung mit der Niedrigstpreis-Ausschreibung als grundsätzlich identisch zu bewerten. Die Angebotsabgabe erfolgt ebenfalls auf der Grundlage der einzelwirtschaftlichen Kosten und der Bieter mit den geringsten Kosten erhält den Zuschlag. Ebenso ist durch die notwendige Angebotsabgabe jedes Bieters eine umfassende Informationsgewinnung möglich. Die theoretisch überzeugende Preisfestlegung in Höhe des zweitniedrigsten Preises ist in der Praxis jedoch mit Problemen behaftet, da 
sie für die Bieter mitunter schwer nachvollziehbar ist und als ungerecht empfunden werden kann.

Zusammenfassend zeigt sich für die Eingutausschreibungen zur Honorierung ökologischer Leistungen innerhalb dieser Arbeit und der im Zuge dessen verfolgten Zielsetzungen (Kostenminimierung, Effizienz, Informationsgewinnung), dass ausschließlich die Ausschreibungsvarianten mit einer verdeckten Angebotsabgabe in Betracht kommen.

Hierbei wurde bislang jedoch lediglich die Option einer Eingutausschreibung betrachtet, was für den praktischen Einsatz in der Regel zu kurz greift, so dass im folgenden Abschnitt analog $\mathrm{zu}$ den Mehrgüterauktionen die Mehrgüterausschreibungen einbezogen werden.

\subsubsection{Mehrgüterausschreibungen}

Für eine realitätsnahe Betrachtung und zur Charakterisierung des im Rahmen dieser Arbeit gewählten Ausschreibungsdesigns ist es notwendig, auch die folgenden Optionen von Mehrgüterausschreibungen hinsichtlich ihrer praktischen Einsetzbarkeit für die Honorierung ökologischer Leistungen zu diskutieren.

\section{Sequentielle Ausschreibung}

Die Ausgestaltungsoption einer sequentiellen Ausschreibung stellt den Fall dar, bei dem innerhalb eines eng begrenzten Zeitraums mehrere identische oder nicht identische Güter gehandelt werden. Für den Fall, dass jeweils nur ein Gut ausgeschrieben wird, kann faktisch von einer Aneinanderreihung von Eingutausschreibungen gesprochen werden. Bei der zeitgleichen Ausschreibung mehrerer Güter liegt eine wiederholte Mehrgüterausschreibung vor.

Die praktische Relevanz einer sequentiellen Ausschreibung für Umweltleistungen der Landwirtschaft ist vor allem aus zwei Gründen nicht gegeben. Zum einen erfolgen die mehrfachen Ausschreibungen zeitlich sehr eng nacheinander und zum anderen kommt immer ein identisches Ausschreibungsdesign zur Anwendung. Hierbei ist insbesondere die Nutzung eines unveränderten Ausschreibungsdesigns vor dem Hintergrund des Lernens seitens der Bieter und der Gefahr daraus resultierender Preisabsprachen in der Praxis nicht sinnvoll. 


\section{Mengentender („Einheitsprämie“)}

Die Mehrgüterausschreibung eines Mengentenders besagt, dass mehrere identische Güter nachgefragt werden und seitens der Vergabestelle vorab ein fester Preis für diese Güter gesetzt wird. Für die Landwirte besteht lediglich die Mengenentscheidung, ob und wenn ja wie viele Güter sie zu dem gegebenen Preis anbieten.

Folglich stellt ein Mengentender in letzter Konsequenz den zu einer derzeit in Agrarumweltprogrammen eingesetzten Einheitsprämie analogen Fall dar und ist hier als Option für ökologische Leistungen ungeeignet.

\section{Simultane Ausschreibungen mit Preis-Mengen-Angeboten}

Auch für die Mehrgüterausschreibungen sind drei Ausgestaltungsmöglichkeiten von simultanen Ausschreibungen mit Preis-Mengen-Angeboten zu unterscheiden. Innerhalb dieser einmalig durchgeführten Ausschreibung werden von der Vergabestelle mehrere identische Güter nachgefragt und das Angebot für ökologische Leistungen umfasst sowohl eine Mengen- als auch eine Preiskomponente. Im Zuge dessen können Landwirte mehrere Angebote abgeben, welche nach Ablauf der Angebotsfrist hinsichtlich des Angebotspreises bewertet und in eine Rangfolge gebracht werden. Die Zuschlagserteilung erfolgt jeweils beginnend mit dem niedrigsten Preis »von unten nach oben«.

Alle drei Optionen einer simultanen Ausschreibung mit Preis-Mengen-Angeboten beruhen auf diesem konzeptionellen Gerüst; zu unterscheiden sind jedoch die jeweiligen Preisgestaltungsmöglichkeiten.

\section{IIIa. Einheitspreis-Ausschreibung}

Bei einer Einheitspreis-Ausschreibung ist für die Zuschlagserteilung der individuelle Angebotspreis eines Landwirts entscheidungsrelevant. Nach der Zuschlagserteilung erhalten die Landwirte jedoch für alle angenommenen Angebote eine Honorierung in Höhe eines einheitlichen Preises, welcher in der Regel dem höchsten noch berücksichtigten oder dem niedrigsten nicht mehr berücksichtigten Angebotspreis je Einheit entspricht. Auf den ersten Blick mag die Einheitspreis-Ausschreibung Analogien zu einer Einheitsprämie aufweisen, da alle Landwirte eine einheitliche Honorierung erhalten. Von der Einheitsprämie unterscheidet sich dieser Ansatz 
jedoch fundamental dadurch, dass die Angebotspreise der Landwirte die Entscheidungsgrundlage darstellen.

Für ein einmaliges Ausschreibungsverfahren ist eine Einheitspreis-Ausschreibung insofern als positiv einzuschätzen, dass die individuellen Angebote relevant sind und entsprechende Informationen aller Bieter erfasst werden können. Es erfolgt jedoch letztlich keine ausschließlich an den jeweiligen Kosten orientierte Zahlung, was zu Effizienzeinbußen führen kann.

Für mehr als ein identisches Ausschreibungsverfahren ist eine EinheitspreisAusschreibung als ungeeignet zu bewerten, da die Anbieter nun nach Abschluss der Ausschreibungsrunde aus der einheitlichen Zahlung lernen können und zu erwarten ist, dass diese Informationen bei kommenden Ausschreibungen Berücksichtigung finden. Dies hätte ein Abweichen von der individuellen Preiskalkulation mit entsprechenden Effizienz- und Informationseinbußen zur Folge.

\section{IIIb. Preisdiskriminierende Ausschreibung}

Die preisdiskriminierende Ausschreibung ist bezüglich der Preisfestsetzung das konzeptionelle Gegenteil zu der Einheitspreis-Ausschreibung. Der Ablauf bis zur Zuschlagserteilung ist identisch, doch letztlich erhalten die Landwirte eine finanzielle Honorierung in Höhe des ihrem individuellen Angebot entsprechenden Angebotspreises.

Für die Vergabestelle besteht demzufolge die Möglichkeit, eine identische ökologische Leistung zu unterschiedlichen Preisen einzukaufen und Informationen über die Kosten aller Anbieter zu erhalten. Darüber hinaus erfolgt eine kostenorientierte Honorierung und für den Fall, dass die jeweiligen Zuschlagsgrenzen nach Abschluss der Zuschlagserteilung seitens der Vergabestelle nicht öffentlich gemacht werden, ist für die wiederholte Nutzung ebenso eine entsprechend positive Zielerreichung $\mathrm{zu}$ erwarten. Daher ist die preisdiskriminierende Mehrgüterausschreibung sowohl für den einmaligen als auch den mehrmaligen praktischen Einsatz zur Honorierung von Umweltleistungen als sehr gut geeignet einzuschätzen.

\section{IIIc. Zweitpreis-Mehrgüterausschreibung}

Das Gegenstück zu der Zweitpreis-Ausschreibung für ein Gut stellt für mehr als ein Gut die Zweitpreis-Mehrgüterausschreibung dar. Auch hier können Landwirte 
verschiedene Angebote für identische ökologische Güter abgeben, welche bei der Zuschlagserteilung anhand der individuellen Angebotspreise bewertet werden. Die Festlegung der Zahlungen an die Landwirte erfolgt, indem die erfolgreichen Landwirte nicht die ihren Angeboten entsprechenden Preise, sondern die Preise der Angebote erhalten, die gerade noch angenommen worden wären, wenn die erfolgreichen Bieter nicht an der Ausschreibung teilgenommen hätten.

Ebenso wie die Zweitpreis-Ausschreibung ist die ZweitpreisMehrgüterausschreibung derzeit als theoretisches Konstrukt einzuschätzen und für den praktischen Einsatz zur Honorierung ökologischer Leistungen der Landwirtschaft nicht geeignet.

\section{Simultane mehrperiodige Ausschreibung}

Die simultane mehrperiodige Ausschreibung ist eine Kombination der Englischen Ausschreibung und der Niedrigstpreis-Ausschreibung, innerhalb derer zeitlich begrenzte Ausschreibungsrunden nacheinander durchgeführt werden, bei denen die Landwirte Angebote für mehrere Güter abgeben können. Die eingegangenen Angebote werden nach Abschluss einer Ausschreibungsrunde in eine Rangfolge gebracht und der jeweils aktuelle Stand der Angebote bekannt gemacht. Die Anbieter können nun in sich anschließenden Ausschreibungsrunden darauf reagieren und die jeweiligen Niedrigstgebote unterbieten, bis sich kein Anbieter mehr findet der ein niedrigeres Gebot abgibt.

Demzufolge ist eine derart ausgestaltete Ausschreibung für den Einsatz zur Honorierung von Umweltleistungen nicht praktikabel, da sowohl Lerneffekte als auch ein Abweichen von der individuellen Preiskalkulation Effizienzeinbußen erwarten lassen. Zudem ist die Notwendigkeit eines räumlichen Zusammentreffens der Anbieter als nicht umsetzbar einzuschätzen.

Zusammenfassend zeigt sich, dass mit Blick auf eine praktische Umsetzung ausschließlich Mehrgüterausschreibungen mit Preis-Mengen-Angeboten und einer verdeckten Angebotsabgabe geeignet sind, da so vermieden werden kann, dass die Informationen konkurrierender Anbieter Berücksichtigung finden können. Darüber hinaus sind die beiden relevanten Ausschreibungstypen (EinheitspreisAusschreibung und preisdiskriminierende Ausschreibung) insbesondere im Hinblick auf die durch sie induzierten strategischen Anreize und das demzufolge zu 
erwartende Bieterverhalten der Landwirte erneut aufzugreifen und im Detail zu diskutieren.

Der Hauptunterschied zwischen der Einheitspreis-Ausschreibung und der preisdiskriminierenden Ausschreibung liegt darin, dass das individuelle Angebot eines Landwirts im Rahmen einer Einheitspreis-Ausschreibung ausschließlich die Wahrscheinlichkeit einer Zuschlagserteilung und nicht unmittelbar die Höhe der zu erhaltenden Zahlung beeinflusst, wodurch keine Anreize für ein Überbieten in Form eines die jeweiligen Opportunitätskosten übersteigenden Angebotspreises vorhanden sind. Die theoretisch optimale Bietstrategie ist somit die Offenbarung der exakten Opportunitätskosten, da durch einen höheren Angebotspreis die Wahrscheinlichkeit der Zuschlagserteilung reduziert wird und es für den Fall eines geringeren Angebotspreises zu einer Unterkompensation der Kosten der Erbringung einer Umweltmaßnahme kommt.

Demgegenüber ist die Situation innerhalb einer preisdiskriminierenden Ausschreibung dadurch gekennzeichnet, dass sowohl die Wahrscheinlichkeit der Zuschlagserteilung als auch die Höhe der zu erhaltenden Zahlung durch die individuellen Angebote der Landwirte bestimmt werden. Daraus folgt der Anreiz, die tatsächlichen Opportunitätskosten zu überbieten und im Zuge dessen eine zu hohe Zahlung zu realisieren.

Auf der Grundlage dieser vor allem theoretisch geprägten und für die einmalige Durchführung eines Ausschreibungsverfahrens gültigen Argumentation liegt demnach der Schluss nahe, die Einheitspreis-Ausschreibung einer preisdiskriminierenden Zuschlagserteilung mit dem Ziel einer kostenminimierenden und effizienten Umsetzung vorzuziehen. Dem stehen jedoch einige entscheidende praktische Überlegungen gegenüber, welche nachfolgend einbezogen werden.

Ein zentraler Aspekt ist darin $\mathrm{zu}$ sehen, dass Landwirte mit geringen Anpassungskosten überproportional von einer hohen einheitlichen Zahlung profitieren können und es im Rahmen einer Einheitspreis-Ausschreibung zu mitunter massiven Überkompensationen kommen kann. Damit einhergehen Praktikabilitätsund Akzeptanzprobleme, da zu erwarten ist, dass ein derartiges Vorgehen aus Sicht der Landwirte überwiegend als nicht gerecht und zu kompliziert empfunden wird. Zudem ist eine Einheitspreis-Ausschreibung für ein wiederholtes identisches Ausschreibungsverfahren dahingehend als ungeeignet anzusehen, dass die Anbieter nach Abschluss der Ausschreibungsrunde aus der einheitlichen Zahlung lernen und 
diese Informationen bei kommenden Ausschreibungen Berücksichtigung finden können. Dies hätte ein Abweichen von der individuellen Preiskalkulation mit entsprechenden Effizienz- und Informationseinbußen zur Folge.

Darüber hinaus ist die Einflussmöglichkeit der Vergabestelle anzuführen, mit Hilfe einer Budgetrestriktion oder eines Reservationspreises eine Konkurrenzsituation zu erzeugen und im Rahmen einer wiederholten preisdiskriminierenden Zuschlagserteilung einem Überbieten der individuellen Opportunitätskosten entgegenzuwirken, da mit einem steigenden Angebotspreis in der Regel die Wahrscheinlichkeit einer Zuschlagserteilung abnimmt. Zudem sind bei einer wiederholten Durchführung eines Ausschreibungsverfahrens die Vorteile der Aufrechterhaltung einer an den individuellen Kosten orientierten Preisbildung ausschließlich bei einer Preisdiskriminierung zu erwarten. Abschließend ist daher die Konzeption einer preisdiskriminierenden Ausschreibung einer EinheitspreisAusschreibung vor dem Hintergrund der hier angestrebten praktischen Umsetzung vorzuziehen. ${ }^{90}$

\subsubsection{Weitere Ausgestaltungsmöglichkeiten}

Über die oben angeführten hinaus, bestehen im hier relevanten Kontext weitere grundsätzliche Ausgestaltungsmöglichkeiten von Ausschreibungen. Diese beziehen sich sowohl auf die regionale Abgrenzung als auch auf die Wahl des Zuschlagskriteriums und werden im Folgenden einbezogen.

\section{Ia. Ausschreibungsverfahren mit einer einheitlichen Ausschlussgrenze}

Bei einem Ausschreibungsverfahren mit einer einheitlichen Ausschlussgrenze werden alle teilnahmeberechtigten Landwirte im Geltungsbereich des Ausschreibungsverfahrens identisch behandelt und sie konkurrieren allesamt gegeneinander um die Zuschlagserteilung. Um eine Konkurrenzsituation sicherzustellen, wird den Landwirten bis zum Zeitpunkt der Zuschlagserteilung mitgeteilt, dass ausschließlich die Angebote einen Zuschlag erhalten werden, welche

\footnotetext{
${ }^{90}$ Eine zusammenfassende Einschätzung, die unter anderem grundsätzlich auch von Latacz-Lohmann und Van der Hamsvoort, Cason und Gangadharan und Stoneham et al. vertreten wird (LataczLohmann und Van der Hamsvoort 1997, S. 409; Cason und Gangadharan 2003, S. 18-20; Stoneham et al. 2003, S. 496).
} 
unterhalb einer den Landwirten nicht bekannten Ausschlussgrenze liegen (LataczLohmann 1993, S. 354-355).

\section{Ohne Budgetrestriktion}

Für den Fall, dass staatlicherseits keine Budgetrestriktion vorliegt, werden entgegen der ursprünglichen Ankündigung alle eingegangenen Angebote angenommen. Dies hat den Vorteil, dass eine große Anzahl von Flächen in ein Programm genommen und ein möglichst hoher ökologischer Nutzen bei einer gleichzeitigen individuellen Preiskalkulation unter Konkurrenzbedingungen erreicht werden kann.

Dieser Vorteil ist jedoch nur bei einer einmalig durchgeführten Ausschreibung zu realisieren. Ab einer zweiten identischen Ausschreibungsrunde ist zu erwarten, dass die Landwirte trotz der Bekanntgabe einer Ausschlussgrenze dieser keinen Glauben schenken und in der Erwartung einer erneuten Annahme aller Angebote höhere Angebotspreise abgeben werden.

\section{Mit Budgetrestriktion}

Unterliegt die staatliche Vergabestelle bei der Zuschlagserteilung einer Budgetrestriktion, so wird vorab eine den Landwirten nicht bekannte Ausschlussgrenze festgelegt. Dabei erfolgt eine Zuschlagserteilung in der Reihenfolge der Angebote bis zum Erreichen der Ausschlussgrenze oder bis zur Ausschöpfung des zur Verfügung stehenden Budgets. Folglich können der ursprünglichen Bekanntmachung entsprechend in der Regel nicht alle eingegangenen Angebote Berücksichtigung finden, was auch über mehrere Ausschreibungsrunden hinaus eine kostenorientierte Preiskalkulation unter Konkurrenzbedingungen erwarten lässt. Dies jedoch mit der Einschränkung, dass nicht alle Flächen in ein Programm genommen werden und gegebenenfalls naturschutzfachlich interessante Angebote keine Berücksichtigung finden können.

\section{Ib. Ausschreibungsverfahren mit einer regionalisierten Ausschlussgrenze}

Entgegen der vorherigen Ausgestaltung wird nun die Möglichkeit betrachtet, innerhalb des Geltungsbereichs eines Ausschreibungsverfahrens eine regionale Differenzierung der Ausschlussgrenze vorzunehmen. Es können wiederum alle Landwirte ein Angebot abgeben, wobei die Zuschlagserteilung regional unterschiedlich erfolgt und im Rahmen der Bekanntmachung des 
Ausschreibungsverfahrens auf diese regionalen Abgrenzungen ebenso hingewiesen wird, wie darauf, dass ausschließlich Angebote akzeptiert werden, die unter der jeweiligen - den Landwirten nicht bekannten - regionalen Ausschlussgrenze liegen (Latacz-Lohmann 1993, S. 354-355).

Vorteile sind dahingehend zu sehen, dass eine an den ökologischen Gegebenheiten orientierte Regionalisierung dazu führt, dass sowohl ökologisch als auch ökonomisch vergleichbare Betriebe miteinander konkurrieren und eine Verzerrung der Wettbewerbssituation durch übermäßige Kostenunterschiede vermieden werden kann. Allerdings ist $\mathrm{zu}$ beachten, dass keine $\mathrm{zu}$ enge regionale Abgrenzung vorgenommen wird und durch eine ausreichende Anzahl und Heterogenität potentieller Anbieter eine Preiskalkulation unter Konkurrenzbedingungen erfolgen kann. Hinsichtlich der Existenz einer Budgetbeschränkung und ihrer Auswirkungen sind für die jeweiligen Regionen die analogen Schlüsse wie für den Fall mit einer einheitlichen Ausschlussgrenze zu ziehen.

\section{Wahl des Zuschlagskriteriums}

Die letzte Ausgestaltungsmöglichkeit behandelt die Frage des Kriteriums, nach dem die formal korrekt und fristgerecht eingegangenen Angebote seitens der Vergabestelle bewertet werden. Ein essentieller Bestandteil des Zuschlagskriteriums ist der Angebotspreis je Einheit (in der Regel je Hektar) der angebotenen Fläche. Bei ökologisch identischen oder nahezu identischen Umweltleistungen ist die ausschließliche Berücksichtigung des Angebotspreises bei der Zuschlagserteilung angemessen, da der ökologische Nutzen implizit durch die Leistungsbeschreibung Eingang findet und eine Vergleichbarkeit gegeben ist.

Darüber hinaus besteht die Möglichkeit, zusätzlich zu der ökonomischen Komponente, eine ökologische Komponente beispielsweise durch einen Umweltindex oder die Vergabe von Ökopunkten in die Zuschlagserteilung einzubeziehen und eine kombinierte Bewertung der Angebote vorzunehmen (Knauer 1993, S. 228-230; Plankl 1999, S. 174; Bertke 2005, S. 5-8). Hierbei erfolgt das Ranking der eingegangenen Angebote gemäß eines Katalogs von Kriterien, welcher neben dem Angebotspreis auch ökologische Merkmale - wie den naturschutzfachlichen Wert der Fläche oder deren Betroffenheit von einem Schutzgebiet - berücksichtigt. Diese kombinierte Bewertung ist vor allem 
zweckmäßig, wenn innerhalb eines Ausschreibungsverfahrens eine große Anzahl potentiell anzubietender Flächen in ökologisch verschiedenen Regionen mit einem bestimmten zu erreichenden Umweltziel (z.B. Erosionsschutz) ausgeschrieben werden und je nach Lage und Art der Fläche unterschiedlichste Einflüsse auf die Zielerreichung zu erwarten sind. ${ }^{91}$

\subsubsection{Ausschreibungsdesign im Rahmen des Forschungsprojekts}

Unter Rückgriff auf die bisherigen Ausführungen wird abschließend das innerhalb dieser Arbeit gewählte Ausschreibungsdesign in seiner konzeptionellen Ausgestaltung zusammenfassend vorgestellt.

Es wurde für das Forschungsprojekt und die empirischen Untersuchungen eine simultane Mehrgüterausschreibung mit Preis-Mengen-Angeboten und einer preisdiskriminierenden Zuschlagserteilung gewählt und im Rahmen von zwei aufeinander folgenden Ausschreibungsrunden praktisch erprobt. Dieses auf der Standardausschreibung einer Niedrigstpreis-Ausschreibung beruhende Verfahren beinhaltet durch eine verdeckte Angebotsabgabe die Möglichkeit, ökologische Leistungen zu unterschiedlichen Preisen nachzufragen und im Zuge dessen einen differenzierten und an den individuellen Kosten der Landwirte orientierten Einsatz finanzieller Mittel zu verfolgen.

Hinsichtlich der Zuschlagserteilung wurde das Vorgehen einer kombinierten Bewertung gewählt. Aus ökonomischer Sicht wurde der Angebotspreis pro Hektar herangezogen und als ökologisches Kriterium fungierte die Einstufung der angebotenen ökologischen Leistungen in eine von drei Qualitätsabstufungen. ${ }^{92}$

Die regionale Abgrenzung entsprach einer einheitlichen Ausschlussgrenze mit Budgetrestriktion. Demzufolge konkurrierten alle Landwirte in der Modellregion mit ihren teilnahmeberechtigten landwirtschaftlichen Flächen gegeneinander um die Zuschlagserteilung.

Damit wurde das Ziel verfolgt, eine möglichst hohe Anzahl an Landwirten zu erreichen, umfassende Informationen zu generieren und zudem eine größtmögliche Konkurrenzsituation zu erzeugen.

\footnotetext{
${ }^{91}$ Ein zentrales Beispiel für den praktischen Einsatz eines Umweltindikators ist der im Conservation Reserve Program in den USA verwendete Environmental Benefits Index. Dieser wird in Abschnitt 5.3.3 im Rahmen der Betrachtung des Conservation Reserve Program ausführlich behandelt.

92 Auf das ökologische Kriterium wird im Rahmen der praktischen Umsetzung der Ausschreibungsverfahren in Kapitel Sieben näher eingegangen.
} 
Die Ausgestaltung der Ausschreibungsunterlagen und die Abwicklung der Ausschreibungen wurden in Anlehnung an die Verdingungsverordnung für Bauleistungen (VOB) durchgeführt. ${ }^{93}$

\subsection{Conservation Reserve Program}

Sein langjähriger praktischer Einsatz und seine Bedeutung als größtes Umweltschutzprogramm des U.S. Agrarministeriums (USDA) ${ }^{94}$ begründen die Sonderstellung des im Folgenden ausführlich betrachteten Conservation Reserve Program (CRP) als marktorientierten Ansatz zur Honorierung von Umweltleistungen.

\subsubsection{Entwicklung, Ziele und Grundlagen}

Das CRP wurde vom USDA 1985 innerhalb des Food Security Act mit dem Ziel der Verhinderung einer fortschreitenden Erosion landwirtschaftlich genutzter Ackerflächen eingeführt (Reichelderfer und Boggess 1988, S. 1; Anderson 1995, S. 4). Es handelt sich somit beim CRP nicht um ein Agrarumweltprogramm im Charakter der Verordnung VO (EWG) Nr. 2078/92, sondern um eine ökologisch motivierte Stilllegung von landwirtschaftlichen Flächen (Mello et al. 2002, S. 85).

Das CRP ist ein auf einem Ausschreibungsverfahren basierendes Konzept zur Bewertung und Honorierung ökologischer Leistungen der Landwirtschaft, mit dem definierte Konservierungsmaßnahmen auf Ackerflächen von Landwirten angeboten und staatlicherseits hinsichtlich ihrer ökologischen und ökonomischen Aspekte bewertet werden können (Plankl 1999, S. 165-166).

Die zu honorierenden ökologischen Leistungen sind die Durchführung von Maßnahmen zur Erosionsverringerung und damit der Dezimierung ökologischer und ökonomischer Schäden beispielsweise durch die Erosion fruchtbaren Ackerlands oder die Versandung von Seen und Flüssen.

\footnotetext{
${ }^{93}$ Die Durchführung einer Ausschreibung ist hierbei den in $\S 30$ des Haushaltsgrundsätzegesetzes (HGrG) getroffenen Bestimmungen unterworfen, wobei mit der öffentlichen Ausschreibung, der beschränkten Ausschreibung und der freihändigen Vergabe drei Vergabearten für öffentliche Aufträge vorgesehen sind. Für weitergehende Ausführungen sei an dieser Stelle auf die Arbeit von Hespelt (2005) verwiesen.

${ }^{94}$ United States Department of Agriculture.
} 
Die Notwendigkeit eines zunehmenden Erosionsschutzes ist dabei nicht neu gewesen und sowohl die klimatischen Einflüsse als auch eine unangemessene Bewirtschaftung landwirtschaftlicher Flächen haben dazu geführt, dass es in den Staaten des Mittleren Westens zu einer Erosionsrate von bis zu 152 Tonnen pro Jahr und Hektar gekommen ist (Blackburn et al. 1991, S. 27). So wurden erste Erosionsschutzbemühungen maßgeblich durch die als „Dust Bowl“ bezeichneten Sandstürme von 1934 und 1935 hervorgerufen, bei denen nach einer andauernden Trockenheit große Mengen Staub aufgewirbelt wurden. Daraufhin wurde 1935 der Soil and Water Conservation Act beschlossen (Laycock 1991, S. 2; Brehm 2000, S. 4). 1956 wurde mit dem Soil Bank ein neues Program aufgelegt, was die Reduktion von Überschüssen mit Umweltschutzzielen kombinierte. Bis zum Zeitpunkt des Auslaufens der darin geschlossenen langfristigen Verträge im Jahr 1972 konnten rund 29 Millionen acres ${ }^{95}$ stillgelegt werden. Dennoch dauerte es bis zur Einführung des CRP im Jahr 1985, dass ein ähnliches Programm erneut angeboten wurde (Smith 2003, S. 2-3; USGAO 1993, S. 2).

Die mit der Einführung des CRP offiziell verfolgten Ziele lassen sich auf die folgenden Stichpunkte zusammenfassen (Reichelderfer und Boggess 1988, S. 1; Feather et al. 1999, S. 5):

Einschränkung der durch Wind und Wasser verursachten Bodenerosion,

$>$ Langfristige Sicherung der Fähigkeit zur Erzeugung von Lebensmitteln und Rohstoffen,

$>$ Reduzierung der Sedimentation,

$>$ Verbesserung der Wasserqualität,

$>$ Verbesserung der Lebensräume für Fische und wildlebende Tiere,

$>$ Verringerung von Produktionsüberschüssen,

Einkommensunterstützungen für Landwirte.

Seit 1985 wurde das CRP durch nachfolgende Farm Bills regelmäßig bestätigt und erneut aufgelegt. ${ }^{96}$ Im Verlauf dieser Neuauflagen ist es sowohl im Hinblick auf die

\footnotetext{
${ }^{95}$ Ein acre entspricht 0,4047 Hektar.

${ }^{96}$ Im Einzelnen durch den Food, Agriculture, Conservation and Trade Act (FACT) aus dem Jahr 1990, den 1996er Federal Agricultural Improvement and Reform Act (FAIR) und 2002 durch die jüngste Farm Bill, den Farm Security and Rural Investment Act. Durch letzteren ist der Einsatz des CRP bis einschließlich 2007 sichergestellt (Smith 2003, S. 2; USDA 2003b).
} 
ökologische Ausrichtung als auch methodisch zu mehr oder weniger umfassenden Anpassungen gekommen, von denen die bedeutendsten nun kurz betrachtet werden. Das ursprünglich angestrebte Umweltziel war, bis 1990 zwischen 40 und 45 Millionen acres Ackerland in das Programm zu nehmen; eine Fläche, die rund $10 \%$ der gesamten Ackerfläche in den USA entsprochen hätte (Shoemaker 1989, S. 131). Für die folgende Periode bis einschließlich 1996 wurde dieses Ziel anfangs fortgeschrieben, dann jedoch auf maximal 38 Millionen acres reduziert (Osborn et al. 1995, S. 4). Mit dem anschließenden Farm Act aus dem Jahr 1996 wurde die Grenze der maximal in das Programm einzubeziehenden Fläche erneut reduziert und bis zum Jahr 2002 auf 36,4 Millionen acres festgelegt (Osborn 1997, S. 286). Eine Trendwende wird durch die derzeit aktuelle Farm Bill aus dem Jahr 2002 deutlich, innerhalb derer die Obergrenze auf 39,2 Millionen acres angehoben wurde, was ungefähr 15,9 Millionen Hektar und rund der 8,6 \% der gesamten Ackerfläche in den USA entspricht (Vesterby 2003, S. 4; USDA 2003c). ${ }^{97}$

Mit der Farm Bill im Jahr 1996 wurde erneut eine weitreichende Anpassung der ökologischen Ziele des CRP vorgenommen, in der neben den ursprünglich alleinig berücksichtigten erosionsgefährdeten Flächen die Kategorie der umweltsensiblen Flächen eingeführt wurde. Innerhalb des Programms wird nun zwischen umweltsensiblen und erosionsgefährdeten Flächen differenziert (Osborn 1997, S. 291).

Für umweltsensible Flächen, wie beispielsweise Ackerrandstreifen oder Windschutzhecken, sieht das CRP keine Bepflanzungsvorschriften vor. Da sie aus sich selbst heraus eine hohe Bedeutung für den Umweltschutz besitzen, sind sie in ihrem Zustand zu belassen. Erosionsgefährdete Flächen hingegen müssen je nach Region beispielsweise mit Gräsern, Bäumen oder anderweitig bepflanzt und so vor einer Erosion geschützt werden (Osborn 1997, S. 291; Plankl 1999, S. 167). ${ }^{98}$

Methodische Anpassungen haben sich vor allem hinsichtlich der Bewertung der Ackerflächen vollzogen. So wurde von der Einführung des CRP bis zum Jahr 1990 lediglich anhand der Erodierbarkeit der Flächen entschieden. Im Zuge der

\footnotetext{
97 Die gesamte Ackerfläche der USA betrug 1997 rund 455 Millionen acres oder 184 Millionen Hektar (Vesterby 2003, S. 4)

98 Die umweltsensiblen und die erosionsgefährdeten Flächen bilden dabei eine Art Vernetzungsstruktur (Plankl 1999, S. 167).
} 
Ausweitung der Ziele auch auf Aspekte des Schutzes von Gewässern und biotischer Umwelt wurde es jedoch notwendig, auch den Bewertungsmaßstab anzupassen und auszuweiten (Osborn et al. 1995, S. 1-6; Smith 2003, S. 6). Daher wurde 1991 ein Umweltindikator zur Bewertung der angebotenen Flächen eingeführt. Dieser so genannte Environmental Benefits Index (EBI) ermöglicht eine spezifische Bewertung der angebotenen Flächen und die Bildung einer Rangfolge der Angebote, auf deren Grundlage eine Entscheidung über die in das Programm einzubeziehenden Flächen getroffen werden kann (Feather et al. 1998, S. 21; Mello et al. 2002, S. 86). Der EBI selbst wurde seit seiner Einführung ebenfalls periodisch angepasst, worauf in Abschnitt 5.3.3 eingegangen wird.

Im Zuge der Ausweitung der Flächen auf die Kategorien umweltsensible und erosionsgefährdete Flächen erfolgte ebenfalls eine Erweiterung des Ausschreibungsverfahrens. Neben der zeitlich begrenzten Ausschreibung erosionsgefährdeter Ackerflächen wurde für die umweltsensiblen Flächen ein ausschließlich an der Höhe der geforderten Ausgleichszahlung orientiertes Ausschreibungsverfahren $^{99}$ eingeführt. Dabei können die Landwirte ohne Begrenzung durch einen bestimmten Ausschreibungszeitraum jederzeit ein Angebot abgeben (Osborn 1997, S. 291).

Zum Zeitpunkt der Vollendung dieser Arbeit stellt die zwischen Mai und Juni 2003 durchgeführte und insgesamt 26. Ausschreibungsrunde den für die weiteren Betrachtungen aktuellsten Stand des Conservation Reserve Program dar (USDA 2003c). ${ }^{100}$

\subsubsection{Ausschreibungsdesign}

Das CRP ist ein freiwilliges Programm, an dem für Landwirte grundsätzlich die Möglichkeit besteht, mit einzelnen oder allen von ihnen bewirtschafteten Flächen teilzunehmen. Nachfolgend werden mit der Abgrenzung der teilnahmeberechtigten Landwirte und Flächen, der Zuschlagserteilung, der Vertragslaufzeit und einer

\footnotetext{
${ }^{99}$ Das so genannte „,continuous signup“.

${ }^{100}$ Neben dem CRP hat das USDA weitere, in ihrem Ausmaß jedoch weit weniger umfangreiche Konservierungsprogramme implementiert. Beispielsweise das ergänzende Wetlands Reserve Program (WRP) oder das Environmental Quality Incentives Program (EQIP), welches ein in seiner Methodik dem CRP vergleichbares Programm darstellt (Claasen et al. 2001).
} 
zusammenfassenden Einordnung die wesentlichen Merkmale des Ausschreibungsdesigns des CRP betrachtet.

\section{Teilnahmeberechtigte Landwirte und Flächen}

An die Gruppe der potentiell als Teilnehmer in Frage kommenden Landwirte bestehen insofern formale Anforderungen, als dass sie für mindestens 12 Monate vor der entsprechenden Ausschreibungsrunde Eigentümer, Bewirtschafter oder Pächter der angebotenen Fläche waren und zudem glaubhaft machen können, dass sie dies die gesamte Vertragslaufzeit hindurch bleiben (USDA 2003a, USDA 2003b).

Ebenfalls existieren hinsichtlich der anzubietenden Flächen weitreichende Vorgaben, die sich seit der Einführung des CRP schrittweise verändert und an neue Zielausrichtungen angepasst haben. Grundsätzlich sind nicht alle von landwirtschaftlichen Betrieben bewirtschaftete Flächen teilnahmeberechtigt, sondern ausschließlich jene Flächen, die eindeutig definierte Anforderungen erfüllen. Zu Beginn des CRP waren dies Ackerflächen, die als besonders erosionsgefährdet eingestuft wurden. Die Einstufung erfolgte seit 1987 durch den Erodibility Index (EI) und Flächen mit einem EI größer als acht galten als teilnahmeberechtigt. Die Rangordnung erosionsgefährdeter Flächen durch den EI wurde beibehalten und stellt bis heute eine maßgebliche Klassifizierung dar. Jedoch wurden im Zuge umfassender Zielanpassungen weitere Definitionen teilnahmeberechtigter Flächen hinzugenommen, welche sich durch ihre Lage in einem als prioritär eingestuften Gebiet auszeichnen (Osborn et al. 1995, S. 3-4). Tabelle 5-1 gibt eine Übersicht der teilnahmeberechtigten Flächen und ihrer kategoriellen Abgrenzungen.

Tab. 5-1: Übersicht über die 1997 teilnahmeberechtigten Flächen im CRP

\begin{tabular}{|l|c|}
\hline Kategorie (Boden- und Standortbedingungen) & Million acres \\
\hline Besonders erosionsgefährdetes Ackerland (EI > 8) & 142 \\
\hline Ackerland in nationalen prioritären Gebieten & 86 \\
\hline Ackerland in bundesstaatlichen prioritären Gebieten & 24 \\
\hline An Gewässer angrenzendes Ackerland & 13 \\
\hline Bestellte Feuchtgebiete und angrenzendes Hochland & 8 \\
\hline An Gewässer angrenzendes Grünland & Nicht verfügbar \\
\hline Summe der im CRP teilnahmeberechtigten Flächen & 240 \\
\hline
\end{tabular}

Quelle: Eigene Darstellung nach Osborn 1997, S. 291.

${ }^{101}$ Doppelzählungen von Flächen wurden abgezogen. 
Die Darstellung zeigt, dass 1997 über die Hälfte der 455 Millionen acres Ackerland in den USA am CRP teilnahmeberechtigt waren, was rund $18 \%$ der gesamten landwirtschaftlichen Nutzfläche der USA entspricht und die Dimension des Programms zeigt. ${ }^{102}$

\section{Angebotsabgabe und Zuschlagserteilung}

Hinsichtlich der Ausgestaltung der Zuschlagserteilung erfolgt innerhalb des CRP die bereits angesprochene Unterscheidung zwischen erosionsgefährdeten und umweltsensiblen Flächen.

Für die erosionsgefährdeten Flächen können alle Landwirte mit ihren berechtigten Flächen an dem Ausschreibungsverfahren teilnehmen und ein individuelles Angebot abgeben. Vor der Angebotsabgabe ist es für den Landwirt notwendig, sich bei dem für ihn zuständigen regionalen Büro des National Resource Conservation Service (NRCS) über die aktuell zu gewährende Zahlungsrate zu informieren, welche sich an der regionalen Ertragsfähigkeit der Böden orientiert und die maximal mögliche Ausgleichszahlung für eine Fläche darstellt. Unter Berücksichtigung dieser Ausgleichszahlung ermittelt der Landwirt ein Angebot, für welchen Preis er bereit ist eine eindeutig beschriebene Leistung auf einer erosionsgefährdeten Fläche zu erbringen.

Alle unter der maximalen Zahlungsrate liegende Angebote werden gesammelt und die jeweiligen Flächen nach Abschluss der Angebotsfrist durch den Environmental Benefits Index aus ökologischer und ökonomischer Perspektive bewertet und in eine Rangfolge gebracht. Die Flächen, deren ermittelter EBI am größten vom durchschnittlichen EBI abweicht, erhalten zuerst einen Zuschlag (Plankl 1999, S. 167-168). Aufgrund seiner Komplexität und großen Bedeutung innerhalb des CRP wird der Environmental Benefits Index im folgenden Abschnitt ausführlich betrachtet.

Für den Sonderfall der umweltsensiblen Flächen erfolgt die Zuschlagserteilung ausschließlich orientiert an der jeweils geforderten Ausgleichzahlung. Für die Festsetzung der maximalen Höhe der Ausgleichszahlung wird ein den

\footnotetext{
${ }^{102}$ Da es durch die Farm Bill 2002 diesbezüglich keine nennenswerten Änderungen gegeben hat, können die Daten zu den teilnahmeberechtigten Flächen auch aktuell als weitestgehend zutreffend angesehen werden (USDA 2002a; 2002b).
} 
erosionsgefährdeten Flächen ähnliches Vorgehen verwendet. Die Grundlage stellt ebenfalls der regional festgelegte maximale Ausgleichszahlungsbetrag dar, welcher um eine Anreizkomponente erhöht werden kann. Der zuschlagsfähige Normalsatz beträgt $20 \%$, wobei je nach Qualität und Lage der Flächen gegebenenfalls weitere Zuschläge gewährt werden können (Plankl 1999, S. 170).

\section{Vertragslaufzeit}

Für die angenommenen Flächen werden zwischen den Landwirten und der Farm Service Agency (FSA) im Namen der Commodity Credit Corporation (CCC) Verträge geschlossen, die alle für die Vertragslaufzeit relevanten Aspekte beinhalten. Die Vertragslaufzeit innerhalb des CRP beträgt für stark erosionsgefährdete Ackerflächen in der Regel 10 Jahre und eine Ausdehnung auf einen Zeitraum von bis zu 15 Jahren ist nur für als besonders wertvoll eingestufte Umweltleistungen möglich. Die Vertragslaufzeit für eine Fläche darf 15 Jahre nicht überschreiten (USDA 2003b).

Für beide Flächenkategorien gilt, dass Flächen deren Vertragslaufzeit beendet ist, ebenso wie Flächen die in einer vorherigen Ausschreibungsrunde nicht angenommen wurden, erneut angeboten werden können. Bis 2002 war es vorgeschrieben, dass Flächen deren Vertrag ausläuft mit allen weiteren in der Ausschreibungsrunde angebotenen Flächen konkurrieren müssen. Durch die Farm Bill 2002 wurde dies geändert und Flächen die Teil des CRP geworden sind, qualifizieren sich nach Vertragsablauf automatisch für eine erneute Berücksichtigung (USDA 2002a; 2002b). Entschließt sich ein Landwirt nach Ablauf der Vertragslaufzeit gegen ein erneutes Angebot, können die Flächen ohne Bewirtschaftungsauflagen wieder als Ackerflächen genutzt werden.

Der obigen Klassifizierung entsprechend kann die dem CRP zugrunde liegende Ausschreibung zusammenfassend als preisdiskriminierende Mehrgüterausschreibung mit regionalisierten Ausschlussgrenzen und der Zuschlagserteilung unter Berücksichtigung ökonomischer und ökologischer Kriterien in Form des EBI charakterisiert werden. 


\subsubsection{Environmental Benefits Index}

Innerhalb des Ausschreibungsverfahrens stellt der Umweltindikator Environmental Benefits Index (EBI) das maßgebliche Instrument dar. Ihm kommt in diesem Zusammenhang eine Doppelfunktion zu, denn durch den EBI werden die angebotenen Flächen aus ökologischer und ökonomischer Sicht bewertet und darauf aufbauend eine Rangfolge dieser Flächen erstellt.

Seit seiner Einführung im Jahr 1991 unterlag der EBI einem kontinuierlichen Prozess, in dem periodische Anpassungen und Verbesserungen seiner Faktoren und ihrer Gewichtungen vorgenommen wurden (Feather et al. 1998, S. 21). Anfangs wurde der EBI auf der Grundlage der folgenden sieben gleichgewichteten Faktoren berechnet: Oberflächenwasserqualität, Grundwasserqualität, Ertragspotential des Bodens, Beitrag zum Erosionsschutz, Neuanpflanzungen von Bäumen, Nähe zu ausgewiesenen Wasserschutzgebieten und Nähe zu Vorrangflächen (USGAO 1993, S. 12-13).

Die exakte Zusammensetzung des EBI war den Landwirten anfangs jedoch noch unbekannt (Szentandrasi et al. 1995, S. 387). Nach drei Ausschreibungsrunden wurde die Struktur des EBI 1995 öffentlich gemacht und dahingehend weiterentwickelt, dass er sich fortan aus vier unterschiedlich gewichteten ökologischen Faktoren und einem neu eingeführten Kostenfaktor zusammensetzte. Innerhalb der ökologischen Faktoren Wasserqualität, Artenschutz und Erosionsschutz konnten jeweils maximal 20 Punkte, bei Neuanpflanzungen von Bäumen maximal 10 Punkte erreicht werden. Der Kostenfaktor hing von der geforderten jährlichen Ausgleichszahlung ab (Osborn 1997, S. 290; Ribaudo et al. 2001, S. 15).

1997 erfolgte die nächste grundlegende Veränderung des EBI. Der Index bestand seit dem aus sechs Umweltfaktoren und einem Kostenfaktor. Für die Umweltfaktoren Artenschutz, Wasserqualität und Erosionsschutz konnten bis zu 100 Punkte, für den Nachhaltigkeitsfaktor bis zu 50 Punkte und für die Faktoren Luftqualität und Vorrangflächen bis zu 25 Punkte erreicht werden. Der Kostenfaktor wurde mit maximal 150 Punkten bewertet (Osborn 1997, S. 292; Smith 2003, S. 4).

Im Anschluss wurde der EBI nur noch geringfügig verändert und im Zuge der letzten Ausschreibungsrunde wurde seitens des USDA der Faktor Vorrangflächen entfernt, 
so dass sich der EBI aktuell aus den folgenden sechs Faktoren zusammensetzt (Mello et al. 2002, S. 87-88; Smith 2003, S. 4; USDA 2003d).

\section{Artenschutz}

Der Artenschutzfaktor bewertet den Nutzenbeitrag der angebotenen Flächen für den Schutz standortgemäßer Pflanzen- und Tierarten und vom Aussterben bedrohter Arten, so dass hier zusammenfassend Art, Umfang und Lage der Pflanzendecken berücksichtigt werden. Insgesamt können mit dem Artenschutzfaktor 100 Punkte erreicht werden, wobei sich der Faktor aus drei Unterfaktoren zusammensetzt. Für den Unterfaktor Vegetationsdecke können maximal 50 Punkte, für den Unterfaktor Eignung als Nistplatz bis zu 20 Punkte und für die Nähe zu einem geschützten Lebensraum höchstens 30 Punkte erreicht werden.

\section{Wasserqualität}

Bewertet wird hier der Nutzen für die Wasserqualität unter Berücksichtigung ausgewiesener Qualitätswasserflächen, der von einer hohen Trinkwasserqualität profitierenden Bevölkerung und einer Lage der Fläche in einem ausgewiesenen Schutzgebiet. Innerhalb des Wasserqualitätsfaktors können 100 Punkte erreicht werden, welche sich aus maximal 30 Punkten für die Nähe zu Wasserschutzgebieten, höchstens 25 Punkten für die Grund- und bis zu 45 Punkten für die Oberflächenwasserqualität zusammensetzen.

\section{Erosionsschutz}

Der Erosionsschutzfaktor zielt auf das Potential zur Reduzierung der Wasser- und Winderosion durch die angebotenen Flächen ab. Der Faktor ist hinsichtlich der Flächenbewertung identisch mit dem 1987 eingeführten Erodibility Index und ermöglicht eine zu erreichende Punktzahl von bis zu 100 .

\section{Nachhaltigkeit}

Mit dem Nachhaltigkeitsfaktor wird die Wahrscheinlichkeit bewertet, mit der die vertraglich vereinbarten Praktiken auch über das Ende der Vertragslaufzeit hinaus beibehalten werden. Neuanpflanzungen von Bäumen wird bei der Bewertung beispielsweise eine höhere Nachhaltigkeit als bestehenden Gehölzen oder Grasanpflanzungen unterstellt. Hier können maximal 50 Punkte erreicht werden. 


\section{Luftqualität}

Dieser Faktor lässt die Bewertung der potentiellen Verbesserung der Luftqualität in die Entscheidung einfließen. Die zu erreichenden Punkte belaufen sich insgesamt auf bis zu 45, wovon 25 auf den Einfluss einer Reduzierung der Winderosion entfallen. Maximal 15 Punkte können durch einen Beitrag zur Reduzierung von Treibhausgasen und bis zu 5 Punkte für den Fall erreicht werden, dass 51 \% oder mehr der Fläche in einer Luftqualitätszone liegen.

\section{Kosten}

Der Kostenfaktor setzt sich aus drei Bestandteilen zusammen, von denen zwei den Landwirten vorab hinsichtlich der erreichbaren Punktzahl bekannt sind. Dies ist zum einen der Verzicht auf einen Kostenzuschuss für eine Erstanpflanzung, was mit maximal 10 Punkten honoriert wird. Zum anderen erhalten Angebote für jeden Dollar, den die geforderte jährliche Zahlung pro acre unterhalb der maximalen Zahlungsrate liegt, einen Punkt. Hier dürfen maximal 15 Punkte vergeben werden.

Der dritte und mit Abstand umfassendste Anteil kommt dem durch den jeweiligen Angebotspreis bestimmten Angebotsfaktor zu. Die für diesen Faktor grundsätzlich zu vergebenden Punkte sind den Landwirten nicht bekannt, da sie erst nach Abschluss der Angebotsfrist festgelegt werden. Demzufolge besteht die Möglichkeit, durch die Wahl der dritten Komponente des Kostenfaktors eine Schwerpunktsetzung in eine ökonomische oder eine ökologische Richtung zu vollziehen, was zur Folge hat, dass das relative Gewicht des gesamten Kostenfaktors innerhalb des EBI erst nach dieser Wahl feststeht. Grundsätzlich ist festzuhalten, dass mit einem steigenden Angebotspreis sowohl die erreichbare Punktzahl und als auch die Wahrscheinlichkeit einer Zuschlagserteilung sinken. Unabhängig von der finalen Ausgestaltung stellt der Kostenfaktor den Faktor mit den meisten Punkten innerhalb des EBI dar. 
Einen Überblick über die Zusammensetzung des EBI in der 26. Ausschreibungsrunde gibt Tabelle 5-2.

Tab. 5-2: Zusammensetzung des EBI in der 26. Ausschreibungsrunde

\begin{tabular}{|l|c|}
\hline EBI-Faktor & Maximal erreichbare Gesamtpunktzahl \\
\hline Artenschutz & 100 \\
\hline Wasserqualität & 100 \\
\hline Erosionsschutz & 100 \\
\hline Nachhaltigkeit & 50 \\
\hline Luftqualität & 45 \\
\hline Kostenfaktor & $25+$ Angebotsfaktor \\
\hline
\end{tabular}

Quelle: Eigene Darstellung nach Mello et al. 2002, S. 88 und aktualisiert unter Bezug auf USDA 2003c und Smith 2003, S. 4.

Die Verwaltungseinbindung ist so ausgestaltet, dass die erosionsgefährdeten Flächen hinsichtlich der fünf Umweltfaktoren durch die jeweiligen regionalen Büros des National Resource Conservation Service (NRCS) auf der Grundlage von regionsspezifisch erhobenen Daten beurteilt werden. Getrennt davon wird der Kostenfaktor seitens der FSA nach einer festgelegten Gewichtungsformel bewertet und die entsprechenden Punktzahlen für die Angebote vergeben (Mello et al. 2002, S. 87).

\subsubsection{Bewertung}

Zusammenfassend hat sich gezeigt, dass mit dem CRP durch die Kombination eines Ausschreibungsverfahrens mit einem Umweltindikator ein nunmehr langjährig eingesetztes und praktikables Verfahren zur gemeinsamen Berücksichtigung ökologischer und ökonomischer Aspekte bei der Auswahl zu honorierender Flächen Einzug in die Agrarumweltpolitik der USA gefunden hat.

Im Laufe der praktischen Umsetzung ist es zudem gelungen, sukzessive methodische Anpassungen und Weiterentwicklungen vorzunehmen und die aus ökonomischer Sicht maßgebliche Effizienz durch eine Reduzierung der flächenbezogenen Ausgleichszahlungen zu erreichen (Plankl 1999, S. 171-173; Mello et al. 2002, S. 9091).

Die große Bedeutung des CRP belegen zudem einige Zahlen zur Inanspruchnahme des Programms, wobei hier die Ergebnisse der 26. Ausschreibungsrunde betrachtet werden (USDA 2003c). Im Rahmen dieser Ausschreibung sind landesweit insgesamt 
71.077 Angebote eingegangen, die rund 4,1 Millionen acres entsprechen. Davon konnten 38.621 Angebote mit rund 2 Millionen acres Ackerfläche angenommen werden und der erreichte EBI dieser Flächen beträgt landesweit ohne den Kostenfaktor 210 Punkte und mit dem Kostenfaktor 302 Punkte. Der durchschnittliche Auszahlungsbetrag liegt landesweit bei \$ 56,53 pro acre und reicht von \$ 20,98 pro acre in Kalifornien bis \$ 101,01 pro acre in Iowa (USDA 2003c). Tabelle 5-3 gibt eine Zusammenfassung der landesweiten Ergebnisse der im Jahr 2003 durchgeführten 26. Ausschreibungsrunde im CRP. ${ }^{103}$

Tab. 5-3: Ergebnisse der 26. Ausschreibungsrunde im CRP

\begin{tabular}{|l|c|}
\hline & Gesamte USA \\
\hline Eingegangene Angebote & $71.077(4.148 .989$ acres $)$ \\
\hline Angenommene Angebote & 38.621 (1.995.189 acres) \\
\hline Durchschnittlicher EBI (ohne Kosten) & 210 \\
\hline Durchschnittlicher EBI (mit Kosten) & 302 \\
\hline Durchschnittlicher Auszahlungsbetrag & \$56,53 pro acre \\
\hline
\end{tabular}

Quelle: Eigene Zusammenstellung nach Daten aus USDA 2003c.

Insgesamt waren zum Zeitpunkt Oktober 2003 über 34 Millionen acres vertraglich in das CRP einbezogen, was rund 7,6 \% der gesamten Ackerfläche der USA entspricht und sowohl die Akzeptanz als auch den Stellenwert des CRP verdeutlicht (USDA 2003c).

Im Hinblick auf die hier verfolge Betrachtung von Transaktionskosten eines Ausschreibungsverfahrens ist abschließend anzumerken, dass bislang keine Untersuchung gefunden werden konnte, welche sich mit der Ermittlung und Bewertung der Transaktionskosten der am CRP teilnehmenden Landwirte auseinandergesetzt hat.

\subsection{Weitere Konzepte des Einsatzes von Ausschreibungen}

Im Anschluss an das CRP wird der Blick auf zwei weitere Ansätze gelenkt, in denen der praktische Einsatz eines Ausschreibungsverfahrens für Umweltleistungen erprobt wurde. Dabei werden das Programm „BushTender Trial“ aus Australien und ein

\footnotetext{
${ }^{103}$ Eine detaillierte Übersicht der Ergebnisse für alle Bundesstaaten findet sich in USDA 2003c.
} 
Modellausschreibungen beinhaltendes Programm der Universität Bonn in NordrheinWestfalen jeweils kurz betrachtet. ${ }^{104}$

\subsubsection{BushTender Trial in Australien}

Die Erprobung von Ausschreibungen hat in Australien erstmals im Rahmen des so genannten BushTender Trial in Victoria stattgefunden (Stoneham et al. 2003, S. 477478; Figgis 2003, S. 214; OECD 2004, S. 69). Der sich grundlegend von bestehenden Programmen in Australien unterscheidende Ansatz wurde 2001 in zwei Projektregionen ${ }^{105}$ eingeführt, um einen neuen Weg zum Schutz heimischer Biodiversität durch eine entsprechende Bewirtschaftung in privatem Besitz befindlicher Flächen zu evaluieren (NECMA 2005).

Teilnahmeberechtigt waren alle Landbesitzer innerhalb der Modellregionen mit ihren entsprechenden Flächen. Sie waren aufgerufen Angebote abzugeben, zu denen sie bereit sind Umweltleistungen zum Schutz und zum Erhalt von Biodiversität durchzuführen und diese für eine Vertragslaufzeit von drei Jahren beizubehalten (NECMA 2005). Im Vorfeld der Angebotsabgabe wurden die teilnahmeberechtigten Flächen interessierter Landbesitzer durch Ökologen begutachtet und ein Managementplan erstellt (Stoneham et al. 2003, S. 490; NECMA 2005).

Die eingegangenen Angebote wurden mit einem Umweltindex auf der Basis der bestehenden Umweltqualität der Fläche, der zusätzlich angebotenen Leistungen und der dafür geforderten Zahlung in eine Rangfolge gebracht. Der Umweltindikator ermöglichte somit, die Angebote sowohl nach ökologischen als auch nach

\footnotetext{
104 Über die in dieser Arbeit angesprochenen Ansätze hinaus haben Ausschreibungen bislang in Australien sowohl im „ALR: Auction for Landscape Recovery“ als auch dem „EcoTender“ und in Großbritannien innerhalb der „Challenge Funds“ ebenfalls einen praktischen Einsatz gefunden. Für einen umfassenden und aktuellen Überblick sei an dieser Stelle auf die Arbeit von Latacz-Lohmann und Schilizzi (Latacz-Lohman und Schilizzi 2005) verwiesen. Neben den genannten Ansätze sind zudem das „Conservation Stewardship Scheme“ und das „Nitrate Sensitive Areas Scheme“ aus Großbritanien anzuführen (Cason und Gangadharan 2004, S. 1211). Sie werden im Rahmen dieser Arbeit jedoch nicht weiter einbezogen, da ihnen eine von den hier und im Forschungsprojekt betrachteten Ausschreibungen grundlegend verschiedene Ausgestaltung zugrunde liegt. Die beiden Programme sind dadurch gekennzeichnet, dass die Landwirte kein individuelles Angebot abgeben, zu welchem Preis sie bereit sind eine definierte Umweltleistung zu erbringen, sondern umgekehrt alle Landwirte eine einheitliche Prämienzahlung erhalten und sie in Form eines individuellen Bewirtschaftungsplans eine Umweltleistung anbieten, die sie im Gegenzug zu erbringen bereit sind. ${ }^{105}$ Die Projektregionen waren die North-Central-Area bei Bendigo und Ballarat und die North-EastArea bei Benalla und Albury in Victoria (Stoneham et al. 2003, S. 478).
} 
ökonomischen Gesichtspunkten zu bewerten und bei der Zuschlagserteilung den ökologischen Nutzen pro eingesetztem Dollar zu maximieren (Latacz-Lohmann 2004, S. 6).

Dabei stand für beide Regionen ein begrenztes Budget von insgesamt \$A 400.000 zur Verfügung und die Angebote wurden bis zur Ausschöpfung dieses Budgets entsprechend der durch den Umweltindex ermittelten Rangfolge angenommen und nach dem Vertragsabschluss erfolgten die Zahlungen an die Landbesitzer jeweils jährlich im Anschluss an die Überprüfung der vertragskonformen Leistungserbringung.

Insgesamt sind in der ersten Ausschreibungsrunde für beide Regionen zusammen 126 Interessenbekundungen eingegangen, denen nach der Flächenbegutachtung 98 Angebote für 186 Flächen folgten. Angenommen wurden letztlich 73 Angebote über 131 Flächen, die einer vertraglich berücksichtigten Gesamtfläche von 3.160 Hektar entsprechen (Stoneham et al. 2003, S. 490-491). Die Ergebnisse der ersten Ausschreibungsrunde im Rahmen des BushTender Trial sind in Tabelle 5-4 für beide Regionen zusammen dargestellt.

Tab. 5-4: Ergebnisse der ersten Ausschreibungsrunde - BushTender Trial

\begin{tabular}{|l|c|c|c|}
\hline & $\begin{array}{c}\text { Interessen- } \\
\text { bekundungen }\end{array}$ & $\begin{array}{c}\text { Eingegangene } \\
\text { Angebote }\end{array}$ & $\begin{array}{c}\text { Angenommene } \\
\text { Angebote }\end{array}$ \\
\hline Teilnehmer & 126 & 98 & 73 \\
\hline Flächenanzahl & 223 & 186 & 131 \\
\hline Flächengröße in ha & 3.846 & 3.478 & 3.160 \\
\hline
\end{tabular}

Quelle: Eigene Darstellung nach Stoneham et al. 2003, S. 491.

Im Hinblick auf die Angebotspreise ist anzuführen, dass bedingt durch die Zuschlagserteilung anhand des Umweltindikators in der Literatur keine über den Wert von rund \$A 4.600 als Mittelwert der Angebotshöhe pro Betrieb hinausgehenden Angaben ermittelt werden konnten (Stoneham et al. 2003, S. 493).

In einer zweiten Ausschreibungsrunde im Jahr 2002 wurde das erfolgreiche Konzept beibehalten und auf weitere Regionen ausgeweitet (Macken 2002, S. 81). ${ }^{106}$ Darüber hinaus hat in der Folge des BushTender Trial das Interesse an Ausschreibungen in Australien stark zugenommen und als Teil des nationalen „Market-based Instruments

\footnotetext{
${ }^{106}$ Diesbezüglich sind derzeit noch keine Ergebnisse verfügbar.
} 
Pilots Program“ (MBI) sind weitere Programme unter Einbeziehung von Ausschreibungsverfahren sowohl geplant als auch bereits in der Umsetzung befindlich (NAP 2002; NAP 2005).

Hinsichtlich einer Ermittlung und Bewertung von Transaktionskosten der Landwirte im Rahmen der Durchführung des BushTender Trial können bislang keine Arbeiten berücksichtigt werden.

\subsubsection{Modellausschreibungen in Nordrhein-Westfalen}

Zur Erprobungen der Möglichkeiten einer Implementierung von Ausschreibungen in das Kulturlandschaftsprogramm von Nordrhein-Westfalen wurden vom Ministerium für Umwelt und Naturschutz, Landwirtschaft und Verbraucherschutz NordrheinWestfalen Modellausschreibungen in Auftrag gegeben und von der Universität Bonn durchgeführt (Holm-Müller und Hilden 2005). ${ }^{107}$

Das grundlegende Ziel der Untersuchungen war die Beantwortung der Frage, ob der Einsatz von Ausschreibungen dazu beitragen kann, die Teilnehmerzahl an Agrarumweltprogrammen ökonomisch vertretbar zu erhöhen. Dazu wurden der Landkreis Minden-Lübbecke und der Landkreis Wesel ausgewählt, in denen exemplarisch überprüft wurde, ob eine Grünlandextensivierung für Landwirte auf ertragreichen Standorten attraktiver gemacht werden kann. Beide Landkreise waren durch eine mittlere bis hohe Intensität der Viehhaltung und eine zu geringe Teilnahme im bisherigen Grünlandextensivierungsprogramm des Landes NordrheinWestfalen gekennzeichnet. Das erste Ausschreibungsverfahren in den Modellregionen hat im Jahr 2003, das zweite im Jahr 2004 stattgefunden.

Dem Modellvorhaben lag kein reines Ausschreibungsverfahren, sondern eine Kombination aus einer einheitlichen Prämienzahlung und einer Ausschreibungskomponente zugrunde. Die teilnehmenden Landwirte haben ergänzend zu dem regulären Antrag für die MSL-Grünlandextensivierung einen Zusatzantrag im Rahmen des Ausschreibungsverfahrens gestellt. Darin wurde ein

\footnotetext{
${ }^{107}$ Die weiteren Ausführungen zu den Modellausschreibungen in Nordrhein-Westfalen beziehen sich auf Holm-Müller und Hilden (2005).
} 
Angebot abgegeben, zu welchem Gesamtpreis (Zusatzantrag + Prämienzahlung) der Landwirt bereit war, eine Grünlandextensivierung durchzuführen.

Als Zuschlagskriterium fungierte weder die Gesamtprämie, noch die Zusatzprämie, sondern das Verhältnis der geforderten Zusatzprämie aus dem Ausschreibungsverfahren zur Prämie aus dem MSL-Antrag, wodurch die Extensivierungsmaßnahmen mit unterschiedlichen Prämienhöhen aus dem MSLProgramm Berücksichtigung finden konnten. Alle Landwirte, die unterhalb der festgelegten Ausschlussgrenze lagen, wurden zu den jeweils von ihnen angebotenen Konditionen in das Programm aufgenommen. ${ }^{108}$

An beiden Ausschreibungsrunden haben jeweils 15 landwirtschaftliche Betriebe teilgenommen, wobei im Jahr 2003 insgesamt 335 Hektar und im Jahr 2004 insgesamt 353 Hektar angeboten wurden. Die geforderten Zusatzprämien umfassen im ersten Ausschreibungsverfahren eine Spanne von $60 €$ pro Hektar bis $150 €$ pro Hektar und im zweiten Ausschreibungsverfahren einen Bereich von $20 €$ pro Hektar bis $195 €$ pro Hektar. Die durchschnittlich geforderte Zusatzprämie je Hektar beträgt $92 €$ im Jahr 2003 und $46 €$ im Jahr 2004.

Die Ausschlussgrenze wurde im ersten Jahr auf 53 \% und im zweiten Jahr auf 43 \% festgelegt. Somit haben die Landwirte einen Zuschlag erhalten, deren zusätzliche Prämienforderung aus dem Ausschreibungsverfahren nicht mehr als 53 \% bzw. 43 \% der MSL-Prämie betragen hat. Im ersten Ausschreibungsverfahren haben neun Betriebe mit einer Gesamtfläche von 218 Hektar einen Zuschlag erhalten. Die Zusatzprämien dieser Betriebe reichen von $47 €$ je Hektar bis $80 €$ je Hektar und weisen eine durchschnittliche Forderung von $73 €$ pro Hektar auf. Innerhalb des zweiten Ausschreibungsverfahrens haben 14 Betriebe mit einer Fläche von 342 Hektar einen Zuschlag erhalten und die Zusatzprämien der angenommenen Betriebe umfassen mit einer durchschnittlichen Forderung je Hektar in Höhe von $46 €$ eine Spanne von $20 €$ pro Hektar bis $195 €$ pro Hektar.

\footnotetext{
108 Zum Zeitpunkt der Erstellung dieser Arbeit kann ausschließlich auf die Ergebnisse der ersten beiden Ausschreibungsverfahren zurückgegriffen werden.
} 
Die Ergebnisse der angenommenen Angebote sind für die erste und die zweite Ausschreibungsrunde in Tabelle 5-5 dargestellt.

Tab. 5-5: Angenommene Angebote der ersten und zweiten Modellausschreibung in Nordrhein-Westfalen

\begin{tabular}{|l|c|c|}
\hline & $\begin{array}{c}\text { Ausschreibung } \\
2003\end{array}$ & $\begin{array}{c}\text { Ausschreibung } \\
2004\end{array}$ \\
\hline Betriebe & 9 & 14 \\
\hline Zusatzprämie in $€$ / ha (Mittelwert) & 73 & 46 \\
\hline Ausschlussgrenze in \% & 53 & 43 \\
\hline Gesamtfläche in ha & 218 & 342 \\
\hline
\end{tabular}

Quelle: Eigene Darstellung nach Holm-Müller und Hilden 2005.

Zusammenfassend hat sich auch hier gezeigt, dass Ausschreibungskomponenten zur Honorierung von Umweltleistungen sowohl praktisch umsetzbar sind als auch seitens der Landwirte akzeptiert werden und zu einer Aufdeckung und Berücksichtigung differenzierter Angebotspreise geführt haben.

Hinsichtlich der mit den Ausschreibungsverfahren verbundenen Transaktionskosten geben Holm-Müller und Hilden auf der Grundlage einer Befragung von Landwirten und Beratern die Einschätzung ab, dass durch die Ausschreibungen im Vergleich zu Prämienzahlungen keine oder nur geringe zusätzliche Transaktionskosten entstanden sind. $^{109}$

109 Es ist anhand der vorliegenden Literatur jedoch nicht ersichtlich, welche Kosten dabei im Einzelnen als Transaktionskosten definiert wurden. 


\section{Die transaktionskostenökonomische Analyse}

Bevor in den anschließenden Kapiteln die empirischen Untersuchungen erörtert werden, thematisieren die nachfolgenden Abschnitte die Anwendung des transaktionskostenökonomischen Analyserahmens auf den Untersuchungsgegenstand der Transaktionsbeziehung im Rahmen von Ausschreibungen zur Honorierung ökologischer Leistungen der Landwirtschaft.

Hierbei ist ein besonderer Stellenwert auf den wesentlichen Unterschied einer Ausschreibung gegenüber einer Auktion zu legen, welcher die Notwendigkeit der über den Zeitpunkt des Vertragsabschlusses (der Zuschlagserteilung) hinausgehenden Betrachtung verdeutlicht. Die innerhalb der Auktionstheorie maßgeblich thematisierte Verkaufsauktion ist in der Regel nach der Zuschlagserteilung erfüllt. Demgegenüber umfasst die Transaktionsbeziehung im Rahmen eines Ausschreibungsverfahrens für Umweltleistungen einen über den Vertragsabschluss hinausgehenden Zeitraum, da die Kontrolle der Leistungserbringung und die entsprechende Entlohnung zu einem mitunter wesentlich späteren Zeitpunkt erfolgen. ${ }^{110}$

Folglich gilt es sowohl den Zeitraum bis zur Zuschlagserteilung als auch darüber hinaus bis zur Leistungserbringung hinsichtlich der Ausprägung der Verhaltensannahmen der Akteure und der Dimensionen der Transaktionsbeziehung zu betrachten. Die nachfolgende Argumentation impliziert neben dieser Anwendung des transaktionskostenökonomischen Analyserahmens zudem jeweils eine spezifische Würdigung der einzelnen Bestandteile vor dem Hintergrund ihrer praktischen Relevanz und Aussagekraft.

Hierzu werden in Abschnitt 6.1 einleitend die grundlegenden Aspekte der zu untersuchenden Vertragsbeziehung dargelegt und die Akteure Agrarverwaltung und Landwirte charakterisiert.

Im Anschluss erfolgen in Abschnitt 6.2 die Einbeziehung der das Menschenbild innerhalb der Transaktionskostenökonomik kennzeichnenden Verhaltensannahmen und die Analyse ihrer jeweiligen Ausprägungen aus Sicht der Agrarverwaltung und der Landwirte. Dem Ansatz der Transaktionskostenökonomik folgend, thematisiert

\footnotetext{
${ }^{110}$ In Agrarumweltprogrammen in der Regel jährlich.
} 
Abschnitt 6.3 die Eigenschaften der Transaktionsbeziehung anhand ihrer Dimensionen und unter Einbeziehung des Konzepts der fundamentalen Transformation. Aufbauend auf den gewonnenen Erkenntnissen wird in Abschnitt 6.4 die Ableitung einer Beherrschungsstruktur der Ausschreibung ökologischer Leistungen diskutiert.

Zum Abschluss des Kapitels erfolgt in Abschnitt 6.5 eine zusammenfassende Betrachtung der transaktionskostenökonomischen Analyse. Im Zuge dessen wird das in dieser Arbeit gewählte Ausschreibungsdesign einbezogen und eine Würdigung der Anwendbarkeit des konzeptionellen Rahmens der Transaktionskostenökonomik auf die hier zugrunde liegende Transaktionsbeziehung vorgenommen.

\subsection{Grundlagen}

Die Transaktionsbeziehung in einem Ausschreibungsverfahren zur Honorierung von Umweltleistungen ist dadurch gekennzeichnet, dass es sich bei den gehandelten Gütern um öffentliche Güter handelt. Ein Markt muss folglich staatlich geschaffen werden und die Agrarverwaltung tritt dabei als Nachfrager, die Landwirte als Anbieter auf. Zur Verdeutlichung erfolgt einführend eine Beschreibung der relevanten Akteure und im Anschluss wird die Transaktionsbeziehung in der Terminologie der Transaktionskostenökonomik erläutert.

\section{Agrarverwaltung}

Sowohl für die gesamte öffentliche Verwaltung als auch für die Agrarverwaltung haben sich in Deutschland keine allgemeingültigen und umfassenden Definitionen etabliert (Schedler 1993, S. 15; Warnemünde 1995, S. 14; Schedler und Proeller 2000, S. 3-23). Somit wird hier ebenfalls eine grobe Abgrenzung vorgenommen und die öffentliche Verwaltung ganz allgemein der Exekutive des Staates zugeordnet, die als vollziehende Gewalt die Erfüllung öffentlicher Aufgaben auf der Grundlage von Gesetzen und Verordnungen verfolgt (Schedler 1993, S. 15-16; Schedler und Proeller 2000, S. 14-15).

Die Agrarverwaltung stellt in diesem Zusammenhang den sachlich abgegrenzten Teilbereich der öffentlichen Verwaltung dar, der dafür verantwortlich ist, dass die Agrarumweltprogramme effizient, wirksam und ordnungsgemäß verwaltet und 
durchgeführt werden (Warnemünde 1995, S. 14-15; Mann 2001, S. 302-303; Hespelt 2005, S. 129; Art. 75, VO (EG) Nr. 1698/2005).

Bei der Analyse des Ausschreibungsverfahrens können die Agrarverwaltung und der Staat infolgedessen begrifflich grundsätzlich synonym verwendet werden. Im Folgenden wird im Hinblick auf die spezifische Betrachtung der Ausschreibung von Umweltleistungen die Bezeichnung Agrarverwaltung für die ausschreibende und durchführende staatliche Institution verwendet. ${ }^{111}$

Zusammenfassend tritt die jeweilige Agrarverwaltung im Zuge der von ihr durchgeführten Ausschreibung in einem spezifischen Agrarumweltprogramm als Monopolist auf und verfolgt die grundsätzlichen Ziele der Kostenminimierung bzw. der Nutzenmaximierung, des effizienten Mitteleinsatzes und der Informationsgewinnung. ${ }^{112}$

\section{Landwirte}

Im Anschluss an die Agrarverwaltung wird mit den Landwirten der zweite Akteur innerhalb des Ausschreibungsverfahrens charakterisiert, wobei keine Kennzeichnung der einzelnen Ausprägungen landwirtschaftlicher Aktivitäten erfolgt. Vielmehr wird an dieser Stelle eine Beschränkung auf die hier maßgeblichen und erwartungsgemäß für die überwiegende Anzahl der Landwirte relevanten Aspekte vorgenommen.

Mit dem Fokus auf dem Erhalt und der Förderung pflanzlicher Biodiversität auf landwirtschaftlich genutzten Grünlandflächen ist vor allem die Abhängigkeit von dem standortspezifischen Produktionsfaktor Boden und damit verbunden ganz allgemein der starke Einfluss der Natur auf die Leistungserbringung zu betonen. Demzufolge stellt die innerhalb dieser Arbeit relevante landwirtschaftliche Produktion unter Freilandbedingungen ein klassisches Beispiel eines unsicheren Produktionsprozesses dar, der sowohl durch Witterungseinflüsse als auch weitere Faktoren wie Erkrankungen der Pflanzen beeinflusst wird (Beckmann 1997, S. 159161).

Grundsätzlich sind Landwirte innerhalb einer Ausschreibung in der Form zu charakterisieren, dass sie als Einzelunternehmer mit anderen Landwirten in einem Wettbewerb um finanzielle Mittel stehen. Bei einem Ausschreibungsverfahren ist die $\mathrm{zu}$ treffende Entscheidung dementsprechend umfangreicher als bei einer

\footnotetext{
${ }^{111}$ Eine Untersuchung der verwaltungsinternen Abläufe und Anreize wird hier nicht vorgenommen.

112 Siehe dazu auch Abschnitt 5.2.1 dieser Arbeit.
} 
Prämienzahlung, da nicht nur eine Entscheidung für oder gegen die Teilnahme an einem Agrarumweltprogramm zu treffen ist, sondern es zudem unternehmerisch eigenständig zu entscheiden gilt, zu welchem Preis die definierte Umweltleistung auf den Flächen angeboten wird (Latacz-Lohmann 1993, S. 352).

Wie oben angedeutet wurde, wird auf die zu untersuchende Transaktion nachfolgend ausschließlich unter dem Gesichtspunkt der durch die jeweiligen Ziele der Akteure geprägte Austauschbeziehung eingegangen und sowohl die landwirtschaftlichen Betriebe als auch die Agrarverwaltung werden nicht hinsichtlich ihrer internen Organisation untersucht. Diese Transaktion umfasst den Zeitraum von der Bekanntmachung des Ausschreibungsverfahrens über die Angebotsabgabe und den Vertragsabschluss bis hin zur Leistungserbringung. Das Zustandekommen von Agrarumweltprogrammen wird in diesem Zusammenhang somit ausdrücklich nicht betrachtet und entsprechende Programme werden als gegeben angesehen.

In der Terminologie der Transaktionskostenökonomik ist die derzeit zur Honorierung von Umweltleistungen verwendete Einheitsprämie in einem spezifischen Agrarumweltprogramm als ein Standardvertrag anzusehen, da die Vertragsinhalte sowohl hinsichtlich der zu erbringenden Umweltleistung als auch der Entlohnung für alle teilnehmenden Landwirte identisch sind. Demgegenüber entspricht die innerhalb eines Ausschreibungsverfahrens relevante Vertragsausgestaltung einem NichtStandardvertrag. Die ökologische Leistung ist für die jeweiligen Qualitätsstufen identisch, die Honorierung erfolgt jedoch gemäß der individuellen Angebotspreise pro Hektar und folglich wird mit einem Landwirt faktisch für jede Fläche ein individueller Vertrag in Form einer umfangreichen Vertragsdifferenzierung geschlossen.

\subsection{Verhaltensannahmen}

Die innerhalb der Transaktionskostenökonomik zugrunde gelegten Verhaltensannahmen Opportunismus und begrenzte Rationalität kennzeichnen das den handelnden Individuen innewohnende Menschenbild. Im weiteren Verlauf des Kapitels werden diese Verhaltensannahmen jeweils aus Sicht der Agrarverwaltung 
und der Landwirte hinterfragt und hinsichtlich ihrer Ausprägung innerhalb eines Ausschreibungsverfahrens diskutiert.

\subsubsection{Begrenzte Rationalität}

Die Verhaltensannahme begrenzte Rationalität ist dadurch gekennzeichnet, dass in einer Welt mit Transaktionskosten und unvollkommener Voraussicht die Wirtschaftssubjekte nicht in der Lage sind, alle entscheidungsrelevanten Informationen $\mathrm{zu}$ erlangen und $\mathrm{zu}$ verarbeiten (Williamson 1990a, S. 52). Entscheidungen werden somit vor dem Hintergrund eines eingeschränkten Informationsstandes subjektiv rational getroffen, können jedoch zu objektiv begrenzt rationalen Ergebnissen führen (Simon 1961, S. 196-198). ${ }^{113}$

\section{Agrarverwaltung}

Im Rahmen einer Ausschreibung existieren staatlicherseits anfangs in der Regel zwei, langfristig drei Entscheidungsparameter. Kurzfristig - bei der erstmaligen Nutzung eines Ausschreibungsverfahrens - und in allen weiteren Ausschreibungsrunden sind dies das jeweilige ökonomische und ökologische Zuschlagskriterium; in der hier betrachteten Ausschreibung folglich der Preis pro Hektar und die Einstufung in die Kategorie der ökologischen Güter Grünland I, II oder III. Langfristig und frühestens nach Abschluss der ersten Ausschreibungsrunde kommt mit dem Wissen über die tatsächlich erbrachte ökologische Leistung die Reputation der Landwirte als drittes Entscheidungskriterium hinzu.

Hinsichtlich des ökonomischen Entscheidungsparameters besteht für die Agrarverwaltung eine Informationsasymmetrie dahingehend, dass ihr zum Zeitpunkt der Zuschlagserteilung die tatsächlichen Kosten der landwirtschaftlichen Betriebe nicht bekannt sind und eine Entscheidung ausschließlich orientiert an der Höhe des Angebotspreises erfolgt.

Im Gegensatz zu einer Einheitsprämie ist die Informationsasymmetrie hier jedoch geringer einzuschätzen, da von den individuellen Angebotspreisen Informationen zur Höhe der tatsächlichen Kosten zu erwarten sind. Nichtsdestoweniger trifft die Agrarverwaltung noch immer eine begrenzt rationale Entscheidung, da rational

\footnotetext{
${ }^{113}$ Siehe hierzu auch ausführlich Abschnitt 4.3.1 dieser Arbeit.
} 
anhand der zur Verfügung stehenden Informationen - den Angebotspreisen pro Hektar - entschieden wird, diese Informationen jedoch nicht vollständig sind. Zum Abbau der Informationsasymmetrie besteht grundsätzlich die Möglichkeit, durch die Erzeugung einer Konkurrenzsituation $\mathrm{zu}$ erreichen, dass überhöhte Angebote unterbleiben und eine größtmögliche Kostenorientierung bei der Kalkulation des Angebotspreises erreicht wird.

Ebenfalls besteht bei der ökologischen Bewertung der Angebote eine Informationsasymmetrie zu Lasten der staatlichen Entscheidungsträger. Zum Zeitpunkt der Zuschlagserteilung können sie die tatsächliche ökologische Qualität der Fläche nicht beurteilen und die Einstufung erfolgt anhand der angebotenen ökologischen Qualität. Zur Verbesserung der Informationsgrundlage besteht für den Staat langfristig die Möglichkeit des Lernens dahingehend, dass die tatsächliche Leistungsfähigkeit der Landwirte und die ökologische Qualität der Fläche in die Entscheidung einbezogen werden.

Als Folge der sowohl hinsichtlich des ökonomischen als auch des ökologischen Zuschlagskriteriums asymmetrischen Informationsverteilung $\mathrm{zu}$ Lasten der Agrarverwaltung kann es bei der Zuschlagserteilung in unterschiedlich hohem Maß zu suboptimalen Vertragsabschlüssen kommen.

Zum einen, da durch Unkenntnis der tatsächlichen Produktionskosten ein definierter ökologischer Nutzen zu einem zu hohen Preis nachgefragt wird. Zum anderen, da Angebote angenommen werden, die hinsichtlich des ökologischen Nutzens unzutreffend hoch eingestuft sind oder nicht erfüllt werden und dazu führen, dass Finanzmittel bis zum Ablauf des Ausschreibungsverfahrens gebunden werden, ohne einen positiven ökologischen Effekt zur Folge zu haben. Für eine objektiv rationale Entscheidung und einen optimalen Einsatz finanzieller Mittel wäre eine vollständige Überwindung dieser Informationsasymmetrie notwendig, so dass der Umfang der Informationsasymmetrie als Maß der begrenzten Rationalität dienen kann.

\section{Landwirte}

Die Entscheidungssituation eines teilnehmenden Landwirts ist sowohl durch eine ausschreibungsspezifische als auch eine der landwirtschaftlichen Produktion generell zugrunde liegende Unsicherheit geprägt. Diese führt dazu, dass die Angebotsabgabe 
nicht unter Berücksichtigung aller relevanten Informationen und demzufolge begrenzt rational erfolgt.

Einerseits besteht hinsichtlich der ökonomischen Angebotskomponente die Situation, dass die exakten Kosten in der Regel erst nach der Leistungserbringung bekannt sind und die Kalkulation des Angebotspreises mit einer entsprechenden Unsicherheit behaftet ist, die dazu führen kann, dass der Angebotspreis und somit die Honorierung zu gering ausfällt.

Zudem liegt für die Landwirte bis zum Zeitpunkt der Angebotsabgabe eine unvollständige Information dahingehend vor, dass sowohl die tatsächliche Höhe des zur Verfügung stehenden Budgets als auch die jeweiligen Angebotspreise der konkurrierenden Landwirte unbekannt sind.

Bezüglich der ökologischen Angebotskomponente, und somit der im Rahmen einer Ergebnisorientierung zu erbringenden Umweltleistung, besteht durch die in der Regel nicht oder nur in geringem Maß vorhersehbaren Umwelteinflüsse speziell bei der Förderung und dem Erhalt pflanzlicher Artenvielfalt ein Produktionsrisiko. Infolgedessen kann es durch die zum Zeitpunkt der Angebotsabgabe nicht in jedem Fall als sicher anzusehende Erbringung der Umweltleistungen zu der Situation kommen, dass eine Honorierung nach der erfolglosen Kontrolle unterbleibt und je nach Konzeption des Ausschreibungsverfahrens zudem eine Sanktionierung erfolgt.

Zusammenfassend ist die maßgeblich bis zum Vertragsabschluss relevante begrenzte Rationalität der Entscheidungsfindung sowohl für die Agrarverwaltung als auch die Landwirte als stark ausgebildet anzusehen.

\subsubsection{Opportunismus}

Die Verhaltensannahme des Opportunismus charakterisiert die Möglichkeit einer Verfolgung von Eigeninteresse unter Zuhilfenahme von strategischem und nicht vertragskonformem Verhalten. Dieses kann bewusst zu Lasten eines Transaktionspartners erfolgen und beispielsweise die gezielt unvollständige oder verfälschte Wiedergabe von Informationen umfassen (Williamson 1990a, S. 54-56). Hinsichtlich des Zeitpunkts eines Vertragsabschlusses ist außerdem zwischen Ex- 
ante-Opportunismus und Ex-post-Opportunismus zu unterscheiden (Richter und Furubotn 2003, S. 587). ${ }^{114}$

\section{Agrarverwaltung}

Anreize für Ex-ante-Opportunismus existieren für die Vergabestelle dahingehend, dass ein Interesse an möglichst geringen Angebotspreisen vorhanden ist, die sich exakt an den jeweiligen landwirtschaftlichen Produktionskosten orientieren. Jedoch besteht durch die Informationsasymmetrie zu Lasten der Agrarverwaltung eine Unkenntnis der Höhe dieser Produktionskosten, was dazu führt, dass bis zum Zeitpunkt der Zuschlagserteilung und des Vertragsabschlusses theoretisch ein grundsätzlicher Anreiz zu erkennen ist, diese Informationsasymmetrien auch durch Zuhilfenahme von opportunistischem Verhalten abzubauen oder zumindest zu minimieren.

Der Handlungsspielraum beschränkt sich auf die Wahl des Ausschreibungsdesigns und gezielter - gegebenenfalls nicht zutreffender - Informationen über die Begrenztheit und Höhe des zur Verfügung stehenden Ausschreibungsbudgets. Hierbei kann der Versuch unternommen werden, eine größere Konkurrenzsituation als tatsächlich vorhanden ist zu induzieren und so zu erreichen, dass die Landwirte keine überhöhten Angebotspreise abgeben.

Es wird deutlich, dass die Anreize für ein staatliches opportunistisches Verhalten speziell im Rahmen der erstmaligen Durchführung eines Ausschreibungsverfahrens theoretisch grundsätzlich vorhanden sind, da hier die gegebenen Informationen seitens der Landwirte noch nicht überprüft werden können.

Jedoch ist zu beachten, dass sowohl bei einer erstmaligen Ausschreibung als auch langfristig durch die kommunizierten Informationen die Anreize der Landwirte zu einer Teilnahme am Ausschreibungsverfahren verringert werden können und in der Praxis vor allem eine rechtssichere Abwicklung seitens der Agrarverwaltung zu unterstellen ist. Daher ist zu erwarten, dass die praktischen Möglichkeiten für staatlichen Ex-ante-Opportunismus vernachlässigt werden können. Die notwendige periodische Variation des Ausschreibungsdesigns und im Zuge dessen der Versuch ein Lernen und Preisabsprachen der Landwirte zu minimieren ist hier freilich nicht angesprochen, da es nicht als Opportunismus betrachtet werden kann.

\footnotetext{
${ }^{114}$ Siehe dazu ausführlich Abschnitt 4.3.2 der vorliegenden Arbeit.
} 
Nach der Zuschlagserteilung und folglich nach dem Vertragsabschluss besteht staatlicherseits kein Spielraum, denn die Handlungsmöglichkeiten der Agrarverwaltung sind bis einschließlich der Kontrolle der vertraglich zugesicherten Umweltleistung und der Auszahlung an die Landwirte durch exakte Handlungsvorgaben eindeutig festgelegt. Die Berücksichtigung eines Ex-postOpportunismus seitens der Agrarverwaltung entfällt daher im Rahmen eines Ausschreibungsverfahrens für Umweltleistungen.

\section{Landwirte}

Bis zum Zeitpunkt der Angebotsabgabe ist für die Landwirte ein Anreiz zu Ex-anteOpportunismus dahingehend vorhanden, durch die Abgabe eines hohen Angebotspreises die staatliche Unkenntnis der tatsächlichen Kosten auszunutzen und eine möglichst hohe Honorierung zu realisieren. Dazu existiert innerhalb eines Ausschreibungsverfahrens die Möglichkeit in Form eines überhöhten Angebotspreises implizit verzerrte oder nicht zutreffende Informationen zu den tatsächlichen Produktionskosten und der Leistungsfähigkeit eines landwirtschaftlichen Betriebs zu geben.

Jedoch besteht durch die Konkurrenzsituation um begrenzte Finanzmittel das Risiko, dass mit einem steigenden Angebotspreis die Zuschlagswahrscheinlichkeit sinkt und je nach Höhe des zur Verfügung stehenden Ausschreibungsbudgets überhöhte Angebotspreise dazu führen können, keinen Zuschlag $\mathrm{zu}$ erhalten. Diese geschilderten Anreize zu überhöhten Preisen liegen vor, wenn durch den Landwirt eine tatsächliche Teilnahme an einem Agrarumweltprogramm angestrebt wird.

Eine weitere Ausprägung von Opportunismus ist dahingehend denkbar, dass kein Anreiz besteht, tatsächlich an einem Programm teilzunehmen, sondern es ausschließlich das Ziel ist, innerhalb der Ausschreibungsregion finanzielle Mittel zu binden, so dass sie anderen Landwirten nicht zur Verfügung stehen. Somit würden keine $\mathrm{zu}$ hohen, sondern möglichst niedrige Angebotspreise abgegeben um infolgedessen die Wahrscheinlichkeit einer Zuschlagserteilung zu erhöhen.

Ein derartiger Anreiz ist jedoch nur im Rahmen der erstmaligen Durchführung eines Ausschreibungsverfahrens denkbar. Nach Abschluss der Ausschreibung kann seitens der Agrarverwaltung die tatsächliche Leistungsfähigkeit eines Landwirts erkannt werden und als negative Reputation Eingang in zukünftige Angebotsbewertungen finden. Je nach Umfang und Ausprägung möglicher über eine Nichtauszahlung 
hinausgehender Sanktionen ist zudem zu erwarten, dass mit steigenden Sanktionen der Anreiz zu opportunistischem Verhalten zurückgeht.

Nachdem eine Fläche in ein Programm genommen wurde, sind für den Landwirt grundsätzlich denkbare Ansatzpunkte für Ex-post-Opportunismus hinsichtlich der Erbringung der Umweltleistung zu erkennen. Ein Anreiz besteht, sich nicht vertragskonform zu verhalten und keinerlei Anstrengungen zur Produktion einer Umweltleistung vorzunehmen, jedoch die finanzielle Honorierung zu realisieren. Hier sind Handlungen vorstellbar, die Kontrolle durch eine ungenaue Schlagabgrenzung oder eine bewusst nicht repräsentative Wahl der Kontrollparzelle zu erschweren.

Praktisch erscheint dies jedoch wenig realistisch und für den Fall einer Kontrolle ist zu erwarten, dass eine nicht erbrachte Umweltleistung erkannt und entsprechend sanktioniert wird. Somit kann ein Ex-post-Opportunismus des Landwirts nahezu ausgeschlossen werden und eine mögliche Sanktionierung nicht vertragskonformen Verhaltens ist durch die Wahrscheinlichkeit einer Kontrolle bestimmt. Neben der Kontrollwahrscheinlichkeit ist zu erwarten, dass mit einer über die Nichtauszahlung hinausgehenden Sanktion ebenfalls eine Reduzierung opportunistischen Verhaltens der Landwirte nach Vertragsabschluss einhergeht.

Demzufolge zeigen die Ausführungen zur Ausprägung des Opportunismus ein gegenüber der begrenzten Rationalität abweichendes und weniger eindeutiges Bild. Für die Agrarverwaltung kann praktisch von keinen Möglichkeiten zu Opportunismus ausgegangen werden, da eine vertragskonforme und Rechtssicherheit gebietende Abwicklung seitens dieser staatlichen Institution unterstellt werden muss. Für Landwirte sind grundsätzlich Anreize zu opportunistischem Verhalten in Form der Weitergabe unzutreffender oder ungenauer Informationen zu erkennen.

Hierbei ist zu berücksichtigen, dass auch Williamson die Ausprägung von Opportunismus nicht permanent unterstellt, sondern lediglich jederzeit die Möglichkeit eines entsprechenden Verhaltens einbezieht, was einer Absicherung bedarf (Williamson 1990a, S. 55). Doch nichtsdestoweniger haben die obigen Ausführungen gezeigt, dass entsprechende Anreize seitens der Agrarverwaltung faktisch nicht gegeben sind und derartige Aspekte einer staatlichen Institution in dieser Form bislang keine Berücksichtigung gefunden haben. 


\subsection{Dimensionen von Transaktionen}

Nachdem die Vertragsbeziehung zwischen Agrarverwaltung und Landwirt hinsichtlich der Verhaltensannahmen betrachtet wurde, wird die Analyse im Nachfolgenden um die Dimensionen von Transaktionen erweitert. Dabei werden die Faktorspezifität, die Unsicherheit ${ }^{115}$ und die Häufigkeit jeweils kurz skizziert und auf die Transaktionsbeziehung innerhalb eines Ausschreibungsverfahrens angewendet.

\subsubsection{Faktorspezifität}

Die Dimension Faktorspezifität analysiert die Frage, welchen Einfluss die Spezifität einer Transaktionsbeziehung auf ihre Stabilität und Absicherung ausübt (Williamson 1990a, S. 62-63; Richter und Furubotn 2003, S. 153). Im Zuge dessen wird mit einer steigenden Spezifität der Vertragsbeziehung eine zunehmende Bindung der Vertragspartner unterstellt und der Prozess von einer Konkurrenzsituation hin zu einer bilateralen Abhängigkeit der Transaktionspartner analog zu Williamson als fundamentale Transformation bezeichnet (Williamson 1990b, S. 70).

Im Kontext einer Ausschreibung wird die Spezifität der Transaktionsbeziehung hinsichtlich der jeweiligen potentiellen Transaktionspartner wie auch der angebotenen und angenommenen Flächen im Mittelpunkt stehen. ${ }^{116}$

Hierzu wird nun der Gedanke der fundamentalen Transformation ${ }^{117}$ einbezogen und nachfolgend auf den Bereich der Ausschreibung von Umweltleistungen angewendet. Im Zuge dessen gilt es zunächst die Frage zu untersuchen, ob eine anfänglich hohe Teilnehmerzahl an einem Bietprozess auch in der Folge bestehen bleibt oder ob es bedingt durch transaktionsspezifische Investitionen zu einer Reduzierung der Teilnehmerzahl und infolgedessen einer Einschränkung der Konkurrenzsituation kommt (Williamson 1990a, S. 70-72).

Für den Fall, dass keine transaktionsspezifischen Investitionen getätigt wurden, kann eine Konkurrenzsituation grundsätzlich auch für weitere Verhandlungsrunden

\footnotetext{
115 Um ihre praktische Relevanz aufzuzeigen, findet die Dimension Unsicherheit hierbei, im Gegensatz zu den Ausführungen in Abschnitt 4.5, Berücksichtigung.

${ }^{116}$ Für eine ausführliche Einführung der Dimension Faktorspezifität siehe auch Abschnitt 4.4.1 dieser Arbeit.

${ }^{117}$ Siehe hierzu ebenfalls Abschnitt 4.4.4 dieser Arbeit.
} 
bestehen bleiben, da keiner der Akteure entsprechende Vorteile gegenüber Mitkonkurrenten aufbauen konnte (Williamson 1990a, S. 70).

Wurden jedoch im Verlauf der Transaktionsbeziehung spezifische Investitionen vorgenommen, so hat dies zur Konsequenz, dass vormals erfolgreiche Bieter sich für zukünftige Bietprozesse eine gegenüber ihren Mitkonkurrenten bessere Verhandlungsposition schaffen konnten. In der Folge existiert nicht mehr die ursprüngliche Konkurrenzsituation, sondern die Anreizstrukturen und spezifischen Bindungen der Vertragspartner haben sich verändert. Demzufolge wird ein Wechsel der Transaktionspartner durch im Zuge dessen zu erwartende finanzielle Einbußen bzw. hohe Transaktionskosten erschwert oder verhindert und die daraus resultierende Situation wird von Williamson in ihrer extremsten Ausprägung als bilaterale oder einseitige Monopolsituation charakterisiert (Williamson 1990a, S. 70-71).

Die fundamentale Transformation kennzeichnet somit diesen Prozess einer Vertragsbeziehung unter dem Gesichtspunkt ihrer Veränderung von einer ex-ante unspezifischen hin zu einer ex-post spezifischen Transaktionsbeziehung und sich daraus ergebenden Konsequenzen für zukünftige Vertragsverhandlungen.

Die Ausgangssituation innerhalb der Ausschreibung ist dadurch gekennzeichnet, dass die Agrarverwaltung als einziger Anbieter eines spezifischen Agrarumweltprogramms und alleiniger Nachfrager dar darin ausgeschriebenen Umweltleistung auftritt. Dem steht als Anbieter der ökologischen Leistung eine Vielzahl von Landwirten gegenüber und folglich ist die Angebotsseite zu Beginn einer erstmaligen Ausschreibung durch eine umfassende Konkurrenzsituation gekennzeichnet.

Im sich anschließenden Prozess der Zuschlagserteilung erhalten je nach der Höhe des zur Verfügung stehenden Budgets in der Regel nicht alle Landwirte für ihre angebotenen Flächen einen Zuschlag. Die Landwirte, welche einen Zuschlag erhalten haben, werden nun Aktivitäten zur Produktion der Umweltleistung erbringen und im Zuge dessen transaktionsspezifische Investitionen tätigen. Wird die ökologische Leistung innerhalb der Vertragslaufzeit erbracht, kann es in der Folge sowohl aus Sicht der Agrarverwaltung als auch seitens der Landwirte zu Lock-in-Effekten in Form von Anreizen zu einer spezifischen Bindung an den Transaktionspartner kommen. 
Die daraufhin im Kontext einer wiederholten Ausschreibungsrunde zentrale Frage ist die Entscheidung, wie nach dem Ende der Vertragslaufzeit bereits vertraglich gebundene Flächen - auf denen die Umweltleistung nachweislich erbracht wurde und potentielle neue Angebote im Zuge des erneuten Bietprozesses und der sich anschließenden Zuschlagserteilung behandelt werden. Diesbezüglich sind grundsätzlich die beiden nachfolgenden Möglichkeiten denkbar.

\section{Einerseits kann zwischen bereits erfolgreich in einem Programm befindlichen} und neu angebotenen Flächen keine Unterscheidung vorgenommen werden, so dass alle Angebote erneut gleichberechtigt und hinsichtlich der identischen Kriterien um einen Zuschlag konkurrieren.

Im Zuge dessen ist langfristig die Aufrechterhaltung einer Konkurrenzsituation gegeben und zudem bleibt ein Programm uneingeschränkt auch für die Landwirte offen, welche bislang noch nicht an dem Agrarumweltprogramm teilgenommen haben. Demgegenüber kann es zu der Situation kommen, dass bislang nachweislich die Umweltkriterien erfüllende Flächen keinen erneuten Zuschlag erhalten und stattdessen Angebote über deren tatsächliche Umweltqualität noch keine Aussage möglich ist, vertraglich gebunden werden. Damit einher geht für die Agrarverwaltung eine zunehmende Unsicherheit bezüglich der zu erreichenden Umweltleistung eines Programms. Für die vormals vertraglich gebundenen und keinen erneuten Zuschlag erhaltenden Landwirte stellt sich die Situation ein, dass sie durch die mit der Teilnahme an einem Agrarumweltprogramm vollzogene Umstellung ihrer Flächennutzung zukünftig unter Umständen keine Honorierung erhalten können, was sowohl einen entsprechenden Einkommensrückgang als auch langfristige Akzeptanzeinbußen mit sich bringen kann.

\section{Die zweite denkbare Vorgehensweise ist eine Ungleichbehandlung der in der} auslaufenden Ausschreibung bereits vertraglich gebundenen erfolgreichen Angebote und neuer angebotener Flächen.

Dabei besteht die Möglichkeit, die in der Vergangenheit erfolgreichen Angebote unmittelbar erneut in ein Programm aufzunehmen beziehungsweise sie im Rahmen der Zuschlagserteilung anhand spezifischer Kriterien bevorzugt zu behandeln und ihnen eine höhere Zuschlagswahrscheinlichkeit zukommen zu lassen. Neue Angebote können dann lediglich im Umfang eines noch zur Verfügung stehenden 
Budgets oder durch die Aussicht auf eine umfassende Erfüllung ökologischer und ökonomischer Kriterien angenommen werden.

Für die Agrarverwaltung ist damit insbesondere der Anreiz verbunden, die ökologischen Ziele eines Agrarumweltprogramms erneut möglichst sicher zu erreichen. Demgegenüber besteht aus ökonomischer Sicht die Problematik, dass durch eine mangelnde Konkurrenzsituation und Wettbewerbsverzerrungen auf Seiten der eine Umweltleistung anbietenden Landwirte Effizienzeinbußen und eine eingeschränkte Flexibilität der Zuschlagserteilung zu erwarten sind. Für die Landwirte, welche bereits innerhalb einer vorherigen Ausschreibungsrunde sowohl eine Umstellung der Bewirtschaftung vollzogen als auch entsprechende spezifische Investitionen getätigt haben, ist positiv $\mathrm{zu}$ bewerten, dass diese Transaktionsbeziehung mit einer hohen Wahrscheinlichkeit beibehalten wird. Demgegenüber ist für potentiell erstmals an einer Angebotsabgabe interessierte Landwirte in der Regel eine erschwerte Möglichkeit einer Zuschlagserteilung und damit verbunden eine geringe Ausprägung von Anreizen zur Erbringung einer Umweltleistung zu erwarten.

Zusammenfassend zeigen sich die hohe Faktorspezifität und die praktische Relevanz der fundamentalen Transformation im Kontext der Ausschreibung von Umweltleistungen vor allem im Hinblick auf die Zuschlagserteilung im Rahmen eines wiederholten Ausschreibungsverfahrens. Diesbezüglich ist zu erkennen, dass beide zu unterscheidenden Vorgehensweisen zu Problemen führen können, wobei aus Sicht der Agrarverwaltung jeweils eine eher von ökologischen und eine eher von ökonomischen Zielen geprägte Ausrichtung denkbar ist. Jedoch kann an dieser Stelle keine abschließende Wertung getroffen werden und das praktische Vorgehen ist letztlich einerseits von der jeweiligen Umweltleistung mit den entsprechenden Rahmenbedingungen abhängig und nicht zuletzt als eine politische Entscheidung anzusehen.

Für die in der vorliegenden Arbeit durchgeführten Ausschreibungsverfahren wurde bei der Zuschlagserteilung die Ausgestaltung einer Gleichbehandlung aller Angebote gewählt, wobei auf diese Aspekte erneut anhand der Diskussion der praktischen Erprobung in Kapitel Sieben eingegangen wird. 


\subsubsection{Unsicherheit}

Bei einem Ausschreibungsverfahren herrscht je nach Art und Ausgestaltung in verschiedenem Maß Unsicherheit über zukünftige für die Transaktionsbeziehung relevante Aspekte. Hinsichtlich der Möglichkeit einer Beeinflussung durch die Transaktionspartner wird zwischen exogenen Risiken und Verhaltensunsicherheiten unterschieden. Exogene Risiken sind zum Zeitpunkt des Vertragsabschlusses nicht absehbare und von den Vertragspartnern nicht beeinflussbare Unsicherheiten. Verhaltensunsicherheiten hingegen sind als bewusste Fehlinformationen oder nicht vertragskonformes Verhalten analog zu Opportunismus anzusehen und stellen für den jeweiligen Transaktionspartner eine Unsicherheit dar (Williamson 1990a, S. 6566; Richter 1994, S. 18-19). Eine besondere Bedeutung kommt der Dimension Unsicherheit innerhalb einer durch eine hohe Spezifität gekennzeichneten Transaktionsbeziehung zu, da hier ein Abweichen von der vertraglichen Regelung mit erheblichen Anpassungskosten verbunden ist. ${ }^{118}$

\section{Agrarverwaltung}

Für die Agrarverwaltung besteht bis zur Zuschlagserteilung eine Unsicherheit dahingehend, dass die tatsächliche Qualität der Angebote nicht überprüft werden kann und eine begrenzt rationale Entscheidung getroffen wird, welche zur Folge haben kann, dass zu hohe Preise akzeptiert werden.

Nach der Zuschlagserteilung herrscht sowohl eine Verhaltensunsicherheit als auch eine Unsicherheit hinsichtlich nicht beeinflussbarer Umweltfaktoren vor.

Die Verhaltensunsicherheit beinhaltet die Frage, ob die Umweltleistung durch den Transaktionspartner im Rahmen der Vertragslaufzeit wie vereinbart erbracht wird, was sowohl durch seine tatsächliche Leistungsfähigkeit als auch die Ausprägung eines möglichen opportunistischen Verhaltens beeinflusst wird. Als Folge einer nicht erbrachten Umweltleistung entsteht die Situation, dass staatliche Mittel gebunden wurden, ohne einen ökologischen Nutzen zu erzielen. Für die Agrarverwaltung bietet sich eine Einflussmöglichkeit lediglich bei der Wahl des Ausschreibungsdesigns und dem Versuch, durch eine entsprechende Androhung von Sanktionen einmalig

\footnotetext{
118 Grundsätzlich ist festzuhalten, dass die Verhaltensannahmen maßgeblich durch die vorhandenen Unsicherheiten geprägt sind und eine entsprechende Argumentation umfassend bereits dort erfolgt ist. Infolgedessen werden diese Aspekte nachfolgend kurz betrachtet.
} 
Anreize zu vertragskonformem Verhalten zu implementieren und das Ausmaß der Verhaltensunsicherheit zu reduzieren.

Bezüglich der durch potentielle exogene Risiken vorherrschenden Unsicherheit besteht staatlicherseits keine Einflussmöglichkeit und als Folge kann es - durch Umwelteinflüsse oder höhere Gewalt - dazu kommen, dass ein angestrebter ökologischer Nutzen nicht erreicht wird.

\section{Landwirte}

Die größte Unsicherheit innerhalb eines Ausschreibungsverfahrens ist für den Inhaber eines landwirtschaftlichen Betriebs vor dem Vertragsabschluss dadurch gegeben, dass es bis zur Zuschlagserteilung nicht sicher ist, ob das Angebot angenommen wird und für die betreffende Fläche eine zukünftige Zahlung realisiert werden kann. Dabei ist grundsätzlich zu erwarten, dass mit einem steigenden Angebotspreis die Wahrscheinlichkeit einer Zuschlagserteilung sinkt.

Nachdem der Vertrag abgeschlossen ist, besteht für den Landwirt hinsichtlich seiner Erfüllung durch die Agrarverwaltung keine Verhaltensunsicherheit, da ihn der Staat als sicherer Vertragspartner vollständig und rechtssicher erfüllen wird. Eine große Bedeutung hat jedoch die nicht beeinflussbare Unsicherheit hinsichtlich eines beispielsweise durch Umwelteinwirkungen bestehenden Produktions- und Ausfallrisikos.

In einer über die Vertragslaufzeit hinausgehenden Betrachtung ist für die Landwirte zudem eine Unsicherheit dahingehend $\mathrm{zu}$ erkennen, dass es nicht als sicher angesehen werden kann, ob ein spezifisches Agrarumweltprogramm seitens der Agrarverwaltung noch einmal angeboten wird und eine erneute Zuschlagserteilung erfolgt.

Demzufolge kann dahingehend von einer für die transaktionskostenökonomische Analyse untergeordneten Rolle der Unsicherheit gesprochen werden, als dass sie nicht beeinflussbar ist und auch hier für die weiteren Betrachtungen als gegeben angesehen wird. 


\subsubsection{Häufigkeit}

Abschließend wird die Dimension Häufigkeit betrachtet, welche sich mit dem Einfluss einmalig, gelegentlich oder regelmäßig durchgeführter Transaktionen auf deren Effizienz und Ausgestaltung beschäftigt (Williamson 1990a, S. 69). ${ }^{119}$

Im Rahmen der Analyse von Ausschreibungen in Agrarumweltprogrammen wird hierbei die Vertragslaufzeit für eine in das Programm genommene Fläche betrachtet. In der praktischen Ausgestaltung von Agrarumweltprogrammen ist die Vertragslaufzeit in der Regel auf fünf Jahre festgelegt, wobei jährlich stichprobenartige Kontrollen der vertraglichen Verpflichtungen erfolgen und die Auszahlungen nach einer erfolgreichen Kontrolle ebenfalls jährlich vorgenommen werden. ${ }^{120}$ Somit können Flächen erst nach Ablauf der gesamten Vertragslaufzeit erneut angeboten werden.

\section{Agrarverwaltung}

Für die Vergabestelle besteht erstmals nach Abschluss der Ausschreibungsrunde die Möglichkeit, eine Beurteilung hinsichtlich der tatsächlichen Vertragserfüllung vorzunehmen. Langfristig eröffnet sich für den Fall einer Wiederholung der Ausschreibung einer identischen Umweltleistung der Ansatzpunkt sowohl die Leistungsfähigkeit als auch die Produktionskosten teilnehmender Landwirte zunehmend genauer zu identifizieren. Im Zuge dessen kann eine Reputation der Landwirte aufgebaut und in die Angebotsbewertung einbezogen werden, welche dazu beiträgt, einerseits bestehende Informationsasymmetrien $\mathrm{zu}$ reduzieren und andererseits im Zuge einer Sanktionierung bei weiteren Zuschlagserteilungen Anreize zu opportunistischem Verhalten der Landwirte zu verringern.

Ebenso kann auf der Grundlage der abgeschlossenen Ausschreibungsrunden durch Anpassungen des Ausschreibungsdesigns auf unerwünschte Mitnahmeeffekte oder Preisabsprachen reagiert und so eine Verbesserung der ökologischen und ökonomischen Zielgenauigkeit einer Agrarumweltmaßnahme erzielt werden.

Darüber hinaus sind im Zuge der Nutzung von identischen Verträgen und der langfristigen Beibehaltung von Vertragsbeziehungen mit Landwirten

\footnotetext{
${ }^{119}$ Siehe hierzu auch die Ausführungen in Abschnitt 4.4.3 der vorliegenden Arbeit.

${ }^{120}$ Die Vertragslaufzeit beträgt im Forschungsprojekt ein Jahr oder weniger; siehe dazu ausführlich Kapitel Sieben dieser Arbeit.
} 
transaktionskostenmindernde Effekte der Abwicklung von Agrarumweltprogrammen zu erwarten.

\section{Landwirte}

Für die Landwirte eröffnet sich mit einer zunehmenden Anzahl von Ausschreibungsrunden die Möglichkeit, aus der Bewertung ihrer vergangenen Angebote - vor allem hinsichtlich der Angebotspreise, der Zuschlagsgrenzen, der tatsächlichen Produktionskosten und der zum Kontrollzeitpunkt erbrachten Umweltleistung - zu lernen und Erwartungen für zukünftige Angebotsabgaben zu bilden.

Zudem ist grundsätzlich zu erwarten, dass es für die Landwirte im Verlauf sich wiederholender Ausschreibungsverfahren $\mathrm{zu}$ transaktionskostenmindernden Lerneffekten in der Form kommt, dass Unsicherheiten reduziert werden können und die Angebotsabgabe einen geringeren Zeitaufwand erforderlich macht. Jedoch sind hierbei die durch eine Mindestvertragslaufzeit von fünf Jahren festgelegten Zeiträume bis zu einer neuen Teilnahme relevant, die eine erneute Angebotsabgabe unter identischen Rahmenbedingungen unwahrscheinlich machen.

Zusammenfassend ist zu erkennen, dass mit einer zunehmenden Wiederholung der Ausschreibung einer identischen Umweltleistung sowohl seitens der Agrarverwaltung als auch seitens der Landwirte die grundsätzliche Möglichkeit besteht, die Ergebnisse und Erfahrungen vergangener Ausschreibungsrunden in die jeweils aktuellen Entscheidungsprozesse einzubeziehen. Im Zuge dessen sind bei der wiederholten Durchführung einer identischen Ausschreibung zudem für die Agrarverwaltung und für die Landwirte transaktionskostenmindernde Effekte zu erwarten.

\subsection{Beherrschung und Überwachung von Transaktionen}

Auf der Grundlage der obigen Analyse der Verhaltensannahmen und der Dimensionen von Transaktionen innerhalb eines Ausschreibungsverfahrens zur Honorierung ökologischer Leistungen der Landwirtschaft, wird im Folgenden eine optimale Beherrschungsstruktur diskutiert. Dazu werden die in der Transaktionskostenökonomik üblicherweise unterschiedenen vier 
Beherrschungsstrukturen Marktkontrolle, dreiseitige Beherrschungsstruktur, zweiseitige Beherrschungsstruktur und einseitige Beherrschungsstruktur hinsichtlich ihrer Anwendbarkeit beurteilt.

\section{Marktkontrolle}

Die Marktkontrolle ist dann als angemessen anzusehen, wenn über einen funktionierenden Markt und bei gegebenen rechtlichen Rahmenbedingungen unspezifische Transaktionen gelegentlich oder regelmäßig abgewickelt werden. Demzufolge wird durch die Möglichkeit zu relativ geringen Kosten opportunistisches Verhalten zu sanktionieren und den Transaktionspartner $\mathrm{zu}$ wechseln eine bestmögliche Absicherung von Transaktionen ermöglicht (Williamson 1990a, S. 83; Richter und Furubotn 2003, S. 196-197).

Im Fall eines Ausschreibungsverfahrens für Umweltleistungen liegt keine funktionierende zweiseitige Marktsituation mit gleichberechtigten und strukturell ähnlichen Transaktionspartnern vor. Zudem sind die innerhalb von Agrarumweltprogrammen abgeschlossenen Verträge weder als kurzfristig noch als vollständig zu charakterisieren, in denen die Identität der Akteure irrelevant ist. Somit besteht keine unspezifische Transaktionsbeziehung, die gelegentlich oder regelmäßig abgewickelt wird und eine beidseitige Möglichkeit opportunistisches Verhalten zu geringen Kosten durch die Suche eines neuen Transaktionspartners zu sanktionieren ist nicht gegeben. Infolgedessen ist die Beherrschungsstruktur einer reinen Marktkontrolle hier nicht zutreffend.

\section{Dreiseitige Beherrschungsstruktur}

Werden mittel- und hochspezifische Transaktionen nur einmalig oder gelegentlich durchgeführt, sind die Marktkräfte zur Absicherung dieser Transaktionen nicht ausreichend. Es bedarf daher der Einbeziehung einer neutralen dritten Institution, welche als Vermittlungs- oder Schiedsinstanz die Einhaltung der vertraglichen Vereinbarungen sicherstellt und gegenüber der Marktkontrolle die entscheidende Erweiterung innerhalb der dreiseitigen Beherrschungsstruktur darstellt (Williamson 1990a, S. 84-85; Richter und Furubotn 2003, S. 197).

Ebenso wie die Marktkontrolle unterstellt auch die dreiseitige Beherrschungsstruktur grundsätzlich die Existenz eines funktionierenden Marktes. Eine Übereinstimmung 
mit der innerhalb eines Ausschreibungsverfahrens vorherrschenden Situation wäre somit grundsätzlich nur dann gegeben, wenn die Agrarverwaltung und die Landwirte hinsichtlich der Ausprägung ihrer Verhaltensannahmen als strukturell ähnliche Transaktionspartner angesehen werden können, wobei die Agrarverwaltung sowohl einen Teil der Transaktionsbeziehung als auch eine neutrale Dritte Instanz darstellt, die durch die Bereitstellung des relevanten Rechtsrahmens die vertragskonforme Vertragserfüllung sicherstellt.

Dies ist im Rahmen der Ausschreibung nicht gegeben. Einerseits haben die Ausführungen zu den Verhaltensannahmen gezeigt, dass die Agrarverwaltung nicht als ein den Landwirten ähnlicher Transaktionspartner betrachtet werden kann und demzufolge keine Absicherung der Marktseite der Agrarverwaltung notwendig ist. Zudem kann die Agrarverwaltung nicht als gänzlich neutrale Institution angesehen werden kann, da sie die für die Transaktion relevanten Rahmenbedingungen als Transaktionspartner selbst geschaffen hat.

Als uneingeschränkt zutreffend sind demgegenüber auch für die Ausschreibung von Umweltleistungen - sowohl hinsichtlich der hohen Spezifität als auch der einmaligen oder gelegentlichen Durchführung der Transaktion - die Ausprägungen der Dimensionen der Faktorspezifität und der Häufigkeit anzusehen.

\section{Zweiseitige Beherrschungsstruktur}

Ein individuell ausgestalteter und in seiner Laufzeit in der Regel unbestimmter relationaler Vertrag stellt bei mittelspezifischen und regelmäßig wiederkehrenden Transaktionen die bestmögliche Absicherung dar. Innerhalb dieser zweiseitigen Beherrschungsstruktur können marktliche Anreize teilweise bestehen bleiben und gleichzeitig bei einer langfristigen Beibehaltung der Vertragsausgestaltung die Transaktionskosten gegenüber einer Marktkontrolle reduziert werden (Williamson 1990a, S. 85-88; Richter und Furubotn 2003, S. 197).

Der im Rahmen eines Ausschreibungsverfahrens abgeschlossene Vertrag ist ein individuell ausgestalteter zweiseitiger Vertrag. Jedoch ist dieser in der Regel nur für die jeweilige Vertragslaufzeit gültig und ein erneuter Abschluss zu gleichen Vertragsbedingungen ist unwahrscheinlich. Zudem fehlt auch hier die durch eine funktionierende Marktsituation induzierte grundsätzliche Existenz einer zweiseitigen Konkurrenzsituation strukturell ähnlicher Transaktionspartner. Somit ist eine 
zweiseitige Beherrschungsstruktur für Ausschreibungen sowohl hinsichtlich der Spezifität und der Häufigkeit der Transaktionsbeziehung als auch der Ausprägung des Opportunismus nicht zutreffend.

\section{Einseitige Beherrschungsstruktur (Hierarchie)}

Bei hochspezifischen und sich regelmäßig wiederholenden Transaktionen erfolgt ihre Absicherung bestmöglich durch eine vollständige vertikale Integration. Mit steigender Faktorspezifität geht die Anzahl der potentiellen Transaktionspartner zurück und die Anreize für einen Markttausch sinken durch eine zunehmende Gefahr opportunistischen Verhaltens, dem mit einer organisationsinternen Transaktionsabwicklung unter einer einheitlichen Anreizstruktur entgegnet werden kann (Williamson 1990a, S. 88; Richter und Furubotn 2003, S. 197-198).

Zutreffend ist für die Ausschreibung ökologischer Leistungen grundsätzlich die hohe Spezifität der Transaktionsbeziehung und für den Fall eines Vertragsabschlusses ebenfalls die grundsätzliche Vereinheitlichung der Interessen der Marktpartner in Form der Erfüllung einer spezifischen Umweltleistung; einerseits im Hinblick auf die Realisierung agrarumweltpolitischer Ziele und andererseits hinsichtlich einer finanziellen Honorierung. Dies ist jedoch nur für die einmalige Dauer der Vertragslaufzeit als zutreffend anzusehen. Danach erfolgt eine neue Ausschreibung der Umweltleistungen, welche als Bietprozess mit einer neuen Konkurrenzsituation einhergeht und an deren Ende es nicht als sicher angesehen werden kann, dass eine vormals vertraglich gebundene Fläche erneut und zu den identischen Konditionen in ein Programm genommen wird.

Für wiederholte Transaktionen, wie die hier zugrunde gelegte Ausgestaltung eines Ausschreibungsverfahrens, ist die einseitige Beherrschungsstruktur in Form einer mehrperiodigen vollständigen vertikalen Integration demzufolge nicht zutreffend.

\subsection{Zusammenfassende Diskussion}

Die bisherige Argumentation verdeutlicht, dass die Transaktionsbeziehung innerhalb eines Ausschreibungsverfahrens für Umweltleistungen durch eine von der traditionellen Betrachtungsweise innerhalb der Transaktionskostenökonomik abweichende Besonderheit gekennzeichnet ist. Demzufolge zeigt sich, dass keine der 
vier in der Regel unterschiedenen Ausgestaltungsformen für diese spezifische Transaktion als vollständig übereinstimmend anzusehen ist.

Die Marktkontrolle und die zweiseitige Beherrschungsstruktur sind sowohl hinsichtlich der unterstellten Ausprägung der Faktorspezifität und der Häufigkeit der Transaktionsbeziehung als auch dem für die Marktseite der Agrarverwaltung nicht zutreffenden Opportunismus gänzlich unzutreffend.

Eine teilweise Übereinstimmung mit der betrachteten Ausschreibung ist für die dreiseitige Beherrschungsstruktur und die einseitige Beherrschungsstruktur zu erkennen.

Die einseitige Beherrschungsstruktur trägt der hohen Spezifität der Transaktionsbeziehung innerhalb einer Ausschreibung für Umweltleistungen ebenso wie der grundsätzlich vereinheitlichten Anreizstruktur der Transaktionspartner mit dem Ziel der positiven Vertragserfüllung Rechnung. Hinsichtlich der Dimension der Häufigkeit unterstellt die einseitige Beherrschungsstruktur demgegenüber eine im Rahmen dieser Ausschreibung in der Praxis nicht zu erwartende regelmäßige Wiederholung einer identischen Transaktion über mehrere Ausschreibungsrunden hinweg und einer damit einhergehenden langfristigen Bindung der Vertragspartner. Innerhalb der dreiseitigen Beherrschungsstruktur ist sowohl im Hinblick auf die Ausprägung einer hohen Spezifität als auch der Häufigkeit der einmalig oder gelegentlich durchgeführten identischen Transaktionsbeziehung eine Übereinstimmung mit der Situation innerhalb eines Ausschreibungsverfahrens für Umweltleistungen der Landwirtschaft vorhanden. Als nicht zutreffend einzuschätzen ist hingegen die Verhaltensannahme des Opportunismus für die Agrarverwaltung als Nachfrager der Umweltleistung, da sie weder als den Landwirten strukturell identischer Transaktionspartner noch als gleichzeitige neutrale dritte Instanz angesehen werden kann.

Somit kann eine auf den gewonnenen Erkenntnissen aufbauende Argumentation erfolgen, welche eine Erweiterung des Analyserahmens auf den Spezialfall einer Ausschreibung vornimmt. Darüber hinaus wird das in dieser Arbeit gewählte Ausschreibungsdesign einbezogen und hinsichtlich seiner Einordnung in den Kontext der Transaktionskostenökonomik diskutiert. 


\section{Die Ausschreibung von Umweltleistungen als „einseitige Marktkontrolle“}

Um den Besonderheiten der Transaktionsbeziehung innerhalb einer Ausschreibung Rechnung zu tragen und den Analyserahmen zu erweitern, wird die in diesem Zusammenhang als einseitige Marktkontrolle bezeichnete Beherrschungsstruktur eingeführt.

Die Verhaltensannahmen sind hierbei in der Form ausgebildet, dass die begrenzte Rationalität für alle Transaktionspartner als gegeben unterstellt wird. Demgegenüber sind Anreize und Möglichkeiten für opportunistisches Verhalten durch die Einbeziehung einer staatlichen Institution als Nachfrager ausschließlich auf der Angebotsseite zu berücksichtigen.

Die Dimensionen der Transaktion sind bei einer als gegeben betrachteten Unsicherheit wie folgt ausgeprägt. Die Transaktionsbeziehung ist durch eine hohe Faktorspezifität gekennzeichnet, wobei eine für alle Vertragsbestandteile identische Transaktion - je nach praktischer Ausgestaltung der Ausschreibung - in der Regel einmalig abgewickelt wird.

Die Charakterisierung dieser einseitigen Beherrschungsstruktur verdeutlicht eine auf der dreiseitigen Beherrschungsstruktur aufbauende Argumentation. Demzufolge ist die entscheidende Weiterentwicklung hinsichtlich der Verhaltensannahme des Opportunismus zu sehen. Mittels der Einbeziehung eines staatlichen Akteurs als alleinigem und durch keine praktischen Möglichkeiten zu opportunistischem Verhalten gekennzeichnetem Nachfrager ist eine Absicherung durch eine Wettbewerbssituation auf der Nachfrageseite nicht möglich und nicht notwendig.

Es zeigt sich nunmehr die Übereinstimmung der abgeleiteten einseitigen Marktkontrolle mit dem innerhalb dieser Arbeit gewählten Ausschreibungsdesign einer simultanen Mehrgüterausschreibung mit Preis-Mengen-Angeboten und einer preisdiskriminierenden Zuschlagserteilung ${ }^{121}$ sowie der zugrunde liegenden Transaktionsbeziehung. Die Vergabestelle (hier die Universität Göttingen, wobei die analogen Schlüsse für die Agrarverwaltung zu ziehen sind) tritt für jede der simultan ausgeschriebenen drei Umweltleistungen der ökologischen Güter Grünland I, II und III gleichermaßen als alleiniger Anbieter eines Programms und alleiniger Nachfrager der Umweltleistungen auf. Die Landwirte stehen als potentielle Anbieter der Umweltleistungen in einer auf ihrer Marktseite ausgeprägten Konkurrenzsituation

\footnotetext{
${ }^{121}$ Siehe hierzu ausführlich Abschnitt 5.2.5 dieser Arbeit.
} 
um ein jeweils begrenztes Budget, wobei in der hier gewählten Ausgestaltung der Angebotspreis pro Einheit das zentrale Zuschlagskriterium ist.

Im Zuge dessen dienen die Vergabebedingungen als verbindlich zu erfüllender Rechtsrahmen, der keinen praktischen Spielraum für Opportunismus der Vergabestelle eröffnet und demzufolge keine Notwendigkeit einer entsprechenden Absicherung notwendig macht. Demgegenüber ist die Leistungserbringung durch den Landwirt sowohl bedingt durch nicht beeinflussbare Umwelteinflüsse als auch eine mangelnde Leistungsfähigkeit und Opportunismus gefährdet. Somit erfolgt grundsätzlich auch innerhalb einer Ausschreibung eine Marktkontrolle, jedoch lediglich auf der durch Opportunismus gefährdeten Marktseite der Landwirte als konkurrierende Anbieter einer Umweltleistung.

Im Hinblick auf die Verhaltensannahme der begrenzten Rationalität ist zu konstatieren, dass sie sowohl für die Agrarverwaltung als auch die Landwirte als mitunter stark ausgeprägt angesehen werden muss und ihre Relevanz der Transaktionskostenökonomik entsprechend ebenfalls innerhalb des Ausschreibungsverfahrens vorhanden ist. Dies ist vor allem durch eine zum Zeitpunkt des Vertragsabschlusses für beide Transaktionspartner vorherrschende Unsicherheit und einen eingeschränkten Informationsstand begründet, was dazu führt, dass auch hier in der Praxis zwangsläufig ein unvollständiger Vertrag geschlossen wird.

In diesem Kontext ist die ausgeschriebene Umweltleistung ebenso wie die damit verbundene Transaktionsbeziehung hinsichtlich ihrer Faktorspezifität aus Sicht beider Transaktionspartner als hochspezifisch anzusehen. Einerseits wird im Rahmen einer Vertragsdifferenzierung bezüglich der Angebotspreise jeweils ein individueller Vertrag geschlossen und die Bindung der finanziellen Mittel erfolgt seitens der Agrarverwaltung ausschließlich für diese spezifische Umweltleistung. Eine Nichterfüllung des Vertrags durch den Landwirt hätte demzufolge die Auswirkung, dass Finanzmittel gebunden werden, ohne dass damit ein erwünschter ökologischer Nutzen erreicht wird und dies nicht unmittelbar durch ein anderes Programm ausgeglichen werden kann. 
Aus Sicht der einen Zuschlag erhaltenden Landwirte sind spezifische Investitionen dahingehend zu erwarten, dass einerseits Wissen hinsichtlich der Produktion der Umweltleistung in Form von Humankapital erworben und investiert werden muss. Zudem sind spezifische finanzielle Aufwendungen notwendig, welche ausschließlich im Fall einer positiven Vertragserfüllung honoriert werden, denn in der Regel ist keine andere Bewirtschaftung oder Nutzung der vertraglich gebundenen Fläche möglich.

In diesem Zusammenhang zeigt sich zudem die große praktische Relevanz der fundamentalen Transformation insbesondere bei der Frage der Ausgestaltung der Zuschlagserteilung im Rahmen einer wiederholten Ausschreibung.

Bezüglich der Ausprägung der Dimension Häufigkeit ist bedingt durch die innerhalb von Agrarumweltprogrammen in der Regel vorgeschriebene Vertragslaufzeit von fünf Jahren nicht zu erwarten, dass eine identische Transaktionsbeziehung im Rahmen einer sich anschließenden Ausschreibung erneut zustande kommen wird. Demzufolge sind die hier relevanten Transaktionen als einmalig oder in seltenen Fällen als gelegentlich einzuschätzen. Dies besagt jedoch nicht, dass sich die Transaktionspartner nicht regelmäßig und über lange Zeiträume hinweg in einer Transaktionsbeziehung befinden und demzufolge sowohl ein Reputationsaufbau als auch die Minderung von Transaktionskosten ermöglicht werden kann.

Abschließend und unter Einbeziehung der in diesem Kapitel erfolgten Argumentation hat sich gezeigt, dass die als einseitige Marktkontrolle bezeichnete Ausgestaltung einer Beherrschungsstruktur für spezifische Transaktionsbeziehungen zwischen einem staatlichen Akteur auf der Nachfrage- und einer Vielzahl von Unternehmern auf der Angebotsseite als notwendige Erweiterung des Analyserahmens anzusehen ist. 


\section{Die Umsetzung und Ergebnisse der Ausschreibungen}

Mit dem Ziel, das in Abschnitt 5.2.5 abgeleitete und hier zur Honorierung von Umweltleistungen als bestmöglich eingeschätzte Ausschreibungsdesign auf seine praktische Tragfähigkeit sowie Fragen der Preiskalkulation und des Bieterverhaltens hin zu untersuchen, wurde im Frühjahr 2004 mit der Umsetzung des Honorierungskonzepts im Landkreis Northeim begonnen.

Insgesamt wurden in der Modellregion zwei Ausschreibungsverfahren durchgeführt, wobei die Ausschreibung 2004/2005 nachfolgend in Abschnitt 7.1 und die Ausschreibung 2006 in Abschnitt 7.2 thematisiert wird.

Dabei werden für beide Ausschreibungsrunden zu Beginn grundsätzliche Aspekte der Umsetzung angesprochen und im Anschluss die Ausschreibungsergebnisse jeweils separat für die ökologischen Güter Grünland I, II und III sowie zusammenfassend diskutiert. Abschließend erfolgt auf der Grundlage von schriftlichen Befragungen die Einbeziehung der Preiskalkulation und des Bieterverhaltens der Landwirte.

Eine zusammenfassende Gegenüberstellung sowohl der Ausschreibungs- als auch der Befragungsergebnisse wird für beide Ausschreibungsverfahren in Abschnitt 7.3 vorgenommen.

\subsection{Die Ausschreibung 2004/2005}

Die erste Ausschreibung wurde im Zeitraum 2004/2005 durchgeführt und im weiteren Verlauf werden in Abschnitt 7.1.1 einführend das grundsätzliche Vorgehen mit dem Schwerpunkt auf dem zeitlichen Ablauf und der Beiratsentscheidung vorgestellt. Den maßgeblichen Aspekt der Untersuchung stellen die sich in der praktischen Umsetzung herausgebildeten Angebotspreise dar, welche in den Abschnitten 7.1.2, 7.1.3 und 7.1.4 jeweils für die ökologischen Güter Grünland I, II und III und in Abschnitt 7.1.5 zusammenfassend betrachtet werden. ${ }^{122}$

Des Weiteren wird in Abschnitt 7.1.6 die Preiskalkulation und das Bieterverhalten der Landwirte auf der Grundlage einer parallel zum Ausschreibungsverfahren durchgeführten schriftlichen Befragung untersucht.

\footnotetext{
${ }^{122}$ Fragen der Vor-Ort-Kontrolle der ökologischen Güter entsprechend der im Honorierungskonzept implementierten Vorgehensweise werden hier nicht angesprochen.
} 


\subsubsection{Grundlagen, zeitlicher Ablauf und Beiratsentscheidung}

Zu Beginn der Umsetzung erfolgte im Frühjahr 2004 in Zusammenarbeit aller Projektmitarbeiter die Erstellung der Ausschreibungsunterlagen. Diese wurden in Anlehnung an bereits bestehende Antragsformulare gestaltet, um so eine an der Verwaltungspraxis orientierte Erprobung zu ermöglichen und die Landwirte neben dem für sie bislang unbekannten Instrument eines Ausschreibungsverfahrens nicht mit grundlegend neuartigen Unterlagen zu konfrontieren. ${ }^{123}$

Den Ausgangspunkt der praktischen Umsetzung markierte die Sitzung des Regionalen Beirats Northeim am 19. April 2004, im Rahmen derer einstimmig beschlossen wurde, die ökologischen Güter Grünland I, II und III nachzufragen. ${ }^{124}$ Hinsichtlich der Verteilung des für die Ausschreibung insgesamt zur Verfügung stehenden Budgets von $30.000 €^{125}$ auf die drei Qualitätsstufen ökologischer Güter wurde seitens des Beirats entschieden $20.000 €$ für Grünland I, $5.000 €$ für Grünland II und $5.000 €$ für Grünland III zu verwenden. Je nach Aufteilung der Angebote auf die einzelnen ökologischen Güter wurde zudem die Option eingeräumt, bei einem großen Anteil ökologisch hochwertiger Angebote im Rahmen der Zuschlagserteilung eine Verschiebung der Teilbudgets von Grünland I hin zu Grünland II und speziell hin zu Grünland III vorzunehmen.

Entsprechend des Nachfragekatalogs wurde die Ausschreibung am 01. Juni 2004 mit Anzeigen in regionalen Tageszeitungen, der Zeitschrift „Land und Forst“ und über ein Rundschreiben des Amts für Agrarstruktur Göttingen öffentlich bekannt gemacht. Zur Vorstellung des Ausschreibungsverfahrens und zur Information der Landwirte wurden im weiteren Verlauf drei Informationsveranstaltungen im Landkreis Northeim durchgeführt, während derer Fragen von Landwirten beantwortet und die Ausschreibungsunterlagen bereitgestellt wurden.

\footnotetext{
${ }^{123}$ Die vollständigen Ausschreibungsunterlagen befinden sich im Anhang 1 dieser Arbeit.

${ }^{124}$ Die jeweiligen Anforderungen an die ökologischen Güter lassen sich an dieser Stelle wie folgt zusammenfassen: Für Grünland I das Vorkommen von mindestens acht Kräuterarten in jeder Kontrollparzelle; für Grünland II das Vorkommen von mindestens acht Kräuterarten in jeder Kontrollparzelle, wobei davon oder zusätzlich dazu mindestens zwei Arten Zielarten sein müssen; für Grünland III das Vorkommen von mindestens acht Kräuterarten, wobei davon oder zusätzlich dazu mindestens vier Arten Zielarten sein müssen. Eine Auflistung aller Zielarten findet sich als Teil der Ausschreibungsunterlagen in Anhang 1.

${ }^{125}$ Das Budget wurde von der Umweltlotterie „BINGO!“ zur Verfügung gestellt.
} 
Teilnahmeberechtigt waren an der Ausschreibungsrunde unabhängig von ihrer Rechtsform alle konventionell und ökologisch wirtschaftende Betriebe im Hauptund Nebenerwerb mit ihren im Landkreis Northeim bewirtschafteten Flächen. Zudem waren die Bieter verpflichtet, ihren landwirtschaftlichen Betrieb und die angebotenen Grünlandflächen für die Zeit der Vertragsdauer selbst zu bewirtschaften.

Die Angebotsabgabe erfolgte schlagbezogen und jeder Landwirt konnte für alle ökologischen Güter mehrere Schläge anbieten, wobei für jeden dieser Schläge ein individuelles Angebot abgegeben werden musste. ${ }^{126}$ Demzufolge bestand die Möglichkeit, sich bei der Wahl der ökologischen Güter und der Kalkulation der Angebotspreise an den spezifischen Produktionsbedingungen $\mathrm{zu}$ orientieren und differenzierte Angebote abzugeben. Durch die a priori unbekannte Anzahl von Anbietern und einem Budget, dessen Begrenztheit aber nicht dessen Höhe den Landwirten zum Zeitpunkt der Angebotsabgabe bekannt war, wurde die für das Gelingen einer Ausschreibung notwendige Konkurrenzsituation sichergestellt.

Die Angebotsfrist endete nach sechs Wochen am 10. Juli 2004 und innerhalb von einer Woche wurden alle fristgerecht eingegangenen und formal korrekten Angebote seitens der Projektmitarbeiter bewertet.

Der Angebotsbewertung zugrunde gelegt waren als ökologisches Zuschlagskriterium die Einstufung der ökologischen Güter entsprechend ihres ökologischen Nutzens in die Güterklassen Grünland I, Grünland II und Grünland III, um dann innerhalb dieser Abstufungen anhand des ökonomischen Zuschlagskriteriums - dem Angebotspreis pro Hektar - bis zur Ausschöpfung der zur Verfügung stehenden Teilbudgets die jeweils wirtschaftlichsten Angebote auswählen zu können. Dabei konnte bedingt durch die schlagbezogene Angebotsabgabe mit einem einzelnen Landwirt je nach angebotenem ökologischen Gut und Preis pro Hektar für alle, keinen oder einige Schläge ein Vertrag geschlossen werden.

Bedingt durch die Einbindung der Ausschreibung in ein Forschungsprojekt und sich daraus ergebende Restriktionen betrug die Vertragslaufzeit ein Jahr und nicht die in Agrarumweltprogrammen vorgeschriebenen mindestens fünf Jahre. Aus ökologischer Sicht ist hier anzumerken, dass eine grundlegende Veränderung der

\footnotetext{
${ }^{126}$ Die Mindestgröße der angebotenen Fläche wurde auf 0,25 Hektar festgelegt.
} 
Vegetationsstruktur einen längeren Zeitraum als ein Jahr erforderlich machen würde und in dieser Ausschreibung vorwiegend der Erhalt bestehender Artenvielfalt honoriert wurde. Diese Kritik ist jedoch dahingehend zu relativieren, dass zumindest finanzielle Anreize für den Erhalt bestehender Artenvielfalt geschaffen wurden und nicht zuletzt ganz pragmatisch festzuhalten ist, dass eine anderweitige Ausgestaltung nicht möglich gewesen wäre, weshalb im Folgenden der Fokus auf der Angebotsabgabe und den Angebotspreisen liegt.

Die Kontrolle der ökologischen Güter erfolgte anhand definierter Kontrollparzellen bis zum 31. Juli 2005. Eine Kontrollparzelle umfasst die Größe von 12,6 m², was einem Radius von 2 m entspricht, und mit einer zunehmenden Flächengröße ist eine höhere Anzahl von Kontrollparzellen vorgesehen, um die Zielerreichung auf der gesamten Fläche sicherzustellen. ${ }^{127}$ Eine Auszahlung an die Landwirte wurde nach einer erfolgreichen Kontrolle im August 2005 vorgenommen, wobei innerhalb dieses ersten Ausschreibungsverfahrens eine Fehlertoleranz von 25 \% eingeräumt wurde. Für den Fall, dass die angebotene pflanzliche Artenvielfalt nicht erreicht wurde, erfolgte - darüber hinaus, dass der Landwirt keine Auszahlung erhielt - keinerlei Sanktion.

\subsubsection{Ergebnisse - Ökologisches Gut Grünland I}

Bevor im weiteren Verlauf auf die Angebotspreise für die ökologischen Güter Grünland I eingegangen wird, erscheint es notwendig, vorab eine Beschreibung der damit verbundenen Anforderungen an die pflanzliche Biodiversität zu geben. Für das Erreichen des ökologischen Guts Grünland I ist die Mindestvoraussetzung das Vorhandensein von acht verschiedenen Kräuterarten pro Kontrollparzelle, was innerhalb des Forschungsprojekts die relativ geringste Anforderung darstellt und somit auch den relativ geringsten ökologischen Nutzen erwarten lässt.

Zur Analyse der Angebotspreise werden eingangs alle formal korrekten Angebote betrachtet, aus denen im Rahmen der Zuschlagserteilung die Auswahl zu treffen war. Auf die angenommenen Angebote wird anschließend eingegangen, bevor sich der

\footnotetext{
${ }^{127}$ Für eine detaillierte Darstellung des Konzepts der Kontrollparzelle und weiterführende Aspekte der Kontrolle ökologischer Güter siehe Bertke (2005).
} 
Blick auf die Ergebnisse der Kontrolle richtet, wobei diesbezüglich ausschließlich die Angebotspreise und die Auszahlungen an die Landwirte betrachtet werden.

\subsubsection{Eingegangene Angebote}

Insgesamt wurden für das ökologische Gut Grünland I 146 Schläge angeboten, von denen 16 formale Mängel ${ }^{128}$ aufgewiesen haben oder nicht fristgerecht eingegangen sind, so dass 130 Schläge von 27 Landwirten zur Bewertung standen. Diese Angebote werden im Folgenden als die eingegangenen Angebote bezeichnet und umfassen eine Gesamtfläche von 221,16 ha.

Von zentralem Interesse sind nun die Angebotspreise, da sie die Grundlage für einen effizienten Mitteleinsatz darstellen. Die Angebotspreise pro Hektar für das in seinem ökologischen Nutzen relativ geringwertigste ökologische Gut Grünland I umfassen eine Spanne von $40 €$ bis $250 €$, die mit einem Mittelwert von 100,92 €, einem Median von $100 €$ und einer Standardabweichung von 47,18 € die in einem hohen Maß unterschiedlichen Angebote verdeutlicht. Dies veranschaulicht zudem Abbildung 7-1, in der die einzelnen Angebotspreise pro Hektar und die jeweilige Häufigkeit ihres Auftretens dargestellt sind.

\footnotetext{
${ }^{128}$ Beispielsweise eine zu geringe Flächengröße oder fehlende Preisangaben.
} 


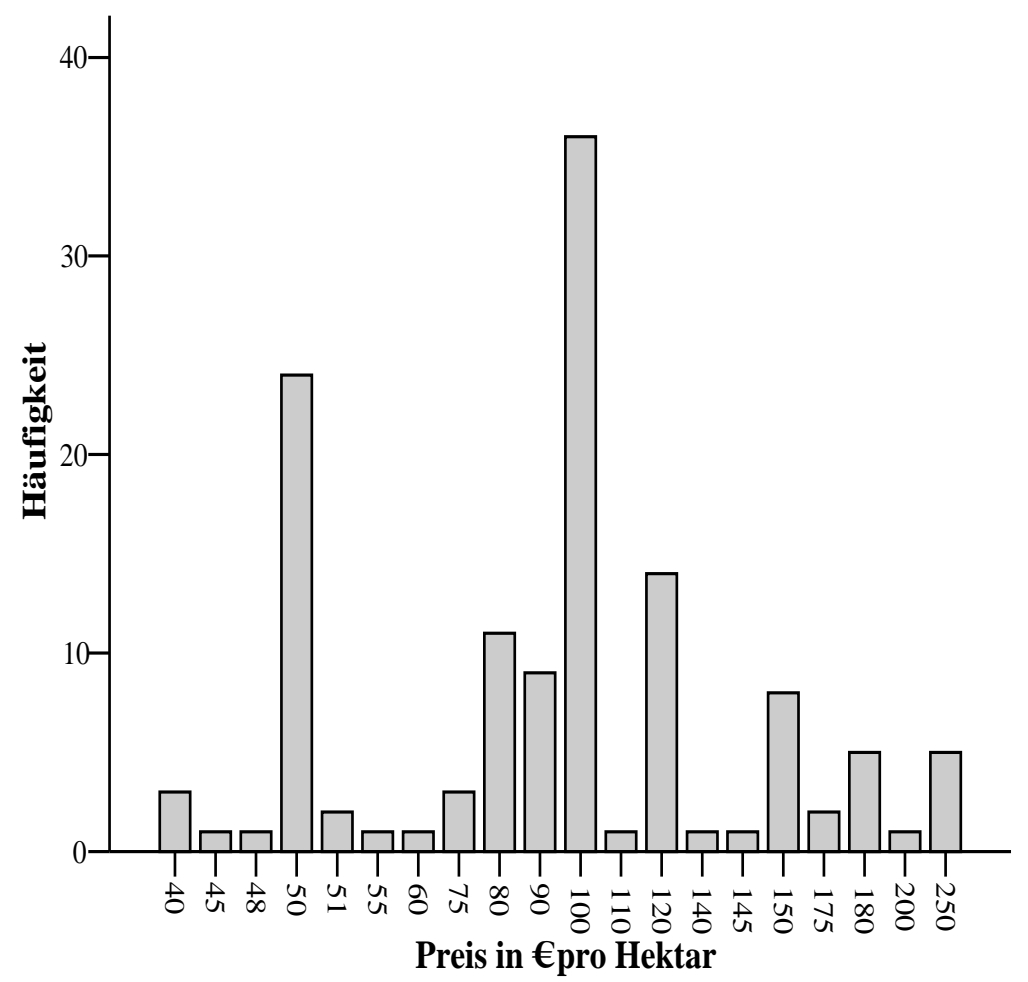

Abb. 7-1: Angebotspreise in $€$ pro Hektar der eingegangenen Angebote für das ökologische Gut Grünland I und Häufigkeit ihres Auftretens (2004/2005)

Quelle: Eigene Darstellung nach eigenen Berechnungen.

Die individuellen Angebotspreise zeigen bei $50 €$ und bei $100 €$ zwei maßgebliche Häufungen, doch nichtsdestoweniger verdeutlicht die insgesamt vorhandene Unterschiedlichkeit und große Anzahl der Angebote das praktische Potential, im Rahmen der Zuschlagserteilung eine umfassende Vertragsdifferenzierung vornehmen zu können. Einen Überblick über die Ergebnisse der eingegangenen Angebote für das ökologische Gut Grünland I gibt Tabelle 7-1.

Tab. 7-1: Ergebnisse der eingegangenen Angebote für das ökologische Gut Grünland I (2004/2005)

\begin{tabular}{|l|c|c|c|c|}
\hline $\mathrm{n}=130$ & Preisspanne & Mittelwert & Median & Std.abw. \\
\hline Grünland I & $40-250 € /$ ha & $100,92 € /$ ha & $100,00 € /$ ha & $47,18 € /$ ha \\
\hline
\end{tabular}

Quelle: Eigene Berechnungen.

Folglich spiegeln sich die differenzierten Angebotspreise auch in der Summe je Schlag wider, die sich aus der Flächengröße multipliziert mit dem Angebotspreis pro Hektar ergibt. Im Minimum beträgt sie 12,95 € und erstreckt sich mit einem 
Mittelwert von 156,81 $€$ und einem Median von $116,47 €$ bis hin zu einem maximalen Wert von 613,98 €. Die Gesamtsumme aller eingegangenen Angebote beträgt 20.385,57 $€$ und liegt gering über dem seitens des Regionalen Beirats Northeim beschlossenen Teilbudgets.

\subsubsection{Angenommene Angebote}

Aufgrund des begrenzten Budgets und der Option, bei vielen ökologisch hochwertigen Angeboten von der Beiratsentscheidung abzuweichen und weniger als $20.000 €$ für das ökologische Gut Grünland I einzusetzen, konnte nicht allen eingegangenen Angeboten ein Zuschlag erteilt werden.

Von den 130 angebotenen Schlägen wurden 109 Schläge von 20 Landwirten angenommen, was einer Fläche von 198,25 ha und einem Budget von 16.100,84 € entspricht. Die Summen je Schlag umfassen mit dem Mittelwert von 147,71 $€$ und einem Median von 104,50 $€$ einen Bereich von 12,95 $€$ bis 569,97 $€$. Die Angebotspreise der angenommenen Schläge pro Hektar bewegen sich im Bereich von $40 €$ bis $145 €$, was auch unter Berücksichtigung eines Mittelwerts von 84,59 $€$, des Medians von $90 €$ und der Standardabweichung von 26,45 € zeigt, welche große Differenz der Angebotspreise für einen identischen ökologischen Nutzen - trotz der bestehenden Häufungen bei $50 €$ und $100 €$ - weiterhin vorhanden ist.

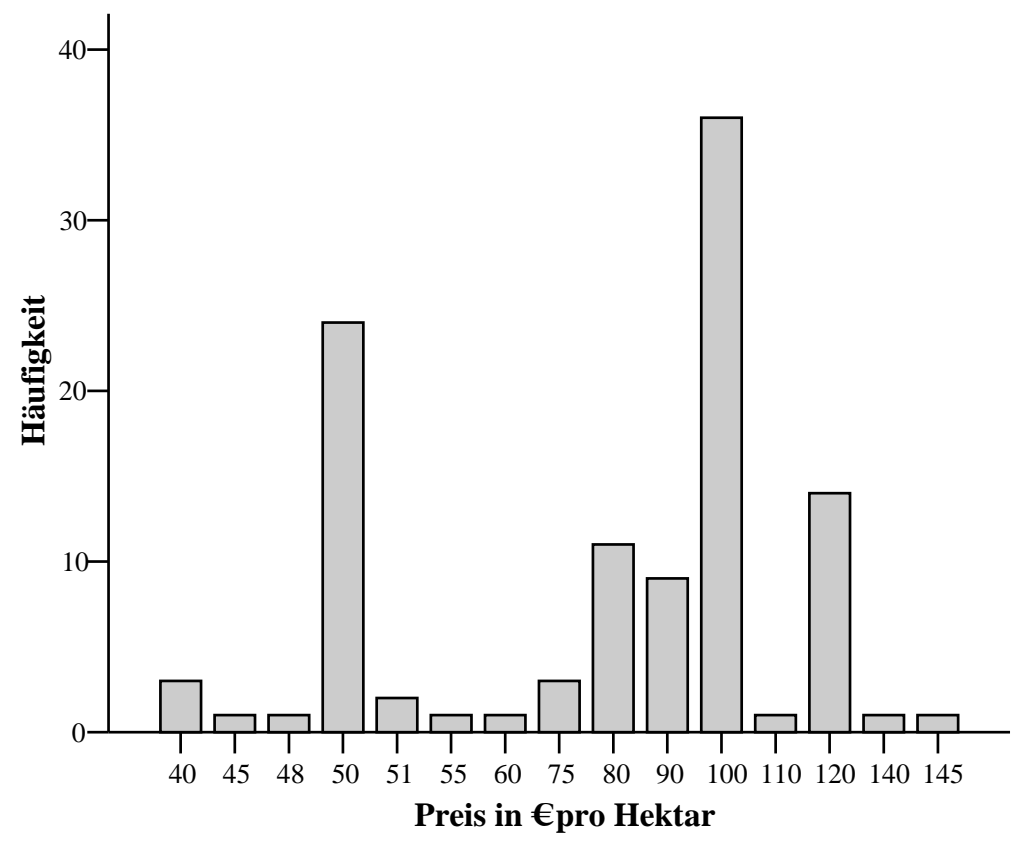

Abb. 7-2: Angebotspreise in $€$ pro Hektar der angenommenen Angebote für das ökologische Gut Grünland I und Häufigkeit ihres Auftretens (2004/2005)

Quelle: Eigene Darstellung nach eigenen Berechnungen. 
Infolgedessen konnte bei der Zuschlagserteilung eine Vertragsdifferenzierung unter Berücksichtigung der in Abbildung 7-2 veranschaulichten individuellen Preiskalkulation erfolgen. Die Ergebnisse der angenommenen Angebote für das ökologische Gut Grünland I fasst Tabelle 7-2 zusammen.

Tab. 7-2: Ergebnisse der angenommenen Angebote für das ökologische Gut Grünland I (2004/2005)

\begin{tabular}{|l|c|c|c|c|}
\hline $\mathrm{n}=109$ & Preisspanne & Mittelwert & Median & Std.abw. \\
\hline Grünland I & $40-145 € /$ ha & $84,59 € /$ ha & $90,00 € /$ ha & $26,45 € /$ ha \\
\hline
\end{tabular}
Quelle: Eigene Berechnungen.

Neben den angesprochenen Angebotspreisen stellt sich ferner die Frage nach einem praktischen Institutionenvergleich zweier Honorierungsinstrumente, um Aussagen über die Effizienzvorteile einer Ausschreibung im Vergleich zu einer einheitlichen Prämienzahlung treffen zu können.

Da bedingt durch die spezifische Ausgestaltung des Honorierungskonzepts, mit der darin verfolgten ergebnisorientierten Honorierung ökologischer Leistungen auf Grünlandflächen, zum Zeitpunkt der Umsetzung in der Modellregion kein Agrarumweltprogramm mit einer einheitlichen Prämienzahlung als Vergleichsmaßstab existierte, wird nachfolgend auf die innerhalb der „Richtlinie über die Gewährung von Zuwendungen für Niedersächsische Agrarumweltprogramme (NAU) 2005“ relevante Fördermaßnahme B „Förderung extensiver Grünlandnutzung“ und die dort angebotene jährliche Prämienzahlung in Höhe von $103 €$ pro Hektar zurückgegriffen.

Die ökologischen Anforderungen zum Erhalt der Prämienzahlung liegen hierbei unter dem im Rahmen des Ausschreibungsverfahrens durch das ökologische Gut Grünland I repräsentierten ökologischen Nutzen, da lediglich eine extensive Nutzung von Grünlandflächen ohne zusätzliche Nachweise an die pflanzliche Artenvielfalt erforderlich ist.

Ein Vergleich dieser Einheitsprämie von $103 €$ pro Hektar mit den Angebotspreisen der angenommenen Angebote zeigt, dass sowohl der Mittelwert in Höhe von 84,59 € pro Hektar als auch der Median in Höhe von $90 €$ pro Hektar deutlich unter der einheitlichen Prämie liegen.

Demzufolge kann die Vorteilhaftigkeit eines Ausschreibungsverfahrens gegenüber einer einheitlichen Prämienzahlung anhand von zwei Beispielrechnungen aufgezeigt 
werden. Um die innerhalb der Ausschreibung unter Vertrag genommene Fläche von 198,25 ha mit der Hektarprämie von $103 €$ zu erreichen, hätten 20.419,75 € aufgewendet werden müssen. Demgegenüber konnte diese Fläche innerhalb des Ausschreibungsverfahrens für 16.100,84 € nachgefragt werden, was einer Ersparnis von 4.318,91 € oder auch 21,2 \% entspricht. Infolgedessen hätte je nach unterstellter Budgetrestriktion mit dem hier eingesetzten Budget von 16.100,84 $€$ bei einer Einheitsprämie von $103 €$ pro Hektar lediglich eine Fläche von 156,32 ha und nicht die im Zuge der Ausschreibung realisierte Grünlandfläche von 198,25 ha angenommen werden können.

\subsubsection{Auszahlung nach der Kontrolle}

Nachdem der Zeitpunkt bis zur Zuschlagserteilung betrachtet ist, stellt sich die Frage, welche Verträge letztlich von den Landwirten erfüllt wurden.

Von den 109 angenommenen konnte auf 87 Schlägen die angebotene pflanzliche Artenvielfalt nachgewiesen werden, ${ }^{129}$ wobei diese Gesamtfläche 156,02 ha umfasst. Die Ausfallquote - also der Anteil der Angebote, bei denen die ökologische Leistung nicht angebots- bzw. vertragskonform erbracht wurde - liegt bei 20,2 \% der angenommenen Schläge. Die in Abbildung 7-3 dargestellten Angebotspreise pro Hektar der positiv kontrollierten Flächen weisen einen Mittelwert von 79,94 €, einen Median von $80 €$ und eine Standardabweichung von 26,68 $€$ auf und differieren von $40 €$ bis $140 €$.

\footnotetext{
129 Für einen Überblick über die Kontrolle der ökologischen Güter innerhalb der Ausschreibung 2004/2005 siehe Bertke et al. (2005).
} 


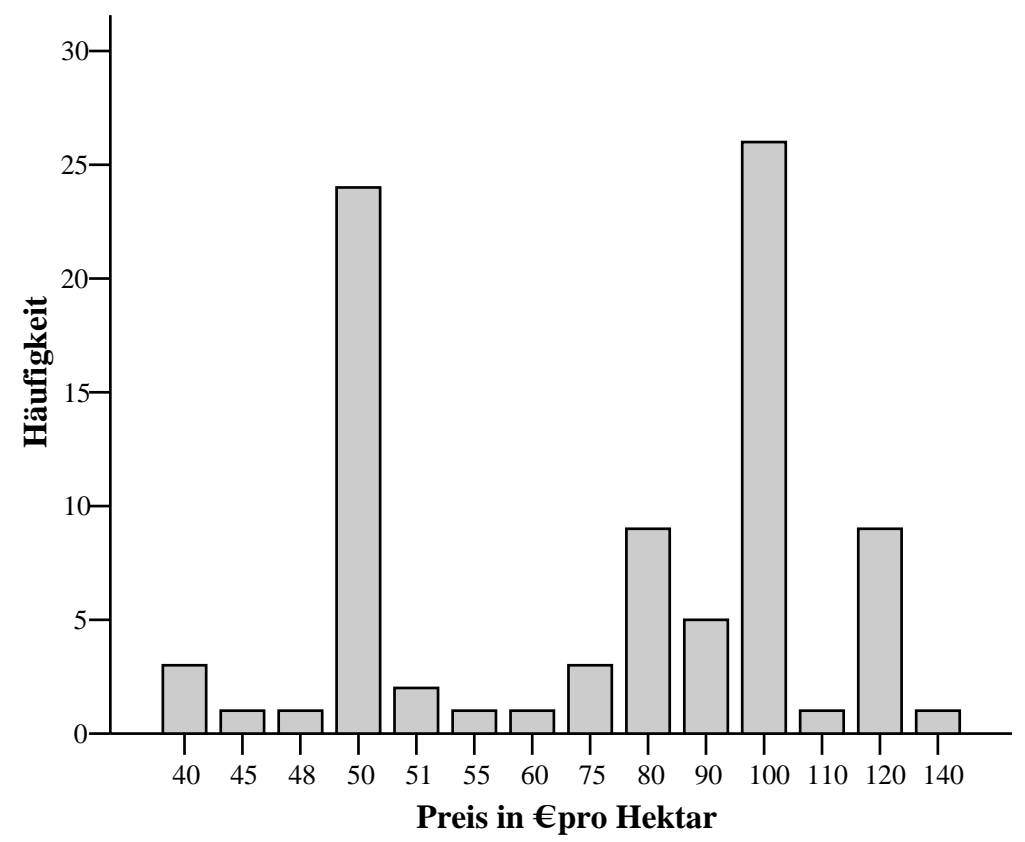

Abb. 7-3: Angebotspreise in $€$ pro Hektar der erfüllten Angebote für das ökologische Gut Grünland I und Häufigkeit ihres Auftretens (2004/2005)

Quelle: Eigene Darstellung nach eigenen Berechnungen.

Die Preise zeigen, dass auch zu den relativ geringen Kosten die angebotene pflanzliche Artenvielfalt erbracht wurde und derartige Unterschiede durch ein Ausschreibungsverfahren sowohl aufgedeckt als auch genutzt werden konnten. Tabelle 7-3 gibt einen Überblick über die wesentlichen Ergebnisse der erfüllten Angebote.

Tab. 7-3: Ergebnisse der erfüllten Angebote für das ökologische Gut Grünland I $(2004 / 2005)$

\begin{tabular}{|l|c|c|c|c|}
\hline $\mathrm{n}=87$ & Preisspanne & Mittelwert & Median & Std.abw. \\
\hline Grünland I & $40-140 € /$ ha & $79,94 € /$ ha & $80,00 € /$ ha & $26,68 € /$ ha \\
\hline
\end{tabular}

Quelle: Eigene Berechnungen.

Demzufolge wurden insgesamt $11.852,90 €$ an die Landwirte gezahlt, wobei die Auszahlungssummen pro Schlag eine Spanne von $13 €$ bis $570 €$ umfassen, die durch eine Standardabweichung von 120,65 € gekennzeichnet ist. Der entsprechende Mittelwert beträgt 136,24 € und der Median liegt bei 92,57 €.

Zur Beurteilung der ökonomischen Vorteilhaftigkeit eines Ausschreibungsverfahrens wird an dieser Stelle erneut die oben bereits angesprochene Prämienzahlung für eine 
Förderung extensiver Grünlandnutzung aus den Niedersächsischen Agrarumweltprogrammen aufgegriffen.

Ebenfalls sind für die Hektarpreise der durch die Landwirte erfüllten Angebote und ihrem Vergleich mit der in Niedersachsen für eine extensive Grünlandnutzung angebotenen Prämienzahlung Effizienzvorteile eines Ausschreibungsverfahrens zu erkennen.

Für die innerhalb der Ausschreibung auf 156,02 ha Grünlandfläche erbrachte Umweltleistung mussten insgesamt 11.852,90 $€$ aufgewendet werden. Demgegenüber hätte bei einer einheitlichen Prämienzahlung von $103 €$ pro Hektar hierfür eine Gesamtsumme von 16.070,06 € eingesetzt werden müssen, was einer durch die Ausschreibung realisierten Einsparung von 4.217,16 $€$ oder 26,2 \% entspricht. Dem gleichbedeutend ist, dass bei einer Honorierung entsprechend der Einheitsprämie von $103 €$ pro Hektar lediglich eine 115,08 ha umfassende und nicht die mit der Ausschreibung erreichte Grünlandfläche von 156,02 ha hätte realisiert werden können.

\subsubsection{Ergebnisse - Ökologisches Gut Grünland II}

Für das Erreichen des ökologischen Guts Grünland II ist die Mindestvoraussetzung das Vorhandensein von acht verschiedenen Kräuterarten pro Kontrollparzelle, wobei von diesen acht oder zusätzlich dazu zwei Arten Bestandteil eines Zielartenkatalogs sein müssen. Somit gehen die spezifischen Anforderungen qualitativ über die für Grünland I hinaus und lassen einen höheren ökologischen Nutzen erwarten.

Zur Analyse der Angebotspreise werden eingangs alle fristgerecht eingegangenen und formal korrekten Angebote betrachtet, aus denen im Rahmen der Zuschlagserteilung die Auswahl zu treffen war. Da alle Angebote einen Zuschlag erhalten haben, werden die angenommenen Angebote hier unmittelbar ebenfalls betrachtet, bevor sich der Blick abschließend auf die Ergebnisse der Kontrolle der ökologischen Güter richtet.

\subsubsection{Eingegangene und angenommene Angebote}

Für die ökologischen Güter Grünland II wurden von 18 Landwirten insgesamt 35 Schläge angeboten, von denen drei Angebote formale Mängel aufwiesen, so dass letztlich 32 Schläge von 16 Landwirten zur Entscheidung standen. Diesen 32 
Schlägen konnte ausnahmslos ein Zuschlag erteilt werden und die angenommene Gesamtfläche beträgt 53,33 Hektar.

Mit einem Mittelwert von 141,75 €, dem Median $140 €$ und der Standardabweichung 59,55 € zeigen sich ebenfalls für die ökologischen Güter Grünland II in der Praxis sehr unterschiedliche Angebotspreise pro Hektar, welche eine Spanne von $55 €$ bis $300 €$ umfassen. Somit sind auch für die einen mittleren ökologischen Nutzen repräsentierenden ökologischen Güter in der Modellregion unterschiedliche Kosten der landwirtschaftlichen Betriebe zu erwarten, die durch ein Ausschreibungsverfahren aufgedeckt werden konnten und eine Vertragsdifferenzierung für eine identische Umweltleistung ermöglicht haben. Die eingegangenen und angenommenen Angebote sind in Abbildung 7-4 jeweils mit den Angebotspreisen pro Hektar und der Häufigkeit ihres Auftretens dargestellt.

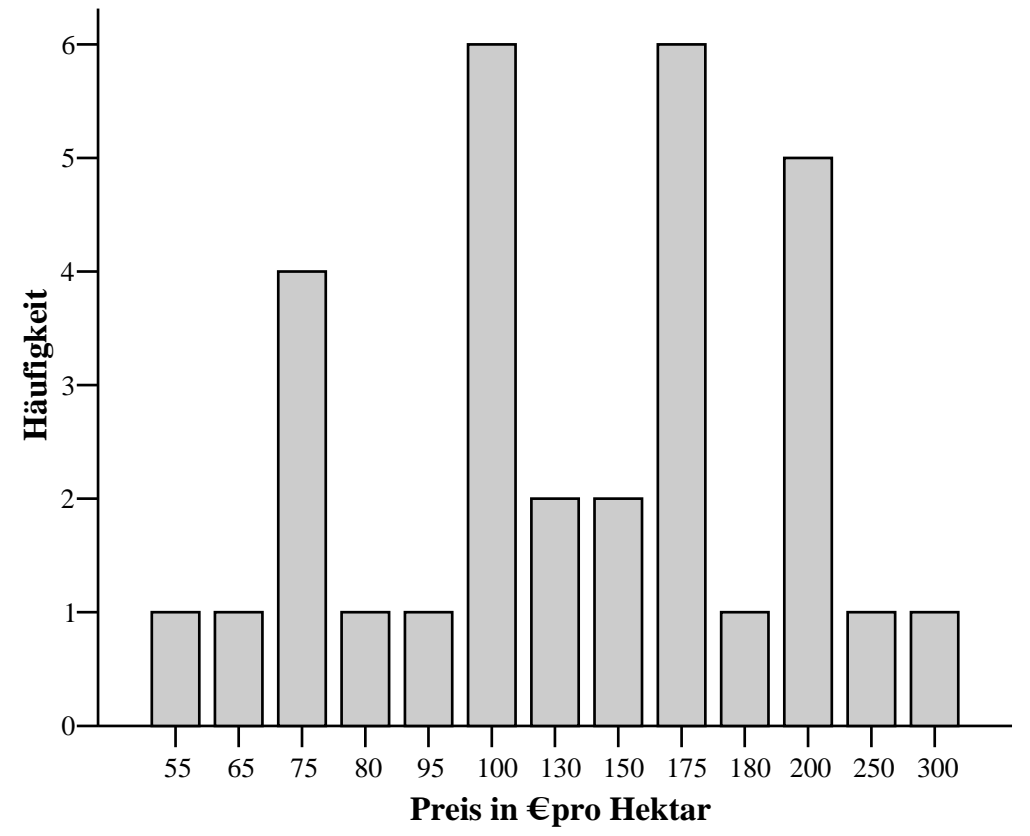

Abb. 7-4: Angebotspreise in $€$ pro Hektar der eingegangenen und angenommenen Angebote für das ökologische Gut Grünland II und Häufigkeit ihres Auftretens (2004/2005)

Quelle: Eigene Darstellung nach eigenen Berechnungen.

Die sich aus der Multiplikation der Flächengröße und des Angebotspreises pro Hektar ergebenden Summen je Schlag umfassen Beträge von 19,35 € bis 604,85 € und spiegeln mit einem Mittelwert von 217,94 € und dem Median 182,53 € ebenfalls die großen Unterschiede der unter Vertrag genommenen Schläge wider. Die Gesamtsumme aller angenommenen Angebote beträgt 6.974,03 € und liegt über dem 
innerhalb des Nachfragekatalogs beschlossenen Teilbudgets von $5.000 €$. Durch die eingeräumte Möglichkeit, diese Finanzmittel gegebenenfalls zu Lasten des Budgets für Grünland I zu erhöhen, konnte jedoch eine entsprechende Umschichtung vorgenommen werden. Die hinsichtlich der Angebotspreise zentralen Ergebnisse der eingegangenen und angenommenen Angebote sind für das ökologische Gut Grünland II in Tabelle 7-4 dargestellt.

Tab. 7-4: Ergebnisse der eingegangenen und angenommenen Angebote für das ökologische Gut Grünland II (2004/2005)

\begin{tabular}{|l|c|c|c|c|}
\hline $\mathrm{n}=32$ & Preisspanne & Mittelwert & Median & Std.abw. \\
\hline Grünland II & $55-300 € /$ ha & $141,75 € /$ ha & $140,00 € /$ ha & $59,55 € /$ ha \\
\hline
\end{tabular}

Quelle: Eigene Berechnungen.

Ein praktischer Institutionenvergleich zwischen der durchgeführten Ausschreibung und einer einheitlichen Prämienzahlung, wie er für das ökologische Gut Grünland I durchgeführt werden konnte, ist für das ökologische Gut Grünland II problematischer.

Es kann dennoch näherungsweise die innerhalb der Agrarumweltförderung des Bundes im GAK-Rahmenplan 2005-2008 vorgesehene Prämienzahlung von $130 €$ pro Hektar herangezogen werden. ${ }^{130}$ Hierbei zeigt sich, dass die im Rahmen der Ausschreibung angenommene Grünlandfläche von 53,33 Hektar mit einer Prämienzahlung von $130 €$ für insgesamt 6.932,90 $€$ hätte nachgefragt werden können. Dies entspricht gegenüber des im Rahmen des Ausschreibungsverfahrens eingesetzten Teilbudgets von 6.974,03 € einer minimalen Einsparung, wobei hier durch die Wahl eines geringeren Reservationspreises innerhalb der Ausschreibung hätte Einfluss genommen werden können.

\subsubsection{Auszahlung nach der Kontrolle}

Ein Nachweis der angebotenen Artenvielfalt konnte für die ökologischen Güter Grünland II auf 27 der angenommenen 32 Schläge erfolgen, was einer Ausfallquote von 15,63 \% und einer Gesamtfläche von 45,91 Hektar entspricht. Die Preise pro Hektar, zu denen das Vorkommen der pflanzlichen Artenvielfalt durch die Landwirte realisiert werden konnte, umfassen eine Spanne von $55 €$ als geringstem und $250 €$

\footnotetext{
130 Innerhalb der Agrarumweltförderung des Bundes: umweltfreundliche Anbauverfahren auf dem Grünland - Erhaltung pflanzengenetisch wertvoller Grünlandvegetation.
} 
als höchstem Angebotspreis. Der Mittelwert beträgt 136,67 €, der Median $130 €$ und die Standardabweichung 54,79 €. Abbildung 7-5 zeigt die Angebotspreise der erfüllten Angebote in € pro Hektar und die Häufigkeit ihres Vorkommens.

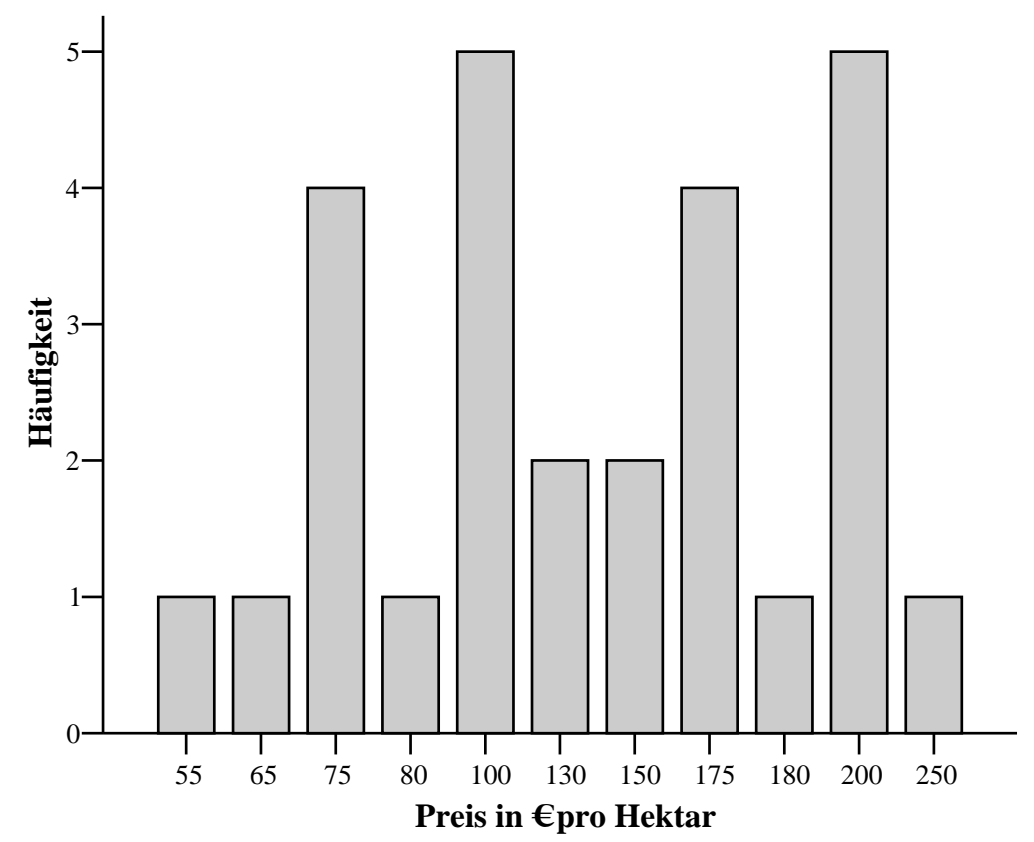

Abb. 7-5: Angebotspreise in $€$ pro Hektar der erfüllten Angebote für das ökologische Gut Grünland II und Häufigkeit ihres Auftretens (2004/2005)

Quelle: Eigene Darstellung nach eigenen Berechnungen.

Somit wurden in der Qualitätsstufe der ökologischen Güter Grünland II insgesamt 5.911,24 $€$ an die Landwirte ausgezahlt, wobei die einzelnen Auszahlungssummen je Schlag eine Spanne von 19,35 € bis 604,85 €, bei einem Mittelwert von 218,94€ und einem Median von 172,73 €, umfassen. Die Ergebnisse der Angebotspreise fasst Tabelle 7-5 zusammen.

Tab. 7-5: Ergebnisse der erfüllten Angebote für das ökologische Gut Grünland II (2004/2005)

\begin{tabular}{|l|c|c|c|c|}
\hline $\mathrm{n}=27$ & Preisspanne & Mittelwert & Median & Std.abw. \\
\hline Grünland II & $55-250 € /$ ha & $136,67 € /$ ha & $130,00 € /$ ha & $54,79 € /$ ha \\
\hline
\end{tabular}

Quelle: Eigene Berechnungen.

Wird auch hier als praktischer Referenzwert die Prämienzahlung innerhalb des GAK-Rahmenplans 2005-2008 in Höhe von $130 €$ pro Hektar für die Erhaltung pflanzengenetisch wertvoller Grünlandvegetation herangezogen, so zeigen sich nun für die von den Landwirten erfüllten Verträge geringe ökonomische Vorteile des 
Ausschreibungsverfahrens. Die auf 45,91 Hektar erbrachten Umweltleistungen haben innerhalb des Forschungsprojekts zu Ausgaben in Höhe von 5.911,24 € geführt, wohingegen auf der Grundlage einer einheitlichen Entlohnung in Höhe von $130 €$ pro Hektar 5.968,30 € hätten eingesetzt werden müssen.

\subsubsection{Ergebnisse - Ökologisches Gut Grünland III}

Den relativ höchsten ökologischen Nutzen lässt die durch das ökologische Gut Grünland III repräsentierte Artenvielfalt erwarten. Die Mindestvoraussetzung ist das Vorhandensein von acht verschiedenen Kräuterarten pro Kontrollparzelle, wobei von diesen acht Kräuterarten oder zusätzlich dazu vier Arten in einem Zielartenkatalog aufgeführt sein müssen.

Im Folgenden wird bei der Analyse der Angebotspreise zuerst der Zeitraum bis zur Zuschlagserteilung betrachtet, um anschließend auf die Auszahlungen an die Landwirte einzugehen.

\subsubsection{Eingegangene und angenommene Angebote}

Insgesamt wurden für das ökologische Gut Grünland III 18 Schläge angeboten, von denen alle fristgerecht und formal korrekt eingegangen sind. Im Rahmen der Zuschlagserteilung konnte ebenfalls allen 18 angebotenen Schlägen von acht Landwirten ein Zuschlag erteilt werden.

Daher werden analog zur Untersuchung des ökologischen Guts Grünland II auch hier die eingegangenen und angenommenen Angebote zusammen betrachtet. Die Gesamtfläche der Angebote beträgt 36,98 ha. Die Preise der angenommenen Angebote pro Hektar zeigen für das ökologische Gut Grünland III im Vergleich zu den oben betrachteten ökologischen Gütern Grünland I und Grünland II mit $250 €$ die geringste Spanne. Jedoch lassen die sich mit einem Mittelwert von 202,78 €, einem Median von $175 €$ und einer Standardabweichung von 78,73 € zwischen $100 €$ und $350 €$ bewegenden Angebotspreise pro Hektar auch für die mit dem relativ höchsten ökologischen Nutzen verbundene pflanzliche Artenvielfalt auf unterschiedliche Produktionskosten schließen. Abbildung 7-6 zeigt die eingegangenen und angenommenen Angebote anhand ihres Angebotspreises pro Hektar und der Häufigkeit ihres Auftretens. 


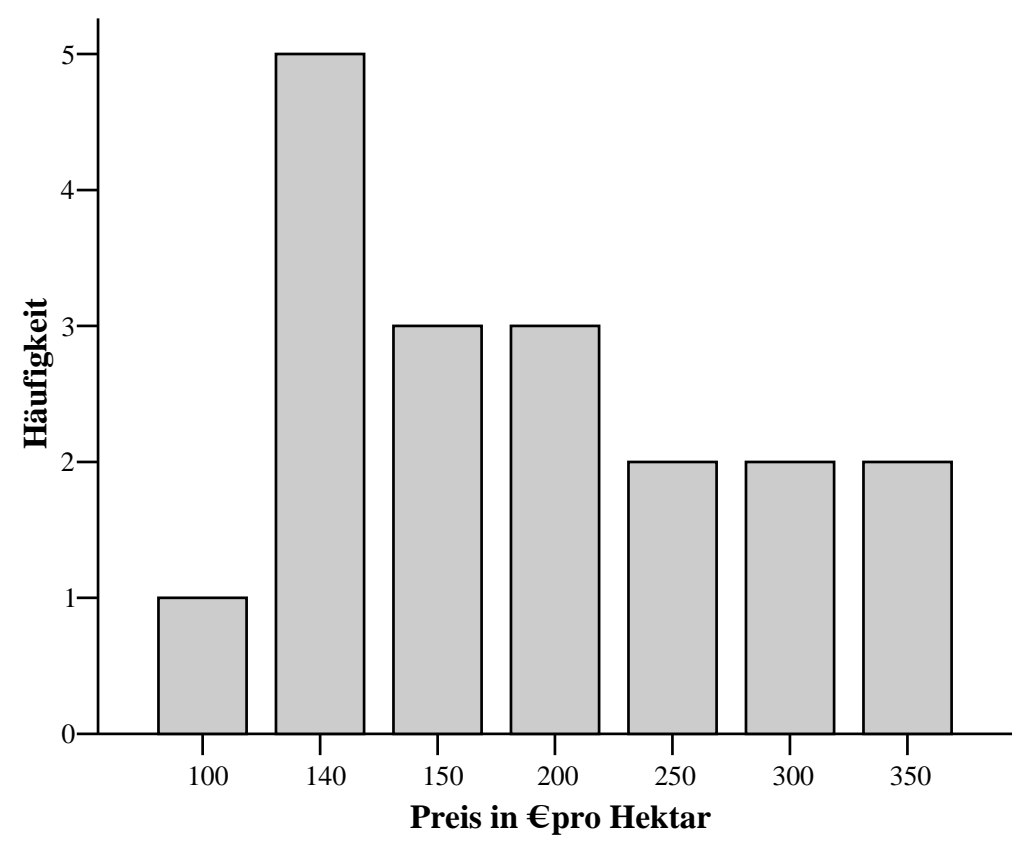

Abb. 7-6: Angebotspreise in $€$ pro Hektar der eingegangenen und angenommenen Angebote für das ökologische Gut Grünland III und Häufigkeit ihres Auftretens (2004/2005)

Quelle: Eigene Darstellung nach eigenen Berechnungen.

Anhand der Summe je angenommenem Angebot lässt sich für die hier betrachtete Artenvielfalt eine sehr große Differenz feststellen, die mit dem Mittelwert 354,91 € und dem Median 142,88 € eine Spanne von $50 €$ bis 1.580,03 $€$ umfasst. Die Gesamtsumme der eingegangenen und angenommenen Angebote beträgt 6.388,31 €. Einen Überblick zu den Angebotspreisen der eingegangenen und angenommenen Angebote für das ökologische Gut Grünland III gibt Tabelle 7-6.

Tab. 7-6: Ergebnisse der eingegangenen und angenommenen Angebote für das ökologische Gut Grünland III (2004/2005)

\begin{tabular}{|l|c|c|c|c|}
\hline $\mathrm{n}=18$ & Preisspanne & Mittelwert & Median & Std.abw. \\
\hline Grünland III & $100-350 € /$ ha & $202,78 € /$ ha & $175,00 € /$ ha & $78,73 € /$ ha \\
\hline
\end{tabular}

Quelle: Eigene Berechnungen.

Ein praktischer Vergleich der durchgeführten Ausschreibung und einer einheitlichen Prämienzahlung, wie er für die ökologischen Güter Grünland I und Grünland II durchgeführt werden konnte, ist für das ökologische Gut Grünland III nicht möglich, da bislang auch näherungsweise noch kein Agrarumweltprogramm existiert, welches 
eine Prämienzahlung und eine Ergebnisorientierung entsprechend der hier honorierten Umweltleistungen beinhaltet.

\subsubsection{Auszahlung nach der Kontrolle}

Die Kontrolle auf den Grünlandflächen hat für das ökologische Gut Grünland III ergeben, dass auf zwölf der angenommenen 18 Schläge die erforderliche Artenvielfalt erreicht und damit der Vertrag erfüllt wurde. Dies entspricht einer Ausfallquote von einem Drittel und einer positiv kontrollierten Gesamtfläche von 25,96 ha.

Bei der Betrachtung der Angebotspreise pro Hektar zeigt sich weiterhin die mit einem Mittelwert von $225 €$, einem Median von $200 €$ und einer Standardabweichung von 83,94 € den Bereich von $100 €$ bis $350 €$ umfassende große Spanne der Angebotspreise. Folglich lässt sich auch für die relativ hochwertigsten ökologischen Güter hinsichtlich der Kosten der landwirtschaftlichen Betriebe auf individuelle Unterschiede schließen, die durch ein Ausschreibungsverfahren in Form der Angebotspreise aufgedeckt und berücksichtigt werden konnten. Die Angebotspreise der erfüllten Angebote in $€$ pro Hektar und die Häufigkeit ihres Vorkommens zeigt Abbildung 7-7.

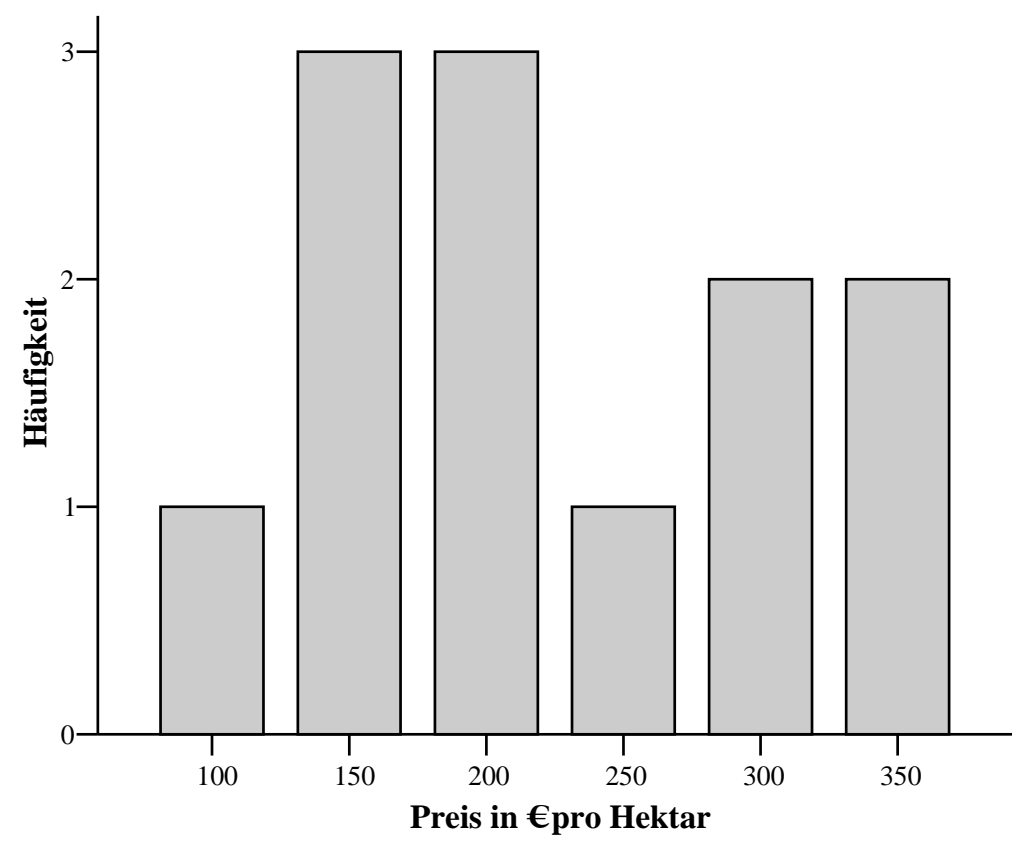

Abb. 7-7: Angebotspreise in $€$ pro Hektar der erfüllten Angebote für das ökologische Gut Grünland III und Häufigkeit ihres Auftretens (2004/2005)

Quelle: Eigene Darstellung nach eigenen Berechnungen. 
Letztlich wurden innerhalb der ökologischen Güter Grünland III 4.781,66 € ausgezahlt. Die Auszahlungssummen je Schlag variieren mit einem Mittelwert von 398,47 €, einem Median von 226,61 € und einer Standardabweichung von 442,19 € in einem Bereich von $50 €$ bis 1.580,03 $€$. Tabelle 7-7 gibt einen zusammenfassenden Überblick über die Preise der innerhalb der Qualitätsstufe Grünland III seitens der Landwirte erfüllten Angebote.

\section{Tab. 7-7: Ergebnisse der erfüllten Angebote für das ökologische Gut Grünland} III (2004/2005)

\begin{tabular}{|l|c|c|c|c|}
\hline $\mathrm{n}=12$ & Preisspanne & Mittelwert & Median & Std.abw. \\
\hline Grünland I & $100-350 € /$ ha & $225,00 € /$ ha & $200,00 € /$ ha & $83,94 € /$ ha \\
\hline
\end{tabular}

Quelle: Eigene Berechnungen.

Wie bei der Betrachtung der eingegangenen und angenommenen Angebote bereits angeführt wurde, besteht für die erfüllten Verträge derzeit ebenfalls keine Vergleichsmöglichkeit mit einer einheitlichen Prämienzahlung.

\subsubsection{Zusammenfassende Ergebnisse der Angebotspreise}

Mit dem Ziel eines direkten Vergleichs der sich innerhalb der drei Abstufungen der ökologischen Güter Grünland herausgebildeten Angebotspreise erfolgt jeweils für die eingegangenen, die angenommenen und die erfüllten Angebote eine zusammenfassende Gegenüberstellung.

\subsubsection{Eingegangene Angebote}

Die Ausschreibungsunterlagen wurden insgesamt von 140 Landwirten angefordert und ein fristgerechtes und formal korrektes Angebot haben 38 Landwirte mit insgesamt 199 Schlägen abgegeben, was einer Gesamtfläche von 350,71 ha entspricht.

Tabelle 7-8 gibt eine Gegenüberstellung der Angebotspreise für alle ökologischen Güter und durch die Preisspannen und Standardabweichungen wird deutlich, dass es jeweils zu sehr differenzierten Angebotspreisen gekommen ist. Darüber hinaus zeigt sich gleichermaßen, dass das Preisniveau erwartungsgemäß mit einem zunehmenden ökologischen Nutzen von Gut Grünland I über Gut Grünland II bis hin zu Gut Grünland III ebenfalls angestiegen ist. 
Tab. 7-8: Ergebnisse der eingegangenen Angebote für die ökologischen Güter Grünland I, II und III (2004/2005)

\begin{tabular}{|l|c|c|c|c|}
\hline & Preisspanne & Mittelwert & Median & Std.abw. \\
\hline Grünland I & $40-250 € /$ ha & $100,92 € /$ ha & $100,00 € /$ ha & $47,18 € /$ ha \\
\hline Grünland II & $55-300 € /$ ha & $141,75 € /$ ha & $140,00 € /$ ha & $59,55 € /$ ha \\
\hline Grünland III & $100-350 € /$ ha & $202,78 € /$ ha & $175,00 € /$ ha & $78,73 € /$ ha \\
\hline
\end{tabular}

Quelle: Eigene Berechnungen.

Die Summen der eingegangenen Angebote und ihre jeweiligen Anteile verdeutlichen den mit 60,4 \% überwiegenden Anteil der ökologischen Güter Grünland I an der Gesamtsumme von 33.747,91 € aller Angebote. Der Anteil der ökologischen Güter Grünland II beträgt 20,7 \% und jener der ökologischen Güter Grünland III 18,9 \%. Somit sind Angebote in Höhe von 20.385,57 € für das ökologische Gut Grünland I, in Höhe von 6.974,03 € für das ökologische Gut Grünland II und in Höhe von 6.388,31 € für das ökologische Gut Grünland III eingegangen.

\subsubsection{Angenommene Angebote}

Trotz des hohen Anteils der Angebote für ökologische Güter Grünland I sind in einem größeren Umfang als erwartet ökologisch höherwertige Schläge angeboten worden, was dazu geführt hat, dass von der durch den Regionalen Beirat Northeim eröffneten Option der Mittelverschiebung hin zu höherwertigen ökologischen Gütern Gebrauch gemacht wurde.

Aufgrund der Budgetrestriktion von $30.000 €$ konnten nicht alle Angebote angenommen werden, so dass letztlich 28 Landwirte mit insgesamt 159 Schlägen und einer Fläche von 288,56 ha einen Zuschlag erhalten haben. Von dem zur Verfügung stehenden Budget wurden Zuschläge in Höhe von insgesamt 29.463,17 € erteilt, von denen 16.100,04 € für Grünland I eingesetzt wurden, was einem Anteil von 54,6 \% entspricht. Alle Angebote für Grünland II im Wert von 6.974,03 € wurden angenommen und machen einen Anteil von 23,7 \% aus. Mit 21,7\% und einer Gesamtsumme von 6.388,30 € entfällt der geringste Anteil auf die ökologischen Güter Grünland III. Die Ergebnisse der angenommenen Angebote stellen sich für die ökologischen Güter Grünland I, II und III wie in Tabelle 7-9 abgebildet dar. 
Tab. 7-9: Ergebnisse der angenommenen Angebote für die ökologischen Güter Grünland I, II und III (2004/2005)

\begin{tabular}{|l|c|c|c|c|}
\hline & Preisspanne & Mittelwert & Median & Std.abw. \\
\hline Grünland I & $40-145 € /$ ha & $84,59 € /$ ha & $90,00 € /$ ha & $26,45 € /$ ha \\
\hline Grünland II & $55-300 € /$ ha & $141,75 € /$ ha & $140,00 € /$ ha & $59,55 € /$ ha \\
\hline Grünland III & $100-350 € /$ ha & $202,78 € /$ ha & $175,00 € /$ ha & $78,73 € /$ ha \\
\hline
\end{tabular}

Quelle: Eigene Berechnungen.

Hinsichtlich der in das Programm genommenen und damit potentiell für die Auszahlung relevanten Angebote zeigen sich für alle ökologischen Güter weiterhin die durch große Preisspannen und Standardabweichungen repräsentierten Angebotspreise, die im Rahmen differenzierter Vertragsabschlüsse berücksichtigt werden konnten.

\subsubsection{Auszahlung nach der Kontrolle}

Nachdem der Zeitraum bis einschließlich der Zuschlagserteilung betrachtet wurde, stellt sich nun die Frage, welche Angebote tatsächlich erfüllt wurden.

Hierbei ist positiv anzumerken, dass weiterhin bei allen Qualitätsstufen der ökologischen Güter große Preisspannen zu erkennen sind und diese durch den Einsatz eines Ausschreibungsverfahrens einbezogen werden konnten. Einen Überblick über die Ergebnisse der positiv kontrollierten und damit erfüllten Angebote gibt Tabelle 7-10.

Tab. 7-10: Ergebnisse der erfüllten Angebote für die ökologischen Güter Grünland I, II und III (2004/2005)

\begin{tabular}{|l|c|c|c|c|}
\hline & Preisspanne & Mittelwert & Median & Std.abw. \\
\hline Grünland I & $40-140 € /$ ha & $79,94 € /$ ha & $80,00 € /$ ha & $26,68 € /$ ha \\
\hline Grünland II & $55-250 € /$ ha & $136,67 € /$ ha & $130,00 € /$ ha & $54,79 € /$ ha \\
\hline Grünland III & $100-350 € /$ ha & $225,00 € /$ ha & $200,00 € /$ ha & $83,94 € /$ ha \\
\hline
\end{tabular}

Quelle: Eigene Berechnungen.

Die jeweilige Ausfallquote beträgt 20,2 \% für die ökologischen Güter Grünland I, 15,6 \% für die ökologischen Güter Grünland II und ein Drittel für die ökologischen Güter Grünland III. Dementsprechend wurden insgesamt 22.545,85 € für 227,89 ha ausgezahlt, von denen 11.852,90 € auf Grünland I entfallen sind, was einem Anteil von 52,6 \% entspricht. 5.911,24 € und somit 26,2 \% wurden für Grünland II gezahlt. 
Den geringsten Anteil machen auch hier mit 21,2 \% und 4.781,66 € die hochwertigsten ökologischen Güter Grünland III aus.

Bei der Bewertung der Angebotspreise wird deutlich, dass es bei allen ökologischen Gütern auch zum Zeitpunkt der Auszahlung zu differenzierten Angebotspreisen und mit einem höheren ökologischen Nutzen zu einem höheren Preisniveau gekommen ist. Daraus kann gefolgert werden, dass sich die Landwirte mit individuell verschiedenen Kostenstrukturen konfrontiert gesehen haben und in dieser ersten Ausschreibungsrunde eine Orientierung daran stattgefunden hat, was das Potential für eine praktische Zielerreichung von Ausschreibungen offenbart.

\subsubsection{Preiskalkulation und Bieterverhalten}

Neben der Betrachtung der Angebotspreise ist es bei der Bewertung von Ausschreibungen zur Honorierung von Umweltleistungen mit dem Ziel eines langfristig effizienten Einsatzes öffentlicher Mittel von großer Relevanz zu untersuchen, wie die Angebotspreise im Detail zu Stande gekommen sind.

Dazu wird nachfolgend die Preiskalkulation der Landwirte untersucht, wobei Fragen zum Bieterverhalten, zu möglichen Preisabsprachen und zu der Nutzung von ausschreibungsrelevanten Informationen thematisiert werden. Im Zuge dessen wird dort wo es angemessen ist - jeweils eine hinsichtlich der Betriebsform und der Art der Bewirtschaftung differenzierte Betrachtung der Befragungsergebnisse vorgenommen. Das Ziel ist es einerseits allgemeine Tendenzen aller an der Befragung teilnehmenden Landwirte $\mathrm{zu}$ erkennen und darüber hinaus $\mathrm{zu}$ untersuchen, ob Unterschiede zwischen Haupt- und Nebenerwerbslandwirten sowie ökologisch und konventionell wirtschaftenden Landwirten identifiziert werden können. Die im Folgenden herangezogenen Ergebnisse stammen aus einer schriftlichen Befragung von Landwirten, die im Rahmen der Ausschreibung 2004/2005 einen Zuschlag erhalten haben. ${ }^{131}$

\footnotetext{
${ }^{131}$ Der gesamte Fragebogen befindet sich im Anhang 4 der Arbeit.
} 


\subsubsection{Befragungsdesign und allgemeine Daten}

Nach dem Vertragsabschluss wurde den 28 Landwirten, welche für ein oder mehrere Angebote einen Zuschlag erhalten haben, im Herbst 2005 ein schriftlicher Fragebogen zugeschickt, der von 24 Landwirten ausgefüllt wurde, was einem Anteil von $85,7 \%$ entspricht. ${ }^{132}$

Bevor im Folgenden die Ergebnisse diskutiert werden, gilt es einführend einige Angaben zu den befragten Landwirten zu machen. Von den 24 an der Befragung teilnehmenden Landwirten bewirtschaften zwölf ihren Betrieb im Haupterwerb und ebenfalls zwölf im Nebenerwerb. Des Weiteren betreiben neun Landwirte eine ökologische und 15 Landwirte eine konventionelle Landwirtschaft. An Agrarumweltprogrammen haben bislang elf der 24 Landwirte mindestens einmal teilgenommen. Demzufolge zeigt sich die Heterogenität der in der Befragung relevanten Betriebe und es ist hervorzuheben, dass mit einem Anteil von 54,2 \% über die Hälfte der Landwirte bislang noch nicht an Agrarumweltprogrammen teilgenommen hat und durch das Forschungsprojekt erstmalig an einem in dieser Form potentiellen zukünftigen Agrarumweltprogramm Interesse bekundet hat.

\subsubsection{Ergebnisse}

Im Folgenden wird auf die einzelnen Ergebnisse der Preiskalkulation und des Bieterverhaltens der Landwirte eingegangen. Dazu wird eingangs jeweils die Frage in der im Fragebogen verwendeten Formulierung vorangestellt und sukzessive eine Diskussion der Ergebnisse vorgenommen. Abschließend erfolgt eine zusammenfassende Betrachtung.

\section{„Haben Sie sich im Rahmen der Angebotserstellung mit anderen Landwirten im Landkreis Northeim beraten?“}

Alle 24 Landwirte haben die Frage nach einer Beratung mit anderen Landwirten beantwortet, wobei ausdrücklich noch nicht nach einer Preisabsprache, sondern einem grundsätzlichen Informationsaustausch der Landwirte gefragt wurde.

Dies ist in einem relativ geringen Maß der Fall gewesen und mit 20,8 \% haben fünf der berücksichtigten Landwirte angegeben, dass sie sich derart untereinander beraten haben. Eine differenzierte Betrachtung, wie sie in Tabelle 7-11 dargestellt ist, zeigt

\footnotetext{
${ }^{132}$ Sofern keine abweichenden Angaben gemacht werden, beziehen sich die folgenden Ausführungen auf diese 24 Landwirte.
} 
dass hinsichtlich der ausschreibungsrelevanten Kommunikation der Bieter in der Modellregion weder zwischen konventionell und ökologisch wirtschaftenden Betrieben, noch zwischen Haupt- und Nebenerwerbsbetrieben wesentliche Unterschiede zu erkennen sind.

Tab. 7-11: Ausschreibungsspezifische Kommunikation der Bieter Differenziert nach Bewirtschaftungs- und Betriebsform (2004/2005)

\begin{tabular}{|l|c|r|}
\hline $\mathrm{n}=24$ & Ja & \multicolumn{1}{|c|}{ Nein } \\
\hline Gesamt & $5(20,8 \%)$ & $19(79,2 \%)$ \\
\hline Konventionell & $4(26,7 \%)$ & $11(73,3 \%)$ \\
\hline Ökologisch & $1(11,1 \%)$ & $8(88,9 \%)$ \\
\hline Haupterwerb & $2(16,7 \%)$ & $10(83,3 \%)$ \\
\hline Nebenerwerb & $3(25,0 \%)$ & $9(75,0 \%)$ \\
\hline
\end{tabular}

Quelle: Eigene Berechnungen.

Zusammenfassend ist in dieser ersten Ausschreibungsrunde noch keine umfassende Kommunikation zwischen den Bietern zu erkennen, was speziell vor dem Hintergrund möglicher Preisabsprachen und anderer wettbewerbsverzerrender Effekte positiv zu bewerten ist.

„Haben Sie sich im Rahmen der Angebotserstellung mit anderen Landwirten im Landkreis Northeim hinsichtlich des Angebotspreises abgesprochen?“

Eine bei der Bewertung von Ausschreibungen und ihrer praktischen Effizienz maßgebliche Frage ist, ob es zwischen den Bieter zu Preisabsprachen gekommen ist. Sollte dies der Fall sein, besteht die Gefahr, dass die individuellen Kostenunterschiede an Entscheidungsrelevanz verlieren und nicht mehr über den Angebotspreis signalisiert werden. Je nach dem Ausmaß der Preisabsprachen würde damit ein entscheidender Vorteil von Ausschreibungen gegenüber einer einheitlichen Prämienzahlung ganz oder teilweise verloren gehen.

Daher ist es sehr positiv zu bewerten, dass alle 24 Landwirte angegeben haben, sich im Rahmen der Angebotserstellung für die Ausschreibungsrunde 2004/2005 nicht mit anderen Landwirten hinsichtlich des Angebotspreises abgesprochen zu haben. Ein derartiges Ergebnis unterstreicht die bereits bei der Betrachtung der Angebotspreise angestellte Einschätzung, dass die Anzahl der an der Ausschreibung teilnehmenden Landwirte hinreichend groß war, um eine tatsächliche Konkurrenzsituation sicherzustellen. 
„Können Sie sich vorstellen, sich im Rahmen einer zukünftigen Ausschreibungsrunde mit anderen Landwirten im Landkreis Northeim hinsichtlich des Angebotspreises abzusprechen?“

Neben einer auf die erste Ausschreibung bezogenen Frage nach Preisabsprachen, wurde des Weiteren gefragt, ob sich die Landwirte vorstellen können, sich in einer zukünftigen Ausschreibungsrunde mit anderen Landwirten in der Modellregion abzusprechen.

Entgegen der Einschätzung für die erste Ausschreibung haben nun mit 10 Landwirten 41,7 \% angegeben, dass sie sich vorstellen können zukünftig derartige Preisabsprachen zu treffen. Differenziert nach konventionell und ökologisch wirtschaftenden Betrieben und Haupt- und Nebenerwerbsbetrieben sind - wie Tabelle 7-12 zeigt - keine bedeutenden Unterschiede $\mathrm{zu}$ erkennen und die Fragestellung einer zukünftigen Preisabsprache kann für alle Landwirte als nahezu gleichermaßen relevant angesehen werden.

Tab. 7-12: Einschätzung zukünftiger Preisabsprachen der Bieter - Differenziert nach Art der Bewirtschaftung und Betriebsform (2004/2005)

\begin{tabular}{|l|c|c|}
\hline $\mathrm{n}=24$ & Ja & Nein \\
\hline Gesamt & $10(41,7 \%)$ & $14(58,3 \%)$ \\
\hline Konventionell & $7(46,7 \%)$ & $8(53,3 \%)$ \\
\hline Ökologisch & $3(33,3 \%)$ & $6(66,7 \%)$ \\
\hline Haupterwerb & $5(41,7 \%)$ & $7(58,3 \%)$ \\
\hline Nebenerwerb & $5(41,7 \%)$ & $7(58,3 \%)$ \\
\hline
\end{tabular}

Quelle: Eigene Berechnungen.

Hier zeigt sich erwartungsgemäß die nicht unerhebliche Tendenz, dass Preisabsprachen von den Landwirten zunehmend in Betracht gezogen werden und damit die Gefahr von Effizienzverlusten einer Ausschreibung einhergeht. Um einer solchen Entwicklung entgegenzuwirken ist die durchführende Agrarverwaltung gefordert, das Ausschreibungsdesign vorausschauend so zu verändern, dass eventuelle Preisabsprachen keinen oder nur einen geringen Einfluss nehmen können und sich letztlich auch für die Landwirte als nicht sinnvoll erweisen. 
„Haben Sie im Rahmen der Angebotserstellung eine Beratung in Anspruch genommen und dabei Anhaltspunkte oder Vorgaben für Ihre Angebotspreise bekommen?“ / „Wenn Sie eine Beratung in Anspruch genommen und dabei Anhaltspunkte oder Vorgaben für Ihre Angebotspreise bekommen haben, haben Sie diese letztlich auch berücksichtigt?“

Die Frage nach der Inanspruchnahme einer Beratung wird hier unter dem Aspekt aufgegriffen, ob den teilnehmenden Landwirten dabei Anhaltspunkte oder Vorgaben für ihre Angebotspreise gemacht wurden und diese in der Angebotserstellung Berücksichtigung gefunden haben. Auch dadurch besteht die einer Situation von Preisabsprachen vergleichbare Problematik, dass es je nach Ausprägung und Höhe der Berücksichtigung exogener Vorgaben zu den oben erläuterten Effizienzverlusten kommt. Ebenso besteht die Gefahr, dass entgegen einer programmeinheitlichen Prämienzahlung - bedingt durch die freie Preisbildung bei einem Ausschreibungsverfahren - die Einflussmöglichkeiten von landwirtschaftlichen Beratungsinstitutionen zunehmen.

Von den an der Befragung teilnehmenden Landwirten haben sechs Landwirte im Rahmen der Angebotserstellung eine Beratung in Anspruch genommen und dabei entsprechende Vorgaben bekommen. Alle sechs Landwirte betreiben eine konventionelle Landwirtschaft, wobei jeweils drei Landwirte ihren Betrieb im Haupt- und im Nebenerwerb führen. Eine Berücksichtigung der Anhaltspunkte wurde jeweils von zwei Haupt- und zwei Nebenerwerbslandwirten vorgenommen, weshalb eine weitergehende differenzierte Darstellung an dieser Stelle nicht erfolgt.

Demzufolge sind bislang geringe Tendenzen zu erkennen, dass Vorgaben von Beratungsinstitutionen Einfluss auf die Preisbildung und die Ergebnisse der Ausschreibung genommen haben. Das Ergebnis, dass ausnahmslos konventionelle Betriebe Anhaltspunkte bekommen und berücksichtigt haben, spricht für ihren insgesamt höheren Organisationsgrad und ein größeres Einflusspotential entsprechender beratender Institutionen.

„Haben Sie bei der Kalkulation des Angebotspreises den von Ihnen zur Antragstellung benötigten Zeitaufwand bewertet und berücksichtigt?“

Die ebenfalls im Zuge der Ermittlung und Bewertung von Transaktionskosten der Landwirte angesprochene Frage, ob der für die Angebotsabgabe notwendige Zeitaufwand der Antragstellung auch bei der Preiskalkulation berücksichtigt wurde, 
wird hier bereits zur Analyse der von den Landwirten kalkulierten Kostenkomponenten aufgegriffen. So wurde die Frage von 24 Landwirten beantwortet und elf von ihnen haben angegeben, den Zeitaufwand kalkuliert und berücksichtigt zu haben, was einem Anteil von 45,8 \% entspricht.

Ein auf den ersten Blick unerwartetes Ergebnis, denn insbesondere vor dem Hintergrund einer unternehmerischen Kalkulation ist zu erwarten, dass auch die impliziten Kosten der Antragstellung Einfluss in die Preiskalkulation finden und durch den Preis gedeckt werden. Demgegenüber führt eine über die Produktionskosten hinausgehende Einbeziehung der Transaktionskosten zu einem höheren Angebotspreis und damit zu einer Verringerung der Wahrscheinlichkeit einer Zuschlagserteilung. Ein Argument, das speziell durch die in der erstmaligen Teilnahme an einem Ausschreibungsverfahren begründete Unsicherheit verstärkt wird.

Hinsichtlich der in Tabelle 7-13 verdeutlichten Differenzierung in konventionell und ökologisch wirtschaftende Betriebe kann kein wesentlicher Unterschied der Anbieter in der Modellregion ausgemacht werden. Ein deutlich anderes Bild zeigt sich bei der Betrachtung von Haupt- und Nebenerwerbsbetrieben. Hier haben 75,0 \% der Haupterwerbsbetriebe angegeben, den Zeitaufwand bzw. die Transaktionskosten der Angebotsabgabe bei der Preiskalkulation berücksichtigt zu haben. Im Gegensatz dazu haben lediglich 16,7 \% der im Nebenerwerb wirtschaftenden Landwirte diesen Zeitaufwand einkalkuliert.

Tab. 7-13: Berücksichtigung des Zeitaufwands der Angebotsabgabe bei der Preiskalkulation - Differenziert nach Bewirtschaftungs- und Betriebsform (2004/2005)

\begin{tabular}{|l|c|c|}
\hline $\mathrm{n}=24$ & Ja & Nein \\
\hline Gesamt & $11(45,8 \%)$ & $13(54,2 \%)$ \\
\hline Konventionell & $6(40,0 \%)$ & $9(60,0 \%)$ \\
\hline Ökologisch & $5(55,6 \%)$ & $4(44,4 \%)$ \\
\hline Haupterwerb & $9(75,0 \%)$ & $3(25,0 \%)$ \\
\hline Nebenerwerb & $2(16,7 \%)$ & $10(83,3 \%)$ \\
\hline
\end{tabular}

Quelle: Eigene Berechnungen.

Anhand dieser Ergebnisse lässt sich die grundsätzliche Relevanz von Transaktionskosten diskutieren, da sie von 54,2 \% der Landwirte nicht in die Angebotspreise einkalkuliert und entsprechend auf dem Markt nicht 
entscheidungsrelevant wurden. Eine Diskussion, die in Kapitel Acht ausführlich erfolgen wird.

„Haben Sie bei der Kalkulation des Angebotspreises über Ihre Kosten hinaus einen Gewinnzuschlag berücksichtigt?“ / „Wenn Sie bei der Kalkulation des Angebotspreises einen Gewinnzuschlag berücksichtigt haben, wie hoch ist dieser ungefähr? Prozent“

Hinsichtlich der durch ein Ausschreibungsverfahren $\mathrm{zu}$ induzierenden einzelwirtschaftlichen Kalkulation der Angebotspreise stellt sich die Frage, inwieweit neben der reinen Kostenkalkulation auch die Berücksichtigung eines zusätzlichen Gewinnzuschlags stattgefunden hat.

Die Frage wurde von 23 Landwirten beantwortet, von denen sechs Landwirte einen Gewinnzuschlag einkalkuliert haben, was einem Anteil von 26,1 \% entspricht. Im Gegensatz zu der oben betrachteten Frage der Einbeziehung des Zeitaufwands der Angebotsabgabe ergeben sich bei der Unterscheidung in konventionell und ökologisch wirtschaftende Betriebe ebenso wie bei einer Differenzierung in Hauptund Nebenerwerbsbetriebe keine grundlegend voneinander abweichenden Ergebnisse. Ein Gewinnzuschlag hat in dieser ersten Ausschreibungsrunde durchweg noch kaum Eingang in die Angebotspreise gefunden und die entsprechenden Befragungsergebnisse sind in Tabelle 7-14 dargestellt.

Tab. 7-14: Berücksichtigung eines Gewinnzuschlags bei der Preiskalkulation Differenziert nach Bewirtschaftungs- und Betriebsform (2004/2005)

\begin{tabular}{|l|c|c|}
\hline $\mathrm{n}=24$ & Ja & Nein \\
\hline Gesamt & $6(26,1 \%)$ & $17(73,9 \%)$ \\
\hline Konventionell & $4(28,6 \%)$ & $10(71,4 \%)$ \\
\hline Ökologisch & $2(22,2 \%)$ & $7(77,8 \%)$ \\
\hline Haupterwerb & $4(33,3 \%)$ & $8(66,7 \%)$ \\
\hline Nebenerwerb & $2(18,2 \%)$ & $9(81,8 \%)$ \\
\hline
\end{tabular}

Quelle: Eigene Berechnungen.

Im Anschluss wurden die einen Gewinnzuschlag berücksichtigenden Landwirte gebeten, anzugeben wie hoch dieser ungefähr in Prozent war. Die in Tabelle 7-15 abgebildeten Angaben reichen von $10 \%$ bis $50 \%$, wobei der Mittelwert 21,7 \%, der Median 17,5 \% und die Standardabweichung 15,1 \% beträgt. 
Tab. 7-15: Angaben zur Höhe des seitens der Landwirte berücksichtigten Gewinnzuschlags (2004/2005)

\begin{tabular}{|l|c|c|c|c|}
\hline $\mathrm{n}=6$ & Spanne & Mittelwert & Median & Std.abw. \\
\hline Gewinnzuschlag & $10-50 \%$ & $21,7 \%$ & $17,5 \%$ & $15,1 \%$ \\
\hline
\end{tabular}

Quelle: Eigene Berechnungen.

Demzufolge zeigt sich eine Differenz der Angaben und sowohl der Median als auch der Mittelwert des prozentualen Gewinnzuschlags bewegen sich um den bis Ende 2006 in Agrarumweltprogrammen noch berücksichtigten Wert der Anreizkomponente von $20 \%$.

„Werden Sie sich bei der Angebotsabgabe im Rahmen einer kommenden Ausschreibungsrunde an den Ihnen mittlerweile bekannten Angebotspreisen anderer Landwirte orientieren?“

Nach der Zuschlagserteilung wurde den teilnehmenden Landwirten in der Modellregion eine Information über alle eingegangenen Angebote zugeschickt. Dieses im Rahmen von Ausschreibungen vergaberechtlich festgelegte Vorgehen der Information der (unterlegenen) Anbieter über alle eingegangenen Angebote umfasst, anonymisiert und nach den ökologischen Gütern Grünland I, II und III getrennt, ausschließlich die jeweiligen Angebotspreise pro Hektar und keinerlei weitere Informationen; selbstverständlich auch nicht zu den gewählten Zuschlagsgrenzen. Als problematisch ist daran im Bereich der wiederholten Ausschreibung ökologischer Leistungen ein zu erwartendes Lernen aus den Angebotspreisen anderer Bieter und deren Vergleich mit den eigenen angenommenen und nicht angenommenen Angeboten anzusehen.

Hinsichtlich einer zukünftigen Ausrichtung an diesen Angebotspreisen haben sieben von 24 Landwirten angegeben, dass Sie sich daran orientieren werden. Mit einem Anteil von 70,8 \% schließen jedoch 17 Landwirte ein derartiges Vorgehen aus. Die durch Tabelle 7-16 veranschaulichte, in konventionell und ökologisch wirtschaftende Betriebe differenzierte, Betrachtung zeigt hierbei keine wesentliche Abweichung von dem Ergebnis aller Landwirte. Ein divergierendes Bild ist hingegen bei der Unterscheidung in Haupt- und Nebenerwerbsbetriebe zu erkennen. Seitens der Haupterwerbsbetriebe haben 50 \% der Landwirte angegeben, sich in einer zukünftigen Ausschreibungsrunde an den Angebotspreisen der anderen Landwirte zu orientieren. Demgegenüber haben lediglich 8,4 \% der Nebenerwerbsbetriebe eine 
derartige Einschätzung abgegeben, was auch hier bei Haupt- und Nebenerwerbsbetrieben unterschiedliche Preisfindungen erwarten lässt.

Tab. 7-16: Zukünftige Berücksichtigung anderer Angebotspreise - Differenziert nach Bewirtschaftungs- und Betriebsform (2004/2005)

\begin{tabular}{|l|c|c|}
\hline $\mathrm{n}=24$ & Ja & Nein \\
\hline Gesamt & $7(29,2 \%)$ & $17(70,8 \%)$ \\
\hline Konventionell & $4(26,7 \%)$ & $11(73,3 \%)$ \\
\hline Ökologisch & $3(33,3 \%)$ & $6(66,7 \%)$ \\
\hline Haupterwerb & $6(50,0 \%)$ & $6(50,0 \%)$ \\
\hline Nebenerwerb & $1(8,3 \%)$ & $11(91,7 \%)$ \\
\hline
\end{tabular}

Quelle: Eigene Berechnungen.

Zusammenfassend lassen die Ergebnisse den Schluss zu, dass bei den befragten Landwirten eine überwiegende Orientierung an ihren eigenen Kosten stattgefunden hat und zukünftig stattfinden wird. Jedoch zeigt sich auch hier, wie schon bei den vorherigen Fragen, dass in Teilen zukünftige Anpassungen der Angebotspreise zu erwarten sind.

„Wie werden Ihre Angebotspreise in einer zweiten Ausschreibungsrunde verglichen mit der letzten Ausschreibungsrunde - vermutlich ausfallen?“

Bedingt durch die Einbindung in ein zeitlich begrenztes Forschungsprojekt und ein nicht durch Projektmittel finanziertes Ausschreibungsbudget war zum Zeitpunkt dieser Befragung nicht sicher, ob eine zweite Ausschreibungsrunde stattfinden wird. Daher wurde versucht, eine erste grobe Orientierung zur Höhe der Angebotspreise in einer zukünftigen zweiten Ausschreibung durch eine entsprechende Frage an die teilnehmenden Landwirte zu erhalten.

Beantwortet haben die Frage 23 Landwirte, von denen ein Landwirt angegeben hat, dass seine zukünftigen Angebotspreise geringer sein werden als bisher. 18 Landwirte vermuten, dass sie ihre Angebotspreise nicht verändern und vier Landwirte, dass sie sie erhöhen werden. Die Angaben sind in Tabelle 7-17 differenziert nach Bewirtschaftungs- und Betriebsform der landwirtschaftlichen Betriebe dargestellt. Dabei zeigt sich erneut eine wesentliche Unterscheidung zwischen Haupt- und Nebenerwerbsbetrieben, da kein seinen Betrieb als Nebenerwerb führender Landwirt in der zukünftigen Ausschreibungsrunde einen höheren Angebotspreis in Aussicht stellt. 
Tab. 7-17: Vermutliche Höhe zukünftiger Angebotspreise - Differenziert nach Bewirtschaftungs- und Betriebsform

\begin{tabular}{|l|c|r|c|}
\hline $\mathrm{n}=23$ & Geringer & Gleich & Höher \\
\hline Gesamt & $1(4,3 \%)$ & $18(78,3 \%)$ & $4(17,4 \%)$ \\
\hline Konventionell & -- & $12(85,7 \%)$ & $2(14,3 \%)$ \\
\hline Ökologisch & $1(11,1 \%)$ & $6(66,7 \%)$ & $2(22,2 \%)$ \\
\hline Haupterwerb & -- & $8(66,7 \%)$ & $4(33,3 \%)$ \\
\hline Nebenerwerb & $1(9,1 \%)$ & $10(90,9 \%)$ & -- \\
\hline
\end{tabular}

Quelle: Eigene Berechnungen.

Ein mit 78,3 \% überwiegender Anteil der Landwirte, welcher keine nennenswerten Änderungen und ein Anteil von 17,4 \%, der einen Anstieg der Angebotspreise in Aussicht stellt, lassen darauf schließen, dass sich ein leichter Anstieg des Preisniveaus einstellen wird.

\subsubsection{Zusammenfassende Betrachtung}

Eine zusammenfassende Diskussion der Befragungsergebnisse hinsichtlich der Preiskalkulation und des Bieterverhaltens in der Ausschreibungsrunde 2004/2005 verdeutlicht, dass es bislang zu keinen Preisabsprachen und lediglich in geringem Umfang zu einer ausschreibungsrelevanten Kommunikation in der Modellregion gekommen ist. Gleichwohl zeigen sich erste Tendenzen in Richtung zukünftiger Preisabsprachen, deren tatsächliche Ausprägung anschließend anhand der Ergebnisse des zweiten Ausschreibungsverfahrens diskutiert wird.

Zur Berücksichtigung der Preiskalkulation der Landwirte wurden mit der Einbeziehung eines Gewinnzuschlags und der im Rahmen der Angebotserstellung relevanten Transaktionskosten über die Produktionskosten hinausgehende Aspekte betrachtet. Dabei konnte sowohl hinsichtlich eines Gewinnzuschlags als auch des Zeitaufwands der Angebotserstellung gezeigt werden, dass diese Bestandteile seitens der Landwirte bislang in einem relativ geringen Umfang einbezogen wurden. Als Erklärung sind vor allem die mit der erstmaligen Teilnahme an einem Ausschreibungsverfahren einhergehende Unsicherheit der Landwirte und zudem ein noch relativ gering ausgeprägtes unternehmerisches Kalkulieren zu nennen. Letzteres ist maßgeblich darin begründet, dass in bisherigen Agrarumweltprogrammen eine einzelwirtschaftliche Preiskalkulation, in dem Maß wie es bei einer Ausschreibung 
notwendig ist, noch nicht erforderlich war und zudem eine Anreizkomponente von 20 \% expliziten Gewinnüberlegungen entgegensteht.

Bezüglich der Differenzierung in konventionell und ökologisch wirtschaftende Betriebe hat sich gezeigt, dass keine grundlegenden Unterschiede der Preiskalkulation und des Bieterverhaltens identifiziert werden konnten. Ein anderes Bild verdeutlicht die Unterscheidung in Haupt- und Nebenerwerbsbetriebe. Landwirte, die ihren Betrieb im Nebenerwerb führen, haben in einem deutlich geringeren Umfang angegeben, den Zeitaufwand der Angebotsabgabe einbezogen zu haben und zukünftig andere Angebotspreise im Rahmen ihrer Preiskalkulation zu berücksichtigen. Zudem haben ausschließlich Nebenerwerbslandwirte angeführt, dass ihre Angebotspreise in einer zweiten Ausschreibung vermutlich nicht höher als in der ersten ausfallen werden.

Eine Einschätzung, die vermuten lässt, dass Nebenerwerbslandwirte aufgrund ihrer nicht ausschließlichen Abhängigkeit der durch die landwirtschaftliche Produktion erzielten Einnahmen geringere Angebotspreise abgeben und damit die Wahrscheinlichkeit der Zuschlagserteilung innerhalb eines Ausschreibungsverfahrens erhöhen können.

\subsection{Die Ausschreibung 2006}

Das zweite Ausschreibungsverfahren für ökologische Güter auf Grünlandflächen wurde im Jahr 2006 durchgeführt. Dabei wurde insbesondere das Ziel verfolgt, das theoretisch abgeleitete und sich innerhalb der Ausschreibung 2004/2005 als erfolgreich erwiesene Ausschreibungsdesign erneut einer praktischen Erprobung zu unterziehen und dahingehend $\mathrm{zu}$ analysieren, inwieweit sich die Vorteile dieser Ausschreibung für Umweltleistungen auch bei einer wiederholten Durchführung einstellen.

Nachfolgend wird in Abschnitt 7.2.1 auf grundlegende Aspekte, den zeitlichen Ablauf und die Beiratsentscheidung eingegangen. Im Anschluss liegt der Schwerpunkt auf den seitens der Landwirte kalkulierten Angebotspreisen, welche in den Abschnitten 7.2.2, 7.2.3 und 7.2.4 jeweils für die ökologischen Güter Grünland I, II und III dargestellt werden. Eine zusammenfassende Analyse der Angebotspreise 
für alle ökologischen Güter gibt Abschnitt 7.2.5, bevor abschließend in Abschnitt 7.2.6 die Preiskalkulation und das Bieterverhalten der Landwirte anhand einer schriftlichen Befragung thematisiert werden.

\subsubsection{Grundlagen, zeitlicher Ablauf und Beiratsentscheidung}

Bedingt durch eine kurzfristig erfolgte Sicherung der Finanzierung einer zweiten Ausschreibung und einem zum Herbst 2006 auslaufenden Forschungsprojekt wurde entgegen des ersten Ausschreibungsverfahrens eine wesentliche Änderung der Ausgestaltung vorgenommen.

Diese bezieht sich auf den Kreis der teilnahmeberechtigten Landwirte und es wurde für die Ausschreibung 2006 eine beschränkte Ausschreibung ${ }^{133}$ in dem Sinn durchgeführt, dass lediglich die 39 Landwirte in der Modellregion Berücksichtigung finden konnten, die bereits im Rahmen der Ausschreibung 2004/2005 ein Angebot abgegeben haben. Nachdem die Anpassung des Ausschreibungsverfahrens seitens des Regionalen Beirats Northeim beschlossen wurde, konnte im Anschluss am 23. Januar 2006 über den Nachfragekatalog beraten werden.

Hierbei hat sich das regionale Gremium einstimmig dazu entschlossen, erneut drei ökologische Güter Grünland nachzufragen, wobei die in der ersten Ausschreibung gewählte Güterdefinition beibehalten wurde. Hinsichtlich der Aufteilung des zur Verfügung stehenden Budgets in Höhe von $26.000 €^{134}$ wurde beschlossen, dass jeweils ein Drittel (rund $8.667 €$ ) für das ökologische Gut Grünland I, II und III verwendet werden soll. Damit wurde auf das Ergebnis der Ausschreibung 2004/2005 reagiert, in der ein relativ großer Anteil hochwertiger Umweltleistungen angeboten wurde.

Die Ausgestaltung der Unterlagen und die formalen Vorgaben an die Teilnahme wurden weitestgehend beibehalten und im Gegensatz zur ersten Ausschreibung lediglich dahingehend angepasst, dass zur Kennzeichnung der angebotenen Schläge das nunmehr eingeführte System der Feldblockidentifikatoren entsprechend der aktuellen Betriebskarte der Landwirtschaftskammer eingesetzt wurde. ${ }^{135}$

\footnotetext{
133 Die Beschränkung der Ausschreibung erfolgte in Anlehnung an die Regelungen zu beschränkten Ausschreibungen gemäß des $\S 3$ der Verdingungsverordnung für Bauleistungen (VOB).

${ }^{134}$ Das Budget wurde vom Landkreis Northeim, der Umweltlotterie „BINGO!“ und der KWS Saat AG zur Verfügung gestellt.

${ }^{135}$ Die gesamten Ausschreibungsunterlagen finden sich im Anhang 2 dieser Arbeit. Dort wird zudem eine umfassende Auflistung und Abgrenzung der ökologischen Güter gegeben.
} 
Durch die Beschränkung der Ausschreibung auf bereits mit dem Ausschreibungsverfahren vertraute Landwirte wurden keine Informationsveranstaltungen durchgeführt. Zudem erfolgte keine öffentliche Bekanntmachung der Ausschreibung und die Ausschreibungsunterlagen wurden den relevanten landwirtschaftlichen Betriebsleitern ab Mitte Februar unmittelbar zugeschickt, so dass sie bis zum Ablauf der Angebotsfrist am 31. März 2006 ihre Angebote abgeben konnten. Die eingegangenen Angebote unterlagen weiterhin einer für die ganze Modellregion Landkreis Northeim einheitlichen Ausschlussgrenze und die fristgerecht eingegangenen Angebote wurden seitens der Projektmitarbeiter im Rahmen der Zuschlagsfrist bis zum 07. April 2006 bewertet.

Als Zuschlagskriterium diente hierbei erneut aus ökologischer Sicht die Einstufung der Schläge in die Güterklassen Grünland I, Grünland II und Grünland III und aus ökonomischer Sicht der Angebotspreis pro Hektar, so dass jeweils bis zur Ausschöpfung der Teilbudgets die Zuschläge erteilt wurden.

Die Vertragslaufzeit betrug bedingt durch die oben erläuterten zeitlichen Restriktionen lediglich vier Monate. Hier ist erneut anzumerken, dass mit dem gewählten Vorgehen zum einen zumindest Anreize für den Erhalt bestehender Artenvielfalt gesetzt werden sollten und zudem vor allem die Möglichkeit ergriffen wurde, eine weitere praktische Erprobung eines Ausschreibungsverfahrens maßgeblich vor dem Hintergrund der Entwicklung der Angebotspreise vornehmen zu können.

Im Gegensatz zum Ausschreibungsverfahren 2004/2005 werden nachfolgend die Ergebnisse der Kontrolle der ökologischen Güter in Form der im August 2006 erfolgten Auszahlungen an die Landwirte nicht einbezogen. ${ }^{136}$ Der Schwerpunkt liegt demzufolge auf den Angebotspreisen der eingegangenen und der angenommenen Angebote sowie der Preiskalkulation und dem Bieterverhalten der Landwirte.

\footnotetext{
${ }^{136}$ Dies ist durch zeitliche Restriktionen innerhalb des Forschungsprojekts bedingt, die in der zweiten Ausschreibungsrunde keine umfassenden Kontrollen der Flächen zugelassen haben. Zudem ist anzumerken, dass bedingt durch die Beschränkung der Ausschreibung auf bereits ein Angebot abgebende Landwirte die Kontrollergebnisse der ersten Ausschreibung berücksichtigt werden konnten und für diese erneut angebotenen Flächen hier eine weitestgehende Vertragserfüllung unterstellt werden kann.
} 


\subsubsection{Ergebnisse - Ökologisches Gut Grünland I}

Beginnend werden die Angebotspreise der ökologischen Güter Grünland I betrachtet, wobei die ökologischen Mindestvoraussetzungen an die pflanzliche Biodiversität durch das Vorkommen von acht unterschiedlichen Kräuterarten je Kontrollparzelle zu charakterisieren sind. Dies stellt die relativ geringwertigste Artenvielfalt und demzufolge ebenfalls den geringsten ökologischen Nutzen innerhalb der ausgeschriebenen Umweltleistungen dar.

Nachfolgend werden zuerst alle fristgerecht eingegangenen und formal korrekten Angebote hinsichtlich der Angebotspreise pro Hektar betrachtet und im Anschluss die angenommenen Angebote thematisiert.

\subsubsection{Eingegangene Angebote}

Für das ökologische Gut Grünland I wurden von 26 Landwirten 216 Schläge im Umfang von 340,65 Hektar angeboten, von denen alle fristgerecht und formal korrekt eingegangen sind.

Die Angebotspreise pro Hektar umfassen mit einem minimalen Angebotspreis von $25 €$, einem maximalen Wert von $160 €$ und einer Standardabweichung von 29,47 € eine Spanne von $135 €$. Der sich aus diesen in Abbildung 7-8 dargestellten individuellen Angebotspreisen und der Häufigkeit ihres Vorkommens ergebende Mittelwert beträgt 93,94 €; der Median liegt bei $100 €$. 


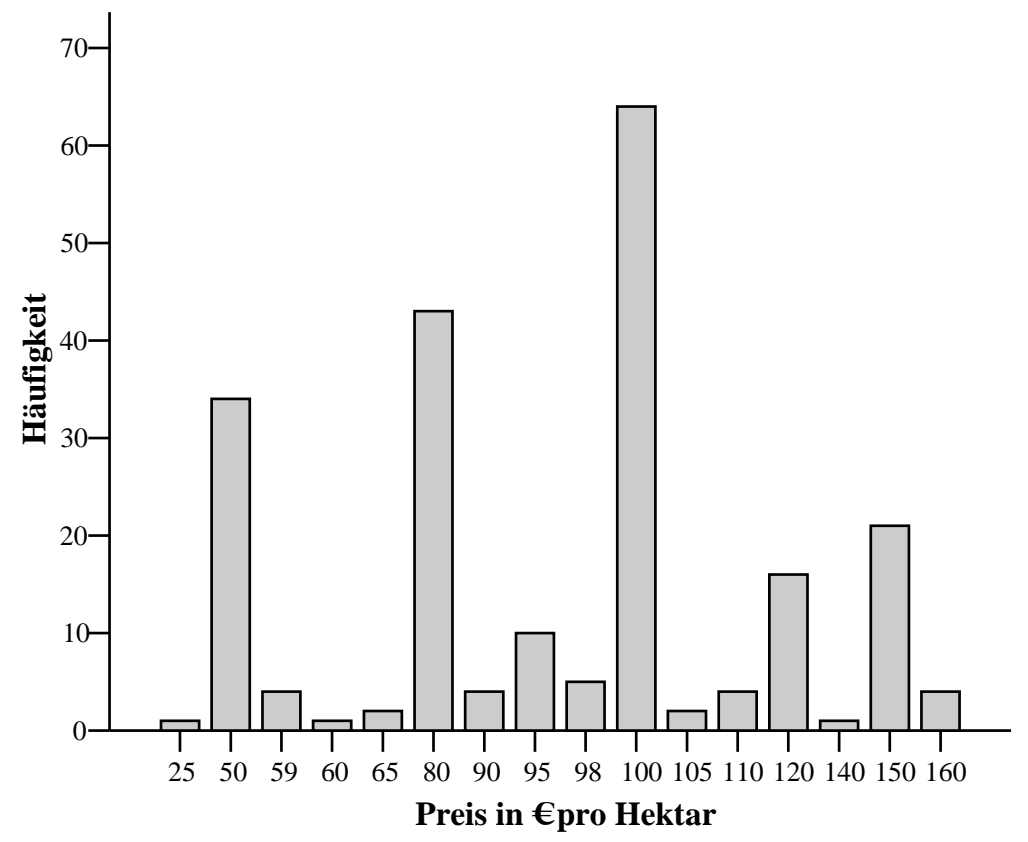

Abb. 7-8: Angebotspreise in $€$ pro Hektar der eingegangenen Angebote für das ökologische Gut Grünland I und Häufigkeit ihres Auftretens (2006)

Quelle: Eigene Darstellung nach eigenen Berechnungen.

Somit lassen die Ergebnisse weitreichende Unterschiede der Angebotspreise für eine identische Umweltleistung erkennen, wobei Häufungen vor allem in Höhe von $50 €$, $80 €$ und $100 €$ zu erkennen sind. Einen Überblick über die zentralen Ergebnisse der eingegangenen Angebote für die ökologischen Güter Grünland I gibt Tabelle 7-18.

Tab. 7-18: Ergebnisse der eingegangenen Angebote für die ökologischen Güter Grünland I (2006)

\begin{tabular}{|l|c|c|c|c|}
\hline $\mathrm{n}=216$ & Preisspanne & Mittelwert & Median & Std.abw. \\
\hline Grünland I & $25-160 € /$ ha & $93,94 € /$ ha & $100,00 € /$ ha & $29,47 € /$ ha \\
\hline
\end{tabular}
Quelle: Eigene Berechnungen.

Demzufolge zeigt sich die Differenzierung der Angebote ebenfalls bei der Betrachtung der Summen pro angebotenem Schlag, welche mit einem Mittelwert von $150,38 €$ und einem Median von 98,70 $€$ die Spanne von $13 €$ bis 2.173,50 $€$ umfassen. Die Gesamtsumme aller eingegangenen Angebote beträgt 32.482,39 € und liegt in einem hohen Maß über dem seitens des Regionalen Beirats Northeim beschlossenen Teilbudgets. 


\subsubsection{Angenommene Angebote}

Durch die Vorgabe, rund $8.667 €$ für die Angebote innerhalb der ökologischen Güter Grünland I aufzuwenden, konnten nicht alle Flächen angenommen werden. Insgesamt wurde 89 Angeboten von 10 Landwirten ein Zuschlag erteilt, was einer Gesamtfläche von 130,05 Hektar und einer Summe von 8.527,30 € entspricht. Die sich daraus ergebenden Summen pro angenommenem Schlag belaufen sich bei einem Minimum von $13 €$ und einem Maximum von 777,62 $€$ auf eine Spanne, die durch den Mittelwert 95,81 $€$ und den Median $65 €$ gekennzeichnet ist. Die Angebotspreise der angenommenen Angebote und die Häufigkeit ihres Auftretens verdeutlicht Abbildung 7-9.

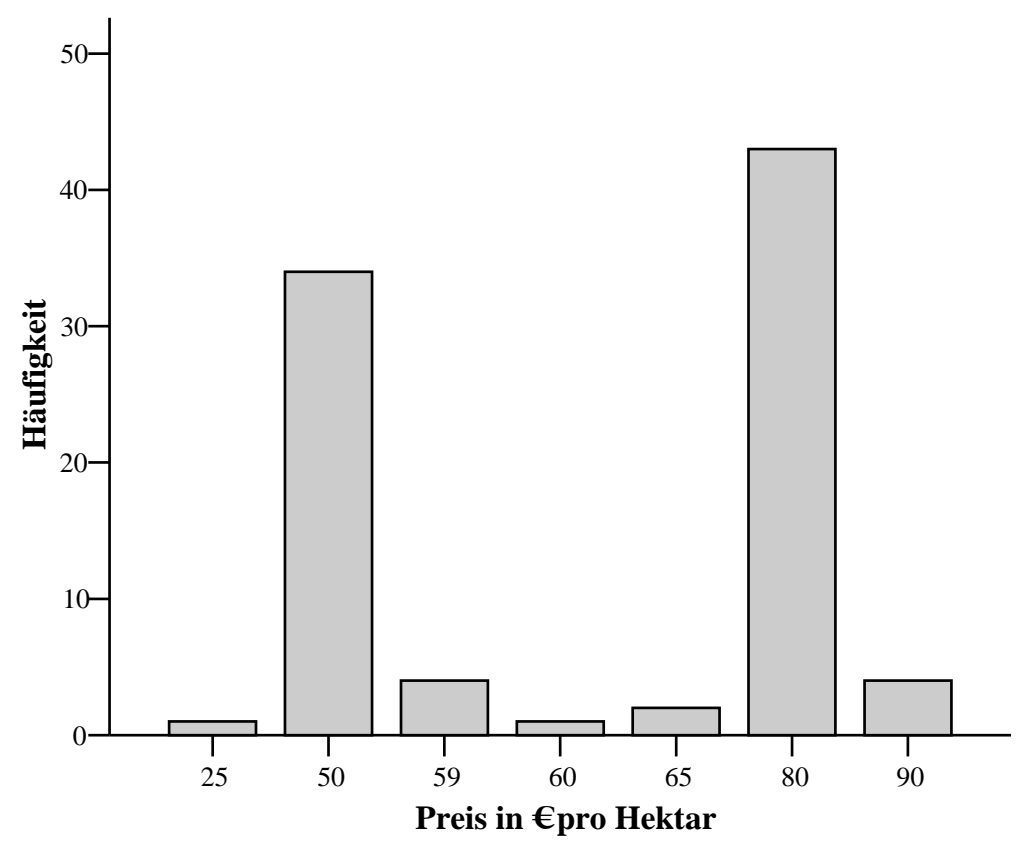

Abb. 7-9: Angebotspreise in $€$ pro Hektar der angenommenen Angebote für das ökologische Gut Grünland I und Häufigkeit ihres Auftretens (2006)

Quelle: Eigene Darstellung nach eigenen Berechnungen.

Hinsichtlich der Angebotspreise dieser angenommenen Angebote zeigt sich weiterhin grundsätzlich ihre durch den Einsatz eines Ausschreibungsverfahrens aufgedeckte und berücksichtigte Differenz mit einem minimalen Wert von $25 €$ und einem der Zuschlagsgrenze entsprechenden Maximum von $90 €$ pro Hektar. Die Standardabweichung beträgt 15,56 €, als Mittelwert hat sich ein Angebotspreis von $66,86 €$ herausgebildet und der Median liegt bei $80 €$.

Es wird gleichwohl deutlich, dass - bedingt durch das niedrige zur Verfügung stehende Teilbudget und die relativ geringe Anzahl der angenommenen Schläge - 
hier keine umfassende Vertragsdifferenzierung mehr vorgenommen werden konnte und die Angebotspreise zwei wesentliche Häufungen in Höhe von $50 €$ und $80 €$ aufweisen. Die Ergebnisse der angenommenen Angebote für die ökologischen Güter Grünland I fasst Tabelle 7-19 zusammen.

Tab. 7-19: Ergebnisse der angenommenen Angebote für die ökologischen Güter Grünland I (2006)

\begin{tabular}{|l|c|c|c|c|}
\hline $\mathrm{n}=89$ & Preisspanne & Mittelwert & Median & Std.abw. \\
\hline Grünland I & $25-90 € /$ ha & $66,86 € /$ ha & $80,00 € /$ ha & $15,56 € /$ ha \\
\hline
\end{tabular}
Quelle: Eigene Berechnungen.

Über die Betrachtung der Angebotspreise hinaus, kann für die Ausschreibung 2006 analog zu dem Vorgehen innerhalb der ersten Ausschreibungsrunde für die ökologischen Güter Grünland I ein praktischer Vergleich mit einer bestehenden Einheitsprämie vorgenommen werden. Hierzu wird als Referenzwert erneut die Prämienzahlung für die „Förderung extensiver Grünlandnutzung“ aus der „Richtlinie über die Gewährung von Zuwendungen für Niedersächsische Agrarumweltprogramme (NAU) 2005“ in Höhe von $103 €$ herangezogen.

Ein Vergleich dieser Prämienzahlung mit den Angebotspreisen in Höhe von $25 €$ bis $90 €$ pro Hektar zeigt, dass die innerhalb des Forschungsprojekts angenommene Fläche von 130,05 Hektar bei der Nutzung der Prämienzahlung ein Budget von 13.395,15 € erforderlich gemacht hätte. Demgegenüber konnte diese Fläche unter Nutzung einer Ausschreibung für 8.527,30 € und mit einer Einsparung in Höhe von 4.867,85 € bzw. 36,3 \% erreicht werden. Dem entspricht das Ergebnis, dass mit der Prämie von $103 €$ und dem Ausschreibungsbudget von 8.527,30 € nicht wie hier 130,05 Hektar, sondern lediglich eine Gesamtfläche von 82,79 Hektar hätten berücksichtigt werden können. Die Beispielrechnungen zeigen, dass in diesem Fall ein höherer ökologischer Nutzen durch ein Ausschreibungsverfahren zu einem geringeren Budget als bei einer Einheitsprämie erreicht wurde, was das ökonomische Potential auch von wiederholten Ausschreibungsverfahren verdeutlicht.

\subsubsection{Ergebnisse - Ökologisches Gut Grünland II}

Das ökologische Gut Grünland II ist durch die Mindestvoraussetzung von acht verschiedenen Kräuterarten pro Kontrollparzelle gekennzeichnet, wobei von diesen 
acht Arten oder zusätzlich dazu zwei Arten Bestandteil eines Zielartenkatalogs sein müssen. Die Anforderungen gehen über die für das ökologische Gut Grünland I hinaus und lassen innerhalb des Ausschreibungsverfahrens einen mittleren ökologischen Nutzen erwarten.

Im Folgenden werden zu Beginn alle fristgerecht und formal korrekt eingegangenen Angebote und im Anschluss die angenommenen Angebote hinsichtlich der Angebotspreise analysiert.

\subsubsection{Eingegangene Angebote}

Insgesamt wurden für das ökologische Gut Grünland II von 18 Landwirten 56 Schläge angebotenen und alle Angebote sind fristgerecht und formal korrekt eingegangen. Die Gesamtfläche dieser Angebote umfasst 82,58 Hektar.

Eine grafische Veranschaulichung der individuellen Angebotspreise pro Hektar und der Häufigkeit ihres Auftretens gibt Abbildung 7-10, wobei eine Häufung der Angebotspreise bei $130 €$ und $150 €$ zu erkennen ist.

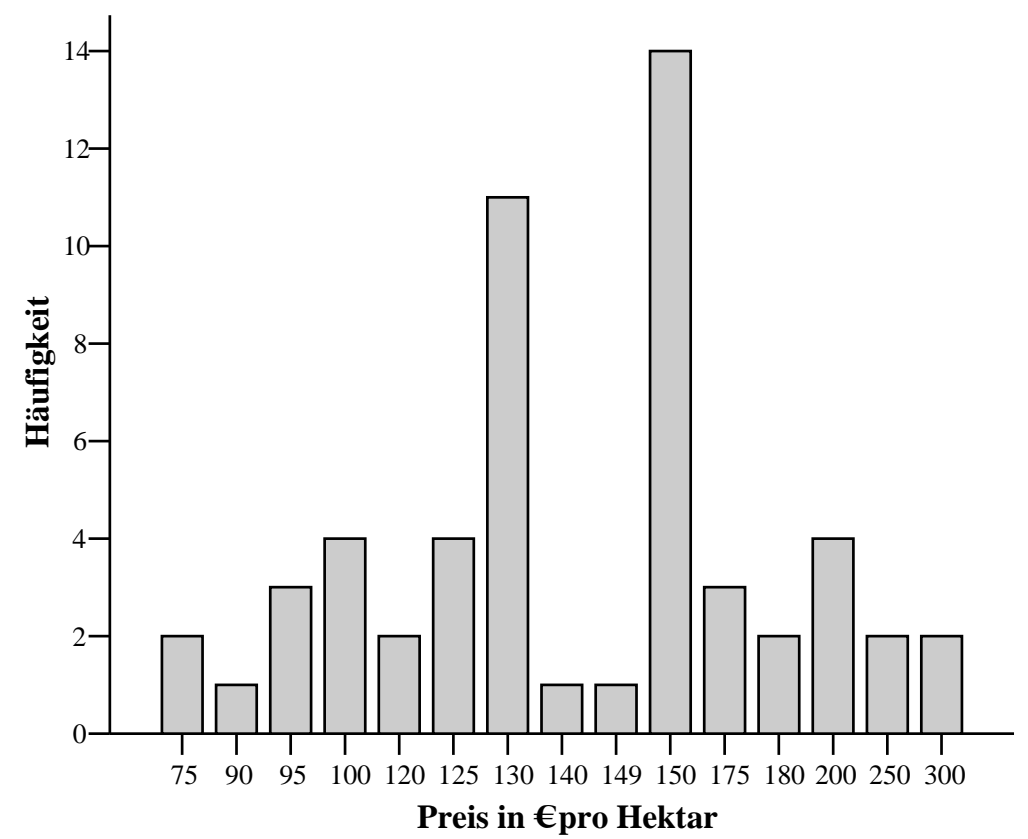

Abb. 7-10: Angebotspreise in $€$ pro Hektar der eingegangenen Angebote für das ökologische Gut Grünland II und Häufigkeit ihres Auftretens (2006)

Quelle: Eigene Darstellung nach eigenen Berechnungen.

Die auch hier realisierte große Differenziertheit der Angebotspreise zeigt sich durch die mit einem Mittelwert von 147,67 $€$, einem Median von 144,40 $€$ und einer Standardabweichung in Höhe von 46,92 € gekennzeichnete Preisspanne von $75 €$ bis 
$300 €$. Die hinsichtlich der Angebotspreise für die Umweltleistung Grünland II maßgeblichen Ergebnisse der eingegangenen Angebote sind in Tabelle 7-20 dargestellt.

Tab. 7-20: Ergebnisse der eingegangenen Angebote für die ökologischen Güter Grünland II (2006)

\begin{tabular}{|l|c|c|c|c|}
\hline $\mathrm{n}=56$ & Preisspanne & Mittelwert & Median & Std.abw. \\
\hline Grünland II & $75-300 € /$ ha & $147,67 € /$ ha & $144,50 € /$ ha & $46,92 € /$ ha \\
\hline
\end{tabular}
Quelle: Eigene Berechnungen.

Die Gesamtsumme der Angebote beträgt 11.489,32 € und liegt über dem seitens des Regionalen Beirats Northeim beschlossenen Teilbudgets von 8.667 €. Die Summen je Schlag belaufen sich im Mittelwert auf 205,17 € und der Median beträgt 152,86 €. Die ausgeprägte Differenz der angebotenen Flächen verdeutlicht die durch eine Standardabweichung von 163,59 € gekennzeichnete Spanne der Summen je Schlag, welche den Bereich von $30 €$ bis $624 €$ umfasst.

\subsubsection{Angenommene Angebote}

Insgesamt haben 52 Angebote von 17 Landwirten im Umfang von 76,80 Hektar einen Zuschlag erhalten. Im Rahmen dieser Zuschlagserteilung konnte die Beiratsentscheidung unter Bezugnahme auf die eingegangenen Angebote für die ökologischen Güter Grünland III angepasst und letztlich ein Budget von 9.843,82 € eingesetzt werden. Die Summen je angenommenem Schlag umfassen mit dem Mittelwert 189,30 $€$, dem Median 142,50 $€$ und einer Standardabweichung von 147,61 € die Spanne von $30 €$ bis 562,50 €. Die Angebotspreise pro Hektar und die Häufigkeit ihrer Berücksichtigung veranschaulicht Abbildung 7-11. 


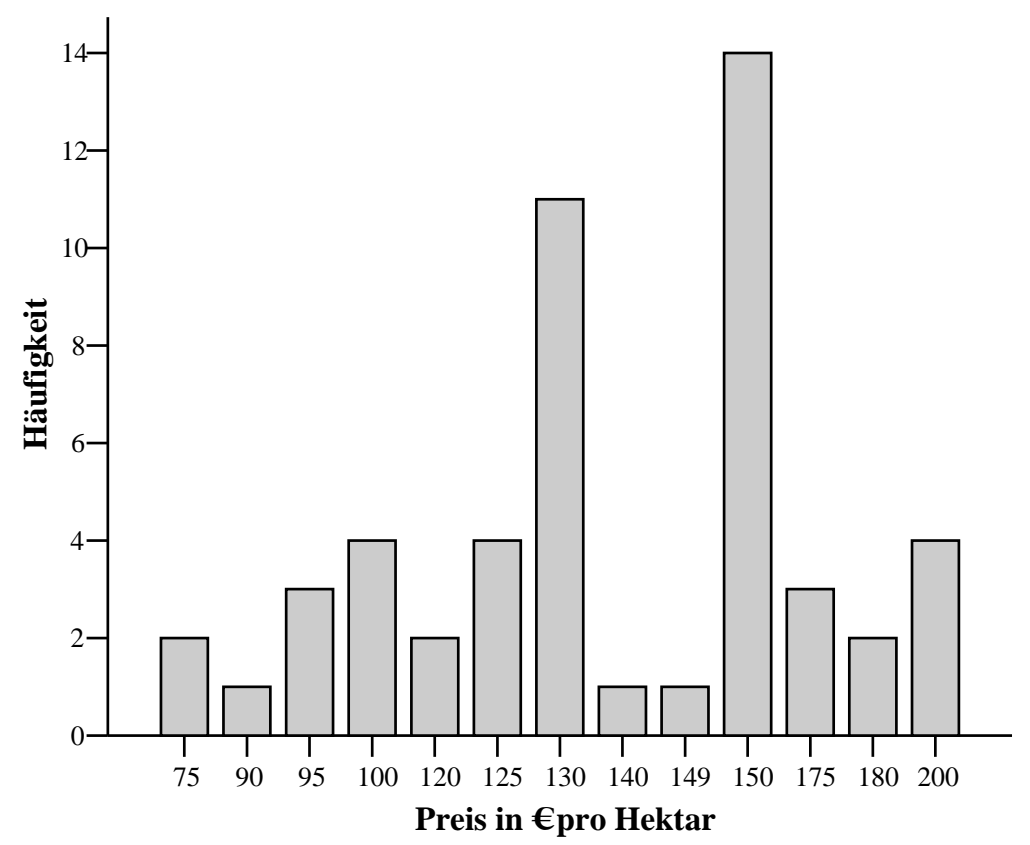

Abb. 7-11: Angebotspreise in $€$ pro Hektar der angenommenen Angebote für das ökologische Gut Grünland II und Häufigkeit ihres Auftretens (2006)

Quelle: Eigene Darstellung nach eigenen Berechnungen.

Die Angebotspreise pro Hektar der angenommenen Angebote umfassen eine Spanne von $75 €$ bis $200 €$ und sind durch eine Standardabweichung von 30,92 $€$ gekennzeichnet. Der Mittelwert beträgt 137,87 € und der Median liegt bei $130 €$. Die in Tabelle 7-21 zusammengefassten Ergebnisse verdeutlichen, dass es erneut gelungen ist, mit einem Ausschreibungsverfahren individuelle Preisunterschiede bei der Erbringung von Umweltleistungen aufzudecken und zu berücksichtigen. Die beiden Häufungen der Angebotspreise von $130 €$ und $150 €$ sind weiterhin existent, doch durch das relativ große Teilbudget konnte eine umfassende Vertragsdifferenzierung vollzogen werden.

Tab. 7-21: Ergebnisse der angenommenen Angebote für die ökologischen Güter Grünland II (2006)

\begin{tabular}{|l|c|c|c|c|}
\hline $\mathrm{n}=52$ & Preisspanne & Mittelwert & Median & Std.abw. \\
\hline Grünland II & $75-200 € /$ ha & $137,87 € /$ ha & $130,00 € /$ ha & $30,92 € /$ ha \\
\hline
\end{tabular}

Quelle: Eigene Berechnungen.

Ein praktischer Vergleich mit einer Einheitsprämie ist bedingt durch die spezifische Zieldefinition innerhalb des Forschungsprojekts problematisch, da derzeit auf kein übereinstimmendes Agrarumweltprogramm zurückgegriffen werden kann. Wird 
erneut die innerhalb der Agrarumweltförderung des Bundes im GAK-Rahmenplan 2005-2008 verankerte Prämienzahlung in Höhe von $130 €$ pro Hektar herangezogen, so zeigt sich, dass bei einer Prämienzahlung in Höhe von $130 €$ pro Hektar ein Budget von $9.984 €$ hätte aufgewendet werden müssen, um 76,80 Hektar artenreiche Grünlandfläche vertraglich zu binden; hier konnte dies für 9.843,82 € erfolgen.

\subsubsection{Ergebnisse - Ökologisches Gut Grünland III}

Innerhalb der simultan ausgeschriebenen Umweltleistungen stellt das ökologische Gut Grünland III den relativ größten ökologischen Nutzen in Aussicht und ist durch das Vorhandensein von acht unterschiedlichen Arten je Kontrollparzelle gekennzeichnet. Dabei müssen von diesen acht Arten oder zusätzlich dazu vier Arten Bestandteil eines Zielartenkatalogs sein.

Da die eingegangenen Angebote eine Gesamtsumme von 7.509,52 € aufgewiesen haben und infolgedessen das zur Verfügung stehende Teilbudget nicht überschritten wurde, konnten alle 23 Schläge von sieben Landwirten angenommen werden. Somit wird an dieser Stelle auf eine Unterteilung verzichtet und die eingegangenen und die angenommenen Angebote werden gemeinsam betrachtet. Die entsprechenden Schläge umfassen eine gesamte Grünlandfläche von 31,61 Hektar, wobei die Summen je Schlag mit einem Mittelwert von 326,50 $€$ und dem Median $318 €$ innerhalb der Spanne von $70 €$ bis $700 €$ liegen. Die sich daraus ergebende Standardabweichung beträgt 200,73 €. Die individuellen Angebotspreise pro Hektar der eingegangenen und angenommenen Angebote veranschaulicht Abbildung 7-12. 


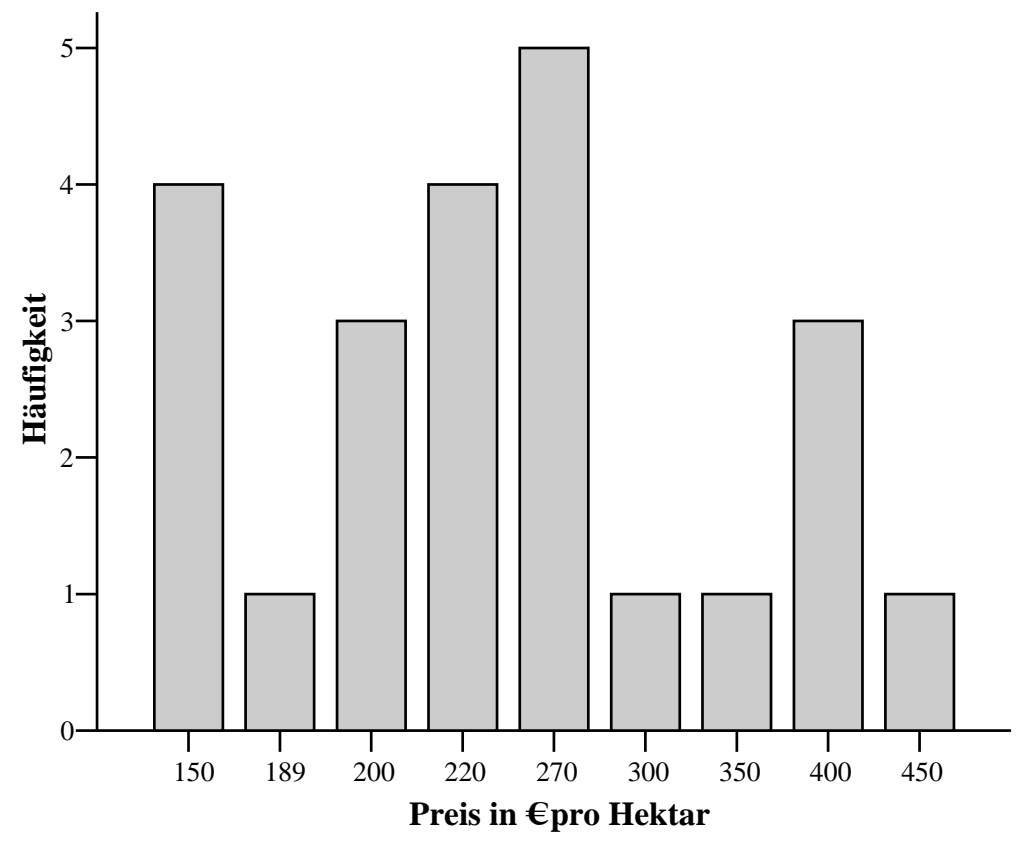

Abb. 7-12: Angebotspreise in $€$ pro Hektar der eingegangenen und angenommenen Angebote für das ökologische Gut Grünland III und Häufigkeit ihres Auftretens (2006)

Quelle: Eigene Darstellung nach eigenen Berechnungen.

Die Spanne der Angebotspreise pro Hektar umfasst mit dem Mittelwert 257,35 € und dem Median $220 €$ den Bereich von $150 €$ bis $450 €$. Neben dieser großen Preisspanne verdeutlicht die Standardabweichung von 89,34 $€$ die Verschiedenartigkeit der subjektiven Angebotspreise und demzufolge ebenfalls der Voraussetzungen zur Erbringung einer einheitlichen Umweltleistung.

Einen Überblick über die Angebotspreise der eingegangenen und angenommenen Angebote der ökologischen Güter Grünland III gibt Tabelle 7-22.

Tab. 7-22: Ergebnisse der eingegangenen und angenommenen Angebote für die ökologischen Güter Grünland III (2006)

\begin{tabular}{|l|c|c|c|c|}
\hline $\mathrm{n}=23$ & Preisspanne & Mittelwert & Median & Std.abw. \\
\hline Grünland III & $150-450 € /$ ha & $257,35 € /$ ha & $220,00 € /$ ha & $89,34 € /$ ha \\
\hline
\end{tabular}

Quelle: Eigene Berechnungen.

Ein praktischer Institutionenvergleich ist für das ökologische Gut Grünland III nicht möglich. Dies ist darin begründet, dass bislang noch kein Agrarumweltprogramm 
existiert, welches eine Prämienzahlung und eine vergleichbare Ergebnisorientierung entsprechend der hier honorierten Umweltleistung beinhaltet.

\subsubsection{Zusammenfassende Ergebnisse der Angebotspreise}

Mit dem Ziel eines direkten Vergleichs der Angebotspreise aller drei Qualitätsstufen von Umweltleistungen, erfolgt jeweils für die eingegangenen und die angenommenen Angebote eine zusammenfassende Gegenüberstellung der maßgeblichen Ausschreibungsergebnisse.

\subsubsection{Eingegangene Angebote}

Insgesamt haben 30 der 39 teilnahmeberechtigten Landwirte 295 Schläge angeboten, wobei alle Angebote fristgerecht und formal korrekt eingegangen sind. Die Gesamtfläche dieser Angebote beträgt 454,84 Hektar.

Eine Gegenüberstellung der Angebotspreise innerhalb der zweiten Ausschreibungsrunde in Tabelle 7-23 zeigt, dass es bei allen drei ökologischen Gütern zu differenzierten Angebotspreisen für eine jeweils identische Umweltleistung gekommen ist. Des Weiteren ist zu erkennen, dass mit einem steigenden ökologischen Nutzen - in Form einer höheren pflanzlichen Biodiversität - das Preisniveau ebenfalls angestiegen ist.

Tab. 7-23: Ergebnisse der eingegangenen Angebote für die ökologischen Güter Grünland I, II und III (2006)

\begin{tabular}{|l|c|c|c|c|}
\hline & Preisspanne & Mittelwert & Median & Std.abw. \\
\hline Grünland I & $25-160 € /$ ha & $93,94 € /$ ha & $100,00 € /$ ha & $29,47 € /$ ha \\
\hline Grünland II & $75-300 € /$ ha & $147,67 € /$ ha & $144,50 € /$ ha & $46,92 € /$ ha \\
\hline Grünland III & $150-450 € /$ ha & $257,35 € /$ ha & $220,00 € /$ ha & $89,34 € /$ ha \\
\hline
\end{tabular}

Quelle: Eigene Berechnungen.

Insgesamt sind Angebote im Umfang von 51.481,23 € eingegangen und hinsichtlich der Verteilung der Gesamtsumme auf die ökologischen Güter zeigt sich, dass mit einem Anteil von 63,1 \% und einer Summe von 32.482,39 € die überwiegenden Angebote für das relativ geringwertigste Gut Grünland I abgegeben wurden. Die einem mittleren ökologischen Nutzen entsprechende Güterstufe Grünland II nimmt mit der Summe von 11.489,32 € einen Anteil von 22,3 \% ein. Mit 7.509,52 € und 
einem Anteil von 14,6 \% wurden Umweltleistungen mit dem relativ größten ökologischen Nutzen - die Güter Grünland III - angeboten.

\subsubsection{Angenommene Angebote}

Mit der Summe aller Angebote von 51.481,23 € wurde das zur Verfügung stehende Gesamtbudget von $26.000 €$ überschritten und im Rahmen der Zuschlagserteilung konnten nicht alle angebotenen Flächen angenommen werden. Zudem galt es die seitens des Regionalen Beirats Northeim beschlossene Aufteilung der Finanzmittel zu berücksichtigen, bei der für jedes ökologische Gut ein Budget von rund $8.667 €$ vorgesehen war.

Demzufolge konnte 21 Teilnehmern ein Zuschlag für insgesamt 164 Schläge im Umfang von 25.880,64 € erteilt werden und die in der zweiten Ausschreibungsrunde vertraglich gebundene Gesamtfläche betrug 238,46 Hektar. Die Ergebnisse der angenommenen Angebote stellen sich hinsichtlich der Angebotspreise für die ökologischen Güter Grünland I, II und III wie in Tabelle 7-24 abgebildet dar.

Tab. 7-24: Ergebnisse der angenommenen Angebote für die ökologischen Güter Grünland I, II und III (2006)

\begin{tabular}{|l|c|c|c|c|}
\hline & Preisspanne & Mittelwert & Median & Std.abw. \\
\hline Grünland I & $25-90 € /$ ha & $66,86 € /$ ha & $80,00 € /$ ha & $15,56 € /$ ha \\
\hline Grünland II & $75-200 € /$ ha & $137,87 € /$ ha & $130,00 € /$ ha & $30,92 € /$ ha \\
\hline Grünland III & $150-450 € /$ ha & $257,35 € /$ ha & $220,00 € /$ ha & $89,34 € /$ ha \\
\hline
\end{tabular}

Quelle: Eigene Berechnungen.

Da für das ökologische Gut Grünland III mit einer Summe von 7.509,52 € in geringerem Umfang Angebote eingegangen sind als im Rahmen der Beiratsentscheidung beschlossen wurde, erfolgte die Zuschlagserteilung leicht abweichend und es wurden alle Angebote angenommen. Dies entspricht einem Anteil an der Gesamtsumme von 29,0 \%. Die frei gewordenen Mittel wurden in das Budget des ökologischen Guts Grünland II überführt und es konnten hier Angebote im Umfang von 9.843,82 € angenommen werden, was einem Anteil von 38,0 \% entspricht. Für die Güterklasse Grünland I wurden 8.527,30 € und 33,0 \% des gesamten Ausschreibungsbudgets aufgewendet.

Anhand der Angebotspreise ist zu erkennen, dass auch in der zweiten Ausschreibung eine umfassende Vertragsdifferenzierung hinsichtlich der Angebotspreise 
vorgenommen werden konnte und mit einem höheren ökologischen Nutzen ebenfalls das Preisniveau angestiegen ist.

\subsubsection{Preiskalkulation und Bieterverhalten der Landwirte}

Analog zum Ausschreibungsverfahren 2004/2005 werden neben den Angebotspreisen weitere Aspekte in die Analyse einbezogen. Dazu wurde eine schriftliche Befragung der innerhalb der Ausschreibung 2006 teilnahmeberechtigten Landwirte durchgeführt, um Erkenntnisse sowohl über die Preiskalkulation als auch das Bieterverhalten $\mathrm{zu}$ erlangen und diese zur Bewertung des Ausschreibungsverfahrens heranzuziehen.

Im Folgenden wird erneut das Vorgehen gewählt, bei der Diskussion der Befragungsergebnisse eine Differenzierung in Haupt- und Nebenerwerbslandwirte einerseits und konventionell und ökologisch wirtschaftende Landwirte andererseits vorzunehmen.

Hierbei ist anzumerken, dass zum Zeitpunkt der Befragung bereits absehbar war, dass eine erneute Ausschreibung von ökologischen Gütern auf Grünlandflächen innerhalb der geplanten Projektphase 2007 bis 2009 nicht erfolgen wird, sondern die Ausschreibung von Umweltleistungen auf Ackerflächen beabsichtigt ist. Im Zuge dessen und bedingt durch den Zeitpunkt der Befragung vor der Zuschlagserteilung wurde hier auf zwei Fragen zu zukünftigen Ausschreibungsrunden für Grünlandflächen - wie sie noch im Rahmen des ersten Ausschreibungsverfahrens gestellt wurden - verzichtet. ${ }^{137}$

\subsubsection{Befragungsdesign und allgemeine Daten}

Diese insgesamt dritte schriftliche Befragung hat parallel zur Angebotsfrist der Ausschreibung 2006 im Zeitraum Mitte Februar bis Ende März 2006 stattgefunden. Dazu haben alle 39 im Rahmen der beschränkten Ausschreibung teilnahmeberechtigten Landwirte zusammen mit den Ausschreibungsunterlagen - in

\footnotetext{
${ }^{137}$ Somit entfallen die folgenden Fragen: „Werden Sie sich bei der Angebotsabgabe im Rahmen einer kommenden Ausschreibungsrunde an den Ihnen mittlerweile bekannten Angebotspreisen anderer Landwirte orientieren?“, „Wie werden Ihre Angebotspreise in einer zweiten Ausschreibungsrunde verglichen mit der letzten Ausschreibungsrunde - vermutlich ausfallen?“.
} 
einem separaten Umschlag - einen Fragebogen ${ }^{138}$ zugeschickt bekommen, welcher von 27 Landwirten ausgefüllt und zurückgesandt wurde.

Dies entspricht einem Anteil von 69,2 \%, wobei 25 Landwirte ebenfalls im Zuge des Ausschreibungsverfahrens ein Angebot abgegeben haben. Diese 25 Landwirte stellen die für die weiteren Betrachtungen relevanten Landwirte dar und von den insgesamt 30 Landwirten, die ein Angebot abgegeben haben, sind demzufolge 83,3 \% in dieser Befragung repräsentiert. ${ }^{139}$ Die 25 Betriebe sind hinsichtlich ihrer Betriebsform dadurch gekennzeichnet, dass neun Landwirte ihren Betrieb im Haupterwerb und 16 im Nebenerwerb führen. Eine ökologische Landwirtschaft betreiben neun Landwirte und 16 Landwirte bewirtschaften ihre Flächen konventionell.

\subsubsection{Ergebnisse}

Nachfolgend werden die Befragungsergebnisse zur Preiskalkulation und zum Bieterverhalten der Landwirte betrachtet und im Zuge dessen wird eingangs jeweils die Frage in der im Fragebogen verwendeten Formulierung vorangestellt.

\section{„Haben Sie sich im Rahmen der Angebotserstellung mit anderen Landwirten im Landkreis Northeim beraten?“}

Die Frage nach einer Beratung mit anderen Landwirten und im Zuge dessen einer grundsätzlichen ausschreibungsrelevanten Kommunikation in der Modellregion wurde von allen 25 Landwirten beantwortet.

Die in Tabelle 7-25 dargestellten Antworten zeigen, dass es in der zweiten Ausschreibung in sehr geringem Umfang zu einer Kommunikation der Bieter gekommen ist.

Tab. 7-25: Ausschreibungsspezifische Kommunikation der Bieter Differenziert nach Bewirtschaftungs- und Betriebsform (2006)

\begin{tabular}{|l|c|c|}
\hline $\mathrm{n}=25$ & Ja & Nein \\
\hline Gesamt & $3(12,0 \%)$ & $22(88,0 \%)$ \\
\hline Konventionell & $1(6,3 \%)$ & $15(93,8 \%)$ \\
\hline Ökologisch & $2(22,2 \%)$ & $7(77,8 \%)$ \\
\hline Haupterwerb & $2(22,2 \%)$ & $7(77,8 \%)$ \\
\hline Nebenerwerb & $1(6,3 \%)$ & $15(93,8 \%)$ \\
\hline
\end{tabular}

Quelle: Eigene Berechnungen.

\footnotetext{
${ }^{138}$ Der gesamte Fragebogen findet sich im Anhang 5 dieser Arbeit.

${ }^{139}$ Sofern keine anderen Angaben gemacht werden, beziehen sich alle nachfolgenden Ausführungen auf diese 25 Landwirte.
} 
Eine darüber hinausgehende Betrachtung zeigt, dass weder zwischen konventionell und ökologisch wirtschaftenden Betrieben noch zwischen Haupt- und Nebenerwerbsbetrieben wesentliche Unterschiede zu erkennen sind.

„Haben Sie sich im Rahmen der Angebotserstellung mit anderen Landwirten im Landkreis Northeim hinsichtlich des Angebotspreises abgesprochen?“

Die direkte Frage nach Preisabsprachen wurde von allen 25 Landwirten beantwortet und lediglich ein ökologisch wirtschaftender Nebenerwerbsbetrieb hat angegeben, sich hinsichtlich der Angebotspreise mit anderen Bietern abgesprochen zu haben. Folglich kann an dieser Stelle auf eine weitergehende differenzierte Darstellung verzichtet und resümiert werden, dass es in der zweiten Ausschreibung zu keinen bedeutsamen und sich negativ auf die Ausschreibungsergebnisse auswirkenden Preisabsprachen gekommen ist. Eine Einschätzung, die neben den Befragungsergebnissen vor allem auch durch die oben betrachteten Angebotspreise der eingegangenen Angebote gestützt wird.

„Können Sie sich vorstellen, sich im Rahmen einer zukünftigen Ausschreibungsrunde mit anderen Landwirten im Landkreis Northeim hinsichtlich des Angebotspreises abzusprechen?“

Alle 25 Landwirte haben diesbezüglich eine Einschätzung abgegeben und mit 10 Landwirten hat ein Anteil von 40 \% angegeben, dass eine künftige Preisabsprache grundsätzlich in Betracht gezogen wird. Die Befragungsergebnisse sind sowohl für alle Landwirte als auch differenziert nach Art der Bewirtschaftung und Betriebsform in Tabelle 7-26 dargestellt.

Tab. 7-26: Einschätzung zukünftiger Preisabsprachen - Differenziert nach Art der Bewirtschaftung und Betriebsform (2006)

\begin{tabular}{|l|r|r|}
\hline $\mathrm{n}=25$ & Ja & Nein \\
\hline Gesamt & $10(40,0 \%)$ & $15(60,0 \%)$ \\
\hline Konventionell & $6(37,5 \%)$ & $10(62,5 \%)$ \\
\hline Ökologisch & $4(44,4 \%)$ & $5(55,6 \%)$ \\
\hline Haupterwerb & $5(55,6 \%)$ & $4(44,4 \%)$ \\
\hline Nebenerwerb & $5(31,3 \%)$ & $11(68,8 \%)$ \\
\hline
\end{tabular}

Quelle: Eigene Berechnungen. 
Hinsichtlich einer sowohl in konventionell und ökologisch wirtschaftende als auch in Haupt- und Nebenerwerbsbetriebe unterschiedenen Betrachtung zeigt sich, dass einzig die Haupterwerbslandwirte von dem Trend aller Landwirte abweichen und hier eine Mehrzahl von 55,6 \% Preisabsprachen zukünftig für realistisch erachtet. Diese Ergebnisse sprechen erneut für den hohen Organisationsgrad von Haupterwerbsbetrieben und ein entsprechendes Potential einer ausschreibungsrelevanten Kommunikation bis hin zu Preisabsprachen.

„Haben Sie im Rahmen der Angebotserstellung eine Beratung in Anspruch genommen und dabei Anhaltspunkte oder Vorgaben für Ihre Angebotspreise bekommen?“ / „Wenn Sie eine Beratung in Anspruch genommen und dabei Anhaltspunkte oder Vorgaben für Ihre Angebotspreise bekommen haben, haben Sie diese letztlich auch berücksichtigt?“

Neben den Preisabsprachen und einer Kommunikation der Bieter besteht eine weitere Einflussmöglichkeit für ein Abweichen von einer auf die individuellen Kosten bezogenen Preiskalkulation in Form exogener Preisvorgaben seitens einer Beratungsinstitution.

Die Frage wurde von allen 25 Landwirten beantwortet und zwei Landwirte haben angegeben, im Rahmen der Angebotserstellung eine Beratung in Anspruch genommen zu haben. Im Zuge dessen hat ein konventionell wirtschaftender Haupterwerbslandwirt Anhaltspunkte für Angebotspreise bekommen und angegeben, diese bei seiner Angebotserstellung nicht berücksichtigt zu haben. Demzufolge wird keine weitere differenzierte Betrachtung vorgenommen und es ist festzuhalten, dass sowohl der Beratungsbedarf innerhalb des zweiten Ausschreibungsverfahrens als auch der Einfluss von Beratungsinstitutionen als $\mathrm{zu}$ vernachlässigend gering einzuschätzen ist.

„Haben Sie bei der Kalkulation des Angebotspreises den von Ihnen zur Antragstellung benötigten Zeitaufwand bewertet und berücksichtigt?“

Insgesamt haben 25 Landwirte die Frage beantwortet. Davon haben 12 Landwirte angegeben, den Zeitaufwand der Angebotsabgabe bei der Kalkulation der Angebotspreise einbezogen zu haben, was einem Anteil von 48,0 \% entspricht. Die in Tabelle 7-27 aufgezeigte Differenzierung in Haupt- und Nebenerwerbslandwirte zeigt keinen wesentlichen Unterschied der Bieter. Ein abweichendes Bild offenbart 
die Betrachtung von konventionell und ökologisch wirtschaftenden Landwirten. Hier haben 62,5 \% der konventionell wirtschaftenden Landwirte angegeben, den Zeitaufwand bzw. die Transaktionskosten der Angebotsabgabe bei der Preiskalkulation berücksichtigt zu haben. Im Gegensatz dazu haben lediglich 22,2 \% der ökologisch wirtschaftenden Landwirte eine entsprechende Antwort gegeben.

Tab. 7-27: Berücksichtigung des Zeitaufwands der Angebotsabgabe bei der Preiskalkulation - Differenziert nach Bewirtschaftungs- und Betriebsform (2006)

\begin{tabular}{|l|c|c|}
\hline $\mathrm{n}=25$ & Ja & Nein \\
\hline Gesamt & $12(48,0 \%)$ & $13(52,0 \%)$ \\
\hline Konventionell & $10(62,5 \%)$ & $6(37,5 \%)$ \\
\hline Ökologisch & $2(22,2 \%)$ & $7(77,8 \%)$ \\
\hline Haupterwerb & $4(44,4 \%)$ & $5(55,6 \%)$ \\
\hline Nebenerwerb & $8(50,0 \%)$ & $8(50,0 \%)$ \\
\hline
\end{tabular}

Quelle: Eigene Berechnungen.

Es zeigt sich demzufolge auch im Rahmen des zweiten Ausschreibungsverfahrens eine von weniger als der Hälfte der Landwirte erfolgte Berücksichtigung der einzelwirtschaftlichen Transaktionskosten der Angebotserstellung. Eine abschließende Diskussion ihrer Relevanz wird in Kapitel Acht unter Rückgriff auf die Ermittlung und Bewertung der Transaktionskosten einbezogen.

„Haben Sie bei der Kalkulation des Angebotspreises über Ihre Kosten hinaus einen Gewinnzuschlag berücksichtigt?“ / „Wenn Sie bei der Kalkulation des Angebotspreises einen Gewinnzuschlag berücksichtigt haben, wie hoch ist dieser ungefähr? Prozent"

Auf der Grundlage der Antworten von 25 Landwirten zeigt sich, dass insgesamt fünf Landwirte einen Gewinnzuschlag einkalkuliert haben, was einem Anteil von 20,0 \% entspricht. Die in Tabelle 7-28 dargestellte Differenzierung hinsichtlich der Bewirtschaftungs- und Betriebsform lässt keine grundlegenden Unterschiede erkennen und zeigt für alle Landwirte die tendenziell gering ausgeprägte Berücksichtigung expliziter Gewinnzuschläge. 
Tab. 7-28: Berücksichtigung eines Gewinnzuschlags bei der Preiskalkulation Differenziert nach Bewirtschaftungs- und Betriebsform (2006)

\begin{tabular}{|l|c|c|}
\hline $\mathrm{n}=25$ & Ja & Nein \\
\hline Gesamt & $5(20,0 \%)$ & $20(80,0 \%)$ \\
\hline Konventionell & $3(18,8 \%)$ & $13(81,3 \%)$ \\
\hline Ökologisch & $2(22,2 \%)$ & $7(77,8 \%)$ \\
\hline Haupterwerb & $3(33,3 \%)$ & $6(66,7 \%)$ \\
\hline Nebenerwerb & $2(12,5 \%)$ & $14(87,5 \%)$ \\
\hline
\end{tabular}

Quelle: Eigene Berechnungen.

Eine weitergehende Frage zur Höhe des Gewinnzuschlags in Prozent wurde den fünf einen Gewinnzuschlag berücksichtigenden Landwirten gestellt. Hierbei haben alle fünf Landwirte eine Antwort gegeben und es zeigt sich in der zweiten Ausschreibung mit einem Gewinnzuschlag in der Spanne von $10 \%$ bis $15 \%$, bei einem Mittelwert von 11,2 \%, einem Median von $10 \%$ und der Standardabweichung von 2,5 \% ein sehr einheitliches Bild. Die entsprechenden Ergebnisse sind in Tabelle 7-29 dargestellt.

Tab. 7-29: Angaben zur Höhe des seitens der Landwirte berücksichtigten Gewinnzuschlags (2006)

\begin{tabular}{|l|c|c|c|c|}
\hline $\mathrm{n}=5$ & Spanne & Mittelwert & Median & Std.abw. \\
\hline Gewinnzuschlag & $10-15 \%$ & $11,2 \%$ & $10,0 \%$ & $2,5 \%$ \\
\hline
\end{tabular}

Quelle: Eigene Berechnungen.

Die in der zweiten Ausschreibung ebenfalls gering ausgeprägte Berücksichtigung eines Gewinnzuschlags lässt erneut vor allem zwei Erklärungen zu. Zum einen ist zu vermuten, dass die bisherige Ausgestaltung von Agrarumweltprogrammen in Form einheitlicher Kompensationszahlungen der expliziten Berücksichtigung einer eigenverantwortlichen Gewinnkalkulation entgegensteht. Zum anderen ist, wie oben bereits angesprochen wurde, innerhalb einer Ausschreibung die Situation gegeben, dass mit einem höheren Angebotspreis die Wahrscheinlichkeit einer Zuschlagserteilung abnimmt. In Folge der damit verbundenen Unsicherheit ist zu erwarten, dass dies eine Erhöhung des Angebotspreises durch die Einbeziehung eines Gewinnzuschlags auch in der zweiten Ausschreibungsrunde weitestgehend verhindert hat. Eine Einschätzung, die ebenfalls durch die relativ moderaten Gewinnzuschläge gestützt wird. 


\subsubsection{Zusammenfassende Betrachtung}

Die zusammenfassende Diskussion der Befragungsergebnisse verdeutlicht, dass es zu einer geringen ausschreibungsrelevanten Kommunikation der Bieter in der Modellregion gekommen ist. Perspektivisch zeigen sich Tendenzen in Richtung zukünftiger Preisabsprachen, wobei es als unsicher anzusehen ist, in welchem Maß entsprechende Handlungen sich tatsächlich einstellen werden.

Bei der Preiskalkulation der Landwirte ist in der zweiten Ausschreibung im Hinblick auf die Einbeziehung eines Gewinnzuschlags und des im Rahmen der Angebotserstellung anfallenden Zeitaufwands zu erkennen, dass beide Aspekte erneut lediglich in einem geringen Umfang Eingang in die Angebotspreise gefunden haben. Als Erklärung sind weiterhin die im Zuge der Teilnahme an einem Ausschreibungsverfahren vorherrschende Konkurrenzsituation um ein beschränktes Budget und die damit verbundene Unsicherheit eines Vertragsabschlusses zu nennen. Zudem ist mit einem durch die Einbeziehung über die Produktionskosten hinausgehender Bestandteile steigenden Angebotspreis grundsätzlich eine geringere Wahrscheinlichkeit der Zuschlagserteilung verbunden.

Bezüglich einer Unterscheidung in konventionell und ökologisch wirtschaftende Landwirte ist innerhalb der wiederholten Ausschreibung bei der Einbeziehung eines Gewinnzuschlags keine wesentliche Abweichung $\mathrm{zu}$ identifizieren. Ein unterschiedliches Ergebnis hat sich für die Berücksichtigung des Zeitaufwands der Angebotserstellung herausgestellt, indem dieser einzig seitens der konventionell wirtschaftenden Landwirte von mehr als der Hälfte einbezogen wurde.

Demgegenüber sind im Hinblick auf eine Differenzierung in Haupt- und Nebenerwerbslandwirte für die zweite Ausschreibungsrunde keine grundlegenden Unterschiede der Preiskalkulation und des Bieterverhaltens zu erkennen.

\subsection{Zusammenfassende Diskussion}

Die Konzeption und die Ergebnisse der beiden Ausschreibungsverfahren wurden jeweils hinsichtlich der Ausschreibungs- und der Befragungsergebnisse ausführlich betrachtet, so dass nachfolgend eine zusammenfassende Gegenüberstellung der wesentlichen Erkenntnisse erfolgen kann. 
Im Zuge dessen werden in Abschnitt 7.3.1 die Ausschreibungsergebnisse einbezogen und mit dem Ziel der Identifizierung ihrer Veränderung von der Ausschreibungsrunde 2004/2005 hin zur im Jahr 2006 durchgeführten Ausschreibung diskutiert. Im Anschluss thematisiert Abschnitt 7.3.2 die für beide Ausschreibungsverfahren relevanten Befragungsergebnisse ebenfalls mit dem Fokus auf entsprechenden Entwicklungen von der ersten hin zur zweiten Ausschreibungsrunde.

\subsubsection{Ausschreibungsergebnisse}

Nachdem sowohl die Ausschreibung 2004/2005 als auch die Ausschreibung im Jahr 2006 in den vorherigen Abschnitten insbesondere im Hinblick auf die Angebotspreise erörtert wurden, erfolgt nachfolgend eine Gegenüberstellung beider Ausschreibungsrunden unter Rückgriff auf einige zentrale Ergebnisse.

Hierzu werden sowohl die fristgerecht und formal korrekt eingegangenen Angebote als auch die angenommenen Angebote für alle Abstufungen der ökologischen Güter Grünland in die Betrachtung einbezogen. ${ }^{140}$

\subsubsection{Eingegangene Angebote}

Zunächst werden die fristgerecht und formal korrekt eingegangenen Angebote für die ökologischen Güter Grünland I, II und III in Tabelle 7-30 jeweils anhand der Spanne der Angebotspreise in $€$ pro Hektar sowie der auf ganze Euro gerundeten Mittelwerte der Angebotspreise, der Anzahl der angebotenen Schläge, der auf volle Hektar gerundeten Gesamtfläche und der Anzahl der ein Angebot abgebenden Landwirte gegenübergestellt und nachfolgend diskutiert.

\footnotetext{
${ }^{140}$ Eine Berücksichtigung der Auszahlungen an die Landwirte nach der Kontrolle erfolgt an dieser Stelle ausdrücklich nicht, da die notwendigen Ergebnisse lediglich für die erste Ausschreibung einbezogen werden können.
} 
Tab. 7-30: Gegenüberstellung zentraler Ergebnisse der eingegangenen Angebote für die Ausschreibungen 2004/2005 und 2006

\begin{tabular}{|l|c|c|}
\hline & $\begin{array}{c}\text { Ausschreibung } \\
2004 / 2005\end{array}$ & $\begin{array}{c}\text { Ausschreibung } \\
2006\end{array}$ \\
\hline Grünland I & & \\
\hline - Spanne der Angebotspreise in $€$ / ha & $40-250$ (Ø 101) & $25-160$ (Ø 94) \\
\hline - Anzahl der Schläge & 130 & 216 \\
\hline - Gesamtfläche in ha & 221 & 341 \\
\hline - Anzahl der Landwirte & 27 & 26 \\
\hline Grünland II & $55-300$ (Ø 142) & $75-300$ (Ø 148) \\
\hline - Spanne der Angebotspreise in $€$ / ha & 32 & 56 \\
\hline - Anzahl der Schläge & 53 & 83 \\
\hline - Gesamtfläche in ha & 16 & 18 \\
\hline - Anzahl der Landwirte & & 23 \\
\hline Grünland III & $100-350(\varnothing 203)$ & $150-450$ (Ø 257) \\
\hline - Spanne der Angebotspreise in $€$ / ha & 18 & 32 \\
\hline - Anzahl der Schläge & 37 & 7 \\
\hline - Gesamtfläche in ha & 8 & 23 \\
\hline - Anzahl der Landwirte & &
\end{tabular}

Quelle: Eigene Berechnungen.

Ein Vergleich der Preisspannen der eingegangenen Angebote zeigt, dass es sowohl in der Ausschreibung 2004/2005 als auch der wiederholten Ausschreibung im Jahr 2006 für alle Abstufungen der ökologischen Güter Grünland zu weitreichenden Unterschieden der individuellen Angebotspreise gekommen ist. Eine Ausweitung der Preisspanne hat sich im Vergleich der zweiten zu der ersten Ausschreibung einzig für die hochwertigsten Umweltleistungen eingestellt. Für die ökologischen Güter Grünland I ist es ebenso wie - in geringem Umfang - auch für die ökologischen Güter Grünland II zu einer Verringerung der Preisspanne gekommen wobei hier die Einflussmöglichkeiten durch die Wahl der Zuschlagsgrenze deutlich werden. Im Rahmen des ersten Ausschreibungsverfahrens wurde die Zuschlagsgrenze für die ökologischen Güter Grünland I auf $145 €$ pro Hektar festgelegt und demzufolge ist der Rückgang der Höchstgebote auf $160 €$ je Hektar in der zweiten Ausschreibungsrunde maßgeblich auf Anpassungsprozesse seitens der innerhalb der Ausschreibung 2004/2005 wegen eines zu hohen Angebotspreises abgelehnten Landwirte zurückzuführen (siehe auch Tabelle 7-31).

Für beide Ausschreibungen ist hinsichtlich des Preisniveaus festzustellen, dass dies mit einem höheren ökologischen Nutzen von Gut Grünland I über Gut Grünland II bis hin zu Gut Grünland III ebenfalls angestiegen ist. Ein Absinken des Preisniveaus 
der eingegangenen Angebote von der ersten zur zweiten Ausschreibung ist in einem moderaten Umfang lediglich für die ökologischen Güter Grünland I zu erkennen. Für die ökologischen Güter Grünland II hat sich diesbezüglich ein leichter und für die hochwertigsten ökologischen Güter Grünland III ein umfangreicher Anstieg der Angebotspreise eingestellt.

Im Hinblick auf die Anzahl der angebotenen Schläge ist es in der zweiten Ausschreibung für alle Qualitätsstufen der Umweltleistungen zu einem mitunter weitreichenden Anstieg gekommen. Im Zuge dessen hat sich für die ökologischen Güter Grünland I und II zudem die angebotene Gesamtfläche erhöht. Demgegenüber ist trotz einer größeren Zahl von Angeboten die gesamte angebotene Grünlandfläche für das Gut Grünland III zurückgegangen.

Im diesem Kontext ist erneut darauf zu verweisen, dass es bedingt durch die Beschränkung der Ausschreibung 2006 auf die bereits innerhalb der Ausschreibung 2004/2005 ein Angebot abgebenden Landwirte zu keiner Ausweitung des Kreises der potentiellen Anbieter gekommen ist. Folglich zeigt die Entwicklung von der ersten Ausschreibung hin zu der Ausschreibung 2006 in Form einer für alle ökologischen Güter steigenden Anzahl von Angeboten bei einer unveränderten Anzahl der teilnahmeberechtigten Landwirte sowohl ein zunehmendes Interesse als auch ein größeres Vertrauen seitens der Landwirte.

\subsubsection{Angenommene Angebote}

Neben den eingegangenen Angeboten und ihrer Entwicklung stellt sich zudem die zentrale Frage, über welche Angebote ein Vertrag geschlossen wurde und welche Veränderungen sich hinsichtlich dieser für den Finanzmitteleinsatz entscheidenden Angebote eingestellt haben. Hierzu werden die angenommenen Angebote für die ökologischen Güter Grünland I, II und III in Tabelle 7-31 jeweils anhand der Spanne der Angebotspreise in $€$ pro Hektar sowie der auf ganze Beträge gerundeten Mittelwerte der Angebotspreise, der Anzahl der angenommenen Schläge, der auf volle Hektar gerundeten Gesamtfläche und der Anzahl der einen Vertrag abschließenden Landwirte gegenübergestellt und anschließend diskutiert. 
Tab. 7-31: Gegenüberstellung zentraler Ergebnisse der angenommenen Angebote für die Ausschreibungen 2004/2005 und 2006

\begin{tabular}{|l|c|c|}
\hline & $\begin{array}{c}\text { Ausschreibung } \\
2004 / 2005\end{array}$ & $\begin{array}{c}\text { Ausschreibung } \\
2006\end{array}$ \\
\hline Grünland I & & \\
\hline - Spanne der Angebotspreise in $€$ / ha & $40-145$ (Ø 85) & $25-90$ (Ø 67) \\
\hline - Anzahl der Schläge & 109 & 89 \\
\hline - Gesamtfläche in ha & 198 & 130 \\
\hline - Anzahl der Landwirte & 20 & 10 \\
\hline Grünland II & & $75-200$ (Ø 138) \\
\hline - Spanne der Angebotspreise in $€$ / ha & $55-300$ (Ø 142) & 52 \\
\hline - Anzahl der Schläge & 32 & 77 \\
\hline - Gesamtfläche in ha & 53 & 17 \\
\hline - Anzahl der Landwirte & 16 & 23 \\
\hline Grünland III & $100-350(\varnothing 203)$ & $150-450$ (Ø 257) \\
\hline - Spanne der Angebotspreise in $€$ / ha & 18 & 32 \\
\hline - Anzahl der Schläge & 37 & 7 \\
\hline - Gesamtfläche in ha & 8 & 75 \\
\hline - Anzahl der Landwirte & &
\end{tabular}

Quelle: Eigene Berechnungen.

Den Ausführungen vorangestellt wird in diesem Zusammenhang die Höhe des jeweils zur Verfügung stehenden Ausschreibungsbudgets sowie die seitens des Regionalen Beirats Northeim mit dem Nachfragekatalog beschlossene und im Rahmen der Zuschlagserteilung zu berücksichtigende Aufteilung auf die drei Abstufungen der Umweltleistungen.

So stand für die Ausschreibung 2004/2005 ein Budget von $30.000 €$ zur Verfügung und es wurde beschlossen, davon zwei Drittel (20.000 €) für die ökologischen Güter Grünland I und jeweils ein Sechstel (5.000 €) für die ökologischen Güter Grünland II und III einzusetzen, wobei je nach Qualität der angebotenen Umweltleistungen grundsätzlich die Option einer Verlagerung hin zu den höherwertigen ökologischen Gütern eingeräumt wurde. Für die Ausschreibungsrunde 2006 stand ein Budget im Umfang von $26.000 €$ zur Verfügung und hinsichtlich der Budgetaufteilung wurde eine Anpassung dahingehend vorgenommen, alle Qualitätsstufen gleichberechtigt im Umfang von einem Drittel des Budgets bzw. rund $8.667 €$ zu berücksichtigen.

Die Preisspannen der angenommenen Angebote verdeutlichen für beide Ausschreibungsrunden und alle Abstufungen der ökologischen Güter eine Verschiedenartigkeit der Angebotspreise und im Zuge dessen die bei der Zuschlagserteilung vollzogene Vertragsdifferenzierung für jeweils identische 
Umweltleistungen. Demzufolge konnte die Erfüllung der praktischen Ziele von Ausschreibungsverfahren und im Zuge dessen ihre potentielle Vorteilhaftigkeit gegenüber Einheitsprämien ausdrücklich auch innerhalb einer wiederholten Ausschreibung gezeigt werden.

Die Betrachtung des Preisniveaus weist für die angenommenen Angebote ebenfalls einen mit zunehmenden Anforderungen an die pflanzliche Biodiversität einhergehenden Anstieg der Angebotspreise für beide Ausschreibungen aus. Ein Vergleich der Ausschreibung 2004/2005 und der Ausschreibung 2006 zeigt für die ökologischen Güter Grünland I und II einen Rückgang des Preisniveaus der angenommenen Angebote. Demgegenüber ist das Preisniveau für die ökologischen Güter Grünland III angestiegen, was maßgeblich in der Anpassung des Nachfragekatalogs und der darin vollzogenen relativen Verschiebung der Nachfrage hin zu den höherwertigen Umweltleistungen begründet ist da innerhalb des Forschungsprojekts der Ansatz verfolgt wurde, dass gesamte zur Verfügung stehende Budget einzusetzen und insbesondere eine möglichst große Anzahl ökologisch hochwertigster Flächen vertraglich zu binden. Zweifelsohne hätte an dieser Stelle auch ein Reservationspreis beispielsweise in Höhe von $350 €$ pro Hektar gesetzt und im Zuge dessen ein Preisanstieg für die hochwertigsten Umweltleistungen verhindern werden können.

Hinsichtlich der Anzahl der angenommenen Schläge offenbaren sich ebenso die Auswirkungen der von der ersten zur zweiten Ausschreibung vorgenommenen Anpassung der Budgetverteilung. Dies führte dazu, dass innerhalb der relativ geringwertigsten ökologischen Güter Grünland I sowohl weniger Schläge als auch eine deutlich geringere Fläche vertraglich gebunden wurden. Im Gegensatz dazu konnten innerhalb der ökologischen Güter Grünland II im Jahr 2006 eine höhere Anzahl von Angeboten und eine größere Fläche angenommen werden. Für die ökologischen Güter Grünland III wurde in der zweiten Ausschreibungsrunde insgesamt eine höhere Anzahl kleinerer Schläge angeboten, so dass die Gesamtfläche hier zurückgegangen ist. 


\subsubsection{Befragungsergebnisse}

Im Hinblick auf die Betrachtung der Preiskalkulation und des Bieterverhaltens der Landwirte werden thematisch mit der ausschreibungsrelevanten Kommunikation und der Einbeziehung über die Produktionskosten hinausgehender Aspekte im Rahmen der Kalkulation des Angebotspreises zwei grundlegende Bereiche unterschieden.

I. Hinsichtlich der ausschreibungsrelevanten Kommunikation der Landwirte in der Modellregion zeigt sich für beide Ausschreibungsverfahren ein sehr einheitliches Bild. Eine Beratung mit anderen Landwirten haben in der ersten Ausschreibungsrunde 20,8 \% und in der zweiten Ausschreibung 12,0 \% der an den Befragungen teilnehmenden Landwirte vorgenommen. Demzufolge ist der grundsätzliche Kommunikations- und Beratungsbedarf für beide Ausschreibungen als gering einzuschätzen und im Zuge der zweiten Ausschreibung zudem leicht zurückgegangen.

Dies spricht sowohl für eine steigende Vertrautheit der Landwirte im Umgang mit dem neuartigen Honorierungsinstrument als auch maßgeblich gegen eine Gefahr der zunehmenden ausschreibungsrelevanten Kommunikation bei wiederholten Ausschreibungen.

Dem Aspekt von Preisabsprachen der Landwirte wurde sowohl durch eine Frage zu aktuell vollzogenen Preisabsprachen als auch perspektivisch hinsichtlich der Einschätzung der Relevanz zukünftiger Preisabsprachen nachgegangen.

Diesbezüglich ist zu erkennen, dass lediglich ein Landwirt innerhalb der zweiten Ausschreibung angegeben hat, eine Preisabsprache getroffen zu haben und es ist zu resümieren, dass es in beiden Ausschreibungsrunden zu keinen bedeutsamen und den praktischen Vorteilen einer Ausschreibung entgegenwirkenden Preisabsprachen gekommen ist. Eine Einschätzung, die ganz deutlich ebenfalls durch die Ergebnisse der Angebotspreise unterstützt wird.

Die Frage nach einer Beurteilung der zukünftigen Relevanz von Preisabsprachen hat mit 41,7 \% in der ersten Befragung und mit 40,0 \% in der zweiten Befragung jeweils weniger als die Hälfte der Landwirte positiv beantwortet. Demzufolge sind auf der Grundlage der Befragungsergebnisse grundsätzliche Tendenzen für zukünftige Preisabsprachen zu erkennen, wobei es als unsicher anzusehen ist, inwieweit sich diese Überlegungen in den Handlungen der Landwirte widerspiegeln werden. 
Diesbezüglich zeigen die Ergebnisse der Ausschreibung 2006, dass dort die im Rahmen der ersten Befragung grundsätzlich zu erkennende Bereitschaft Preisabsprachen zu treffen, faktisch keine Berücksichtigung mehr gefunden hat und dies eine Relativierung des im Zuge der Befragungsergebnisse zu erkennenden zukünftigen Einflusses von Preisabsprachen notwendig macht.

Den Bereich der ausschreibungsrelevanten Kommunikation und der potentiellen Einbeziehung exogener Vorgaben in die individuellen Angebotspreise abschließend wird die Frage der Inanspruchnahme und des Einflusses von Beratungsinstitutionen im Zuge der Angebotserstellung aufgegriffen.

Die Befragungsergebnisse lassen erkennen, dass im Rahmen der ersten Ausschreibung ein Viertel der an der Befragung teilnehmenden Landwirte eine Beratung in Anspruch genommen und dabei Anhaltspunkte oder Vorgaben für ihre Angebotspreise bekommen hat. Von diesen sechs Landwirten haben vier Landwirte die Vorgaben letztlich auch bei der Ermittlung ihres Angebotspreises berücksichtigt. Für das zweite Ausschreibungsverfahren zeigt sich ein deutlicher Rückgang des Beratungsbedarfs und nunmehr haben lediglich 8,0 \% der Landwirte eine Beratung genutzt. Dabei hat ein Landwirt Anhaltspunkte für Angebotspreise erhalten, wobei sie keine Berücksichtigung gefunden haben. Demzufolge ist festzuhalten, dass sowohl der Beratungsbedarf als auch die Möglichkeiten einer Einflussnahme seitens der Beratungsinstitutionen von der ersten zur zweiten Ausschreibung deutlich zurückgegangen sind und hier ebenfalls keine sich negativ auf die Ausschreibungsverfahren auswirkenden Effekte zu erkennen sind.

II. Der zweite thematische Komplex behandelt die Frage, in welchem Umfang bei der Preiskalkulation jeweils über die Produktionskosten hinausgehende Aspekte in Form des Zeitaufwands der Angebotserstellung und eines Gewinnzuschlags einbezogen wurden.

Eine Kalkulation und Berücksichtigung des Zeitaufwands bzw. der Transaktionskosten wurde in beiden Ausschreibungsrunden von weniger als der Hälfte der Landwirte vorgenommen. In der Ausschreibung 2004/2005 haben 45,8 \% angeführt den Zeitaufwand entsprechend einkalkuliert zu haben und in der Ausschreibung 2006 haben dies 48,0 \% angegeben. 
Eine differenzierte Betrachtung hinsichtlich der Bewirtschaftungs- und Betriebsform hat für die erste Ausschreibung bedingt durch den mit 16,7 \% sehr geringen Anteil der ihren Zeitaufwand berücksichtigenden Nebenerwerbslandwirte die Vermutung aufkommen lassen, dass Nebenerwerbslandwirte aufgrund ihrer nicht ausschließlichen Abhängigkeit der durch die landwirtschaftliche Produktion erzielten Einnahmen geringere Angebotspreise abgeben und damit die Wahrscheinlichkeit der Zuschlagserteilung innerhalb eines Ausschreibungsverfahrens erhöhen können. Diese Einschätzung hat sich in der zweiten Ausschreibung jedoch nicht bestätigt und kann nicht weiter aufrechterhalten werden.

Einen über die Kostendeckung hinausgehenden Gewinnzuschlag hat in der Ausschreibung 2004/2005 mit 26,1 \% und in der Ausschreibung 2006 mit 20,0 \% jeweils ein geringer Anteil der Landwirte einbezogen, wobei hinsichtlich der Bewirtschaftungs- und Betriebsform keine grundlegend abweichenden Tendenzen zu erkennen sind. Demzufolge zeigt sich bei der Berücksichtigung eines Gewinnzuschlags ebenfalls die grundsätzlich für beide Ausschreibungsrunden einheitliche geringe Relevanz. Die Höhe des einkalkulierten Gewinnzuschlags hingegen hat sich von der ersten zur zweiten Ausschreibung verändert und ist im Mittel von 21,7 \% auf 11,2 \% zurückgegangen.

Zusammenfassend lässt der noch sehr geringe Anteil von Landwirten, der in beiden Ausschreibungen einen Gewinnzuschlag in die Kalkulation einbezogen hat, ebenso wie der von weniger als der Hälfte der Landwirte einkalkulierte Zeitaufwand der Angebotserstellung ähnliche Interpretationen $\mathrm{zu}$.

So ist zum einen zu vermuten, dass noch nicht bei allen landwirtschaftlichen Betriebsleitern das unternehmerische Kalkulieren speziell hinsichtlich einer über die Produktionskosten hinausgehenden Kostendeckung und einer Gewinnerzielung ausgeprägt ist. Dies ist sehr wahrscheinlich darin begründet, dass Prämienzahlungen in Agrarumweltprogrammen bislang lediglich als Kompensationszahlungen eingesetzt werden, was einer umfassenden und eigenverantwortlichen Preiskalkulation entgegensteht. Zum anderen ist die Unsicherheit der Zuschlagsgrenze und des für die Ausschreibung zur Verfügung stehenden Budgets für den Landwirt relevant, da mit der Erhöhung des Angebotspreises in der Regel die Wahrscheinlichkeit einer Zuschlagserteilung abnimmt. 
Aus Sicht der Agrarverwaltung und im Hinblick auf einen effizienten öffentlichen Mitteleinsatz ist ein durch die Berücksichtigung über die Produktionskosten hinausgehender Aspekte steigender Angebotspreis zweifelsohne kritisch zu sehen. Andererseits ist zu erwarten, dass sich mit einer höheren Auszahlungssumme die Akzeptanz von Agrarumweltmaßnahmen erhöht, was wiederum auch seitens der Agrarverwaltung wünschenswert ist und die Auswahl der angebotenen Flächen im Rahmen eines zukünftigen Ausschreibungsverfahrens vergrößert.

Hier muss in der praktischen Umsetzung letztlich eine politische Abwägung in Form des Ausschreibungsdesigns und der Wahl der Zuschlagsgrenzen bzw. der Setzung eines Reservationspreises erfolgen. Dies verdeutlicht erneut sowohl die Flexibilität und die damit verbundenen Handlungsalternativen als auch die Verantwortung der Agrarverwaltung bei der Nutzung eines Ausschreibungsverfahrens für Umweltleistungen. 


\section{Die Ermittlung und Bewertung der Transaktionskosten}

Im vorherigen Kapitel wurden mit den Angebotspreisen für ökologische Güter auf Grünlandflächen die Ergebnisse der Ausschreibungsrunden 2004/2005 und 2006 betrachtet. Darüber hinaus wurde mit der Untersuchung der Preiskalkulation und des Bieterverhaltens der Frage nachgegangen, wie diese Angebotspreise zustande gekommen sind.

Vervollständigt werden die empirischen Untersuchungen im Nachfolgenden mit der Ermittlung und Bewertung der für die Landwirte im Rahmen der Angebotsabgabe in beiden Ausschreibungsrunden relevanten Transaktionskosten.

Hierzu wird in Abschnitt 8.1 einführend auf grundlegende Aspekte zur Ermittlung und Bewertung von Transaktionskosten eingegangen und der Stand der Forschung umrissen. Die Definition der Transaktionskosten und das hier gewählte methodische Vorgehen werden in Abschnitt 8.2 erläutert.

Hinsichtlich der Ermittlung der Transaktionskosten wird zwischen dem Ausschreibungsverfahren im Zeitraum 2004/2005 und der Ausschreibung im Jahr 2006 unterschieden.

Die Transaktionskosten des ersten Ausschreibungsverfahrens werden auf der Grundlage von zwei empirischen Arbeiten berechnet, wobei die erste Befragung in Abschnitt 8.3 thematisiert wird. Auf die zweite und hinsichtlich der Transaktionskosten der Landwirte weniger umfangreiche Erhebung wird in Abschnitt 8.4 eingegangen. In Abschnitt 8.5 werden die vor dem Hintergrund der Ermittlung und Bewertung der Transaktionskosten in der Ausschreibung 2006 relevanten und in der dritten Befragung ermittelten Ergebnisse behandelt.

In einer Zusammenführung der Ergebnisse und unter Einbeziehung weiterer Daten erfolgt in Abschnitt 8.6 die monetäre Bewertung des ermittelten Zeitaufwands und somit die Berechnung der Transaktionskosten.

Das Kapitel abschließend werden die Transaktionskosten der Landwirte in Abschnitt 8.7 zusammenfassend zur Bewertung des Ausschreibungsverfahrens herangezogen. Darüber hinausgehend wird der eingangs angesprochene Artikel 39 der ELERVerordnung aufgegriffen und die Transaktionskosten dahingehend diskutiert, ob sie als Bewertungsmaßstab für Zahlungen an Landwirte dienen können. 


\subsection{Grundlagen}

Trotz der mittlerweile umfangreichen Forschungsarbeit zur Transaktionskostenökonomik ${ }^{141}$ hat sich keine eindeutige Definition herausgebildet, was unter Transaktionskosten zu verstehen ist und wie sie zu messen sind. So wird bislang vor allem der heuristische Wert von Transaktionskosten erörtert und ihre ökonomische Relevanz betont, jedoch oftmals ohne der Diskussion eine Ermittlung der betrachteten Transaktionskosten voranzustellen, was insbesondere der mangelnden Verfügbarkeit notwendiger Daten und der hohen (Transaktions-)Kosten empirischer Arbeiten zur Datenermittlung geschuldet ist. ${ }^{142}$ Infolgedessen erscheint es notwendig, sowohl das theoretische Konzept als auch die praktische Relevanz der Transaktionskostenökonomik zu hinterfragen und ihre empirische Basis zu erweitern.

Als bisherige Ansätze der Berücksichtigung von Transaktionskosten im Kontext von Agrarumweltprogrammen sind vor allem die folgenden drei Arbeiten von Falconer und Whitby (1999), Vatn (2001) und der OECD (2001b) von Interesse.

I. Falconer und Whitby haben innerhalb des „Environmental Sensitive Areas“ (ESAs) Programms in Großbritannien eine Analyse durchgeführt, im Rahmen derer die administrativen Transaktionskosten als ein Teil der gesamten Transaktionskosten evaluiert wurden. Ziel der Untersuchung war es, die relevanten Transaktionskosten abzuschätzen und Einsparpotentiale aufzuzeigen (Falconer und Whitby 1999, S. 1-4). Vor dem Hintergrund des Effizienzziels betonen Falconer und Whitby die praktische Bedeutung der unterschiedlichen Transaktionskosten verschiedener politischer Maßnahmen und im Zuge dessen die Notwendigkeit der Wahl praktischer institutioneller Ausgestaltungen unter Berücksichtigung der dadurch zu erwartenden gesamten Kosten (Falconer und Whitby 1999, S. 6-7).

Einführend werden hierzu für die beiden Akteure Verwaltung und Landwirte eine grundlegende Unterscheidung in fixe und variable Transaktionskosten vorgenommen sowie die drei Kategorien Information, Vertragsabschluss und Durchführung unterschieden (Falconer und Whitby 1999, S. 12). Letztlich liegt der Fokus vor allem aus Gründen der Datenverfügbarkeit auf den staatlichen Transaktionskosten. Die

\footnotetext{
141 Für eine umfassende Darstellung des Konzepts der Transaktionskostenökonomik und weitere grundlegende Definitionen und Abgrenzungen siehe Kapitel Vier dieser Arbeit.

${ }^{142}$ Einen Überblick geben diesbezüglich exemplarisch Allen (1991; 2000) und Wang (2003).
} 
große Bedeutung der seitens der Landwirte relevanten Transaktionskosten wird jedoch ausdrücklich betont und ihre Untersuchung als die zentrale zukünftige Forschungsarbeit angesehen, um eine umfassende Bewertung von Agrarumweltmaßnahmen zu ermöglichen. Darüber hinaus sehen Falconer und Whitby weiteren Forschungsbedarf in der Gegenüberstellung der administrativen Kosten und des ökologischen Nutzens in Form einer Kosten-Nutzen-Betrachtung (Falconer und Whitby 1999, S. 26-27).

Zusammenfassend verdeutlicht die Arbeit die praktische Relevanz administrativer Transaktionskosten und als zentrales Ergebnis kommt sie zu der Schlussfolgerung, dass sich mit einer zunehmenden Vertragslaufzeit transaktionskostenmindernde Effekte eingestellt haben. Dies legt nahe, erfolgreiche Verträge so lange wie möglich aufrecht zu erhalten bzw. zu verlängern und Landwirten unter Berücksichtigung erfolgter transaktionsspezifischer Investitionen Anreize zu geben, ihre in einem Förderprogramm berücksichtigten Flächen langfristig entsprechend zu bewirtschaften (Falconer und Whitby 1999, S. 61-62).

II. Der Einfluss von Transaktionskosten auf politische Entscheidungen im Kontext der Multifunktionalität und dabei vor allem der Trade-off zwischen Transaktionskosten und Zielgenauigkeit politischer Maßnahmen unter Einbeziehung verschiedener ökonomischer Güter ist Gegenstand der Arbeit von Vatn (Vatn 2001, S. 1-5).

Hierbei betont Vatn grundsätzlich die Notwendigkeit der praktischen Berücksichtigung und den Einfluss von Transaktionskosten, wobei das Problem einer asymmetrischen Informationsverteilung zwischen den handelnden Akteuren und der darin bedingten Unkenntnis der mit einer politischen Maßnahme verbundenen Transaktionskosten aufgezeigt wird (Vatn 2001, S. 5-9).

Mit Blick auf die abzuleitenden politischen Handlungsempfehlungen verfolgt Vatn den Ansatz, die Transaktionskosten und die Charakteristika der betrachteten Güter zu kombinieren und kommt zu dem Schluss, dass bei einem direkten Zusammenhang der Produktion privater Güter und relevanter öffentlicher Güter eine politische Steuerung der Menge der bereitgestellten öffentlichen Güter zu geringen Transaktionskosten durch Preiseingriffe bei den privaten Gütern erfolgen kann. Für den Fall, dass die Produktion privater Güter keinen oder einen negativen Einfluss auf die Bereitstellung der erwünschten öffentlichen Güter ausübt, ist ein direkter 
staatlicher Eingriff notwendig, der durch bestehende Informationsasymmetrien mit hohen politischen Transaktionskosten der Zielfindung und Durchsetzung der Maßnahme verbunden ist (Vatn 2001, S. 17).

Zur Lösung der Problematik hoher politischer Transaktionskosten sieht Vatn einerseits die Verfolgung eines integrierten Ansatzes der Produktion privater und öffentlicher Güter und dort wo dies nicht möglich ist, die Notwendigkeit marktähnliche Strukturen auch für öffentliche Güter zu implementieren und im Zuge dessen die politischen Transaktionskosten zu reduzieren und die Zielgenauigkeit der Agrarumweltpolitik zu erhöhen (Vatn 2001, S. 17-18).

III. Eine zusammenfassende Arbeit der OECD berücksichtigt Transaktionskosten ebenfalls im Zusammenhang mit Fragen der Multifunktionalität und argumentiert, dass die fehlende Berücksichtigung von Transaktionskosten in der Praxis zur suboptimalen Umsetzung und Ausgestaltung politischer Maßnahmen führen kann (OECD 2001b, S. 2-3).

Dabei unterscheidet die OECD Transaktionskosten danach, inwiefern sie mit der Implementierung und Durchführung einer politischen Maßnahme in Verbindung stehen und bildet die beiden Kategorien „,non policy-related transaction costs“ und „policy-related transaction costs“ (OECD 2001b, S. 3).

Die in der vorliegenden Arbeit relevanten Transaktionskosten der Landwirte werden von der OECD allgemein als Informationskosten der Entscheidung über eine Teilnahme oder Nichtteilnahme an einer angebotenen Maßnahme definiert und den „policy-related transaction costs“ zugerechnet (OECD 2001b, S. 3-4). Ohne weitere Abgrenzungen vorzunehmen oder ein praktisches Vorgehen aufzuzeigen, wird seitens der $O E C D$ zudem ausdrücklich die hohe Relevanz der Transaktionskosten der Landwirte betont und sowohl die Notwendigkeit ihrer praktischen Ermittlung als auch ihre Einbeziehung in Kosten-Nutzen-Analysen zur Bewertung politischer Maßnahmen hervorgehoben (OECD 2001b, S. 4-5).

Des Weiteren ist in diesem Kontext auf Arbeiten von Colby (1990), Smith und Tomasl (1995), Allen und Lueck (1996), McCann und Easter (1999), Falconer et al. (2001) und Vatn (2002) hinzuweisen, welche ebenfalls die mit der Implementierung von agrarpolitischen Maßnahmen verbundenen Transaktionskosten untersucht haben; dies jedoch ebenfalls ohne auf die Transaktionskosten der Landwirte Bezug zu 
nehmen. Zudem gibt Wang (2003) einen Überblick bisheriger Arbeiten zur Messung von Transaktionskosten in verschiedenen thematischen Kontexten.

Wie aus der Darstellung bisheriger Arbeiten hervorgegangen ist, lag der Fokus zum großen Teil auf Überlegungen des Einflusses von Transaktionskosten auf politische Maßnahmen und der Bewertung administrativer Transaktionskosten.

Die Transaktionskosten der Landwirte haben nur in geringem Maß Berücksichtigung gefunden und eine Ermittlung entsprechend des hier verfolgten Ansatzes hat bislang noch nicht stattgefunden, so dass in Abgrenzung $\mathrm{zu}$ den bisherigen Arbeiten nachfolgend die Quantifizierung der für die Landwirte im Zuge der Angebotsabgabe in zwei Ausschreibungsverfahren anfallenden Transaktionskosten im Mittelpunkt steht.

\subsection{Definition und methodisches Vorgehen}

Die innerhalb der vorliegenden Arbeit thematisierten Transaktionskosten beziehen sich auf die Seite der Landwirte, wobei zudem eine Beschränkung auf die privaten Transaktionskosten vollzogen wird, welche für alle Landwirte relevant sind und demzufolge ausschließlich die Ex-ante-Transaktionskosten bis zum Zeitpunkt der Angebotsabgabe betrachtet werden. ${ }^{143}$ Nach Ende der Angebotsfrist sind seitens der Landwirte keine Veränderungen ihres Angebots mehr möglich und alle weiteren Kosten zur Erbringung der ökologischen Leistung sind hier als Produktionskosten von den Transaktionskosten abzugrenzen.

Infolgedessen wird der bewertete Zeitaufwand für die Anforderung der Ausschreibungsunterlagen, die Informationsbeschaffung, die Bestimmung der anzubietenden ökologischen Güter, die Auswahl der zugehörigen Schläge, die Preiskalkulation und das Ausfüllen der Ausschreibungsunterlagen als Transaktionskosten definiert. Hierbei sind die mit der Anforderung der Ausschreibungsunterlagen und entsprechender Informationen verbundenen Kosten für die Landwirte als fixe Transaktionskosten anzusehen, da sie je Ausschreibungsrunde einmalig anfallen und unabhängig von der Anzahl der

\footnotetext{
143 Die im Zuge eines Ausschreibungsverfahrens relevanten administrativen staatlichen Transaktionskosten werden nicht betrachtet. Dies ist darin begründet, dass innerhalb des Forschungsprojekts noch keine operative Einbindung der Agrarverwaltung in den Ablauf des Ausschreibungsverfahrens stattgefunden hat und folglich entsprechende Daten im Verlauf dieser Projektphase noch nicht erhoben werden konnten.
} 
angebotenen Schläge sind. Demgegenüber sind alle weiteren im Verlauf der Angebotserstellung auftretenden Transaktionskosten als variable Transaktionskosten zu betrachten, da sie in ihrer Höhe durch die Anzahl der Angebote eines Landwirts beeinflusst werden.

$\underline{\text { Für die Transaktionskostenermittlung relevante Arbeitsschritte: }}$

1. Anforderung der Ausschreibungsunterlagen

2. Informationsbeschaffung

3. Angebotsermittlung

4. Ausfüllen der Ausschreibungsunterlagen

Das methodische Vorgehen zur Ermittlung der Transaktionskosten ist wie folgt gewählt.

Der obigen Definition entsprechend wird durch drei schriftliche Befragungen zuerst der für die Angebotsabgabe benötigte Zeitaufwand der Landwirte ermittelt. Von diesen Befragungen beziehen sich zwei auf die erste und eine auf die zweite Ausschreibungsrunde. Die am Ausschreibungsverfahren teilnehmenden Landwirte wurden dabei jeweils gebeten, den von ihnen zur Angebotserstellung benötigten Zeitaufwand entsprechend in den Fragebögen anzugeben. Dieser Zeitaufwand stellt die Grundlage der Transaktionskostenermittlung dar und wird in einem zweiten Schritt monetär bewertet.

Die monetäre Bewertung erfolgt in der Form, dass der ermittelte Zeitaufwand mit einem Stundenlohn in Verbindung gesetzt wird und sich daraus die relevanten Transaktionskosten ergeben. Doch welcher Stundenlohn sollte hierfür herangezogen werden? Im Rahmen der vorliegenden Arbeit wird der Ansatz verfolgt, die Landwirte selbst nach einer Einschätzung des von ihnen für die Angebotsabgabe als angemessen empfundenen Stundenlohns zu fragen und folglich ihre subjektive Bewertung in die Berechnung der Transaktionskosten einzubeziehen. 


\subsection{Die erste Befragung}

Im Folgenden werden einführend in einer kurzen Übersicht sowohl die Konzeption der ersten Befragung als auch die teilnehmenden Landwirte skizziert und im Anschluss die ermittelten Ergebnisse zum Zeitaufwand der Angebotserstellung präsentiert.

\subsubsection{Konzeption und teilnehmende Landwirte}

Für die Befragung wurden im Zeitraum Juni bis Juli 2004 jene 140 Landwirte angeschrieben, die durch die Anforderung von Ausschreibungsunterlagen ein grundsätzliches Interesse an der Ausschreibung bekundet haben.

Ihnen wurde zusammen mit den Ausschreibungsunterlagen in einem gesonderten Umschlag ein Fragebogen zugeschickt, der neben den Fragen zum Zeitaufwand ebenfalls Aspekte zur Teilnahme am Ausschreibungsverfahren, zum Konzept der ergebnisorientierten Honorierung ökologischer Leistungen der Landwirtschaft, zu den bestehenden Agrarumweltprogrammen und betrieblichen Daten evaluieren sollte. $^{144}$

Von den 140 am Ausschreibungsverfahren interessierten Landwirten haben 35 an der Befragung teilgenommen, wovon wiederum 30 Landwirte in der Ausschreibungsrunde 2004/2005 ein Angebot abgegeben haben. Diese 30 Landwirte sind für die weiteren Betrachtungen entscheidend, da nur von den auch am Ausschreibungsverfahren teilnehmenden Landwirten entsprechende Angaben zu dem von ihnen benötigten Zeitaufwand zu erwarten sind. Insgesamt haben 38 Landwirte innerhalb der ersten Ausschreibung ein formal korrektes und fristgerechtes Angebot abgegeben und demzufolge sind 78,9 \% der relevanten Landwirte auch in der ersten Befragung repräsentiert. $^{145}$

$\mathrm{Zu}$ den teilnehmenden 30 Landwirten ist darüber hinaus anzumerken, dass 20 Landwirte eine konventionelle und zehn eine ökologische Landwirtschaft betreiben. Zwölf Landwirte bewirtschaften ihren Betrieb im Haupt- und 18 im Nebenerwerb, wobei in dem hier zugrunde liegenden Kontext zudem von Interesse ist, dass bislang

\footnotetext{
${ }^{144}$ Der Fragebogen findet sich im Anhang 3 der Arbeit.

${ }^{145}$ Die im Folgenden präsentierten Ergebnisse beziehen sich, soweit keine anderen Angaben gemacht werden, auf diese Landwirte.
} 
17 der 30 Landwirte bereits mindestens einmal an Agrarumweltprogrammen teilgenommen haben, was einem Anteil von 56,67 \% entspricht.

\subsubsection{Ergebnisse}

Die in Abschnitt $8.6 \mathrm{zu}$ berechnenden Transaktionskosten beziehen sich für die Ausschreibung 2004/2005 insbesondere auf den innerhalb dieser ersten schriftlichen Befragung ermittelten Zeitaufwand.

Die relevanten Ergebnisse werden nachfolgend jeweils anhand des minimalen und des maximalen Werts, des Mittelwerts, des Medians und der Standardabweichung präsentiert. Vorangestellt ist den Ergebnissen als Zitat jeweils die entsprechende Frage in der Form, wie sie im Fragebogen gestellt war.

„Wie viel Zeit haben Sie ungefähr für die Beschaffung der Ausschreibungsunterlagen benötigt?“

Diese Frage bezieht sich auf den ersten Arbeitsschritt im Verlauf der Angebotsabgabe. Hierbei haben 27 Landwirte eine Angabe gemacht und der Zeitaufwand umfasst eine Spanne von 2 Minuten bis 200 Minuten. Der Mittelwert liegt bei 31,33 Minuten und der Median bei 15 Minuten, was nicht zuletzt in Verbindung mit einer Standardabweichung von 47,36 Minuten eine große Differenz des Zeitaufwands zeigt. Tabelle 8-1 gibt eine Übersicht über den Zeitaufwand der Beschaffung der Ausschreibungsunterlagen.

Tab. 8-1: Zeitaufwand - Beschaffung der Ausschreibungsunterlagen $(2004 / 2005)$

\begin{tabular}{|l|c|c|c|c|c|}
\hline $\mathrm{n}=27$ & Min. & Max. & Mittelwert & Median & Std.abw. \\
\hline Minuten & 2 & 200 & 31,33 & 15 & 47,36 \\
\hline
\end{tabular}

Quelle: Eigene Berechnungen.

Die große Spanne ist maßgeblich darin zu begründen, dass einige Landwirte die Ausschreibungsunterlagen lediglich mit einem kurzen Telefonanruf oder per E-Mail angefordert haben und sich folglich ein zu vernachlässigender oder sehr geringer Zeitaufwand ergeben hat. Demgegenüber haben einige Landwirte eine im Einzelfall sehr zeitaufwändige Fahrt zu einer Informationsveranstaltung auf sich genommen um die Ausschreibungsunterlagen zu beschaffen. Diese Verteilung wird in Abbildung 8- 
1 durch die Darstellung der einzelnen Zeitangaben und der Häufigkeit ihrer Nennung verdeutlicht.

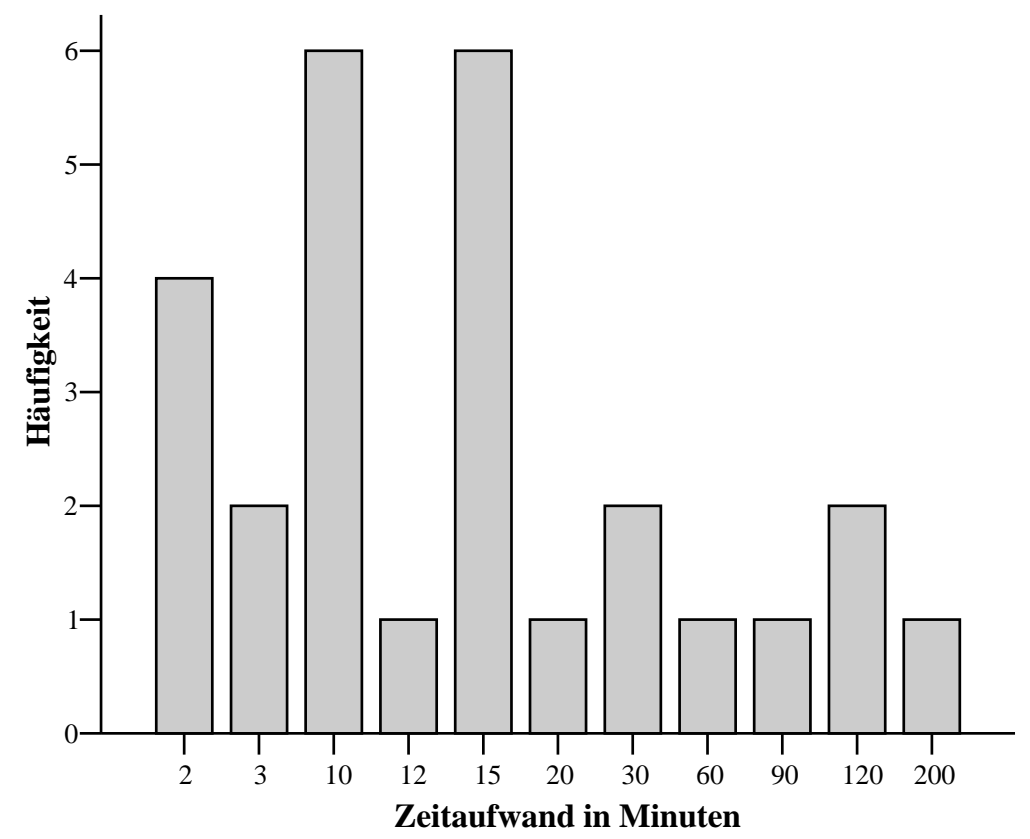

Abb. 8-1: Zeitaufwand zur Beschaffung der Ausschreibungsunterlagen in Minuten und Häufigkeit der Nennung (2004/2005)

Quelle: Eigene Darstellung nach eigenen Berechnungen.

Sowohl die Ergebnisse in Tabelle 8-1 als auch die grafische Darstellung zeigen bereits hinsichtlich der Beschaffung der Ausschreibungsunterlagen eine große Heterogenität des benötigten Zeitaufwands.

„Wie lange haben Sie ungefähr für das Lesen und Verstehen der gesamten Ausschreibungsunterlagen (ohne Kalkulation des Angebotspreises und Ausfüllen der Ausschreibungsunterlagen) benötigt?“

Die zweite Frage bezieht sich auf den für das Lesen und grundsätzliche Verstehen der Ausschreibungsunterlagen notwendigen Zeitbedarf. Diese Abgrenzung hat Beachtung verdient, da es sich bei der durchgeführten Ausschreibung um ein für Landwirte grundlegend neues Verfahren mit entsprechend neuen Unterlagen und Anforderungen handelt.

Der benötigte Zeitaufwand wurde von 29 Landwirten im Mittel mit 39,48 Minuten benannt und umfasst mit einer Standardabweichung von 21,93 Minuten und einem Median von 30 Minuten eine Spanne von 10 Minuten bis 100 Minuten. Die Ergebnisse sind in Tabelle 8-2 im Überblick dargestellt. 
Tab. 8-2: Zeitaufwand - Lesen und Verstehen der Ausschreibungsunterlagen $(2004 / 2005)$

\begin{tabular}{|l|c|c|c|c|c|}
\hline $\mathrm{n}=29$ & Min. & Max. & Mittelwert & Median & Std.abw. \\
\hline Minuten & 10 & 100 & 39,48 & 30 & 21,93 \\
\hline
\end{tabular}

Quelle: Eigene Berechnungen.

Zur Veranschaulichung dieser Ergebnisse zeigt Abbildung 8-2 zudem die individuellen Angaben der Landwirte.

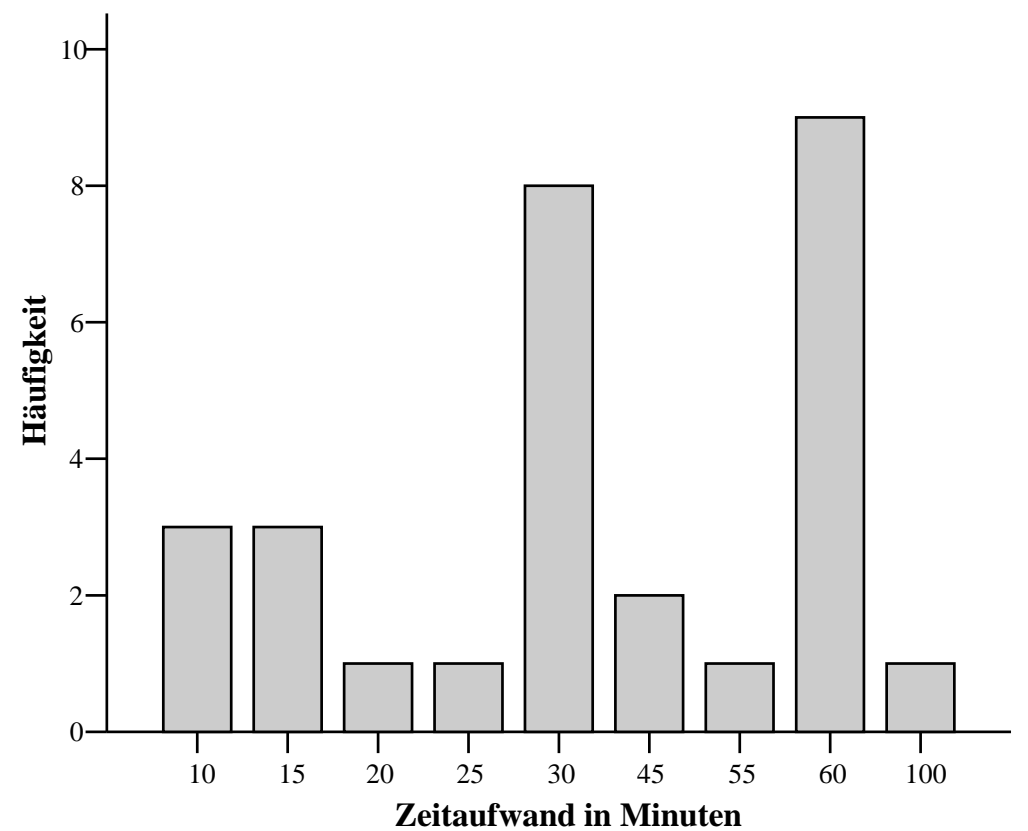

Abb. 8-2: Zeitaufwand zum Lesen und Verstehen der Ausschreibungsunterlagen in Minuten und Häufigkeit der Nennung (2004/2005)

Quelle: Eigene Darstellung nach eigenen Berechnungen.

Das anhand der Ergebnisse der ersten Frage bereits zu erkennende Bild des sehr differenzierten individuellen Zeitaufwands der Landwirte zeigt sich folglich auch hier bei der Betrachtung des für das Verständnis der Ausschreibungsunterlagen und der spezifischen Anforderungen benötigten Zeitaufwands.

„Wie lange haben Sie ungefähr für die Ermittlung Ihres Angebots (Auswahl der ökologischen Güter und Kalkulation des Angebotspreises, jedoch noch ohne die Ausschreibungsunterlagen auszufüllen) benötigt?““

Die Ermittlung ihres Angebots stellt für die Landwirte die zentrale Aufgabe dar. Sie umfasst die Auswahl, welche ökologischen Güter auf welchen Flächen und zu welchem Preis pro Hektar angeboten werden. 
Die Frage nach dem für die Angebotsermittlung benötigten Zeitaufwand haben 28 Landwirte beantwortet und wie Tabelle 8-3 zeigt, hat sich hierbei eine sehr große Spanne ergeben, die den Bereich von 5 Minuten bis 400 Minuten umfasst und eine Standardabweichung von 104,48 Minuten aufweist. Als Mittelwert hat sich ein Zeitaufwand von rund 91 Minuten herauskristallisiert und der Median beträgt 55 Minuten.

Tab. 8-3: Zeitaufwand - Angebotsermittlung (2004/2005)

\begin{tabular}{|l|c|c|c|c|c|}
\hline $\mathrm{n}=\mathbf{2 8}$ & Min. & Max. & Mittelwert & Median & Std.abw. \\
\hline Minuten & 5 & 400 & 90,71 & 55 & 104,48 \\
\hline
\end{tabular}

Quelle: Eigene Berechnungen.

Die auch hier erkennbaren großen subjektiven Unterschiede des benötigten Zeitaufwands werden ebenfalls bei der in Abbildung 8-3 vorgenommenen grafischen Veranschaulichung sichtbar und verdeutlichen schon jetzt die Problematik der praktischen Ermittlung eines einheitlichen Werts für die Höhe des Zeitaufwands.

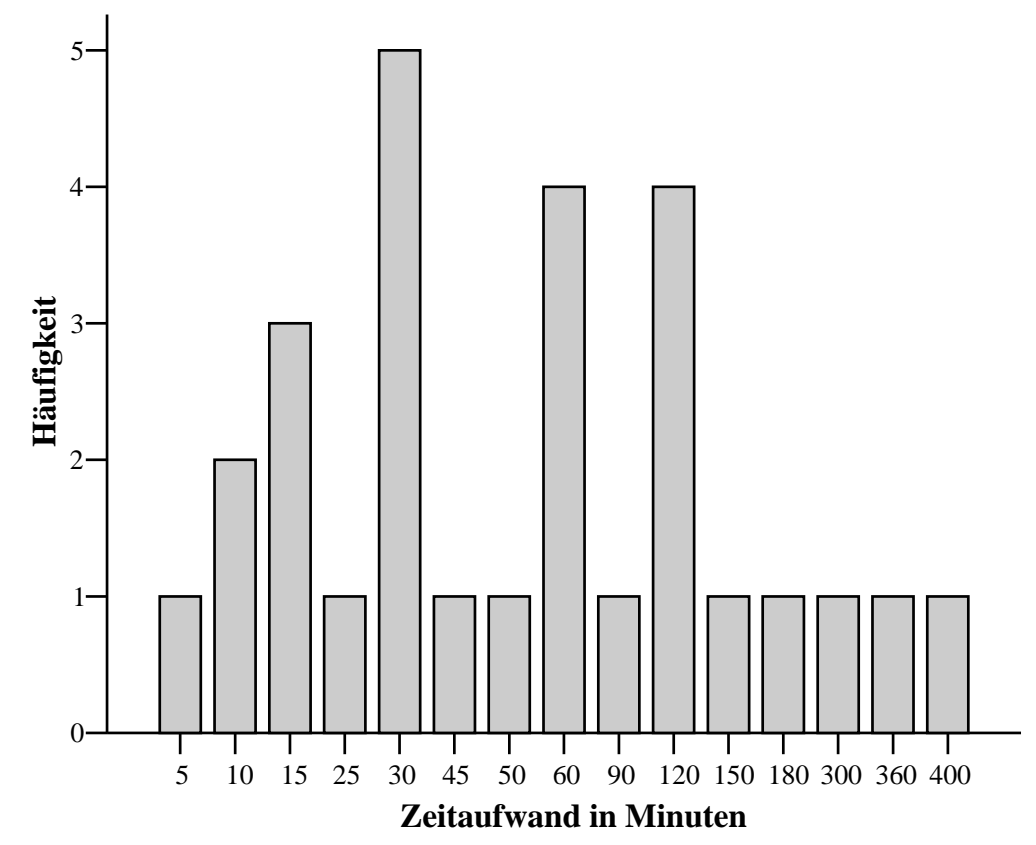

Abb. 8-3: Zeitaufwand zur Angebotsermittlung in Minuten und Häufigkeit der Nennung (2004/2005)

Quelle: Eigene Darstellung nach eigenen Berechnungen.

Hinsichtlich dieses zentralen Aufgabenbereichs innerhalb der Angebotsabgabe ist anzumerken, dass sich die große Differenz des Zeitaufwands teilweise in der unterschiedlichen Anzahl der angebotenen Schläge begründen lässt, da für jeden 
Schlag ein spezifisches Angebot ermittelt werden musste. Diese Angebotsermittlung umfasst neben der unmittelbaren Preiskalkulation vorab in der Regel auch die Begutachtung der anzubietenden Flächen bezüglich der Einstufung in die unterschiedlichen Qualitätsstufen ökologischer Güter, was je nach Anzahl der Flächen die erheblichen Unterschiede des Zeitaufwands erklärt. ${ }^{146}$

„Wie lange haben Sie ungefähr für das unmittelbare Ausfüllen der Ausschreibungsunterlagen (Antrag mit allen Anlagen) benötigt?“

Als vierte Abstufung im Prozess der Angebotsabgabe wird das letztliche Ausfüllen der gesamten Ausschreibungsunterlagen betrachtet. Die entsprechende Frage haben 28 Landwirte beantwortet und der Zeitaufwand umfasst mit einer Standardabweichung von 66,27 Minuten eine Spanne von 10 Minuten bis 300 Minuten. Der Mittelwert beträgt 77,50 Minuten und der Median 60 Minuten. Eine entsprechende Gegenüberstellung der Ergebnisse gibt Tabelle 8-4.

Tab. 8-4: Zeitaufwand - Ausfüllen der Ausschreibungsunterlagen (2004/2005)

\begin{tabular}{|l|c|c|c|c|c|}
\hline $\mathrm{n}=28$ & Min. & Max. & Mittelwert & Median & Std.abw. \\
\hline Minuten & 10 & 300 & 77,50 & 60 & 66,27 \\
\hline
\end{tabular}

Quelle: Eigene Berechnungen.

Wie bei der vorherigen Frage ist auch hier zu berücksichtigen, dass die ebenfalls in Abbildung 8-4 ersichtliche große Differenz des Zeitaufwands teilweise auf die unterschiedliche Anzahl der jeweils angebotenen Schläge zurückzuführen ist. Weiterhin umfasst das Ausfüllen der Ausschreibungsunterlagen, neben der Anforderung für jeden Schlag ein Angebot abzugeben, unter Umständen auch die Notwendigkeit Vervielfältigungen der Unterlagen zu erstellen sowie Kopien der aktuellen Luftbilder anzufertigen und dort die angebotenen Schläge kenntlich zu machen. $^{147}$

\footnotetext{
${ }^{146}$ Auf den Aspekt einer unterschiedlichen Anzahl von Angeboten der Landwirte wird in Abschnitt 8.6 bei der Ermittlung der Transaktionskosten ausführlich eingegangen.

${ }^{147}$ Dieser Aspekt wird hier ebenfalls nur kurz angerissen und in Abschnitt 8.6 erneut aufgegriffen.
} 


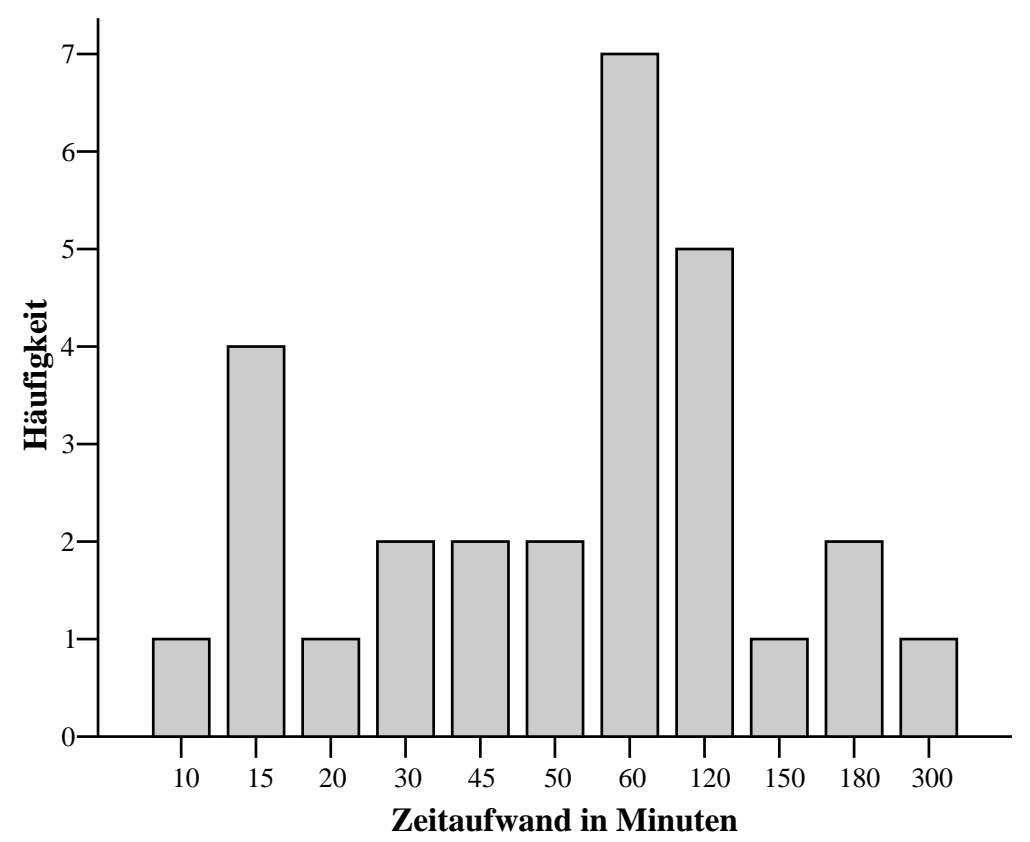

Abb. 8-4: Zeitaufwand zum Ausfüllen der Ausschreibungsunterlagen in Minuten und Häufigkeit der Nennung (2004/2005)

Quelle: Eigene Darstellung nach eigenen Berechnungen.

Mit der Betrachtung des Ausfüllens der Ausschreibungsunterlagen ist die Ermittlung des Zeitaufwands der Angebotserstellung abgeschlossen, denn nachfolgend mussten die ausgefüllten Unterlagen seitens der Landwirte lediglich in einem vorfrankierten Rückumschlag an die Vergabestelle, hier die Universität Göttingen, geschickt werden und eine nachträgliche Änderung des Angebots war nicht mehr möglich.

„Wie bewerten Sie persönlich den Zeitaufwand der Teilnahme an diesem Ausschreibungsverfahren im Vergleich zu der Antragstellung im Rahmen von Agrarumweltprogrammen?“

Über die Quantifizierung des Zeitaufwands hinaus wurde in der ersten Befragung durch eine gesonderte Frage ein Vergleich des Zeitaufwands mit bisherigen Antragstellungen in Agrarumweltprogrammen durchgeführt.

Für diesen Vergleich wurden die 17 Landwirte befragt, welche bislang sowohl mindestens einmal an Agrarumweltprogrammen als auch am Ausschreibungsverfahren 2004/2005 teilgenommen haben und ihnen als Antwort fünf mögliche Abstufungen vorgegeben. Alle 17 Landwirte haben eine Einschätzung abgegeben und die Ergebnisse sind in Tabelle 8-5 gegenübergestellt. 
Tab. 8-5: Vergleich des Zeitaufwands der Angebotsabgabe mit bisherigen Antragstellungen in Agrarumweltprogrammen (2004/2005)

\begin{tabular}{|c|c|c|c|c|}
\hline Viel höher & Höher & Gleich & Geringer & Viel geringer \\
\hline $3(17,6 \%)$ & $3(17,6 \%)$ & $5(29,4 \%)$ & $5(29,4 \%)$ & $1(5,9 \%)$ \\
\hline
\end{tabular}

Quelle: Eigene Berechnungen.

Es zeigt sich ein uneinheitliches Bild, denn jeweils drei Landwirte und damit ein Anteil von 17,6 \% haben den benötigten Zeitaufwand als viel höher oder höher bewertet. Eine ebenfalls identische Anzahl von fünf Landwirten und ein Anteil von je 29,4 \% hat die Angebotsabgabe als gleich oder weniger zeitaufwändig empfunden. Ein Landwirt erachtet den fraglichen Zeitaufwand als viel geringer.

Bei der Interpretation der Ergebnisse ist zu berücksichtigen, dass sich die Landwirte das erste Mal mit einem Ausschreibungsverfahren und in Folge dessen ebenfalls mit einigen grundlegend neuen Anforderungen konfrontiert gesehen haben. Somit kann an dieser Stelle darauf geschlossen werden, dass der Zeitaufwand im Rahmen der ersten Ausschreibung in der Tendenz ähnlich dem von Antragstellungen in bisherigen Agrarumweltprogrammen einzuschätzen ist. ${ }^{148}$

„Verfügen Sie über einen Internet-Zugang?“ / „Können Sie sich vorstellen, eine Antragstellung - beispielsweise im Rahmen von Agrarumweltprogrammen digital und per Internet vorzunehmen?“

Abschließend wird der Blick auf eine Möglichkeit gelenkt, die zukünftig eine transaktionskostenmindernde Abwicklung sowohl der Angebotsabgabe in einem Ausschreibungsverfahren als auch traditioneller Antragstellungen erwarten lässt; eine softwaregestützte Antragstellung und die Nutzung des Internets zur Datenübermittlung. Entscheidend sind dafür auf Seiten der Landwirte das Vorhandensein entsprechender technischer Voraussetzungen und eine grundsätzliche Bereitschaft zur Anpassung des Vorgehens. Vorteile sind in einem derartigen Verfahren darin zu sehen, dass sämtliche Formulare online abgerufen und zudem nicht veränderte Daten aus vorherigen Antragstellungen unmittelbar übernommen werden können, wodurch eine Reduzierung des Zeitaufwands der Antragstellung zu erwarten ist.

\footnotetext{
${ }^{148}$ Zudem sind im Rahmen weiterer Ausschreibungsrunden transaktionskostenmindernde Lerneffekte auf Seiten der Landwirte zu erwarten, die nach der Einbeziehungen der entsprechenden Befragungsergebnisse zum zweiten Ausschreibungsverfahren diskutiert werden.
} 
Insgesamt haben alle 35 Landwirte die Frage zum Vorhandensein eines InternetAnschlusses beantwortet und mit 25 Landwirten haben 71,4 \% angegeben, über einen solchen zu verfügen. Wie Tabelle 8-6 hinsichtlich der Bereitschaft zu einer digitalisierten Angebotsabgabe und der Nutzung des Internets zeigt, sind nicht alle der über einen Internet-Zugang verfügenden Landwirte auch bereit diesen entsprechend zu nutzen. Von den 35 Landwirten zeigen hier 22 und somit 62,9 \% eine derartige Bereitschaft.

Tab. 8-6: Verfügbarkeit eines Internet-Zugangs und Bereitschaft zur OnlineAntragstellung (2004/2005)

\begin{tabular}{|l|c|c|}
\hline $\mathrm{n}=35$ & Ja & Nein \\
\hline Internet-Zugang & $25(71,4 \%)$ & $10(28,6 \%)$ \\
\hline Online-Antragstellung & $22(62,9 \%)$ & $13(37,1 \%)$ \\
\hline Quelle: Eigene Berechnungen.
\end{tabular}

Demzufolge ist festzuhalten, dass diese Ergebnisse dafür sprechen, den Landwirten die optionale Möglichkeit einer softwaregestützten und internetbasierten Antragstellung zu schaffen, um in Folge dessen sehr wahrscheinlich den Zeitaufwand und die Transaktionskosten der Antragstellung reduzieren zu können.

\subsection{Die zweite Befragung}

Im Nachfolgenden werden eingangs die Konzeption der zweiten Befragung und einige Daten der teilnehmenden Landwirte dargestellt. Im Anschluss wird auf die für die Ermittlung und Bewertung der Transaktionskosten in der ersten Ausschreibungsrunde relevanten Ergebnisse eingegangen.

\subsubsection{Konzeption und teilnehmende Landwirte}

Die zweite schriftliche Befragung hat nach der Zuschlagserteilung im Herbst 2005 stattgefunden. Es wurden im Zuge dessen die 38 Landwirte angeschrieben, die innerhalb der Ausschreibungsrunde 2004/2005 ein fristgerechtes und formal korrektes Angebot abgegeben haben. ${ }^{149}$

\footnotetext{
${ }^{149}$ Der gesamte Fragebogen findet sich im Anhang 4 der Arbeit.
} 
Von den angeschriebenen 38 Landwirten haben 24 Landwirte an der Befragung teilgenommen, was einem Anteil von 63,2 \% entspricht. Hinsichtlich der Struktur der teilnehmenden Landwirte stellt sich folgende Unterteilung in Haupt- und Nebenerwerbslandwirte sowie in konventionell und ökologisch wirtschaftende Landwirte dar. Jeweils 12 der teilnehmenden Betriebe sind Haupt- und Nebenerwerbsbetriebe. 15 Betriebe verfolgen eine konventionelle und neun eine ökologische Landwirtschaft. In Agrarumweltprogramme haben bislang elf der 24 an der Befragung teilnehmenden Landwirte Flächen eingebracht.

\subsubsection{Ergebnisse}

Nachfolgend werden die im Hinblick auf die Transaktionskosten relevanten Ergebnisse der zweiten Befragung einbezogen und erneut das Vorgehen gewählt, jeweils die Frage aus dem Fragebogen als Zitat voranzustellen und im Anschluss die Ergebnisse zu diskutieren.

\section{„Welchen Stundenlohn schätzen Sie für sich persönlich als angemessen ein, wenn Sie für die Antragstellung unmittelbar bezahlt würden?“}

Für die Berechnung der Transaktionskosten ist es notwendig, den oben ermittelten Zeitaufwand der Landwirte für ihre Angebotsabgabe monetär zu bewerten. Hierzu wird der Ansatz verfolgt, die eigene Einschätzung der Landwirte einzubeziehen und sie wurden gebeten eine Angabe zu machen, welchen Stundenlohn sie für sich selbst als Entlohnung für die Angebotsabgabe als angemessen einschätzen.

21 Landwirte haben eine Angabe gemacht und der genannte Stundenlohn umfasst Werte von $10 €$ bis $25 €$, woraus sich ein Mittelwert von 16,76 € und ein Median von $15 €$ ergibt. Die Standardabweichung beträgt 5,39 €. Einen Überblick über die Ergebnisse gibt Tabelle 8-7.

Tab. 8-7: Als angemessen eingeschätzter Stundenlohn für die Angebotsabgabe $(2004 / 2005)$

\begin{tabular}{|l|c|c|c|c|c|}
\hline $\mathrm{n}=21$ & Min. & Max. & Mittelwert & Median & Std.abw. \\
\hline Euro & 10 & 25 & 16,76 & 15 & 5,39 \\
\hline
\end{tabular}

Quelle: Eigene Berechnungen. 
Ähnlich zu dem ermittelten Zeitaufwand der Angebotsabgabe zeigt sich hier für den individuellen Stundenlohn eine Differenz der von den Landwirten getätigten Einschätzungen, welche in Abbildung 8-5 dargestellt sind.

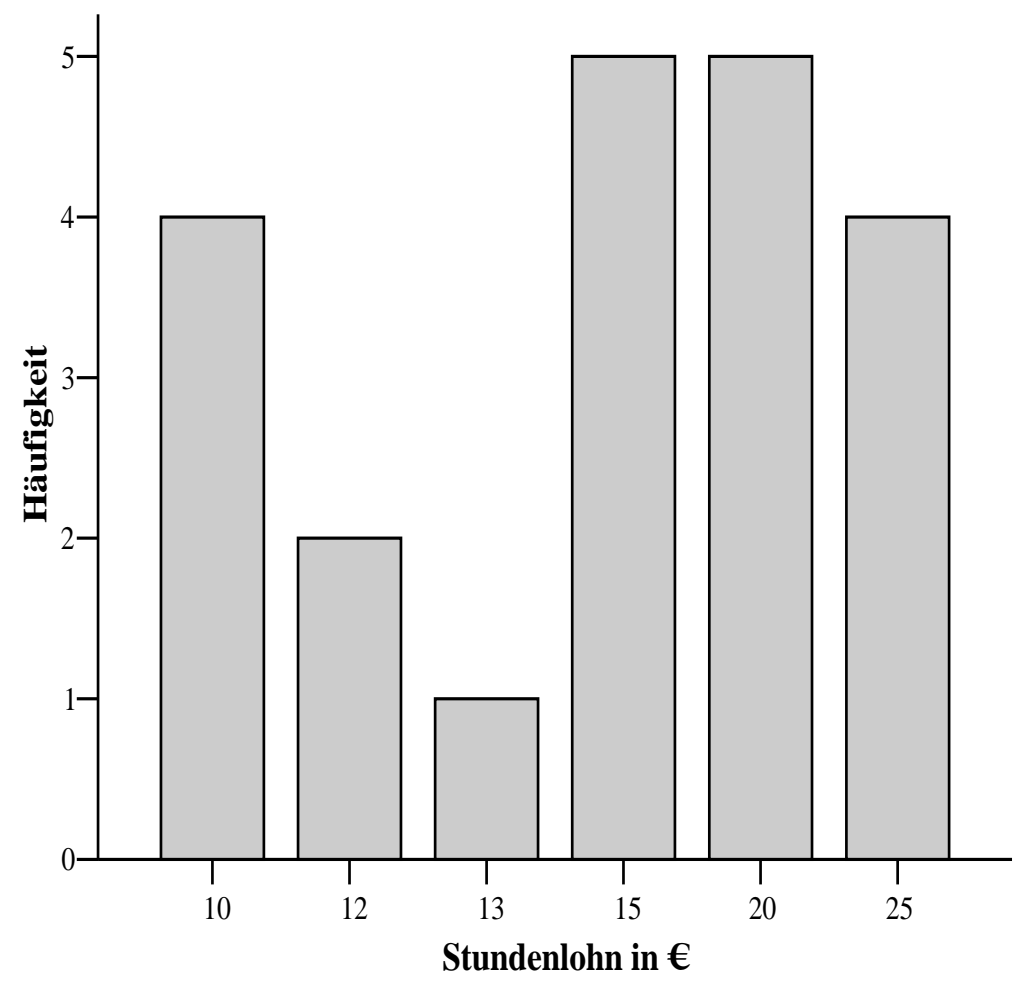

Abb. 8-5: Als angemessen eingeschätzter Stundenlohn für die Angebotserstellung in $€$ und Häufigkeit der Nennung (2004/2005)

Quelle: Eigene Darstellung nach eigenen Berechnungen.

Die Ergebnisse des von den Landwirten als angemessen eingeschätzten Stundenlohns werden in Abschnitt 8.6 zur Ermittlung der Transaktionskosten herangezogen und ferner hinsichtlich ihres spezifischen Einflusses auf die Höhe der Transaktionskosten diskutiert.

„Haben Sie bei der Kalkulation des Angebotspreises den von Ihnen zur Antragstellung benötigten Zeitaufwand bewertet und berücksichtigt?“

Die bereits im Zuge der Preiskalkulation der Landwirte angesprochene Frage, ob der für die Angebotsabgabe notwendige Zeitaufwand auch bei der Preiskalkulation berücksichtigt wurde, wird hier erneut aufgegriffen.

Insgesamt haben alle Landwirte die Frage beantwortet und elf von ihnen haben angegeben, den Zeitaufwand kalkuliert und berücksichtigt zu haben, was einem Anteil von 45,8 \% entspricht. Die Ergebnisse sind in Tabelle 8-8 dargestellt, wobei 
hinsichtlich einer Differenzierung in konventionell und ökologisch wirtschaftende Betriebe kein wesentlicher Unterschied ausgemacht werden kann.

Ein deutlich abweichendes Bild zeigt sich bei der Betrachtung von Haupt- und Nebenerwerbsbetrieben. Hier haben 75,0 \% der Haupterwerbslandwirte angegeben, den Zeitaufwand bzw. die Transaktionskosten der Angebotsabgabe bei der Preiskalkulation berücksichtigt zu haben. Im Gegensatz dazu haben lediglich 16,7 \% der im Nebenerwerb wirtschaftenden Landwirte diesen Zeitaufwand einkalkuliert.

Tab. 8-8: Berücksichtigung des Zeitaufwands der Angebotsabgabe bei der Preiskalkulation - Differenziert nach Bewirtschaftungs- und Betriebsform (2004/2005)

\begin{tabular}{|l|r|r|}
\hline $\mathrm{n}=24$ & Ja & Nein \\
\hline Gesamt & $11(45,8 \%)$ & $13(54,2 \%)$ \\
\hline Konventionell & $6(40,0 \%)$ & $9(60,0 \%)$ \\
\hline Ökologisch & $5(55,6 \%)$ & $4(44,4 \%)$ \\
\hline Haupterwerb & $9(75,0 \%)$ & $3(25,0 \%)$ \\
\hline Nebenerwerb & $2(16,7 \%)$ & $10(83,3 \%)$ \\
\hline
\end{tabular}

Quelle: Eigene Berechnungen.

Demzufolge zeigt sich, dass in der ersten Ausschreibungsrunde lediglich 45,8 \% der Landwirte die Transaktionskosten der Angebotsabgabe in ihren Angebotspreis einkalkuliert haben, was die generelle Berücksichtigung und Relevanz dieser Transaktionskosten diskutieren lässt.

Vor diesem Hintergrund ist erneut die differenzierte Betrachtung von Haupt- und Nebenerwerbslandwirten aufschlussreich, denn wie oben zu sehen ist, hat mit $75 \%$ der überwiegende Teil der Haupterwerbslandwirte den Zeitaufwand einkalkuliert, jedoch nur 16,7 \% der Nebenerwerbslandwirte. Dies spricht dafür, dass die Haupterwerbslandwirte bereits in dieser ersten Ausschreibungsrunde weitestgehend unternehmerisch kalkuliert haben und für die Nebenerwerbslandwirte eine Deckung der spezifischen Transaktionskosten weniger relevant ist, da sie ihr Einkommen maßgeblich anderweitig realisieren und somit innerhalb eines Ausschreibungsverfahrens relativ geringere Preise anbieten können und demzufolge ihre Chancen erhöhen, einen Zuschlag zu erhalten. ${ }^{150}$

\footnotetext{
150 Da die identische Frage innerhalb der dritten Befragung erneut gestellt wurde, erfolgt eine abschließende Betrachtung unter Einbeziehung dieser Ergebnisse im Rahmen der zusammenfassenden Diskussion in Abschnitt 8.7.
} 
„Wie schätzen Sie für sich persönlich den vermutlich notwendigen Zeitaufwand der Teilnahme an einer kommenden Ausschreibungsrunde - verglichen mit der letzten Ausschreibungsrunde - ein?“

Alle 24 Landwirte haben die Frage beantwortet und mit 17 Landwirten hat die große Mehrheit von 70,8 \% angegeben, dass sie den Zeitaufwand im Rahmen einer zukünftigen im Vergleich mit der abgeschlossenen Ausschreibungsrunde als gleich ansehen. Vier Landwirte und somit 16,7 \% erwarten, dass sich der Zeitaufwand verringern wird und mit drei Landwirten erwarten $12,5 \%$ einen größeren Zeitaufwand bei einer kommenden Ausschreibungsrunde. Tabelle 8-9 stellt die Einschätzungen zur Entwicklung des Zeitaufwands der Angebotserstellung gegenüber.

Tab. 8-9: Vergleich des vermutlichen Zeitaufwands der Teilnahme an der kommenden Ausschreibung im Vergleich zu der Ausschreibung 2004/2005

\begin{tabular}{|c|c|c|}
\hline Geringer & Gleich & Höher \\
\hline $4(16,7 \%)$ & $17(70,8 \%)$ & $3(12,5 \%)$ \\
\hline
\end{tabular}

Quelle: Eigene Berechnungen.

Für die drei einen größeren Zeitaufwand erwartenden Landwirte lässt sich die Erklärung finden, dass sie innerhalb des ersten Ausschreibungsverfahrens noch nicht alle möglichen Schläge angeboten haben. Eine zukünftige Einbeziehung dieser Flächen würde bedingt durch die schlagbezogene Angebotsabgabe zu einem höheren Zeitaufwand durch zusätzliche Angebotsermittlungen und weitere auszufüllende Formulare führen. Eine von 17 Landwirten abgegebene Erwartung eines gleich bleibenden Zeitaufwands lässt darauf schließen, dass diese Landwirte bereits in der ersten Ausschreibungsrunde ihre relevanten Flächen in der Modellregion angeboten haben und die Bearbeitung der Ausschreibungsunterlagen ihnen relativ leicht gefallen ist, so dass hier keine Lerneffekte zu erwarten sind. Demgegenüber deutet die von vier Landwirten erwartete Reduzierung des Zeitaufwands auf zukünftige transaktionskostenmindernde Lerneffekte hin. 


\subsection{Die dritte Befragung}

Die zur Ermittlung und Bewertung der Transaktionskosten der zweiten Ausschreibungsrunde herangezogenen Daten beruhen auf einer weiteren schriftlichen Befragung, die im Zeitraum Mitte Februar bis Ende März 2006 parallel zur Angebotsabgabe durchgeführt wurde. Nachfolgend wird sowohl auf die Konzeption der Befragung als auch auf die teilnehmenden Landwirte eingegangen und im Anschluss werden die wesentlichen Befragungsergebnisse betrachtet. ${ }^{151}$

\subsubsection{Konzeption und teilnehmende Landwirte}

Mit dem Ziel, die im Verlauf der Ausschreibungsrunde 2004/2005 ermittelten Transaktionskosten zu verifizieren bzw. ihre Veränderung aufzuzeigen und diskutieren zu können, wurde während des Ausschreibungsverfahrens 2006 eine dritte schriftliche Befragung von Landwirten durchgeführt. ${ }^{152}$

Hierzu wurden alle innerhalb der bereits in Kapitel Sieben thematisierten beschränkten Ausschreibung 2006 einbezogenen Landwirte angeschrieben und ihnen zusammen mit den Ausschreibungsunterlagen ein anonymer Fragebogen zugeschickt. Zur Ermittlung des für die Angebotsabgabe notwendigen Zeitaufwands wurden die Landwirte erneut gebeten, parallel zum Ausfüllen der Ausschreibungsunterlagen auch den Zeitaufwand innerhalb des Fragebogens zu notieren und weitergehende Angaben zu machen.

Von den angeschriebenen 39 Landwirten haben 27 den Fragebogen ausgefüllt zurückgesandt, was einem Anteil von 69,2 \% entspricht. Davon wiederum haben 25 Landwirte im Rahmen des Ausschreibungsverfahrens ein Angebot eingereicht und sie stellen die für die folgenden Ausführungen relevanten Landwirte dar. ${ }^{153}$ Insgesamt haben 30 Landwirte ein Angebot abgegeben und demzufolge sind 83,3 \% dieser Landwirte innerhalb der Befragung repräsentiert. Für die relevanten landwirtschaftlichen Betriebe zeigt sich bezüglich der Betriebsform die Unterteilung, dass neun Landwirte ihren Betrieb im Haupt- und 16 Landwirte im Nebenerwerb

\footnotetext{
${ }^{151}$ Der gesamte Fragebogen findet sich im Anhang 5 der Arbeit.

${ }^{152}$ Im Zuge dessen wurden sowohl die zur Preiskalkulation und dem Bieterverhalten der Landwirte als auch die zur Ermittlung der Transaktionskosten relevanten Fragen aus den ersten beiden oben angesprochenen Befragungen erneut und zusammengefasst in einem Fragebogen gestellt.

153 Sofern keine abweichenden Angaben gemacht werden, beziehen sich alle nachfolgenden Ausführungen des Kapitels auf diese Landwirte.
} 
führen. 16 Landwirte betreiben eine konventionelle und neun Landwirte eine ökologische Landwirtschaft.

\subsubsection{Ergebnisse}

Für die Diskussion der Befragungsergebnisse wird erneut ein Vorgehen gewählt, bei dem die Fragen in der im Fragebogen verwendeten Form jeweils als Zitat vorangestellt und anschließend die Ergebnisse betrachtet werden.

„Wie viel Zeit haben Sie ungefähr für die Beschaffung der Ausschreibungsunterlagen benötigt?“

Bedingt durch die Wahl einer auf den Kreis der Bieter beschränkten Ausschreibung, die bereits im Rahmen des ersten Ausschreibungsverfahrens ein Angebot abgegeben haben, wurden die Ausschreibungsunterlagen den Landwirten direkt zugeschickt. Aus diesem Grund entfallen an dieser Stelle die im Rahmen des ersten Ausschreibungsverfahrens noch berücksichtigten Angaben zur Beschaffung der Ausschreibungsunterlagen.

\section{„Wie lange haben Sie ungefähr für das Lesen und Verstehen der gesamten} Ausschreibungsunterlagen (ohne Kalkulation des Angebotspreises und Ausfüllen der Ausschreibungsunterlagen) benötigt?“

Infolge der beschränkten Ausschreibung und des damit verbundenen Vorgehens stellt das Lesen und grundsätzliche Verstehen der Ausschreibungsunterlagen im Verlauf der Angebotserstellung die erste Anforderung an die Landwirte dar.

Gegenüber der ersten Ausschreibungsrunde erfolgte eine grundlegende Veränderung dahingehend, dass das System der Feldblockidentifikatoren auch hier zur Identifizierung der angebotenen Schläge entsprechend der aktuellen Betriebskarte der Landwirtschaftskammer eingeführt wurde. Darüber hinaus wurden keine wesentlichen Änderungen des Vorgehens und der Ausschreibungsunterlagen vorgenommen. Nichtsdestoweniger besteht für die Landwirte die Notwendigkeit, sich über die aktuellen Anforderungen im Zuge der Angebotsabgabe zu informieren und sich mit den Ausschreibungsunterlagen vertraut zu machen.

Die Frage nach dem dafür benötigten Zeitaufwand wurde von allen 25 Landwirten beantwortet und wie Tabelle 8-10 zeigt, stellt sich bereits hier ein sehr differenziertes Bild dar. Die Nennungen umfassen bei einer Standardabweichung von 24,32 
Minuten eine Spanne von 5 Minuten bis 120 Minuten, wobei der Mittelwert 33 Minuten und der Median 30 Minuten beträgt.

Tab. 8-10: Zeitaufwand - Lesen und Verstehen der Ausschreibungsunterlagen (2006)

\begin{tabular}{|l|c|c|c|c|c|}
\hline $\mathrm{n}=25$ & Min. & Max. & Mittelwert & Median & Std.abw. \\
\hline Minuten & 5 & 120 & 33,00 & 30 & 24,32 \\
\hline
\end{tabular}

Quelle: Eigene Berechnungen.

Eine grafische Veranschaulichung der Ergebnisse gibt Abbildung 8-6 durch die Gegenüberstellung des benötigten Zeitaufwands in Minuten und der Häufigkeit seiner jeweiligen Nennung.

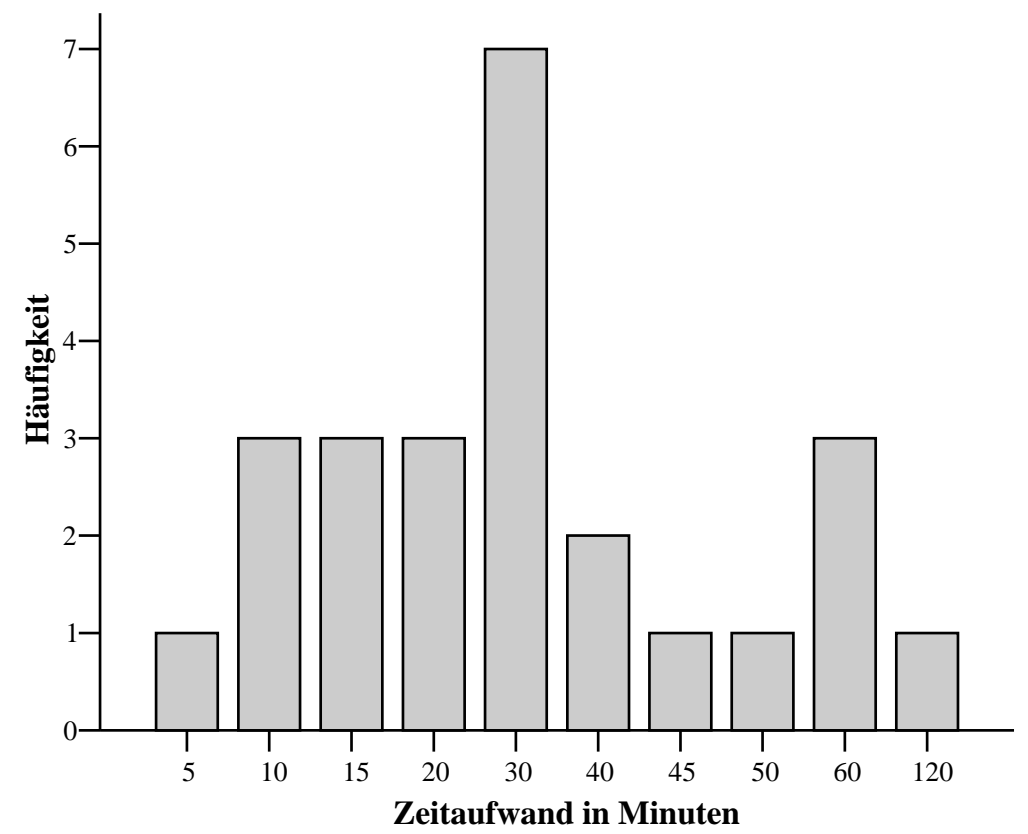

Abb. 8-6: Zeitaufwand zum Lesen und Verstehen der Ausschreibungsunterlagen in Minuten und Häufigkeit der Nennung (2006)

Quelle: Eigene Darstellung nach eigenen Berechnungen.

Die Ergebnisse zeigen die auch im Zuge der zweiten Ausschreibung unterschiedlich ausgeprägte Notwendigkeit des Auseinandersetzens mit den Ausschreibungsunterlagen und den damit verbundenen Anforderungen.

Diesbezüglich ist zudem zu berücksichtigen, dass im Rahmen der ersten Ausschreibungsrunde einige wenige Angebote aufgrund von Formfehlern (unvollständiges oder fehlerhaftes Ausfüllen der Ausschreibungsunterlagen) abgelehnt werden mussten und hier eine erneute intensive Auseinandersetzung mit 
den Ausschreibungsanforderungen zu erwarten ist. Dies hat dazu geführt, dass in der Ausschreibungsrunde 2006 keine Angebote aus formalen Gründen abgelehnt werden mussten.

„Wie lange haben Sie ungefähr für die Ermittlung Ihres Angebots (Auswahl der ökologischen Güter und Kalkulation des Angebotspreises, jedoch noch ohne die Ausschreibungsunterlagen auszufüllen) benötigt?“

Hinsichtlich dieser zentralen Anforderung haben alle 25 Landwirte eine Antwort gegeben und wie Tabelle 8-11 zu entnehmen ist, zeigt sich mit einer Standardabweichung von 71,53 Minuten weiterhin eine große Spanne des genannten und von 5 Minuten bis 300 Minuten reichenden Zeitaufwands.

Tab. 8-11: Zeitaufwand - Angebotsermittlung (2006)

\begin{tabular}{|l|c|c|c|c|c|}
\hline $\mathrm{n}=25$ & Min. & Max. & Mittelwert & Median & Std.abw. \\
\hline Minuten & 5 & 300 & 67,80 & 50 & 71,53 \\
\hline
\end{tabular}

Quelle: Eigene Berechnungen.

Für die weiteren Betrachtungen wird auf zwei entscheidende Werte fokussiert und demzufolge der Mittelwert in Höhe von 67,80 Minuten und der Median in Höhe von 50 Minuten einbezogen. Die grafische Veranschaulichung der Ergebnisse erfolgt in Abbildung 8-7 durch die Darstellung des benötigten Zeitaufwands und der jeweiligen Häufigkeit der Nennung. 


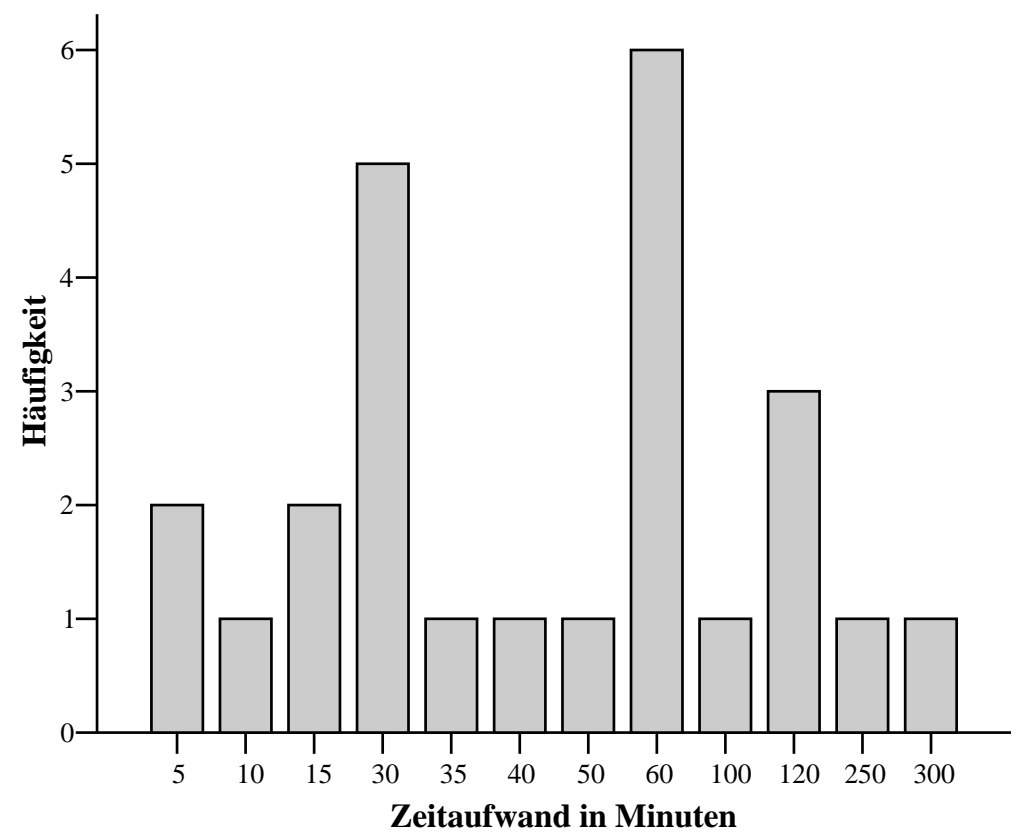

\section{Abb. 8-7: Zeitaufwand zur Angebotsermittlung in Minuten und Häufigkeit der Nennung (2006)}

Quelle: Eigene Darstellung nach eigenen Berechnungen.

Bei der Interpretation der Ergebnisse ist erneut $\mathrm{zu}$ beachten, dass durch die schlagbezogene Angebotsabgabe und Zuschlagserteilung für jeden Schlag ein individuelles Angebot ermittelt werden musste und eine entsprechend große Differenz des hierfür benötigten Zeitaufwands teilweise darin zu begründen ist. ${ }^{154}$ Darüber hinaus ist neu einzubeziehen, dass die Differenzen des jeweiligen Zeitaufwands in der zweiten Ausschreibungsrunde ebenfalls durch die Ergebnisse des ersten Ausschreibungsverfahrens und daraus folgende Anpassungen beeinflusst werden können.

Für die Landwirte, welche innerhalb der Ausschreibung 2004/2005 ein erfolgreiches Angebot abgegeben haben, ist zu erwarten, dass keine grundlegenden Anpassungen vorgenommen wurden und die Angebotsermittlung entsprechend rasch erfolgen konnte.

Demgegenüber stellt sich für die Landwirte, die entweder bereits bei der Zuschlagserteilung oder letztlich im Zuge der Kontrolle der ökologischen Leistung nicht ausschließlich erfolgreiche Angebote abgegeben haben, die Anforderung, diese Ergebnisse im Rahmen einer neuen Angebotsermittlung einzubeziehen. In der Folge

\footnotetext{
${ }^{154}$ Ein Aspekt, der in Kapitel 8.6 im Rahmen der Ermittlung und Bewertung der Transaktionskosten unter Einbeziehung der Anzahl der angebotenen Schläge erneut aufgegriffen wird.
} 
kann es sowohl hinsichtlich der ökologischen als auch der ökonomischen Angebotskomponente $\mathrm{zu}$ einer grundlegend neuen und zeitaufwändigen Angebotsermittlung kommen. Im Hinblick auf den dabei zu führenden Abwägungsprozess ist die Überlegung zu unterstellen, durch die Inkaufnahme einer geringeren Honorierung die Wahrscheinlichkeit der Zuschlagserteilung zu erhöhen oder für den Fall, dass dies zu keiner wirtschaftlich vertretbaren Produktion führen sollte, die fragliche Fläche nicht mehr anzubieten und sie einer anderweitigen Nutzung zuzuführen.

\section{„Wie lange haben Sie ungefähr für das unmittelbare Ausfüllen der Ausschreibungsunterlagen (Antrag mit allen Anlagen) benötigt?“}

Nachdem die wesentliche Aufgabe der Angebotsermittlung abgeschlossen ist, gilt es die Ausschreibungsunterlagen schlagbezogen auszufüllen und zur Identifizierung der Flächen ergänzende Kennzeichnungen innerhalb von Kopien der relevanten Luftbilder vorzunehmen.

Diese Frage wurde ebenfalls von allen 25 Landwirten beantwortet. Wie Tabelle 8-12 zeigt, ist es hierbei erneut $\mathrm{zu}$ einem uneinheitlichen Bild gekommen und der genannte Zeitaufwand umfasst bei einer Standardabweichung von 51,77 Minuten einen Bereich von 2 Minuten bis 180 Minuten.

Tab. 8-12: Zeitaufwand - Ausfüllen der Ausschreibungsunterlagen (2006)

\begin{tabular}{|l|c|c|c|c|c|}
\hline $\mathrm{n}=25$ & Min. & Max. & Mittelwert & Median & Std.abw. \\
\hline Minuten & 2 & 180 & 64,28 & 60 & 51,77 \\
\hline
\end{tabular}

Quelle: Eigene Berechnungen.

Für die nachfolgenden Berechnungen werden der sich aus den in Abbildung 8-8 dargestellten Angaben zum Zeitaufwand des Ausfüllens der Ausschreibungsunterlagen ergebende Mittelwert von 64,28 Minuten und der Median von 60 Minuten herangezogen. 


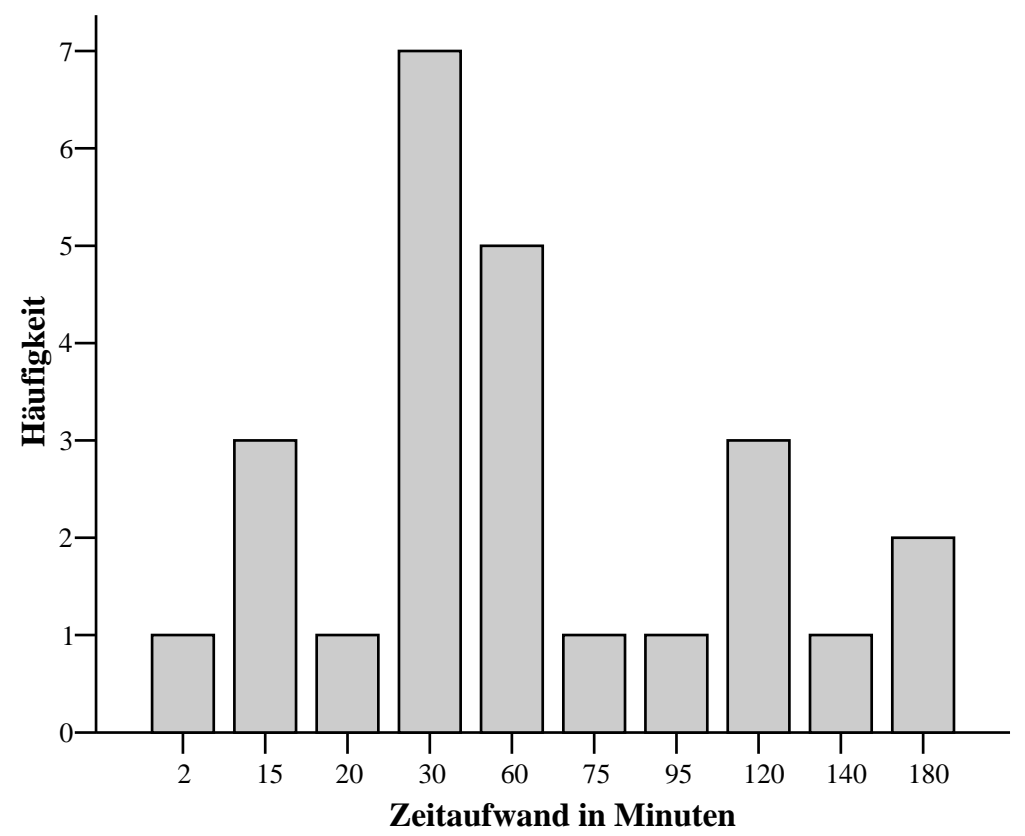

Abb. 8-8: Zeitaufwand zum Ausfüllen der Ausschreibungsunterlagen in Minuten und Häufigkeit der Nennung (2006)

Quelle: Eigene Darstellung nach eigenen Berechnungen.

Zur Erklärung der Ergebnisse ist neben den grundsätzlichen individuellen Differenzen der Landwirte erneut die Anzahl der jeweils angebotenen Schläge zu nennen und mit einer steigenden Anzahl ebenfalls ein Ansteigen des gesamten Zeitaufwands zu erwarten. ${ }^{155}$

Zudem ist für einen Landwirt anzumerken, dass er bereits im Rahmen der ersten Ausschreibung darum gebeten hatte, ihm die auszufüllenden Ausschreibungsunterlagen als Datei per E-Mail zukommen zu lassen, um die entsprechenden Daten zu seinen Flächen auch für zukünftige Ausschreibungen übernehmen zu können. Ein Aspekt, der hier exemplarisch das Potential für transaktionskostenmindernde Effekte eines derartigen Vorgehens verdeutlicht und langfristig weiter verfolgt werden sollte.

155 Dieser Aspekt wird nachfolgend im Rahmen der Transaktionskostenermittlung erneut explizit aufgegriffen. Der auffallend geringe Zeitaufwand von nur zwei Minuten ist in der Abgabe eines lediglich einen Schlag umfassenden und im Vergleich zum Vorjahr unveränderten Angebots zu erklären. 
„Welchen Stundenlohn schätzen Sie für sich persönlich als angemessen ein, wenn Sie für die Antragstellung unmittelbar bezahlt würden?“

Eine Einschätzung des als angemessen erachteten Stundenlohns haben 24 der 25 Landwirte abgegeben und die genannten Stundenlöhne umfassen mit einer Standardabweichung von 12,41 € eine Spanne von $10 €$ bis $60 €$. Einen Überblick der für die monetäre Bewertung wesentlichen Ergebnisse gibt Tabelle 8-13.

Tab. 8-13: Als angemessen eingeschätzter Stundenlohn für die Angebotsabgabe (2006)

\begin{tabular}{|l|c|c|c|c|c|}
\hline $\mathrm{n}=24$ & Min. & Max. & Mittelwert & Median & Std.abw. \\
\hline Euro & 10 & 60 & 21,42 & 20 & 12,41 \\
\hline
\end{tabular}

Quelle: Eigene Berechnungen.

Wie Abbildung 8-9 verdeutlicht, ist es seitens der Landwirte zu einer heterogenen Einschätzung des Stundenlohns gekommen und mit jeweils einer Nennung von $50 €$ bzw. $60 €$ sind zwei sehr hohe Werte in die Auswertung einzubeziehen.

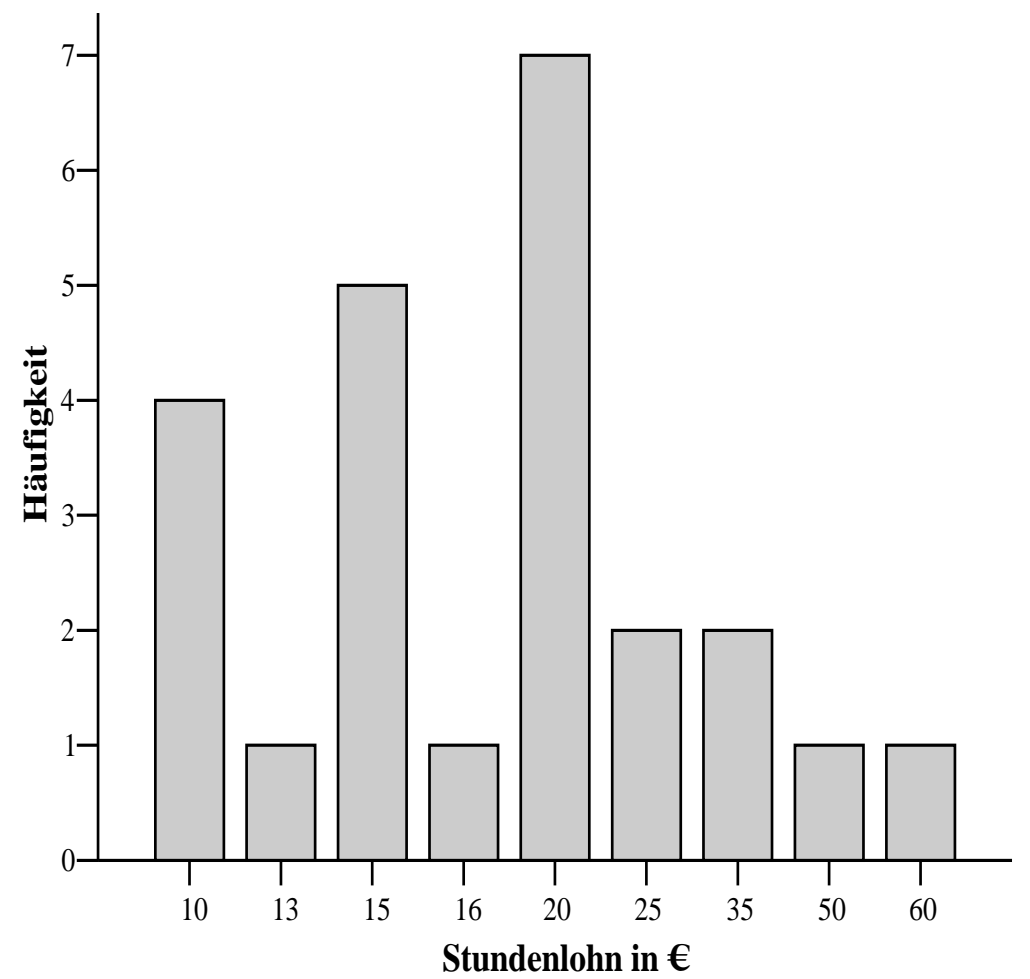

Abb. 8-9: Als angemessen eingeschätzter Stundenlohn für die Angebotserstellung in $€$ und Häufigkeit der Nennung (2006)

Quelle: Eigene Darstellung nach eigenen Berechnungen. 
Zur Einordnung der Ergebnisse ist neu berücksichtigen, dass innerhalb der zweiten Ausschreibung erstmals zwei wesentlich über die in der Regel als angemessen einzuschätzende Spanne eines Stundenlohns hinausgehende Werte genannt wurden. Demzufolge wird weiterhin die grundsätzlich vorhandene unterschiedliche Bewertung des Stundenlohns und damit verbunden eine divergierende individuelle Wertschätzung seitens der Landwirte deutlich. Gleichzeitig wirft dies die Frage auf, ob von den Landwirten bei ihrer Einschätzung lediglich die ursprünglich fokussierte Honorierung ihrer unmittelbaren Tätigkeit im Zuge der Angebotserstellung oder weitere Aspekte wie Fahrtkosten im Rahmen der Begutachtung der Flächen einbezogen und durch den genannten Stundenlohn honoriert werden sollen.

Für die beiden abweichend hohen Werte ist letzteres zu erwarten und es verdeutlicht die bei einem entsprechenden Vorgehen zur Datenermittlung zwangsläufig vorhandenen und im Zuge der nachfolgenden Diskussion der Transaktionskosten zu berücksichtigenden Ungenauigkeiten.

\section{„Haben Sie bei der Kalkulation des Angebotspreises den von Ihnen zur} Antragstellung benötigten Zeitaufwand bewertet und berücksichtigt?“

Alle 25 Landwirte haben diese Frage beantwortet und insgesamt zeigt sich, dass zwölf Landwirte den Zeitaufwand einkalkuliert haben, was einem Anteil von 48,0 \% entspricht.

Darüber hinaus wird zur Bewertung der Ergebnisse erneut hinsichtlich der Bewirtschaftungs- und Betriebsform unterschieden und aus den in Tabelle 8-14 dargestellten Antworten geht hervor, dass mit 62,5 \% und 10 Landwirten die überwiegende Zahl der konventionell wirtschaftenden Landwirte angegeben hat, den Zeitaufwand berücksichtigt zu haben. Von den ökologischen Betrieben haben dies lediglich zwei Landwirte angegeben, was einem Anteil von 22,2 \% entspricht. Differenziert nach Haupt- und Nebenerwerb zeigt sich für die Ausschreibung 2006 eine weniger eindeutige Unterscheidung, da vier und somit 44,4\% der im Haupterwerb und mit 50,0 \% acht der im Nebenerwerb wirtschaftenden Landwirte den Zeitaufwand der Angebotserstellung bewertet und innerhalb der Angebotskalkulation berücksichtigt haben. 
Tab. 8-14: Berücksichtigung des Zeitaufwands der Angebotsabgabe bei der Preiskalkulation - Differenziert nach Bewirtschaftungs- und Betriebsform (2006)

\begin{tabular}{|l|c|c|}
\hline $\mathrm{n}=25$ & Ja & Nein \\
\hline Gesamt & $12(48,0 \%)$ & $13(52,0 \%)$ \\
\hline Konventionell & $10(62,5 \%)$ & $6(37,5 \%)$ \\
\hline Ökologisch & $2(22,2 \%)$ & $7(77,8 \%)$ \\
\hline Haupterwerb & $4(44,4 \%)$ & $5(55,6 \%)$ \\
\hline Nebenerwerb & $8(50,0 \%)$ & $8(50,0 \%)$ \\
\hline
\end{tabular}

Quelle: Eigene Berechnungen.

Vor dem Hintergrund der bereits innerhalb der Ausschreibung 2004/2005 angesprochenen Frage der grundsätzlichen Relevanz der einzelbetrieblichen Transaktionskosten zeigt sich mit der Einbeziehung der obigen Ergebnisse eine Bestätigung der Tendenz, dass weniger als die Hälfte der teilnehmenden Landwirte diesen Zeitaufwand kalkuliert und in ihre Angebotspreise einbezogen hat. ${ }^{156}$

„Wie bewerten Sie persönlich den Zeitaufwand der Teilnahme an diesem Ausschreibungsverfahren im Vergleich zu der Antragstellung im Rahmen bisheriger Agrarumweltprogramme?“

Mit dem Ziel eines praktischen Institutionenvergleichs hinsichtlich des Teilbereichs der Angebotsabgabe innerhalb einer Ausschreibung bzw. der Antragstellung in bestehenden Agrarumweltprogrammen mit einer Einheitsprämie wurden die bislang sowohl an Agrarumweltprogrammen als auch der Ausschreibung 2006 teilnehmenden Landwirte um einen entsprechenden Vergleich des notwendigen Zeitaufwands gebeten. Alle 20 relevanten Landwirte haben die Frage beantwortet und die Ergebnisse sind in Tabelle 8-15 dargestellt.

Tab. 8-15: Vergleich des Zeitaufwands der Angebotsabgabe mit bisherigen Antragstellungen in Agrarumweltprogrammen (2006)

\begin{tabular}{|c|c|c|c|c|}
\hline Viel höher & Höher & Gleich & Geringer & Viel geringer \\
\hline $1(3,7 \%)$ & $7(25,9 \%)$ & $6(22,2 \%)$ & $5(18,5 \%)$ & $1(3,7 \%)$ \\
\hline
\end{tabular}

Quelle: Eigene Berechnungen.

\footnotetext{
${ }^{156}$ Eine abschließende Diskussion dieser Fragen wird unter Einbeziehung aller relevanten Ergebnisse innerhalb der zusammenfassenden Betrachtung in Abschnitt 8.7 erfolgen.
} 
Es zeigt sich weiterhin ein uneinheitliches Bild. Jeweils ein Landwirt und damit ein Anteil von 3,7 \% erachtet die Angebotsabgabe für viel höher bzw. viel geringer zeitaufwändig als eine Antragstellung. Ein Anteil von 25,9 \% und somit sieben Landwirte beurteilen den Zeitaufwand innerhalb einer Ausschreibung als höher und sechs Landwirte als gleich, was einem Anteil von 22,2 \% entspricht. Einen geringeren Zeitbedarf attestieren einer Ausschreibung fünf und demzufolge 18,5 \% der Landwirte.

Zusammenfassend zeigt sich eine sehr individuelle Einschätzung, wobei als Tendenz zu erkennen ist, dass trotz der in der Regel umfassenderen Anforderungen an die Landwirte im Fall einer Ausschreibung ein ähnlicher Zeitaufwand wie bei einer Einheitsprämie zu erwarten ist.

„Verfügen Sie über einen Internet-Zugang?“ / „Können Sie sich vorstellen, eine Antragstellung - beispielsweise im Rahmen von Agrarumweltprogrammen digital und per Internet vorzunehmen?“

Als abschließende Befragungsergebnisse werden mit der Perspektive einer zukünftigen internetbasierten und softwaregestützten Angebotsabgabe bzw. Antragstellung die technischen Voraussetzung und die notwendige Akzeptanz seitens der Landwirte betrachtet.

Die beiden Fragen wurden von allen 27 an der Befragung teilnehmenden Landwirten beantwortet und wie in Tabelle 8-16 dargestellt ist, haben insgesamt 21 Landwirte und somit 77,8 \% angegeben, über einen Internet-Zugang zu verfügen. Hinsichtlich der Bereitschaft von einer softwaregestützten Antragstellung unter Nutzung des Internets Gebrauch zu machen haben 19 Landwirte eine positive Einschätzung abgegeben, was einem Anteil von 70,4 \% entspricht.

Tab. 8-16: Verfügbarkeit eines Internet-Zugangs und Bereitschaft zur OnlineAntragstellung (2006)

\begin{tabular}{|l|c|c|}
\hline $\mathrm{n}=27$ & Ja & Nein \\
\hline Internet-Zugang & $21(77,8 \%)$ & $6(22,2 \%)$ \\
\hline Online-Antragstellung & $19(70,4 \%)$ & $8(29,6 \%)$ \\
\hline
\end{tabular}

Quelle: Eigene Berechnungen.

Die Ergebnisse zeigen auch hier, dass noch nicht alle landwirtschaftlichen Betriebe über einen Internet-Zugang verfügen und bereit sind, eine entsprechende 
Antragstellung zu nutzen. Nichtsdestoweniger lässt die positive Einschätzung des überwiegenden Anteils der Landwirte die Notwendigkeit erkennen, mittelfristig eine entsprechende Anpassung der Antragstellung vorzunehmen und bereits kurzfristig eine optionale Möglichkeit zu schaffen, um zu erwartende transaktionskostenmindernde Potentiale sowohl auf Seiten der Landwirte als letztlich auch seitens der Agrarverwaltung ausschöpfen zu können.

\subsection{Ermittlung der Transaktionskosten}

Nachdem für die beiden durchgeführten Ausschreibungen die notwendigen Befragungsergebnisse präsentiert wurden, erfolgt die Berechnung der mit der Angebotsabgabe verbundenen Transaktionskosten der Landwirte.

Bei der Diskussion der Befragungsergebnisse hat sich gezeigt, dass sowohl der individuelle Zeitaufwand als auch der genannte Stundenlohn mitunter erhebliche Abweichungen aufweisen. Um diesen Unterschieden Rechnung zu tragen und die Konsequenzen der Berechnungsgrundlage für die Höhe der Transaktionskosten diskutieren zu können, werden sowohl der Mittelwert als auch der Median des gesamten Zeitaufwands und des Stundenlohns berücksichtigt. Demzufolge werden für beide Ausschreibungsrunden jeweils zwei unterschiedliche Ermittlungen der Transaktionskosten vorgenommen, wobei das grundsätzliche Vorgehen unverändert bleibt und lediglich differierende Werte in Form der Mittelwerte und der Mediane die Grundlage bilden.

Im Zuge dessen werden eingangs jeweils die Transaktionskosten eines durchschnittlichen Betriebs berechnet, indem der gesamte Zeitaufwand mit dem als angemessen eingeschätzten Stundenlohn bewertet wird.

Um der unterschiedlichen Anzahl der von einem Betrieb angebotenen Schläge Rechnung zu tragen, werden in einem zweiten Schritt die Transaktionskosten je Schlag berechnet. Hierzu werden die Transaktionskosten des durchschnittlichen Betriebs mit der Anzahl der innerhalb der Befragung relevanten Landwirte multipliziert und die Summe anschließend durch die Anzahl der von diesen Landwirten angebotenen Schläge dividiert. 
Zum Abschluss werden die Transaktionskosten pro Schlag mit der Anzahl aller in der Ausschreibungsrunde angebotenen Schläge multipliziert und die entsprechenden gesamten Transaktionskosten der Angebotsabgabe ermittelt.

Zu ermittelnde Transaktionskosten:

1. Transaktionskosten je Betrieb

2. Transaktionskosten je Schlag

3. Gesamte Transaktionskosten

Im Zuge der Transaktionskostenermittlung wird demzufolge unterstellt, dass bedingt durch die schlagbezogene Angebotsabgabe für einen Landwirt nicht die Anzahl der angebotenen Hektar, sondern die Anzahl der angebotenen Schläge hinsichtlich des benötigten Zeitaufwands relevant ist.

\subsubsection{Transaktionskosten der Ausschreibung 2004/2005}

Der Transaktionskostenberechnung für die erste Ausschreibung werden als gesamter und auf volle Minuten gerundeter Zeitaufwand sowohl der in Tabelle 8-17 dargestellte Mittelwert von 239 Minuten als auch der Median von 160 Minuten zugrunde gelegt.

Tab. 8-17: Mittelwert und Median des Zeitaufwands der Ausschreibung 2004/2005

\begin{tabular}{|l|c|c|}
\hline & Mittelwert & Median \\
\hline Beschaffung & 31 Minuten & 15 Minuten \\
\hline Lesen und Verstehen & 39 Minuten & 30 Minuten \\
\hline Angebotsermittlung & 91 Minuten & 55 Minuten \\
\hline Ausfüllen & 78 Minuten & 60 Minuten \\
\hline Gesamt & 239 Minuten & 160 Minuten \\
\hline
\end{tabular}

Quelle: Eigene Berechnungen.

Für die monetäre Bewertung dieses Zeitaufwands findet der von den Landwirten als angemessen eingeschätzte Stundenlohn Anwendung, welcher in Form des auf ganze Euro gerundeten Mittelwerts von $17 €$ und des Medians von $15 €$ in Tabelle 8-18 abgebildet ist. 
Tab. 8-18: Mittelwert und Median des als angemessen eingeschätzten Stundenlohns für die Angebotsabgabe (2004/2005)

\begin{tabular}{|l|c|c|}
\hline & Mittelwert & Median \\
\hline Stundenlohn & $17 €$ & $15 €$ \\
\hline
\end{tabular}

Quelle: Eigene Berechnungen.

Auf der Grundlage dieser Ergebnisse werden im Folgenden die eingangs erläuterten zwei unterschiedlichen Berechnungen der Transaktionskosten für die Ausschreibung 2004/2005 vorgenommen. ${ }^{157}$

\section{Mittelwert des Zeitaufwands und Mittelwert des Stundenlohns}

Die erste Berechnung der Transaktionskosten erfolgt, indem der Mittelwert des Zeitaufwands in Höhe von 239 Minuten mit dem Mittelwert des seitens der Landwirte als angemessen eingeschätzten Stundenlohns in Höhe von $17 €$ in Beziehung gesetzt wird.

Daraus ergeben sich Transaktionskosten je Betrieb in Höhe von 67,72 €. Insgesamt haben 24 Landwirte eine Angabe zu den von ihnen angebotenen Schlägen gemacht, was zu Transaktionskosten in Höhe von 1.625,28 € führt. Hieraus lassen sich für die von den relevanten Landwirten angebotenen 114 Schläge Transaktionskosten pro Schlag in Höhe von 14,26 € ermitteln. Für alle 199 Schläge, die von insgesamt 38 Landwirten im Rahmen der Ausschreibung fristgerecht und formal korrekt angeboten wurden, folgen daraus gesamte Transaktionskosten der Angebotserstellung und abgabe in Höhe von 2.837,74 € (siehe Tabelle 8-21).

\section{Median des Zeitaufwands und Median des Stundenlohns}

Als zweite Ermittlung der Transaktionskosten werden der Median des Zeitaufwands und der Median des von den Landwirten als angemessen eingeschätzten Stundenlohns herangezogen, so dass nun 160 Minuten Zeitaufwand und $15 €$ Stundenlohn in Beziehung gesetzt werden.

\footnotetext{
${ }^{157}$ In diesem Zusammenhang ist für die Ausschreibungsrunde 2004/2005 auf eine erst nach Abschluss der ersten Befragung erkannte und maßgeblich durch die Relevanz zweier unterschiedlicher Befragungen begründete Ungenauigkeit hinzuweisen, denn es wurde im Rahmen der ersten Befragung nicht nach der Anzahl der jeweils angebotenen Schläge gefragt. Für die Berechnungen kann aber auf die Angaben der zweiten Befragung zurückgegriffen werden, da in beiden Befragungen ausschließlich die Landwirte relevant waren, die in der ersten Ausschreibung ein Angebot abgegeben haben und demzufolge eine weitestgehende Übereinstimmung der befragten Landwirte gegeben ist.
} 
Daraus ergeben sich für einen durchschnittlichen Betrieb Transaktionskosten in Höhe von $40 €$, für alle 24 in der Befragung relevanten Landwirte gesamte Transaktionskosten in Höhe von $960 €$ und Transaktionskosten je Schlag in Höhe von 8,42 €. Für alle im Rahmen der Ausschreibung angebotenen 199 Schläge führt dies zu gesamten Transaktionskosten der Landwirte von 1.675,58 € (siehe Tabelle 822).

\subsubsection{Transaktionskosten der Ausschreibung 2006}

Analog zur Berechnung der Transaktionskosten innerhalb des Ausschreibungsverfahrens 2004/2005 werden nachfolgend im Hinblick auf den in der zweiten Ausschreibung benötigten gesamten Zeitaufwand die auf ganze Minuten gerundeten Werte des Mittelwerts in Höhe von 165 Minuten und des Medians in Höhe von 140 Minuten zugrunde gelegt. Die relevanten Werte sind in Tabelle 8-19 dargestellt, wobei zu berücksichtigen ist, dass bedingt durch das spezifische Vorgehen im Rahmen der beschränkten Ausschreibung 2006 eine Beschaffung der Ausschreibungsunterlagen hier nicht einzubeziehen ist.

Tab. 8-19: Mittelwert und Median des Zeitaufwands der Ausschreibung 2006

\begin{tabular}{|l|c|c|}
\hline & Mittelwert & Median \\
\hline Beschaffung & -- & -- \\
\hline Lesen und Verstehen & 33 Minuten & 30 Minuten \\
\hline Angebotsermittlung & 68 Minuten & 50 Minuten \\
\hline Ausfüllen & 64 Minuten & 60 Minuten \\
\hline Gesamt & $\mathbf{1 6 5}$ Minuten & $\mathbf{1 4 0}$ Minuten \\
\hline
\end{tabular}

Quelle: Eigene Berechnungen.

Zur monetären Bewertung des Zeitaufwands werden die in Tabelle 8-20 dargestellten und auf ganze Euro gerundeten individuellen Angaben der Landwirte zum als angemessen eingeschätzten Stundenlohn in Form des Mittelwerts in Höhe von $21 €$ und des Medians von $20 €$ herangezogen.

Tab. 8-20: Mittelwert und Median des als angemessen eingeschätzten Stundenlohns für die Angebotsabgabe 2006

\begin{tabular}{|l|c|c|}
\hline & Mittelwert & Median \\
\hline Stundenlohn & $21 €$ & $20 €$ \\
\hline
\end{tabular}

Quelle: Eigene Berechnungen. 
Demzufolge ergeben sich erneut die folgenden zwei Wege zur Berechnung der Transaktionskosten.

\section{Mittelwert des Zeitaufwands und Mittelwert des Stundenlohns}

Die erste Berechnung erfolgt unter Einbeziehung der Mittelwerte des Zeitaufwands der Angebotsabgabe und des seitens der Landwirte dafür als angemessen eingeschätzten Stundenlohns.

Daraus folgen bei einem Zeitaufwand von 165 Minuten und einem Stundenlohn von $21 €$ Transaktionskosten für einen sowohl innerhalb der Ausschreibung als auch der Befragung relevanten Betrieb in Höhe von 57,75 €. Im Ganzen haben alle 25 Landwirte eine Angabe gemacht und 211 Schläge angeboten, so dass sich daraus gesamte Transaktionskosten für alle an der Befragung teilnehmenden Betriebe von 1.443,75 $€$ und entsprechende Transaktionskosten pro Schlag von 6,84 € ergeben. Unter Berücksichtigung aller 295 durch 30 Landwirte im Rahmen des Ausschreibungsverfahrens 2006 angebotenen Schläge führt dies zu dem Ergebnis, dass die Angebotsabgabe auf Seiten der Landwirte zu gesamten Transaktionskosten in Höhe von 2.017,80 € geführt hat (siehe Tabelle 8-21).

\section{Median des Zeitaufwands und Median des Stundenlohns}

Die zweite Variante der Transaktionskostenberechnung beruht darauf, sowohl hinsichtlich des Zeitaufwands als auch des Stundenlohns den im Rahmen der Befragungen ermittelten Median zugrunde zu legen.

Demzufolge ergeben sich bei einem Zeitaufwand von 140 Minuten und einem Stundenlohn in Höhe von $20 €$ für einen durchschnittlichen Betrieb Transaktionskosten der Angebotserstellung in Höhe von 46,67 $€$ und für alle 25 in der Befragung relevanten Landwirte gesamte Transaktionskosten in Höhe von 1.166,75 €. Von diesen 25 Landwirten wurden 211 Schläge angeboten, woraus sich pro Schlag Transaktionskosten in Höhe von 5,53€ ergeben. Für alle innerhalb der Ausschreibung angebotenen 295 Schläge entspricht dies gesamten Transaktionskosten im Umfang von 1.631,35 € (siehe Tabelle 8-22). 


\subsection{Zusammenfassende Diskussion}

Die Ermittlung und Bewertung der Transaktionskosten abschließend, werden die im Kontext dieser Arbeit maßgeblichen Aspekte zusammenfassend diskutiert. Dazu werden eingangs die Ergebnisse für beide Ausschreibungsrunden sowohl auf der Grundlage der Mittelwerte als auch der Mediane verglichen, wobei Tabelle 8-21 einführend die Mittelwerte zusammenfasst.

Tab. 8-21: Gegenüberstellung der Transaktionskosten für die Ausschreibungen 2004/2005 und 2006 auf Grundlage der Mittelwerte

\begin{tabular}{|l|c|c|}
\hline & $\begin{array}{c}\text { Ausschreibung } \\
2004 / 2005\end{array}$ & $\begin{array}{c}\text { Ausschreibung } \\
2006\end{array}$ \\
\hline Gesamter Zeitaufwand & 239 Minuten & 165 Minuten \\
\hline Stundenlohn & $17,00 €$ & $21,00 €$ \\
\hline Transaktionskosten je Betrieb & $67,72 €$ & $57,75 €$ \\
\hline Transaktionskosten je Schlag & $14,26 €$ & $6,84 €$ \\
\hline Gesamte Transaktionskosten & $2.837,74 €$ & $2.017,80 €$ \\
\hline
\end{tabular}

Quelle: Eigene Berechnungen.

Als Vergleich dazu erfolgt die zusammenfassende Gegenüberstellung der sich auf Grundlage der Mediane herausgebildeten Ergebnisse der Transaktionskostenermittlung in Tabelle 8-22.

Tab. 8-22: Gegenüberstellung der Transaktionskosten für die Ausschreibungen 2004/2005 und 2006 auf Grundlage der Mediane

\begin{tabular}{|l|c|c|}
\hline & $\begin{array}{c}\text { Ausschreibung } \\
2004 / 2005\end{array}$ & $\begin{array}{c}\text { Ausschreibung } \\
2006\end{array}$ \\
\hline Gesamter Zeitaufwand & 160 Minuten & 140 Minuten \\
\hline Stundenlohn & $15,00 €$ & $20,00 €$ \\
\hline Transaktionskosten je Betrieb & $40,00 €$ & $46,67 €$ \\
\hline Transaktionskosten je Schlag & $8,42 €$ & $5,53 €$ \\
\hline Gesamte Transaktionskosten & $1.675,58 €$ & $1.631,35 €$ \\
\hline
\end{tabular}

Quelle: Eigene Berechnungen.

Im Vergleich der beiden Ausschreibungsrunden zeigt sich zum einen, dass bei der Berechnung auf der Grundlage der Mediane durchweg geringere Werte ermittelt wurden. Zum anderen sind, bis auf die mittels der Mediane berechneten Transaktionskosten je Betrieb, alle Transaktionskosten für die Ausschreibungsrunde 2006 geringer als die im Rahmen der ersten Ausschreibung. 
Hierbei ist zu berücksichtigen, dass innerhalb der Ausschreibung im Jahr 2006 seitens der Landwirte keine Beschaffung der Unterlagen relevant war und demzufolge der am geringsten zeitaufwändige Arbeitsschritt entfallen ist, was unmittelbar einen Teil des Rückgangs des gesamten Zeitaufwands erklärt. Demgegenüber ist anzumerken, dass im Rahmen dieses zweiten Ausschreibungsverfahrens seitens der Landwirte ein insgesamt höherer Stundenlohn als angemessen eingeschätzt und folglich in die Berechnungen einbezogen wurde. Es tritt so ein gegenläufiger Effekt auf, welcher einen Anstieg der Transaktionskosten mit sich bringt.

Bedingt durch die schlagbezogene Angebotsabgabe und eine unterschiedliche Anzahl angebotener Schläge in beiden Ausschreibungsrunden sind letztlich für eine Bewertung der Veränderung der Transaktionskosten von der ersten zur zweiten Ausschreibung weder die Transaktionskosten je Betrieb noch die gesamten Transaktionskosten ausschlaggebend, sondern vielmehr die Transaktionskosten pro angebotenem Schlag. Diese haben sich von der ersten zur zweiten Ausschreibung auf der Grundlage der Mittelwerte von 14,26 € auf 6,84 $€$ und bei Zugrundelegen der Mediane von 8,42 € auf 5,53€ verringert.

Über eine ausschließliche Kostenbetrachtung hinaus werden nachfolgend die in Kapitel Sieben ermittelten Angebotspreise einbezogen und das schlagbezogene Verhältnis der Transaktionskosten zu den Angebotssummen der eingegangenen Angebote diskutiert. Die entsprechenden Anteile der Transaktionskosten sind für beide Ausschreibungen sowohl auf der Grundlage der Mittelwerte als auch der Mediane jeweils für die ökologischen Güter Grünland I, II und III in Tabelle 8-23 gegenübergestellt.

Tab. 8-23: Anteile der Transaktionskosten je Schlag an den Angebotspreisen der eingegangenen Angebote für die Ausschreibungen 2004/2005 und 2006 auf Grundlage der Mittelwerte und der Mediane

\begin{tabular}{|l|c|c|c|c|}
\hline \multirow{2}{*}{} & \multicolumn{2}{|c|}{ Ausschreibung 2004/2005 } & \multicolumn{2}{c|}{ Ausschreibung 2006 } \\
\cline { 2 - 5 } & Mittelwert & Median & Mittelwert & Median \\
\hline Grünland I & $9,1 \%$ & $7,2 \%$ & $4,5 \%$ & $5,6 \%$ \\
\hline Grünland II & $6,5 \%$ & $4,6 \%$ & $3,3 \%$ & $3,6 \%$ \\
\hline Grünland III & $4,0 \%$ & $5,9 \%$ & $2,1 \%$ & $1,7 \%$ \\
\hline
\end{tabular}

Quelle: Eigene Berechnungen. 
Die Datengrundlage der Berechnungen bilden für die Angebotssummen die in Kapitel Sieben erläuterten Ausschreibungsergebnisse. Die Transaktionskosten beziehen sich für die erste Ausschreibung auf die Tabelle 8-21 und für die zweite Ausschreibung auf die Tabelle 8-22.

Die Ergebnisse zeigen für beide Ausschreibungen und alle Abstufungen der ökologischen Güter einen relativ geringen Anteil der Transaktionskosten. Im Hinblick auf die hier relevante Veränderung des Verhältnisses der schlagbezogenen Transaktionskosten zu den Angebotssummen ist durchweg eine Verringerung der Relevanz der Transaktionskosten von der ersten zur zweiten Ausschreibungsrunde zu erkennen, was die transaktionskostenmindernden Effekte einer wiederholten (identischen) Ausschreibung verdeutlicht.

Unter Berücksichtigung der in den obigen Ausführungen verdeutlichten Abhängigkeit der Höhe der Transaktionskosten von dem gewählten methodischen Vorgehen und entsprechenden Einflussmöglichkeiten ist anzumerken, dass der Median im Vergleich zum Mittelwert grundsätzlich als robuster gegenüber Ausreißern anzusehen ist. Demzufolge wird hier die Einschätzung vertreten, dass die auf Grundlage der Mediane berechneten Ergebnisse insgesamt als aussagekräftiger anzusehen sind und für über diese Arbeit hinausgehende Betrachtungen herangezogen werden sollten.

Bei der Interpretation der Transaktionskosten sind an dieser Stelle zudem die zwangsläufig mit der gewählten empirischen Vorgehensweise und grundsätzlich mit einer Transaktionskostenermittlung verbundenen Ungenauigkeiten und Abgrenzungsschwierigkeiten zu berücksichtigen.

Demzufolge soll und kann in diesem Kontext nicht der Anspruch erhoben werden, dass die ermittelten Transaktionskosten exakte Quantifizierungen innerhalb der Modellausschreibungen darstellen. Vielmehr sollen sie als Richtwerte in die Diskussion eingebracht werden, um eine relative Bewertung von Ausschreibungen und Prämienzahlungen zu ermöglichen und darüber hinaus die Schwierigkeiten der Ermittlung und der Aussagekraft von Transaktionskosten zu verdeutlichen.

Diesbezüglich hat sich gezeigt, dass in der Tendenz für die Landwirte keine höheren Transaktionskosten der Angebotsabgabe innerhalb eines Ausschreibungsverfahrens gegenüber der Antragstellung für Prämienzahlungen in bestehenden Agrarumweltprogrammen zu erwarten sind. Dies unterstreicht eine positive 
Bewertung von Ausschreibungen, da nicht zu erwarten ist, dass praktische Effizienzvorteile reduziert werden oder höhere Transaktionskosten einer Teilnahme der Landwirte entgegenstehen.

Zur Bewertung der Möglichkeiten einer Einbeziehung von Transaktionskosten gemäß der ELER-Verordnung zeigen sich anhand der Befragungsergebnisse grundlegende Probleme.

Die je nach Berechnungsgrundlage unterschiedlichen Transaktionskosten und der individuell sehr differenzierte Zeitaufwand der Angebotserstellung unterstützen letztlich unabhängig von der tatsächlichen Höhe der Transaktionskosten - die Einschätzung, dass die Ermittlung eines für alle Landwirte einheitlichen Werts nicht möglicht ist und mit den analogen Problemen einer einheitlichen Prämie einhergeht. Infolgedessen ist eine ökonomisch vertretbare Implementierung von Transaktionskosten entsprechend des Artikels 39 der ELER-Verordnung in eine Einheitsprämie als nicht praktikabel einzuschätzen.

Darüber hinaus stellt sich in Verbindung mit der eingangs erläuterten Problematik, dass innerhalb des Artikels 39 der ELER-Verordnung keine Definition der Transaktionskosten gegeben wird, die Frage, ob letztlich politisch eine Ermittlung und eine darauf aufbauende Berücksichtigung der Transaktionskosten erwünscht ist. Dies ist zu bezweifeln und vielmehr ist zu vermuten, dass durch die suggerierte Einbeziehung von Transaktionskosten eine weitere Rechtfertigung unspezifischer und intransparenter Zahlungen an Landwirte unter einer neuen Bezeichnung als der bisherigen Anreizkomponente von $20 \%$ angestrebt wird.

Überwunden werden kann dieses Problem durch die Nutzung von Ausschreibungsverfahren, da hierbei keine staatlich fixierten Prämien (und folglich auch keine Transaktionskosten) vorgegeben werden müssen, sondern die individuellen Angebotspreise relevant sind. Es kann demzufolge eine Kalkulation der Transaktionskosten in den Angebotspreis erfolgen, woraufhin eine angemessene Honorierung der Landwirte zu erwarten ist.

Neben diesen Überlegungen stellt sich abschließend die in den obigen Ausführungen bereits angesprochene Frage der Relevanz der ermittelten Transaktionskosten, da innerhalb der Ausschreibung 2004/2005 der Zeitaufwand lediglich von 45,8 \% und 
im Zuge der Ausschreibung 2006 mit 48,0 \% ebenfalls von weniger als der Hälfte der befragten Landwirte einkalkuliert wurde.

Zur Bewertung dieser Ergebnisse sind zwei Herangehensweisen denkbar. Einerseits kann argumentiert werden, dass die Relevanz der Transaktionskosten zu vernachlässigen ist, da sie von weniger als der Hälfte der Landwirte berücksichtigt und somit lediglich in diesem Umfang bei der Zuschlagserteilung über die Preise entscheidungsrelevant wurden. Eine andere Argumentation kann dahingehend vorgenommen werden, dass die Transaktionskosten gerade durch die überwiegend nicht erfolgte Kalkulation und Einbeziehung in die Angebotspreise relevant sind und dies ihre Berücksichtigung notwendig macht.

Für letztere Argumentation spricht, dass unabhängig von ihrer spezifischen Höhe bei der Angebotserstellung für die Landwirte grundsätzlich Transaktionskosten entsprechend der hier vorgenommenen Definition entstanden sind. Folglich sind die Transaktionskosten für die Landwirte existent und für den Fall, dass sie nicht in die Preise einkalkuliert werden, erfahren diese Landwirte eine implizite finanzielle Einbuße.

Weiterhin muss auf die für die Landwirte besondere Situation eines Ausschreibungsverfahrens hingewiesen werden, da hierbei nicht wie bislang üblich eine einheitliche und alles abdeckende Prämie gezahlt wird, sondern die Landwirte eigenverantwortlich den für sie relevanten Preis kalkulieren und im Zuge dessen entscheiden, welche Kosten sie einbeziehen. Dabei ist grundsätzlich die Situation gegeben, dass mit steigenden Angebotspreisen die Wahrscheinlichkeit einer Zuschlagserteilung sinkt und für die Landwirte der Trade-off zwischen Berücksichtigung sowohl aller Kosten und eines Gewinnzuschlags einerseits und andererseits der Gefahr zu hoher Angebotspreise, mit der Folge für die relevante Fläche keine Zahlung realisieren zu können, gelöst werden muss.

Zusammenfassend wird deutlich, dass keine einheitliche und für alle Landwirte gültige Bewertung der Relevanz von Transaktionskosten möglich ist. Vielmehr zeigt sich, aufgedeckt durch den Einsatz von Ausschreibungsverfahren, eine spezifische einzelwirtschaftliche Entscheidungsfindung und Berücksichtigung der Transaktionskosten.

Demzufolge kann an dieser Stelle keine objektive Wertung vorgenommen werden und es gilt erneut sowohl auf die informationsaufdeckende Funktion von 
Ausschreibungen als auch auf die Vorteilhaftigkeit marktwirtschaftlicher Strukturen zu verweisen, welche auf der Grundlage der Preiskalkulation eine entsprechende individuelle Einbeziehung und Entscheidungsrelevanz der Transaktionskosten sicherstellen. 


\section{Weiterer Forschungsbedarf}

Abschließend wird über die vorliegende Arbeit hinausgehender Forschungsbedarf aufgezeigt, wobei der praxisbezogenen Herangehensweise folgend hier ausschließlich praktische Fragestellungen aufgegriffen werden. So werden im Folgenden fünf thematische Abgrenzungen vorgenommen und der sich daraus ergebende Forschungsbedarf wird anhand einiger zentraler Gesichtspunkte skizziert. Darüber hinaus werden erste Verweise auf im Rahmen zukünftiger Untersuchungen zu berücksichtigende Ansätze und in dieser Arbeit gewonnene Erkenntnisse gegeben.

\section{Weitere praktische Erprobungen von Ausschreibungen}

Wie im Verlauf der Arbeit gezeigt wurde, konnten mit dem hier zugrunde gelegten spezifischen Ausschreibungsdesign alle Ziele eines Ausschreibungsverfahrens erfüllt werden.

Nichtsdestoweniger ist deutlich geworden, dass es keine für alle Anwendungsbereiche der Ausschreibung von Umweltleistungen einheitliche und eine erfolgreiche Umsetzung sicherstellende Ausgestaltung geben kann. Somit besteht über diese spezifische Herangehensweise und weitere bereits erfolgte praktische Erprobungen von Ausschreibungen hinaus ein ergänzender Forschungsbedarf. Im Zuge dessen sind vor allem die Einflüsse unterschiedlicher Umweltleistungen und Verwaltungsstrukturen sowie spezifischer regionaler Abgrenzungen auf die Praktikabilität und die Zielerreichung von Ausschreibungsverfahren zu untersuchen. Hierbei muss abgewogen werden, in welchen Konstellationen die alleinige Nutzung eines Ausschreibungsverfahrens nicht erfolgen kann und letztlich eine Beibehaltung der einheitlichen Honorierung oder die Kombination einer Prämienzahlung mit einem Ausschreibungsverfahren angemessen ist.

Darüber hinaus ist ein weiterer Forschungsbedarf hinsichtlich des langfristigen praktischen Vergleichs von sich lediglich in der Wahl des Vertragsabschlusses unterscheidenden Ausschreibungen vorhanden. Hierzu sind die preisdiskriminierende Ausschreibung und die Einheitspreis-Ausschreibung heranzuziehen und im Hinblick auf die zu erwartenden Auswirkungen der jeweiligen Zuschlagserteilung auf die langfristige Zielerreichung unter Berücksichtigung des Bieterverhaltens der Landwirte und ihrer Informationsverarbeitung zu untersuchen. 


\section{Entwicklung und Implementierung von Umweltindikatoren}

Wie andere umwelt- und agrarpolitische Fragestellungen erfordert auch die Förderung und Erhaltung der biologischen Vielfalt die gemeinsame Betrachtung ökologischer und ökonomischer Aspekte. Nur so kann eine nachhaltige gesellschaftliche Legitimation staatlichen Handelns und eine auch dauerhaft erfolgreiche Implementierung institutioneller Neuerungen erreicht werden. Diese ebenfalls in Artikel 39 der ELER-Verordnung angesprochene Forderung einer Berücksichtigung von wirtschaftlichen und ökologischen Effizienzkriterien zeigt einen weitergehenden Forschungsbedarf auf.

Innerhalb von Ausschreibungsverfahren ist der zentrale Ansatzpunkt hierbei in der Ausgestaltung der Zuschlagserteilung und somit der einem Vertragsabschluss zugrunde liegenden Kriterien zu sehen. Zur Berücksichtigung entsprechender naturschutzfachlicher Anforderungen bietet sich - neben dem in dieser Arbeit verfolgten, und durch die Ausgestaltung des Forschungsprojekts vorgegebenen, Ansatz der Einordnung der Angebote in ökologische Qualitätsstufen - vor allem die Option einer Angebotsbewertung unter Rückgriff auf Umweltindikatoren.

Durch einen Umweltindikator wird die Möglichkeit eröffnet, neben dem Angebotspreis je Einheit ökologische und soziale Kriterien zu definieren, anhand derer die eingehenden Angebote bewertet werden. In der Regel werden hierbei für jedes Kriterium Punkte vergeben, so dass nach der Angebotsbewertung jede angebotene Fläche über einen Gesamtpunktwert verfügt und die Zuschlagserteilung beginnend mit dem höchsten Punktwert bis zur Ausschöpfung des Budgets oder anderer Vorgaben erfolgen kann. Je nach Gewichtung der Kriterien können zudem spezifische Schwerpunktsetzungen und Anpassungen der Ziele einer Ausschreibung vorgenommen werden.

Demnach ist ein entsprechender Forschungsbedarf auch innerhalb der europäischen Agrarumweltpolitik zu sehen, wobei sowohl die Entwicklung als auch die praktische Erprobung von Umweltindikatoren notwendig sind, um im Zuge dessen das Potential der Implementierung eines flexiblen und ökonomisch vertretbaren Vorgehens zur Verbindung ökologischer, sozialer und ökonomischer Kriterien abzuwägen.

Als praktische Grundlage dieser Erprobung können sowohl der innerhalb des Conservation Reserve Program in den USA implementierte Environmental Benefits Index als auch ein sich im Rahmen des BushTender Trial in Australien bewährter Umweltindikator dienen. 


\section{Operative Einbindung der Agrarverwaltung sowie Ermittlung und}

\section{Bewertung der Verwaltungstransaktionskosten}

Mit dem Ziel einer effizienten Implementierung von Ausschreibungsverfahren in die nationale und internationale Agrarumweltpolitik ist es notwendig, einen weiteren Schritt in die Praxis zu gehen. Dieser beinhaltet im Rahmen zukünftiger Erprobungen, neben der hier bereits vollzogenen praktischen Einbeziehung und Analyse der Landwirte, ebenfalls eine operative Einbindung der Agrarverwaltung in den Ablauf eines Ausschreibungsverfahrens. In diesem Fall können sowohl institutionelle Voraussetzungen der Einführung von Ausschreibungen in die Verwaltungspraxis als auch mögliche Vorbehalte der Agrarverwaltung und politischer Entscheidungsträger einbezogen werden.

Hinsichtlich der Ermittlung und Bewertung der Verwaltungstransaktionskosten besteht im Kontext von Agrarumweltprogrammen die Möglichkeit auf unterschiedliche Ansätze zurückzugreifen, wobei zudem eine kritische Würdigung des dort gewählten methodischen Vorgehens erfolgen kann.

Zur Sicherstellung eines praktischen Institutionenvergleichs im Hinblick auf die spezifische Verwaltungseinbindung und die damit verbundenen Transaktionskosten einer Einheitsprämie und eines Ausschreibungsverfahrens, sollte an dieser Stelle zudem ein bestehendes und hinsichtlich seiner ökologischen Ziele dem Ausschreibungsverfahren identisches bzw. ähnliches Agrarumweltprogramm als Referenz herangezogen werden.

\section{Umsetzung einer digitalen und internetbasierten Angebotsabgabe bzw.} Antragstellung

Die im Rahmen der Befragungen zur Ermittlung und Bewertung der Transaktionskosten in dieser Arbeit bereits einbezogenen Aspekte einer digitalen Antragstellung unter Nutzung des Internets haben einen darauf aufbauenden Forschungsbedarf erkennen lassen. Eine derartige Ausgestaltung umfasst den gesamten Prozess der Antragstellung, von der Möglichkeit sämtliche relevanten Formulare und Erläuterungen aus dem Internet herunterladen und unmittelbar ausfüllen zu können, bis hin zur digitalen Datenübermittlung beispielsweise per EMail. Diese Daten können anschließend von der Agrarverwaltung ebenfalls softwaregestützt bearbeitet und ausgewertet werden. Darüber hinaus besteht die Möglichkeit, nicht veränderte Daten aus vorherigen Antragstellungen übernehmen zu 
können, so dass insgesamt sowohl für die Landwirte als auch die Agrarverwaltung transaktionskostenmindernde Effekte zu erwarten sind.

Anhand der in Kapitel Acht der vorliegenden Arbeit präsentierten Befragungsergebnisse ist zu erkennen, dass sich in beiden Ausschreibungsrunden jeweils eine Mehrheit der Landwirte dafür offen gezeigt hat, zukünftig von einer entsprechenden Antragstellung bzw. Angebotsabgabe Gebrauch zu machen. Auf diesen ersten positiven Einschätzungen aufbauend ist ein zukünftiger Forschungsbedarf darin zu sehen, eine an der bestehenden Verwaltungspraxis orientierte und rechtssichere Ausgestaltung einer Online-Antragstellung unter Einbeziehung der Agrarverwaltung und der Landwirte praktisch zu erproben und mittelfristig zu implementieren.

Zudem bietet sich die Möglichkeit, die Entwicklung der Transaktionskosten und die mit einer Anpassung der Antragstellung verbundenen Einsparpotentiale zu bewerten. Im Zuge dessen kann die empirische Basis der Transaktionskostenökonomik erweitert und gleichermaßen ihre spezifische praktische Anwendbarkeit analysiert werden.

\section{Einbeziehung anderer Landbewirtschafter in Agrarumweltmaßnahmen}

Aufbauend auf der in Artikel 39 der ELER-Verordnung eingeräumten Möglichkeit, soweit dies zur Erreichung der Umweltziele gerechtfertigt ist, Zahlungen für Agrarumweltmaßnahmen auch anderen Landbewirtschaftern als Landwirten gewähren zu können, ergibt sich ein weitreichendes Entwicklungspotential der Agrarumweltpolitik. Grundsätzlich ist eine derartige Öffnung von Agrarumweltprogrammen positiv einzuschätzen, denn letztlich ist es für den Umwelterfolg unwesentlich, ob eine Maßnahme zum Schutz der Biodiversität oder ganz allgemein eine Umweltleistung von Landwirten oder anderen Landbewirtschaftern erbracht wird.

Im Hinblick auf die praktische Umsetzung und Ausgestaltung dieser Öffnung der Agrarumweltmaßnahmen zeigt sich jedoch umfassender Forschungsbedarf. Hierbei sind vor allem Fragen der rechtlichen und formalen Ausgestaltung einer Einbeziehung von anderen Landbewirtschaftern bis hin zu ihrer Finanzierung aus grundsätzlich für die Agrarumweltpolitik und demzufolge für Landwirte vorgesehenen Mitteln zu beantworten. Diesbezüglich gilt es zudem Akzeptanzfragen sowohl seitens landwirtschaftlicher Betriebe als auch ihrer Interessenvertreter zu 
untersuchen und die praktischen Möglichkeiten dieser potentiell viel versprechenden Weiterentwicklung der Agrarumweltpolitik auszuloten.

Darüber hinaus ist vor dem Hintergrund des Einsatzes von Ausschreibungsverfahren zu prüfen, welche Effekte durch eine Erweiterung des Teilnehmerkreises an Agrarumweltprogrammen beispielsweise hinsichtlich der damit verbundenen steigenden Konkurrenzsituation und im Zuge dessen zusätzlicher Einsatzmöglichkeiten von Ausschreibungen zu erwarten sind.

Neben diesen thematisch über die vorliegende Arbeit hinausgehenden Aspekten besteht ebenfalls weiterer Forschungsbedarf hinsichtlich hier bereits einbezogener Fragen.

So sind vor allem die dauerhafte Akzeptanz von Ausschreibungen, der Umgang mit möglichen Mitnahmeeffekten und das langfristige Bieterverhalten von Landwirten unter Berücksichtigung der Verarbeitung sich verändernder Informationen und möglicher Preisabsprachen zu untersuchen, um den Weg für eine umfassende Implementierung von vielseitigen, flexiblen und effizienten Ausschreibungen auch in die praktische europäische Agrarumweltpolitik zu bereiten. 


\section{Zusammenfassung und Fazit}

Diese Arbeit betrachtet einen ökonomischen Teilaspekt der im Zeitraum 2004 bis 2006 durchgeführten Umsetzung eines interdisziplinären Forschungsprojekts zur ergebnisorientierten Honorierung ökologischer Leistungen der Landwirtschaft auf Grünlandflächen. Als Modellregion dient der Landkreis Northeim in Südniedersachsen.

Der praktische Bezug der Forschungsarbeit, und im Zuge dessen ihre agrarpolitische Relevanz, ergibt sich vor allem aus zwei in Artikel 39 der ELER-Verordnung bestimmten Veränderungen der Honorierung von Landwirten in Agrarumweltprogrammen. Demzufolge können ab 2007 erstmals a) Zahlungen für Agrarumweltmaßnahmen über eine Ausschreibung anstelle einer einheitlichen Prämie erfolgen und b) Transaktionskosten als ein Kriterium zur Bemessung von Zahlungen an Landwirte fungieren.

Die Ziele der Untersuchung stellen hinsichtlich dieser Weiterentwicklung der Agrarumweltpolitik die Erprobung von Ausschreibungen und die Diskussion der Möglichkeiten einer Einbeziehung von Transaktionskosten in die Zahlungen an Landwirte dar. Des Weiteren wird der Frage nachgegangen, inwieweit der Analyserahmen der Transaktionskostenökonomik zur Untersuchung der spezifischen Transaktionsbeziehung zwischen Agrarverwaltung und Landwirten herangezogen werden kann.

\section{Somit lassen sich die im Kontext dieser praxisbezogenen Arbeit wesentlichen}

\section{Ziele wie folgt zusammenfassen:}

I. Die Übertragung auktionstheoretischer Grundlagen auf den Bereich der Ausschreibung von Umweltleistungen und die Ableitung eines spezifischen Ausschreibungsdesigns für ökologische Leistungen der Landwirtschaft.

II. Die Anwendung des Analyserahmens der Transaktionskostenökonomik auf die Transaktionsbeziehung innerhalb des Ausschreibungsverfahrens.

III. Die praktische Erprobung von zwei aufeinander folgenden Ausschreibungen sowohl im Hinblick auf die sich einstellenden Angebotspreise als auch Fragen der Preiskalkulation und des Bieterverhaltens der Landwirte.

IV. Die Ermittlung und Bewertung der seitens der Landwirte mit einer Angebotserstellung verbundenen Transaktionskosten. 
Die Arbeit beginnt in Kapitel Eins mit der Einordnung der Untersuchung in den Kontext der aktuellen Agrarumweltpolitik und dem Aufzeigen des sich daraus hinsichtlich eines effizienten Einsatzes öffentlicher Finanzmittel durch Ausschreibungsverfahren und der Fokussierung auf die Transaktionskosten der Landwirte ergebenden Forschungsbedarfs. Hier wird zudem der multifunktionale Charakter der landwirtschaftlichen Produktion herausgestellt und unter Rückgriff auf die Anforderungen der Biodiversitätskonvention die ökologische Zielsetzung des Forschungsprojekts in Form des Erhalts und der Förderung pflanzlicher Artenvielfalt auf landwirtschaftlich genutzten Flächen aufgezeigt.

Kapitel Zwei führt die agrarumweltpolitischen Rahmenbedingungen dieser Arbeit ein. Hierbei werden die Grundlagen von Agrarumweltprogrammen betrachtet und ihre Entwicklung beginnend mit der EU-Agrarreform aus dem Jahr 1992 skizziert. Darauf aufbauend erfolgt eine kritische Würdigung der aktuellen Agrarumweltpolitik, welche sich auf die ungenügende Berücksichtigung von Bevölkerungspräferenzen und regionalem Expertenwissen, einer vornehmlich handlungsorientierten Ausrichtung und einem sowohl undifferenzierten als auch ineffizienten Einsatz öffentlicher Mittel durch die einheitliche Honorierung von Landwirten bezieht. Das Kapitel abschließend werden politische Ansätze zur Weiterentwicklung der internationalen und nationalen Agrarumweltpolitik einbezogen, wobei mit der Perspektive 2007 bis 2013 die ELER-Verordnung im Mittelpunkt steht.

Die Einordnung der vorliegenden Arbeit in den Gesamtkontext des Forschungsprojekts erfolgt im dritten Kapitel. Dazu werden mit den ökologischen Gütern, dem Regionalen Beirat Northeim, den regionalen Bevölkerungspräferenzen und dem Ausschreibungsverfahren die einzelnen Komponenten und die Zielsetzung des Honorierungssystems dargestellt. Zudem gibt das Kapitel durch die Charakterisierung der Modellregion und der Einbeziehung des zeitlichen Ablaufs der empirischen Arbeiten einen Überblick über die hier relevante Umsetzungsphase.

Den für die nachfolgende Analyse der Ausgestaltung von Ausschreibungen herangezogenen konzeptionellen Rahmen stellt die in Kapitel Vier betrachtete Transaktionskostenökonomik dar. Nach einem geschichtlichen Exkurs wird der 
Ansatz anhand der Verhaltensannahmen, der Dimensionen von Transaktionen und der Beherrschungs- und Überwachungsstrukturen eingeführt. Zudem werden grundlegende begriffliche Abgrenzungen vorgenommen und die in der Literatur vorherrschende Kritik am Konzept der Transaktionskostenökonomik aufgezeigt.

Im Anschluss wird im fünften Kapitel das für diese Arbeit zentrale Instrument einer Ausschreibung für Umweltleistungen einbezogen. $\mathrm{Zu}$ Beginn werden die auktionstheoretischen Grundlagen dargelegt und die in der Regel unterschiedenen Auktionsformen für Eingut- und Mehrgüterauktionen betrachtet. Unter Rückgriff auf diese Standardauktionsformen wendet sich die Analyse dem praktischen Einsatz von Ausschreibungen für Umweltleistungen zu. Nach einer Einführung in die entsprechenden Grundlagen werden die aus Sicht der Vergabestelle mit dem Einsatz von Ausschreibungsverfahren verfolgten Ziele der Kostenminimierung bzw. Nutzenmaximierung, des effizienten Einsatzes öffentlicher Finanzmittel und der Informationsgewinnung über die Kosten der Landwirte abgeleitet.

Darauf aufbauend erfolgt eine Diskussion der Übertragbarkeit der Standardauktionen auf den Bereich der Ausschreibung von Umweltleistungen. Im Ergebnis zeigt sich, dass für einen praktischen Einsatz die Niedrigstpreis-Ausschreibung als am besten geeignet anzusehen ist.

Jedoch greift diese Betrachtung von Eingutausschreibungen hier zu kurz, da innerhalb eines Ausschreibungsverfahrens für ökologische Leistungen der Landwirtschaft in der Regel mehrere Güter gleichzeitig ausgeschrieben werden und mitunter eine Vielzahl von Landwirten einen Zuschlag erhalten kann. Dies führt zu dem Ergebnis, dass ausschließlich Mehrgüterausschreibungen mit einer in jeder Ausschreibungsrunde einmaligen verdeckten Angebotsabgabe als geeignet einzuschätzen sind. Hinsichtlich der Zuschlagserteilung ist mit dem Ziel einer wiederholten Ausschreibung die preisdiskriminierende Ausschreibung einer Einheitspreis-Ausschreibung vorzuziehen. Daraus folgt, dass für die weiteren praktischen Untersuchungen eine als simultane Mehrgüterausschreibung mit PreisMengen-Angeboten und einer preisdiskriminierenden Zuschlagserteilung zu charakterisierende Ausschreibung Anwendung findet, wobei die regionale Abgrenzung einer einheitlichen Ausschlussgrenze mit einer Budgetrestriktion entspricht. 
Zum Abschluss des Kapitels werden mit dem Conservation Reserve Program, dem BushTender Trial und den Modellauschreibungen in Nordrhein-Westfalen drei bereits in der Praxis erprobte und sich in ihrer konzeptionellen Ausgestaltung grundlegend unterscheidende Ausschreibungsverfahren für Umweltleistungen einbezogen und der Stand der Forschung umrissen.

Das sechste Kapitel beinhaltet die transaktionskostenökonomische Analyse von Ausschreibungen für ökologische Leistungen der Landwirtschaft. Unter Berücksichtigung der Akteure (Agrarverwaltung und Landwirte) und der entsprechenden Vertragsbeziehung werden die für die Transaktionskostenökonomik elementaren Verhaltensannahmen und die Dimensionen von Transaktionen untersucht sowie eine effiziente Überwachung von Ausschreibungen diskutiert, wobei die Notwendigkeit einer über den Zeitpunkt der Zuschlagserteilung hinausgehenden Betrachtung verdeutlicht wird.

Hierbei zeigt sich, dass die einer Ausschreibung zugrunde liegende Transaktionsbeziehung durch die Eingebundenheit einer staatlichen Institution auf der Nachfrageseite von der traditionell innerhalb der Transaktionskostenökonomik thematisierten Vertragsbeziehung abweicht. Dies ist insbesondere dadurch zu begründen, dass auf Seiten der Agrarverwaltung keine praktischen Möglichkeiten für Opportunismus gegeben sind und demzufolge von einer vertragskonformen und Rechtssicherheit gebietenden Abwicklung durch die Vergabestelle ausgegangen werden kann.

Die praktische Relevanz der fundamentalen Transformation ist bedingt durch die hohe Faktorspezifität und entsprechend getätigte Investitionen im Rahmen der Ausgestaltung der Zuschlagserteilung in einer wiederholten Ausschreibung sowohl auf Seiten der Agrarverwaltung als auch seitens der Landwirte als stark ausgeprägt anzusehen.

Um diesen Besonderheiten Rechnung zu tragen, wird abschließend das in Kapitel Fünf gewählte Ausschreibungsdesign herangezogen, aus Sicht der Transaktionskostenökonomik analysiert und daraufhin die „einseitige Marktkontrolle“ als Erweiterung des Analyserahmens abgeleitet.

Den empirischen Teil der Arbeit beginnt Kapitel Sieben mit der ausführlichen Betrachtung der konzeptionellen Ausgestaltung und der Ergebnisse der 
Ausschreibungsrunden 2004/2005 und 2006. Im Zuge dessen wird für beide Ausschreibungen eine identische Herangehensweise gewählt und eingangs das formale und zeitliche Vorgehen erläutert. Hinsichtlich der Ausschreibungsergebnisse wird jeweils maßgeblich auf die Angebotspreise für die drei Qualitätsabstufungen der ökologischen Güter eingegangen und sie werden zunächst separat und im Anschluss zusammenfassend analysiert. Zudem erfolgt für beide Ausschreibungen die Einbeziehung der Ergebnisse schriftlicher Befragungen der teilnehmenden Landwirte zur Beantwortung von Fragen der Preiskalkulation und des Bieterverhaltens. Das Kapitel abschließend werden zusammenfassende Diskussionen der zentralen Ausschreibungs- und Befragungsergebnisse beider Ausschreibungen vorgenommen und ihre Veränderungen von der ersten zur zweiten Ausschreibungsrunde aufgezeigt.

Bei der Betrachtung der Angebotspreise wird deutlich, dass es bei allen ökologischen Leistungen in beiden Ausschreibungsrunden $\mathrm{zu}$ den theoretisch erwarteten differenzierten Angebotspreisen und mit einem höheren ökologischen Nutzen auch zu einem höheren Preisniveau gekommen ist. Daraus kann gefolgert werden, dass sich die Landwirte in der Modellregion mit individuell verschiedenen Kostenstrukturen und Produktionsbedingungen konfrontiert gesehen haben und in beiden Ausschreibungsrunden eine - zumindest teilweise - Orientierung daran stattgefunden hat, was das praktische Potential für einen effizienteren Einsatz knapper finanzieller Mittel durch Ausschreibungen im Vergleich zu einer einheitlichen Prämie verdeutlicht. Diese Vorteile unterstreichen im Rahmen der Arbeit durchgeführte Vergleiche der Ausschreibungsergebnisse mit aus ökologischer Sicht vergleichbaren Prämienzahlungen in bestehenden Agrarumweltprogrammen, wobei je nach Berechnungsgrundlage Effizienzvorteile von bis zu 26,2 \% in der ersten und bis zu 36,3 \% in der zweiten Ausschreibung realisiert werden konnten.

Im Hinblick auf die Ausprägung von Preisabsprachen und einer ausschreibungsrelevanten Kommunikation der Landwirte wird gezeigt, dass es in beiden Ausschreibungsrunden faktisch zu keinen sich negativ auf die praktische Zielerreichung auswirkenden Einflüssen gekommen ist und den Ausschreibungen eine ausreichende Konkurrenzsituation zugrunde gelegen hat.

Die Antworten zu einer über die Kostendeckung hinausgehenden Kalkulation der Angebotspreise verdeutlichen, dass die mit der Angebotserstellung verbundenen privaten Transaktionskosten der Landwirte und ein Gewinnzuschlag bislang lediglich 
in einem geringen Umfang Eingang gefunden haben. Dies ist sowohl auf die bisherige, einer eigenverantwortlichen Preiskalkulation entgegenstehende, einheitliche Honorierung von Landwirten als auch die spezifische neue Situation in einem Ausschreibungsverfahren zurückzuführen, da mit einem steigenden Angebotspreis in der Regel die Wahrscheinlichkeit einer Zuschlagserteilung abnimmt.

Im achten Kapitel werden die als bewerteter Zeitaufwand der Angebotserstellung definierten Transaktionskosten der Landwirte einbezogen. Nach dem Aufzeigen des diesbezüglich noch grundlegenden Forschungsbedarfs, erfolgt die Definition der relevanten Transaktionskosten und die Einführung des methodischen Vorgehens. Auf der Grundlage von drei schriftlichen Befragungen wird für beide Ausschreibungsrunden der zur Angebotserstellung benötigte Zeitaufwand ermittelt. Die monetäre Bewertung dieses Zeitaufwands, und somit die Ermittlung der Transaktionskosten, erfolgt auf der Basis des seitens der Landwirte für die Angebotserstellung als angemessen eingeschätzten Stundenlohns.

Die Ergebnisse zeigen, dass es in beiden Ausschreibungsrunden zu individuell sehr unterschiedlichen Beträgen und einer ebenfalls uneinheitlichen Relevanz der Transaktionskosten gekommen ist, wobei in der Tendenz ein leichter Rückgang von der ersten zur zweiten Ausschreibung zu erkennen ist. Zudem ist auf der Grundlage von Einschätzungen der Landwirte im Rahmen von Ausschreibungsverfahren kein höherer Zeitaufwand als in Agrarumweltprogrammen mit einer einheitlichen Prämienzahlung zu erwarten.

Eine über diese Kostenbetrachtung hinausgehende Analyse kommt je nach Berechnungsgrundlage und betrachtetem ökologischen Gut zu dem Ergebnis, dass der Anteil der Transaktionskosten je Schlag an den Angebotssummen der eingegangenen Angebote in der ersten Ausschreibung zwischen 4,0\% und 9,1\% liegt. In der zweiten Ausschreibung sind sowohl transaktionskostenmindernde Effekte als auch eine weiter abnehmende Relevanz zu erkennen und dieser Anteil der Transaktionskosten beträgt nunmehr zwischen 1,7 \% und 5,6 \%.

Die Arbeit schließt in Kapitel Neun mit dem Aufzeigen weitergehenden praktischen Forschungsbedarfs. Im Zuge dessen werden Fragen der ergänzenden Erprobungen von Ausschreibungen für Umweltleistungen, der operativen Einbindung der 
Agrarverwaltung, der Bewertung entsprechender Verwaltungstransaktionskosten, der Implementierung von Umweltindikatoren und einer transaktionskostenmindernden Ausgestaltung der Angebotsabgabe thematisiert. Darüber hinaus wird der Aspekt einer Erweiterung von Agrarumweltmaßnahmen auf andere Landbewirtschafter als Landwirte einbezogen.

Zusammenfassend zeigt sich im Hinblick auf die zentrale praktische Fragestellung der Untersuchung, dass mit dem im Rahmen dieser Arbeit gewählten Ausschreibungsdesign alle praktischen Ziele (Kostenminimierung, Effizienz und Informationsgewinnung) erfüllt werden konnten und seine Tragfähigkeit für eine Implementierung in die Agrarumweltpolitik bewiesen ist.

Darüber hinaus machen die Ergebnisse der Transaktionskostenermittlung deutlich, dass in der Praxis für die Transaktionskosten kein einheitlicher Wert existiert und eine staatliche Vorgabe entsprechend des Artikels 39 der ELER-Verordnung mit den analogen Kritikpunkten einer Einheitsprämie verbunden ist. Überwunden werden können diese Probleme durch die Nutzung eines Ausschreibungsverfahrens. Hierbei muss keine Transaktionskostenhöhe vorgegeben werden, sondern es kann eine Einbeziehung der individuellen Transaktionskosten in den Angebotspreis und demzufolge eine angemessene Honorierung erfolgen.

Mit dem Ziel einer vergleichenden Bewertung von Ausschreibungen und Prämienzahlungen zur Honorierung von Umweltleistungen lassen sich demzufolge auch hinsichtlich dieser spezifischen Umsetzung der ELER-Verordnung Vorteile von Ausschreibungen erkennen. 


\section{Literaturverzeichnis}

Alchian, A. A. (1950): Uncertainty, Evolution, and Economic Theory, in: Journal of Political Economy, 58, S. 211-221.

Alchian, A. A. (1965): Some Economics of Property Rights, in: Alchian, A. A. (Hrsg.): Economic Forces at Work. Indianapolis, S. 127-149.

Alchian, A. A. (1969): Information Costs, Pricing and Resource Unemployment, in: Western Economic Journal, 7, S. 109-128.

Alchian, A. A. und Demsetz, H. (1972): Production, Information Costs and Economic Organisation, in: American Economic Review, 62, S. 777-795.

Alchian, A. A. und Demsetz, H. (1973): The Property Right Paradigm, in: Journal of Economic History, 33, S. 16-27.

Allen, D. W. (2000): Transaction Costs, in: Encyclopedia of Law and Economics, 1, S. 893-926.

Allen, D. W. und Lueck, D. (1996): The Transaction Cost Approach to Agricultural Contracts, in: Martimort, D. (Hrsg.): Agricultural Markets: Mechanisms, Failures and Regulations. Amsterdam, S. 31-64.

Anderson, M. (1995): Conservation, the Environment, and the Farm Bill. Water Resources Update, in: Universities Council on Water Resources, 101, S. 4-11. Ariely, D., Ockenfels, A. und Roth, A. E. (2006): An Experimental Analysis of Ending Rules in Internet Auctions, zur Veröffentlichung angenommen in: The RAND Journal of Economics. Hier zitiert nach: http://ockenfels.unikoeln.de/pubs.php?w=all (28.06.2006).

Arrow, K. J. (1969): The Organisation of Economic Activity: Issues Pertinent to the Choice of Market versus Non-Market Allocation, in: The Analysis and Evaluation of Public Expenditures: The PBB-System. Joint Economic Committee, 91th Congress, 1st Session. Band 1. Washington, DC.

Arrow, K. J. (1970): Essays in the Theory of Risk-Bearing. Amsterdam.

Arrow, K. J. (1985): The Economics of Agency, in: Pratt, J. W. und Zeckhauer, R. J. (Hrsg.): Principals and Agents: The Structure of Business. Boston, S. 37-51.

Barnard, C. I. (1938): The Functions of the Executive. Cambridge.

Baudoux, P., Kazenwadel, G. und Doluschitz, R. (1997): Agrarumweltprogramme: Betriebliche Wirkungen und Einstellungen von Landwirten, in: Agrarwirtschaft, 4/5, S. 184-197. 
Baumgärtner, S. (2002): Der ökonomische Wert der biologischen Vielfalt, in: Bayerische Akademie für Naturschutz und Landschaftspflege (Hrsg.): Grundlagen zum Verständnis der Artenvielfalt und seiner Bedeutung und der Maßnahmen dem Artensterben entgegen $\mathrm{zu}$ wirken. Laufener Seminarbeiträge, 2, S. 73-90.

Beckmann, V. (1997): Transaktionskosten und institutionelle Wahl in der Landwirtschaft: zwischen Markt, Hierarchie und Kooperation. Diss. Göttingen.

Bertke, E. (2002): Erhaltung und Herstellung von Extensivgrünland - Honorierung in einem ergebnisorientierten Ansatz, in: Mitteilungen aus der NNA, 1, S. 6.

Bertke, E. (2005): Ökologische Güter in einem ergebnisorientierten Honorierungssystem für ökologische Leistungen der Landwirtschaft Herleitung, Definition, Kontrolle. Göttingen. Univ. Diss. Stuttgart.

Bertke, E., Isselstein, J. und Gerowitt, B. (2002): Ökologische Güter der pflanzlichen Biodiversität in einem Konzept zur ergebnisorientierten Honorierung ökologischer Leistungen der Landwirtschaft, in: Treffpunkt Biologische Vielfalt, II, S. 191-198.

Bertke, E., Gerowitt, B., Hespelt, S.-K., Isselstein, J., Marggraf, R. und Tute, C. (2003a): Ein regional verankertes Konzept zur ergebnisorientierten Honorierung ökologischer Leistungen der Landwirtschaft, in: Arbeitsgemeinschaft Grünland und Futterbau in der Gesellschaft für Pflanzenbauwissenschaften (Hrsg.): 47. Jahrestagung vom 28. bis 30. August 2003 in Braunschweig. Mitteilungen der Arbeitsgemeinschaft Grünland und Futterbau. Band 5. Göttingen, S. 243-246.

Bertke, E., Gerowitt, B. und Isselstein, J. (2003b): Regionale Grünlandtypen Erhaltung, Herstellung und Honorierung in einem ergebnisorientierten Ansatz, in: Mitteilungen der Biologischen Bundesanstalt für Land- und Forstwirtschaft, 393, S. 46-53.

Bertke, E., Hespelt, S.-K. und Tute, C. (2003c): Ergebnisorientierte Honorierung ökologischer Leistungen der Landwirtschaft. Jahrestagung der biologischen Stationen NRW in Wuppertal am 23. Oktober 2002 zum Thema Angebotsnaturschutz - Vorschläge zur Weiterentwicklung des Vertragsnaturschutzes, in: BfN-Scripten, 89, S. 27-39. 
Bertke, E., Groth, M. und Richter gen. Kemmermann, A. (2005): Die Honorierung von Umweltleistungen per Ausschreibung - Ergebnisse der modellhaften Umsetzung eines marktanalogen Agrarumweltprogramms, in: Ländlicher Raum, November/Dezember, S. 41-44.

Blackburn, W. H., Newman, J. B. und Wood, J. C. (1991): The Conservation Reserve Program: Effects on Soil, Water and Environmental Quality, in: Joyce, L. A., Mitchell, J. E. und Skold, D. M. (Hrsg.): The Conservation Reserve - Yesterday, Today and Tomorrow. Symposium Proceedings, January 14, 1991, Washington D. C. USDA Forest Service General Technical Report RM-203, S. 27-36.

BMVEL - Bundesministerium für Verbraucherschutz, Ernährung und Landwirtschaft (2004): Meilensteine der Agrarpolitik - Umsetzung der europäischen Agrarreform in Deutschland. Berlin.

Börner, C. S. und Macher, J. T. (2001): Transaction Cost Economics: An Assessment of Empirical Research in the Social Science. Arbeitspapier: http://faculty.msb.edu/jtm4/Papers/JLEO.pdf (23.05.2006).

Boyd, R. und Richerson, P. J. (2001): Norms and Bounded Rationality, in: Gigerenzer, G. und Selten, R. (Hrsg.): Bounded Rationality: The Adaptive Toolbox. Cambridge, S. 281-296.

Brehm, I. (2000): Das Conservation Reserve Program. Eine Möglichkeit zur ökonomisch und ökologisch effizienten Entlohnung von Umweltleistungen? Unveröffentlichte Diplomarbeit an der Fakultät für Landwirtschaft und Gartenbau Freising-Weihenstephan. München.

Brennan, G. und Buchanan, J. M. (1988): Besteuerung und Staatsgewalt. Analytische Grundlagen einer Finanzverfassung. Hamburg.

Brennan, G. und Buchanan, J. M. (1993): Die Begründung von Regeln. Konstitutionelle Politische Ökonomie. Tübingen.

Buchanan, J. M. (1991): Constitutional Economics. Oxford. Cambridge.

Buchanan, J. M. und Tullock, G. (1962): The Calculus of Consent. Logial Foundations of Constitutional Democracy. Michigan.

Bulow, J. und Roberts, J. (1989): The Simple Economics of Optimal Auctions, in: Journal of Political Economy, 5, S. 1060-1090.

Cansier, D. (1993): Umweltökonomie. Stuttgart. 
Cason, T. N. und Gangadharan, L. (2003): A Laboratory Comparision of Uniform and Discriminative Price Auctions for Reducing Non-Point Source Pollution. Research Paper Number 882. Department of Economics. The University of Melbourne.

Cason, T. N. und Gangadharan, L. (2004): Auction Design for Voluntary Conservation Programs, in: American Journal of Agricultural Economics, 86, S. 1211-1217.

Cezanne, W. und Mayer, A. (1998): Neue Institutionenökonomik - Ein Überblick, in: WISU, 11/98, S. 1345-1352.

Claasen, R., Hansen, L., Breneman, V., Weinberg, M., Cattaneo, A., Feather, P., Gadsby, D., Hellerstein, D., Hopkins, J., Johnston, P., Morehart, M. und Smith, M. (2001): Agri-Environmental Policy at the Crossroads: Guideposts on a Changing Landscape. Agricultural Economics Report No. 794. United States Department of Agricultural Economics. Economic Research Service.

Coase, R. H. (1937): The Nature of the Firm, in: Economia, IV, S. 386-405.

Coase, R. H. (1960): The Problem of Social Costs, in: Journal of Law and Economics, III, S. 1-44.

Coase, R. H. (1984): The New Institutional Economics, in: Journal of Institutional and Theoretical Economics, 140, S. 229-231.

Colby, B. G. (1990): Transaction Costs and Efficiency in Western Water Allocation, in: American Journal of Agricultural Economics, 12, S. 1184-1192.

Commons, J. R. (1931): Institutional Economics, in: American Economic Review, 21, S. $648-657$.

Commons, J. R. (1934): Institutional Economics. It’s Place in Political Economy. Madison, WI.

Cook, K. S. und Emerson, R. M. (1984): Exchange Networks and the Analysis of Complex Organisations, in: Bacharach, S. B. und Lawler, E. J. (Hrsg.): Research in the Sociology of Organizations. Greenwich. London, S. 1-30.

Curie, M. und Messori, M. (1998): New Institutional and New Keynesian Economics, in: Arena, R. und Longhi, C. (Hrsg.): Markets and Organisation. Berlin, S. 171-204.

Dahlmann, C. L. (1979): The Problem of Externality, in: Journal of Law and Economics, 22, S. 141-162. 
Das, S. R. und Sundaram, R. K. (1997): Auction Theory: A Summary with Applications to Treasury Markets. National Bureau of Economic Research, NBER-Working Paper Series. Working Paper 5873.

Demsetz, H. (1967): Toward a Theory of Property Rights, in: American Economic Review, 2, S. 347-359.

Demsetz, H. (1968): The Cost of Transacting, in: Quarterly Journal of Economics, 82, S. 33-53.

Demsetz, H. (1969): Information and Efficiency: Another Viewpoint, in: Journal of Law and Economics, 12, S. 1-22.

Der Ökosystemare Ansatz (2002): http://www.biodivchm.de/konvention/F1052472515/HTML_Page1053441544 (19.04.2006).

Dietl, H. (1991): Institutionen und Zeit. München.

Dow, G. K. (1987): The Function of Authority in Transaction Cost Economics, in: Journal of Economic Behaviour and Organization, 8, S. 13-38.

Downs, A. (1968): Ökonomische Theorie der Bürokratie. Tübingen.

Dugger, W. M. (1983): The Transaction Cost Analysis of Oliver E. Williamson. A New Synthesis?, in: Journal of Economic Issues, 1, S. 95-114.

Erhart, K. M. (2005): Auktionen. Vorlesungsskript des Instituts für Wirtschaftstheorie und Operations Research der Universität Karlsruhe.

Falconer, K. und Whitby, M. (1999): Administrative Costs in Agricultural Policies: The Case of the English Environmental Sensitive Areas. Research Report. University of Newcastle. Centre for Rural Economy.

Falconer, K., Dupraz, P. und Whitby, M. (2001): An Investigation of Policy Administrative Costs Using Panel Data for the English Environmental Sensitive Areas, in: Journal of Agricultural Economics, 52, S. 83-103.

Fama, E. (1980): Agency Problems and the Theory of the Firm, in: Journal of Political Economy, 88, S. 288-307.

Feather, P., Hellerstein, D. und Hansen, L. (1998): Exploring Methods of Selecting Cropland for Conservation, in: Agricultural Outlook, October, S. 21-24.

Feather, P., Hellerstein, D. und Hansen, L. (1999): Economic Valuation of Environmental Benefits and the Targeting of Conservation Programs: The Case of the CRP. Agricultural Economics Report No. 778. United States Department of Agricultural Economics. Economic Research Service. 
Feldmann, H. (1995): Eine institutionelle Revolution? Zur dogmenhistorischen Bedeutung der modernen Institutionenökonomik. Berlin.

Figgis, P. (2003): The Changing Face of Nature Conservation: Reflection on the Australian Experience, in: Adams, W. M. und Mulligan, M. (Hrsg.): Decolonizing Nature: Strategies for Conservation in a Postcolonial Era. London, S. 197-219.

Fischer, A., Hespelt, S.-K. und Marggraf, R. (2003): Ermittlung der Nachfrage nach ökologischen Gütern der Landwirtschaft - Das Northeim-Projekt, in: Agrarwirtschaft, 8, S. 390-393.

Fischer, M. (1994): Die Theorie der Transaktionskosten, in: Wirtschaftswissenschaftliches Studium, 11, S. 582-584.

Föhr, S. (1991): Ökonomische Analyse der internen Organisation. Wiesbaden.

Freeland, R. F. (1996): The Myth of the M-Form? Governance, Consent, and Organisational Change, in: American Journal of Sociology, 102, S. 481-526.

Frese, E. (1990): Organisationstheorie. Stand und Aussagen aus betriebswirtschaftlicher Sicht. Wiesbaden.

Furubotn, E. G. und Pejovich, S. (1972): Property Rights and Economic Theory: A Survey of Recent Literature, in: Journal of Economic Literature, 10, S. 11371162.

Furubotn, E. G. und Pejovich, S. (1974): The Economics of Property Rights. Cambridge, MA.

Furubotn, E. G. und Richter, R. (1991): The New Institutional Economics: An Assessment, in: Furubotn, E. G. und Richter, R. (Hrgs.): The New Institutional Economics. Texas, S. 1-32.

Garbe, C. (1994): Das Subsidiaritätsprinzip in der europäischen Union - Eine konzeptionelle Betrachtung. Göttingen.

Gerhard, T. (1995): Theorie und Realität ökonomischer Organisation. Der transaktionskostentheoretische Ansatz zur vertikalen Integration. Wiesbaden.

Gerowitt, B., Isselstein, J. und Marggraf, R. (2003): Rewards for Ecological Gods Requirements and Perspectives for Agriculture Land Use, in: Agriculture, Ecosystems and Environment. Biotic Indicators for Biodiversity and Sustainable Agriculture, 98, S. 541-547. 
Gerowitt, B. und Marggraf, R. (2001): Naturschutz als Produktionszweig für die (ökologische) Landwirtschaft - Neue Wege durch die ergebnisorientierte Honorierung, in: BfN-Skripten, 53, S. 18-23.

Granovetter, M. (1985): Economic Action and Social Structure: The Problem of Embeddedness, in: American Journal of Sociology, 91, S. 481-510.

Granovetter, M. (2002): A Theoretical Agenda for Economic Sociology, in: Guillen, M. F., Collins, R., England, P. und Meyer, M. (Hrsg.): Economic Sociology at the Millennium. New York.

Grossman, S. J. und Hart, O. D. (1983): An Analysis of the Principal-Agent Problems, in: Econometrica, 1, S. 7-45.

Hart, R. und Latacz-Lohmann, U. (2005): Combating Moral Hazard in AgriEnvironmental Schemes: A Multiple Agent Approach, in: European Review of Agricultural Economics, 1, S. 75-91.

Heissenhuber, A. und Lippert, C. (2000): Multifunktionalität und Wettbewerbsverzerrung, in: Agrarwirtschaft, 49, S. 249-252.

Hespelt, S.-K. (2002): Marktwirtschaftliche Einbindung eines ergebnisorientierten Honorierungssystems für ökologische Leistungen der Landwirtschaft, in: BfN-Scripten, Treffpunkt biologische Vielfalt, II, S. 199-204.

Hespelt, S.-K. (2005): Regionalisierung von Agrarumweltmaßnahmen - Dargestellt am Beispiel der transdisziplinären Entwicklung eines ergebnisorientierten, regional verankerten Honorierungssystems für ökologische Leistungen der Landwirtschaft unter besonderer Berücksichtigung eines regionalen Gremiums. Göttingen. Univ. Diss. Stuttgart.

Hespelt, S.-K. und Bertke, E. (2003): Ergebnisorientierte Honorierung ökologischer Leistungen der Landwirtschaft - Perspektiven für ein Agrarumweltprogramm der Zukunft, in: Nova Acta Leopoldina NF 87, 328, S. 343-346.

Hirshleifer, J. (1986): Economics from a Biological Viewpoint, in: Barney, J. B. und Ouchi, W. G. (Hrsg.): Organizational Economics. Toward a New Paradigm for Understanding and Studying Organizations. San Francisco. London, S. 319-371.

Hofmann, H., Rauh, R., Heißenhuber, A. und Berg, E. (1995): Umweltleistungen der Landwirtschaft. Konzepte zur Honorierung. Teubner-Reihe Umwelt. Stuttgart. Leipzig. 
Holm-Müller, K., Radke, V. und Weis, J. (2002): Umweltfördermaßnahmen in der Landwirtschaft - Teilnehmerauswahl durch Ausschreibungen?, in: Agrarwirtschaft, 2, S. 112-120.

Holm-Müller, K. und Hilden, R. (2005): Ausschreibung von Agrarumweltprogrammen am Beispiel der Grünlandextensivierung, in: Schriften der Gesellschaft für Wirtschafts- und Sozialwissenschaften des Landbaus e.V., 40, S. 215-223.

Holstrom, B. (1979): Moral Hazard and Observability, in: Bell Journal of Economics, 10, S. 74-91.

James, H. S. (2006): Annotated Bibliography on Transaction Cost Economics: http://www.ssu.missouri.edu/faculty/HJames/tce-bib.htm (12.04.2006).

Jehiel, P. und Moldovanu, B. (2003): An Economic Perspective on Auctions, in: Economic Policy, April, S. 271-308.

Jensen, M. C. und Meckling, W. H. (1976): Theory of the Firm: Managerial Behavior, Agency Costs and Ownership Structure, in: Journal of Financial Economics, 3, S. 305-360.

Kirchgässner, G. (1991): Homo oeconomicus. Das ökonomische Modell individuellen Verhaltens und seine Anwendung in den Wirtschafts- und Sozialwissenschaften. Tübingen.

Kitzner, I. M. (1973): Competition and Entrepreneurship. Chicago. London.

Klein, P. G. und Shelanski, H. (1995): Empirical Work in Transaction Cost Economics, in: Journal of Law and Economics, 11, S. 335-362.

Kleijn, D. und Sutherland, W. J. (2003): How effective are European Agrienvironmental Schemes in Conserving and Promoting Biodiversity?, in: Journal of Applied Ecology, 40, S. 947-969.

Klemperer, P. (1999): Auction Theory: A Guide to the Literature, in: Journal of Economic Surveys, 13, S. 227-286.

Klemperer, P. (2002): What really matters in Auction Design, in: Journal of Economic Perspectives, 16, S. 169-190.

Klemperer, P. (2004): Auctions: Theory and Practice. Online-Buch: http://www.paulklemperer.org (12.03.2006).

Knauer, N. (1993): Ökologie und Landwirtschaft. Situation - Konflikte - Lösungen. Stuttgart.

Knight, F. (1922): Risk, Uncertainty, and Profit. New York. 
KOM - Europäische Kommission (1998): Evaluation von AgrarUmweltprogrammen. Anwendungsstand der Verordnung (EWG) Nr. 2078/92. Arbeitsdokument der Kommission - GD VI (VI/7655/98).

Kräkel, M. (1992): Auktionstheorie und interne Organisation. Wiesbaden.

Krishna, V. (2002): Auction Theory. San Diego.

Latacz-Lohmann, U. (1993): Ausgestaltung des Prämiensystems als Mittel zur Steigerung der Effektivität von Extensivierungs- und Vertragsnaturschutzprogrammen, in: Agrarwirtschaft, 10, S. 351-358.

Latacz-Lohmann, U. (2004): Dealing with Limited Information in Designing and Evaluating Agri-environmental Policy. Präsentierter Beitrag im Rahmen des "90th European Seminar of the European Association of Agricultural Economics: Multifunctional agriculture, policies and markets: understanding the critial linkages“. Rennes. 28. und 29. Oktober 2004.

Latacz-Lohmann, U. und Van der Hamsvoort, C. (1997): Auctioning Conservation Contracts: A Theoretical Analysis and an Application, in: American Journal of Agricultural Economics, 79, S. 407-418.

Latacz-Lohmann, U. und Van der Hamsvoort, C. (1998): Auctions as a Means of Creating a Market for Public Goods from Agriculture, in: Journal of Agricultural Economics, 3, S. 334-345.

Latacz-Lohmann, U. und Hodge, I. (2003): European Agri-environmental Policy for the 21st Century, in: The Australian Journal of Agricultural and Resource Economics, 47, S. 123-139.

Latacz-Lohmann, U. und Schilizzi, S. (2005): Auctions for Conservation Contracts: A Review of the theoretical and empirical Literature - Report to the Scottish Executive Environment and Rural Affairs Department: http://www.scotland.gov.uk/Ressource/Doc/93853/0022574.pdf (02.05.2006). Laycock, W. A. (1991): The Conservation Reserve Program - How did we get where we are and where do we go from here?, in: Joyce, L. A., Mitchell, J. E. und Skold, D. M. (Hrsg.): The Conservation Reserve - Yesterday, Today and Tomorrow. Symposium Proceedings. January 14, 1991, Washington D. C. USDA Forest Service General Technical Report RM-203, S. 27-36.

Leblebici, H. (1985): Transaction Costs and Organisational Forms. A Re-Analysis, in: Organizational Studies, 6, S. 97-115. 
Lecheler, H. (1993): Das Subsidiaritätsprinzip - Strukturprinzip einer europäischen Union. Berlin.

Lee, D. R. und McKenzie, R. B. (1987): Regulating Government. A Preface to Constitutional Economics. Toronto.

Leitzinger, H. (1988): Submission und Preisbildung. Köln.

Llewellyn, K. N. (1931): What Price Contract? An Essay in Perspective, in: Yale Law Journal, 40, S. 704-751.

Löchel, H. (1995): Institutionen, Transaktionskosten und wirtschaftliche Entwicklung: ein Beitrag zur Institutionenökonomik und zur Theorie von Douglass C. North. Volkswirtschaftliche Schriften 444. Berlin.

Macken, J. (2002): Saving the Great Outdoors, in: Australian Financial Report, 6, S. 81.

McCann, L. und Easter, K. W. (1999): Transaction Costs of Policies to Reduce Agriculture Pollution in the Minnesota River, in: Land Economics, 8, S. 402414.

Macneil, I. R. (1974): The Many Futures of Contracts, in: Southern California Law Review, 47, S. 691-816.

Macneil, I. R. (1978): Contracts: Adjustment of Long-term Economic Relations under Classical, Neoclassical, and Relational Contract Law, in: Northwestern University Law Review, 72, S. 854-905.

Mann, S. (2001): Zur Effizienz der deutschen Agrarverwaltung, in: Agrarwirtschaft, 5, S. 302-307.

March, J. G. und Simon, H. A. (1958): Organisations. New York.

Marshall, A. (1890): Principles of Economics. 8. Aufl., London 1952.

Marggraf, R. (2003): Comparative Assessment of Agri-Environmental Programmes in Federal States of Germany, in: Agriculture, Ecosystems and Environment, 98, S. 507-516.

Marggraf, R., Bergmann, H., Bertke, E., Gerowitt, B. und Hespelt, S.-K. (2005): Effiziente und effektive Ausgestaltung der Agrarumweltprogramme Arbeitsgruppe 1: Thesen, in: Czybulka, D. (Hrsg.): Wege zu einem wirksamen Naturschutz: Erhaltung der Biodiversität als Querschnittsaufgabe. Beiträge zum Landwirtschaftsrecht und zur Biodiversität, Band III. BadenBaden, S. 93-102. 
McAfee, P. und McMillian, J. (1987): Auctions and Bidding, in: Journal of Economic Literature, 25, S. 699-738.

Mello, I., Heissenhuber, A. und Kantelhardt, J. (2002): Das Conservation Reserve Program der USA - Eine Möglichkeit zur effizienten Entlohnung von Umweltleistungen der Landwirtschaft?, in: Berichte über Landwirtschaft, 1, S. 85-93.

Menger, C. (1968): Grundsätze der Volkswirtschaftslehre. Gesammelte Werke. Band 1. Tübingen.

Milgrom, P. R. (1989): Auctions and Biding: A Primer, in: Journal of Economic Perspectives, 3, S. 3-22.

Mill, J. S. (1848): The Principles of Political Economy. London.

Moldovanu, B. (1996): William Vickrey und die Auktionstheorie - Anmerkungen zum Nobelpreis 1996, in: Wirtschaftsdienst, XII, S. 651-656.

Motyka, W. (1989): Erklärungspotential und praktische Eignung organisationstheoretischer Ansätze. München.

Musgrave, R. A., Musgrave, P. B. und Kullmer, L. (1994): Die öffentlichen Finanzen in Theorie und Praxis.

NAP - National Action Plan for Salinity and Water Quality (2003): A Guide to The National Marked-Based Instruments Pilots Program: http://www.napswq.gov.au/mbi/pubs/guide.pdf (14.04.2006).

NAP - National Action Plan for Salinity and Water Quality (2005): National Marketbased Instruments Pilots Program: http://www.napswq.gov.au/mbi (14.04.2006).

NECMA - North East Catchment Management Authority (2005): Bush Tender Trial: http://www.necma.vic.gov.au/programs/biodiversity/prior/bush_tender/ (16.05.2006).

Niedersächsisches Landesamt für Statistik (1999): Landwirtschaftszählung 1999, Landwirtschaftliche Betriebe und deren Flächen nach Hauptnutzungs- und Kulturarten. Hannover.

Niedersächsisches Landesamt für Statistik (2003): Statistische Berichte Niedersachsen. Bodennutzung und Ernte 2003. Hannover.

Nordhaus, W. D. (1975): The Political Business Cycle, in: Review of Economic Studies, 129, S. 169-190. 
North, D. C. (1988): Theorie des institutionellen Wandels. Eine neue Sicht der Wirtschaftsgeschichte. Tübingen.

North, D. C. (1990): Institutions, Institutional Change and Economic Performance. Cambridge.

North, D. C. und Thomas, R. P. (1973): The Rise of the Western World. A New Economic History. Cambridge.

North, D. C. und Wallis, J. J. (1994): Integrating Institutional Change and Technical Change in Economic History: A Transaction Cost Approach, in: Journal of Institutional and Theoretical Economics, 4, S. 609-624.

Ockenfels, A. und Roth, A. E. (2006): Late and Multiple Bidding in Second Price Internet Auctions: Theory and Evidence Concerning Different Rules for Ending an Auction, in: Games and Economic Behavior, 55, S. 297-320.

OECD - Organisation for Economic Co-Operation and Development (2001a): Multifunctionality - Towards an Analytical Framework. Paris.

OECD - Organisation for Economic Co-Operation and Development (2001b): Transaction Costs and Multifunctionality: Main Issues. Paris: http://www1oecd.org/agr/mf/doc/Transactioncosts32.pdf (12.04.2006).

OECD - Organisation for Economic Co-Operation and Development (2004): OECD Environmental Strategy - 2004 Review of Progress.

Osborn, T., Llacuna, F. und Linsenbigler, M. (1995): The Conservation Reserve Program - Enrollment Statistics for Signup Periods 1-12 and Fiscal Years 1986-93. Statistical Bulletin No. 925. United States Department of Agricultural Economics. Economic Research Service.

Osborn, T. (1997): Conservation Reserve Program, in: Anderson, M. und Magleby, R. (Hrsg.): Agricultural Resources and Indicators, 1996-97. Agricultural Handbook No. 712. United States Department of Agricultural Economics. Economic Research Service, S. 286-296.

Pejovich, S. (1971): Toward a General Theory of Property Rights, in: Zeitschrift für Nationalökonomie, 1/2, S. 141-155.

Pejovich, S. (1972): Toward an Economic Theory of the Creation and Specification of Property Rights, in: Review of Social Economy, 3, S. 309-325.

Perrow, C. (1982): Markets, Hierarchies and Hegemony, in: Van de Ven, A. H. und Joyce, W. F. (Hrsg.): Perspectives on Organizations Design and Behavior. New York, S. 371-386. 
Perrow, C. (1986): Complex Organisations. Critical Essay. New York.

Pfaff, D. und Zweifel, P. (1998): Die Principal-Agent Theorie - Ein fruchtbarer Beitrag der Wirtschaftstheorie zur Praxis, in: WiSt - Zeitschrift für Ausbildung und Hochschulkontakt, April, S. 184-190.

Picot, A. (1982): Transaktionskostenansatz in der Organisationstheorie. Stand der Dinge und Aussagewert, in: Die Betriebswirtschaftslehre, 2, S. 276-284.

Picot, A. und Dietl, H. (1990): Transaktionskostentheorie, in: Wirtschaftswissenschaftliches Studium, 19, S. 178-184.

Picot, A. und Dietl, H. (1993): Neue Institutionenökonomik und Recht, in: Ott, C. und Schäfer, H. B. (Hrsg.): Ökonomische Analyse des Unternehmensrechts. Heidelberg, S. 307-330.

Pies, I. (2001): Theoretische Grundlagen demokratischer Wirtschafts- und Gesellschaftspolitik - Der Beitrag Oliver Williamsons, in: Pies, I. und Leschke, M. (Hrsg.): Oliver Williamsons Organisationsökonomik. Tübingen, S. 1-27.

Plankl, R. (1998): Die Festsetzung von Prämien im Rahmen der Förderung einer umweltverträglichen Agrarproduktion - „Bookbuilding“ als modifiziertes Ausschreibungsverfahren, in: Landbauforschung Völkenrode, 1, S. 44-51.

Plankl, R. (1999): Honorierung ökologischer Leistungen - Erfahrungen mit dem USamerikanischen „Conservation Reserve Program“ (CRP). Landbauforschung Völkenrode, Sonderheft 201, S. 163-175.

Pommerehne, W. W. und Römer, A. U. (1992): Ansätze zur Erfassung der Präferenzen für öffentliche Güter, in: Jahrbuch für Sozialwissenschaft, 43, S. 171-210.

Powell, W. W. (1990): Neither Market nor Hierarchy: Network Forms of Organizations, in: Staw, B. M. und Cummings, L. L. (Hrsg.): Research in Organizational Behaviour. Greenwich, S. 295-336.

Pratt, J. W. und Zeckhauser, R. J. (1985): Principals and Agents. The Structure of Business. Boston.

Reichelderfer, K. und Boggess, W. G. (1988): Government Decision Making and Program Performances: The Case of the Conservation Reserve Program, in: American Journal of Agricultural Economics, 1, S. 1-10. 
Ribaudo, M. O., Hoag, D. L., Smith, M. E. und Heimlich, R. (2001): Environmental Indices and the Politics of the Conservation Reserve Program, in: Ecological Indicators, 1, S. 11-20.

Richter, R. (1991): Institutionenökonomische Aspekte der Theorie der Unternehmung, in: Ordelheide, D., Rudolph, B. und Büsselmann, E. (Hrsg.): Betriebswirtschaftslehre und Ökonomische Theorie, S. 395-429.

Richter, R. (1994): Institutionen ökonomisch analysiert. Zur jüngeren Entwicklung auf einem Gebiet der Wirtschaftstheorie. Tübingen.

Richter, R. (2001): New Economic Sociology and New Institutional Economics. Paper presented at the Annual Conference of the International Society for New Institutional Economics (ISNIE), Manuskript: http://www.unisaarland.de/fak1/fr12/albert/mitarbeiter/richter/institut/revise4.pdf (11.05.2006).

Richter, R. (2001): Oliver Williamsons Organisationsökonomik, in: Pies, I. und Leschke, M. (2001): Oliver Williamsons Organisationsökonomik, S. 225-237. Richter, R. und Furubotn, E. G. (2003): Neue Institutionenökonomik. Tübingen.

Riekhof, H.-C. (1984): Unternehmensverfassungen und Theorie der Verfügungsrechte. Wiesbaden.

Römhild, W. (1997): Preisstrategien bei Ausschreibungen. Berlin.

Rüffer, C. (in Vorbereitung): Merit goods determined by society value judgments Political implications for public participation. Results from an empirical analysis concerning environmental goods.

Samuelson, P. A. (1954): The Pure Theory of Public Expenditure, in: Review of Economics and Statistics, 36, S. 387-389.

Schanz, G. (1988): Erkennen und Gestalten. Betriebswirtschaftslehre in kritischrationaler Absicht. Stuttgart.

Schedler, K. (1993): Anreizsysteme in der öffentlichen Verwaltung. Diss. Bern.

Schedler, K. und Proeller, I. (2000): New Public Management. Bern.

Schneider, D. (1985): Die Unhaltbarkeit des Transaktionskostenansatzes für die „Markt oder Unternehmung“ - Diskussion, in: Zeitschrift für Betriebswirtschaft, 55, S. 1237-1254.

Schneider, D. (1995): Betriebswirtschaftslehre. Band I: Grundlagen. Wien. München.

Schumpeter, J. A. (1946): Kapitalismus, Sozialismus und Demokratie. Bern. 
Shoemaker, R. (1989): Agricultural Land Values and Rents under the Conservation Reserve Program, in: Land Economics, 5, S. 131-137.

Shubik, M. (1983): Auctions, Bidding, and Markets: A Historical Sketch, in: Engelbrecht-Wiggans, M., Shubik, M. und Strak, J. (Hrsg.): Auctions, Bidding and Contracting. New York, S. 165-191.

Siebert, H. (1978): Ökonomische Theorie der Umwelt. Tübingen.

Simon, H. A. (1957): Models of Man: Social and Rational. Mathematical Essays on Rational Human Behavior in a Social Setting. New York. London. Sydney.

Simon, H. A. (1961): Administrative Behaviour. A Study of Decision-Making Processes in Administrative Organization. New York. London.

Simon, H. A. (1972): Theories of Bounded Rationality, in: McGuire, C. B. und Radner, R. (Hrsg.): Decisions and Organization. Amsterdam. London, S. 161176.

Smith, A. (1776/1904): An Inquiry into the Nature and Causes of the Wealth of Nations. Edwin Cannan’s annotated Edition. 1904.

Smith, M. E. (2003): Land Retirement, in: Heimlich, R. (Hrsg.): Agricultural Resources and Environmental Indicators. Agricultural Handbook No. 772, United States Department of Agricultural Economics. Economic Research Service.

Smith, R. B. W und Tomasl, T. D. (1995): Transaction Costs and Agricultural Nonpoint-Source Water Pollution Control Policies, in: Journal of Agricultural and Resource Economics, 20, S. 277-290.

SRU - Sachverständigenrat für Umweltfragen (1996): Konzepte einer dauerhaften umweltgerechten Nutzung ländlicher Räume. Sondergutachten. Stuttgart.

SRU - Sachverständigenrat für Umweltfragen (2002): Für eine Stärkung und Neuorientierung des Naturschutzes. Sondergutachten. Stuttgart.

Stigler, G. J. (1961): The Economics of Information, in: Journal of Political Economy, 69, S. 213-225.

Stoneham, G., Chaudhri, V., Ha, A. und Strappazzon, L. (2003): Auctions for Conservation Contracts: An empirical Examination of Victoria's BushTender Trial, in: Australian Journal of Agricultural and Resource Economics, 4, S. 477-500. 
Streit, M. E. und Wohlgemuth, M. (1999): Walter Eucken und Friedrich A. von Hayek - Initiatoren der Ordnungsökonomik. Diskussionsbeitrag 11-99. MaxPlank-Institut zur Erforschung von Wirtschaftssystemen. Jena.

Streissler, E. (1993): Das Problem der Internalisierung, in: König, H. (Hrsg.): Umweltverträgliches Wirtschaften als Problem von Wissenschaft und Politik. Schriften des Vereins für Socialpolitik. N.F. Berlin, S. 87-110.

Szentandrasi, S., Polasky, S., Berrens, R. und Leonard, J. (1995): Conserving Biological Diversity and the Conservation Reserve Program, in: Growth and Change, 26, S. 383-404.

Thiele, M. (1994): Neue Institutionenökonomik, in: Das Wirtschaftsstudium, 12, S. 993-997.

Tietzel, M. (1981): Die Ökonomie der Property Rights: Ein Überblick, in: Zeitschrift für Wirtschaftspolitik, 3, S. 207-243.

USDA - United States Department of Agriculture (2002a): Farmbill 2002.

USDA - United States Department of Agriculture (2002b): Farm Policy: Title II Conservation.

USDA - United States Department of Agriculture (2003a): Fact Sheet: Conservation Reserve Program.

USDA - United States Department of Agriculture (2003b): 2002 Farm Bill Conservation Reserve Program - Long-term Policy; Interim Rule. 7 CFR Part 1410. Federal Register 68 (89), S. 24829-24845.

USDA - United States Department of Agriculture (2003c): Fact Sheet: Conservation Reserve Program Sign-up 26, May 5 - June 13, 2003.

USDA - United States Department of Agriculture (2003d): Fact Sheet: Conservation Reserve Program Sign-up 26, Environmental Benefits Index.

USGAO - United States General Accounting Office (1993): Conservation Reserve

Program: Cost effectiveness is uncertain. United States General Accounting Office GAO /RCED-93-132. Washington D.C.

Vatn, A. (2001): Transaction Costs and Multifunctionality. Beitrag präsentiert im Rahmen des OECD Workshops "Mulitifunctionality: Applying the OECD Analytical Framework Guiding Policy Design” am 02.-03. Juli 2001 in Paris.

Vatn, A. (2002): Multifunctional Agriculture: Some Consequences for International Trade Regimes, in: European Review of Agricultural Economics, 3, S. 309327. 
Vesterby, M. (2003): Land Use, in: Heimlich, R. (Hrsg.): Agricultural Resources and Environmental Indicators. Agricultural Handbook No. 772, United States Department of Agricultural Economics. Economic Research Service.

Vickrey, W. (1961): Counterspeculation, Auctions, and Competitive Sealed Tenders, in: The Journal of Finance, 16, S. 8-37.

Von Hayek, F. A. (1935a): Sozialistische Wirtschaftrechnung I: Natur und Geschichte des Problems, in: Von Hayek, F. A. (Hrsg.): Individualismus und wirtschaftliche Ordnung. Salzburg, S. 156-191.

Von Hayek, F. A. (1935b): Sozialistische Wirtschaftrechnung II: Der Stand der Diskussion, in: Von Hayek, F. A. (Hrsg.): Individualismus und wirtschaftliche Ordnung. Salzburg, S. 192-232.

Von Hayek, F. A. (1945): The Use of Knowledge in Society, in: American Economic Review, 35, S. 519-530.

Von Hayek, F. A. (1967): Studies in Philosophy, Politics and Economics. London.

Von Mises, L. (1922): Die Gemeinwirtschaft. Untersuchungen über den Sozialismus. Jena.

Voigt, S. (2002): Institutionenökonomik. München.

Wallis, J. J. und North, D. C. (1988): Measuring the Transaction Sector in the American Economy, 1870-1970, in: Engerman, S. L. und Gallman, R. E. (Hrsg.): Long-term Factors in American Economic Growth. Studies in Income and Wealth, S. 95-161.

Wang, N. (2003): Measuring Transaction Costs: An Incomplete Survey. Ronald Coase Institute Working Papers, Number 2, Februar 2003: http://www.coase.org/workingpapers/wp-2.pdf (23.02.2006).

Warnemünde, B. (1995): Aufgaben und Organisation der Agrarverwaltung auf der Ebene der Bundesländer - Eine Untersuchung aus ökonomischer Sicht. Göttingen.

Weimann, J. (1996): Wirtschaftspolitik - Allokation und kollektive Entscheidung. Berlin.

Weinert, A. B. (1998): Organisationspsychologie. Ein Lehrbuch. Weinheim.

Wicke, L. (1993): Umweltökonomie - Eine praxisorientierte Einführung. Berlin.

Wiggins, S. N. (1991): The Economics of the Firm and Contracts: A Selective Survey, in: Journal of Institutional and Theoretical Economics, 4, S. 603-661. 
Wilhelm, J. (1999): Ökologische und ökonomische Bewertung von Agrarumweltprogrammen: Delphi-Studie, Kosten-Wirksamkeits-Analyse und Kosten-Nutzen-Betrachtung. Göttingen, Univ., Diss. Frankfurt am Main. Berlin.

Williamson, O. E. (1971): The Vertical Integration of Production: Market Failure Considerations, in: American Economic Review, 61, S. 112-123.

Williamson, O. E. (1973): Organizational Forms and Internal Efficiency. Markets and Hierarchies. Some elementary considerations, in: American Economic Review, 63, S. 316-325.

Williamson, O. E. (1975): Markets and Hierarchies. Analysis and Antitrust Implications. New York.

Williamson, O. E. (1979): Transaction-cost Economics: The Governance of Contractual Relations, in: Journal of Law and Economics, 22, S. 233-261.

Williamson, O. E. (1985): The Economic Institutions of Capitalism. New York.

Williamson, O. E. (1990a): Die ökonomischen Institutionen des Kapitalismus. Tübingen.

Williamson, O. E. (Hrsg. 1990b): Organisation Theory: From Chester Barnard to the Present and Beyond. New York. Oxford.

Williamson, O. E. (1993): Transaction Cost Economics and Organization Theory, in: Industrial and Corporate Change, 2, S. 107-156.

Williamson, O. E. (1996): The Mechanisms of Governance. Oxford.

Williamson, O. E. (1998): The Institutions of Governance, in: American Economic Review, 88, S. 75-79.

Williamson, O. E. (2000): The New Institutional Economics: Taking Stocks, Looking Ahead, in: Journal of Economic Literature, 38, S. 595-613.

Williamson, O. E. und Masten, S. E. (1999): The Economics of Transaction Costs. Cheltenham.

Wolfstetter, E. (1996): Auctions: An Introduction; in: Journal of Economic Surveys, 10, S. 367-420.

Wolfstetter, E. (1998): Auktionen und Ausschreibungen - Bedeutung und Grenzen des „linkage“-Prinzips, in: Tietzel, M. (Hrsg.): Ökonomische Theorie der Rationierung. München, S. 139-161.

Wood, P. M. (2000): Biodiversity and Democracy - Rethinking Society and Nature. Vancouver. 
Yamin, M. (1996): Understanding Strategic Alliances. The Limits of Transaction Cost Economics, in: Coombs, R. et al. (Hrsg.): Technological Collaborations. The Dynamics in Industrial Innovation. Cheltenham, S. 165-179. 


\section{Verzeichnis der Gesetzesgrundlagen}

Biodiversitätskonvention (CBD) - Convention on Biological Diversity: http://www.biodiv.org/convention/convention.shtml (26.07.2006).

Haushaltsgrundsätzegesetz (HGrG) - Gesetz über die Grundsätze des Haushaltsrechts des Bundes und der Länder vom 19. August 1969, zuletzt geändert durch das Gesetz zur Änderung der Rechtsgrundlagen für die Vergabe öffentlicher Aufträge (VgRÄG) vom 26. August 1998, BGBl. I S. 2512.

Verdingungsverordnung für Bauleistungen (VOB) in der Bekanntmachung vom 12. September 2002, Bundesanzeiger. Nr. 202a vom 29. Oktober 2002.

Verordnung (EG) Nr. 1257/1999 des Rates vom 17. Mai 1999 über die Förderung der Entwicklung des ländlichen Raums durch den Europäischen Ausrichtungs- und Garantiefonds für die Landwirtschaft (EAGFL) und zur Änderung bzw. Aufhebung bestimmter Verordnungen.

Verordnung (EG) Nr. 1750/1999 der Kommission vom 23. Juli 1999 mit Durchführungsvorschriften zur Verordnung (EG) Nr. 1257/1999 des Rates über die Förderung und Entwicklung des ländlichen Raums durch den Europäischen Ausrichtungs- und Garantiefonds für die Landwirtschaft (EAGFL).

Verordnung (EG) Nr. 2419/2001 der Kommission vom Dezember 2001 mit Durchführungsbestimmungen zum mit der Verordnung (EWG) Nr. 3508/92 des Rates vom 27. November 1992 eingeführten Integrierten Verwaltungsund Kontrollsystems für bestimmte gemeinschaftliche Beihilferegelungen.

Verordnung (EG) Nr. 1782/2003 des Rates vom 29. September 2003 mit gemeinsamen Regeln für Direktzahlungen im Rahmen der Gemeinsamen Agrarpolitik und mit bestimmten Stützungsregelungen für Inhaber landwirtschaftlicher Betriebe.

Verordnung (EG) Nr. 1698/2005 des Rates vom 20. September 2005 über die Förderung der Entwicklung des ländlichen Raums durch den Europäischen Landwirtschaftsfonds für die Entwicklung des ländlichen Raums (ELER).

Verordnung (EWG) Nr. 2078/92 des Rates vom 30. Juni 1992 für umweltgerechte und den natürlichen Lebensraum schützende landwirtschaftliche Produktionsverfahren. 
Vertrag zur Gründung der Europäischen Gemeinschaft (2001) in der durch den Vertrag von Nizza geänderten Version vom 21. Februar 2002. Bundesgesetzblatt 2001, Teil II, S. 1667-1671. 


\section{Anhang}

Der Anhang befindet sich auf der beiliegenden CD-ROM und umfasst die Ausschreibungsunterlagen der Ausschreibungen 2004/2005 und 2006 sowie die Anschreiben und Fragebögen der drei im Rahmen der Arbeit durchgeführten schriftlichen Befragungen von Landwirten. Der Anhang ist in die folgenden fünf Ordner gegliedert, wobei jeder Ordner die entsprechenden Dokumente im PDFFormat beinhaltet.

Anhang 1: Die Ausschreibung 2004-2005

Anhang 2: Die Ausschreibung 2006

Anhang 3: Die erste Befragung von Landwirten (Sommer 2004)

Anhang 4: Die zweite Befragung von Landwirten (Herbst-Winter 2004)

Anhang 5: Die dritte Befragung von Landwirten (Frühjahr 2006) 